\title{
Laminar fMRI at ultra-high fields
}

Citation for published version (APA):

Kashyap, S. S. (2019). Laminar fMRI at ultra-high fields: Acquisition and analysis strategies. [Doctoral Thesis, Maastricht University]. Ipskamp Printing BV. https://doi.org/10.26481/dis.20190829sk

Document status and date:

Published: 01/01/2019

DOI:

10.26481/dis.20190829sk

Document Version:

Publisher's PDF, also known as Version of record

\section{Please check the document version of this publication:}

- A submitted manuscript is the version of the article upon submission and before peer-review. There can be important differences between the submitted version and the official published version of record.

People interested in the research are advised to contact the author for the final version of the publication, or visit the DOI to the publisher's website.

- The final author version and the galley proof are versions of the publication after peer review.

- The final published version features the final layout of the paper including the volume, issue and page numbers.

Link to publication

\footnotetext{
General rights rights.

- You may freely distribute the URL identifying the publication in the public portal. please follow below link for the End User Agreement:

www.umlib.nl/taverne-license

Take down policy

If you believe that this document breaches copyright please contact us at:

repository@maastrichtuniversity.nl

providing details and we will investigate your claim.
}

Copyright and moral rights for the publications made accessible in the public portal are retained by the authors and/or other copyright owners and it is a condition of accessing publications that users recognise and abide by the legal requirements associated with these

- Users may download and print one copy of any publication from the public portal for the purpose of private study or research.

- You may not further distribute the material or use it for any profit-making activity or commercial gain

If the publication is distributed under the terms of Article $25 \mathrm{fa}$ of the Dutch Copyright Act, indicated by the "Taverne" license above, 


\section{Laminar fMRI at ultra-high fields Acquisition and analysis strategies}
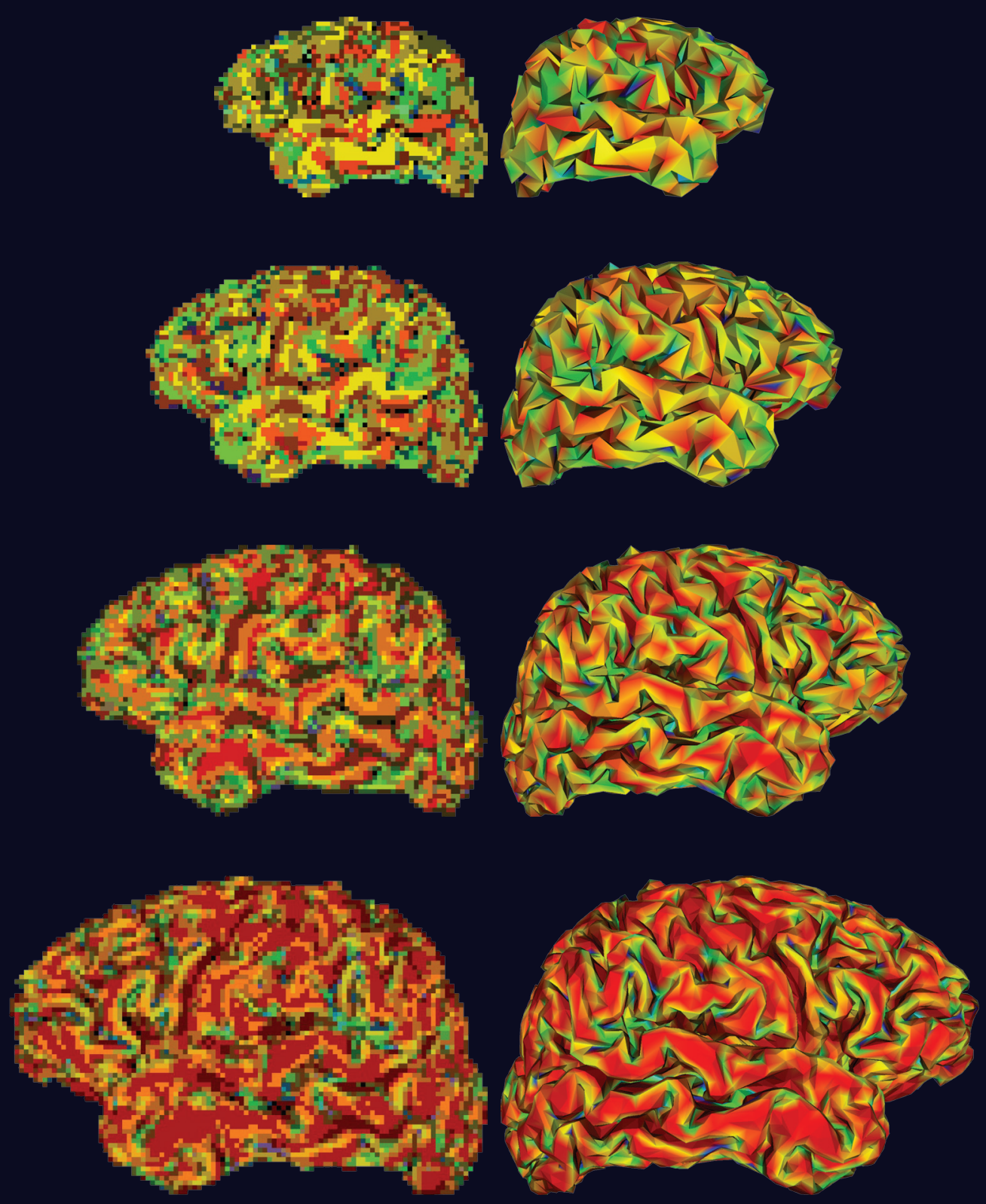

\section{Sriranga Kashyap}




\title{
Propositions accompanying the $\mathrm{PhD}$ thesis \\ Laminar fMRI at ultra-high fields Acquisition and analysis strategies
}

\author{
Sriranga Kashyap, 29th August 2019
}

1. High-resolution fMRI data must be analysed in native-EPI space to avoid loss of the acquired signal fidelity due to blurring via distortion-correction algorithms and multiple resampling steps.

2. Analyses in native-EPI space can be facilitated using distortion-matched anatomical data acquired with sequences, such as MI-EPI. The $\mathrm{T}_{1}$ estimates using MIEPI are comparable to Sa2RAGE-corrected MP2RAGE $\mathrm{T}_{1}$.

3. Different temporal segments of the BOLD signal time-course have different depth-depen-dence. Particularly, the post-stimulus undershoot is better localised to grey matter relative to the positive BOLD signal.

4. Anisotropic voxels allow sampling cortical layers or columns at unprecedented spatial scales (e.g. $0.1 \mathrm{~mm}$ ). At ultra-high resolutions, significantly better image SNR can be achieved using region-of-interest specific (e.g. occipital lobe) RF coils than with standard whole-brain bird-cage coils.

5. Higher spatial resolutions are required to reduce the 'blooming effect' of the GEBOLD signal at the pial surface. However, the ascending vein bias can only be addressed using modelling approaches.

6. Sub-millimetre resolution CBF fMRI is feasible at $7 \mathrm{~T}$ using arterial spin labelling with 3D-EPI readout. The simultaneously acquired laminar BOLD and CBF signals have contrasting depth-dependent behaviour that can be reconciled using established modelling approaches.

7. The principal inversion contrasts from the MP2RAGE sequence carry valuable tissue-specific information that can enable segmentation of non-brain tissues, such as skull, dura, vessels. Manual correction of anatomical segmentations is not just inevitable but essential in high-resolution studies.

8. Mesoscale (laminar/columnar) fMRI, through effective acquisition and data analysis, is the keystone that connects the micro- and macroscales and turn the tide for fMRI as a clinical tool.

9. Working with partial coverage fMRI data requires multi-tool integrated workflows with parameters optimised on a dataset-by-dataset basis.

10. There is no substitute for high resolution.

11. "The measure of an education is that you acquire some idea of the extent of your ignorance."

- Christopher Hitchens 


\section{Laminar fMRI at ultra-high fields}

Acquisition and analysis strategies

\section{Dissertation}

To obtain the degree of Doctor at Maastricht University, on the authority of the Rector Magnificus, Prof. Dr. Rianne M. Letschert, in accordance with the decision of the Board of Deans, to be defended in public

on Thursday, 29th of August 2019, at 1200 hours

by

Sriranga Subbakrishna Kashyap 


\section{Promotor}

Prof. Dr. Rainer Goebel

\section{Co-promotor}

Prof. Dr. Kâmil Uludağ

\section{Assessment Committee}

Prof. Dr. Peter de Weerd (Chair)

Prof. Dr. Elia Formisano

Dr. Laurentius Huber

Prof. Dr. Nikolaus Weiskopf

Max Planck Institute for Human Cognitive and Brain Sciences, Leipzig, Germany

Dr. Wietske van der Zwaag

Spinoza Centre for Neuroimaging, Amsterdam, The Netherlands

(C) Sriranga Kashyap, Maastricht 2019.

All rights reserved. No part of this publication may be reproduced, stored in a retrieval system or transmitted in any form or by any means, electronic, mechanical, photocopying, recording or otherwise, without prior written permission of the author.

Cover Sriranga Kashyap, 2019

Production Ipskamp Printing, The Netherlands

ISBN 978-94-028-1662-4 
To my dad . . . 



\section{Contents}

1 Introduction $\quad 1$

1.1 Nuclear Magnetic Resonance (NMR) . . . . . . . . . . . . . . . 3

1.2 Magnetic Properties of Haemoglobin . . . . . . . . . . . . 6

1.3 Blood oxygenation and functional MRI . . . . . . . . . . 7

1.4 Physiological bases of functional MRI . . . . . . . . . . . . 9

1.5 BOLD fMRI at ultra-high field . . . . . . . . . . . . . . . . 12

1.6 Cortical layers and laminar fMRI . . . . . . . . . . . . 13

1.7 Structure of the thesis . . . . . . . . . . . . . . 25

2 Impact of acquisition and analysis strategies $\quad \mathbf{2 9}$

2.1 Introduction . . . . . . . . . . . . . . . . . 33

2.2 Methods . . . . . . . . . . . . . . . . . . 38

2.3 Results . . . . . . . . . . . . . . . . 54

2.4 Discussion . . . . . . . . . . . . . . . . 62

2.5 Limitations and future considerations . . . . . . . . . . . 70

2.6 Concluding remarks . . . . . . . . . . . . . . . 74

3 Ultra-high resolution fMRI using anisotropic voxels $\quad 77$

3.1 Introduction . . . . . . . . . . . . . . 81

3.2 Methods . . . . . . . . . . . . . . . 85

3.3 Results . . . . . . . . . . . . . . . . 94

3.4 Discussion . . . . . . . . . . . . . . . . . . . 104

3.5 Limitations . . . . . . . . . . . . . . . . . . . . . 110

4 Laminar fMRI using arterial spin labelling $\quad 117$

4.1 Introduction . . . . . . . . . . . . . . . . . . . 121

4.2 Methods . . . . . . . . . . . . . . . . . . . . 124

4.3 Results . . . . . . . . . . . . . . . . . . 129

4.4 Discussion . . . . . . . . . . . . . . . . . 135

4.5 Limitations . . . . . . . . . . . . . . . . . . . 139 
5 General Discussion $\quad 143$

5.1 Data acquisition and processing . . . . . . . . . . . 145

5.2 Laminar analysis . . . . . . . . . . . . . . . . . . . . . 153

5.3 Vascular bias in GE-BOLD . . . . . . . . . . . . . . . . . 155

5.4 Non-BOLD alternatives . . . . . . . . . . . . . . 156

5.5 Concluding remarks and outlook . . . . . . . . . . 157

$\begin{array}{ll}\text { Bibliography } & 161\end{array}$

$\begin{array}{lr}\text { Valorisation } & 187\end{array}$

$\begin{array}{lc}\text { Research output } & 193\end{array}$

$\begin{array}{lr}\text { Acknowledgements } & 197\end{array}$

$\begin{array}{ll}\text { Curriculum Vitae } & 203\end{array}$ 




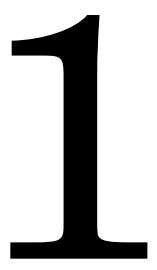

Introduction 

In his letter to Robert Hooke, Isaac Newton famously remarked, "If I have seen further, it is by standing on the shoulders of giants". In the $17^{\text {th }}$ century, this statement was a radical notion because most scholars at the time (Hooke included) considered the incremental, combinatorial nature of knowledge an affront to originality (or novelty), whereas Newton recognised that building upon earlier ideas was a natural function of how science progresses. Four centuries since, it is this spirit of scientific progress recognised by Newton that has resulted in several discoveries and inventions that have had "the greatest benefit to mankind" [1], none more so in the field of diagnostic healthcare and research than magnetic resonance imaging (MRI). Today, MRI is a highly multi- and inter-disciplinary field of research and innovation driven by the principles of physics on the foundation of rigorous mathematics, exploiting the behaviour of molecules in magnetic fields and engineered by cutting-edge technology to non-invasively visualise the inner structure and function of biological systems. Therefore, no introduction to the field would be complete without a brief discussion of the discoveries that led to MRI, the earliest of which can be traced back to the Institute of Physical Chemistry at the University of Hamburg.

\subsection{Nuclear Magnetic Resonance (NMR)}

In the 1930s, Otto Stern, a meticulous experimental physicist, developed the molecular beam method, which made it possible to observe a variety of physical properties of an isolated molecule and discovered the magnetic moment of protons [24] by magnetically deflecting a beam of molecular hydrogen. Soon after, physicist Isidor Rabi [5-7] building upon Stern's work, combined high-precision spectroscopy with the molecular ray technique. Using his improved molecular beam method, Rabi drove a stream of molecular hydrogen through a homogeneous mag- 
netic field and subjected it to radio frequency (RF) energy. This RF energy was absorbed by the molecules at a sharply defined frequency and caused a tiny measurable deflection of the beam. This was the first observation of the magnetic resonance phenomenon. However, Rabi's approach was limited to studies involving small molecules in a high vacuum. In 1946, Felix Bloch [8] and Edward Purcell [9] independently and near simultaneously demonstrated the NMR phenomenon at room temperature in liquids (water) and solids (paraffin) respectively. This discovery was followed by the foundational work for NMR imaging with the description of the basic principles of magnetic resonance [10] and development of pulsed NMR techniques [11, 12]. In 1973, Paul Lauterbur [13] demonstrated that by imposing magnetic field gradients across a sample, it was possible to define the spatial distribution of protons in water by different frequencies and a $2 \mathrm{D}$ image of the sample can be reconstructed by measuring with gradients along different directions. Around the same time, Sir Peter Mansfield [14, 15] developed the echo-planar imaging (EPI) technique that provided an alternative method of obtaining NMR images in a fraction of the time that was previously needed (order of milliseconds rather than minutes). Due to the sheer speed and robustness of EPI, artefacts due to movement could be reduced and allowed the possibility to improve the spatial resolution of the images. This set the wheels in motion for in vivo applications for NMR imaging because biological tissues contain different amounts of water and differences in relaxation rates can be distinguished using purpose-driven NMR pulse sequences and rapid acquisition of images ${ }^{1}$. Commercialisation of NMR imaging, now called Magnetic Resonance Imaging (MRI) [16], began in the 1980s for diagnostic use and the technique was rapidly adopted by

\footnotetext{
1 A thorough introduction to the fundamental MR principles, image reconstruction, contrast mechanisms, hardware and parallel imaging techniques can be found in in Magnetic Resonance Imaging: Physical Principles and Sequence Design by Brown, Haacke, Cheng, Thompson, and Venkatesan [10].
} 
clinicians due to the absence of ionising radiation, the variety of tissue-specific contrasts, higher detail and better quality structural images compared to existing modalities such as X-rays [17] or Computed Tomography (CT) [18].

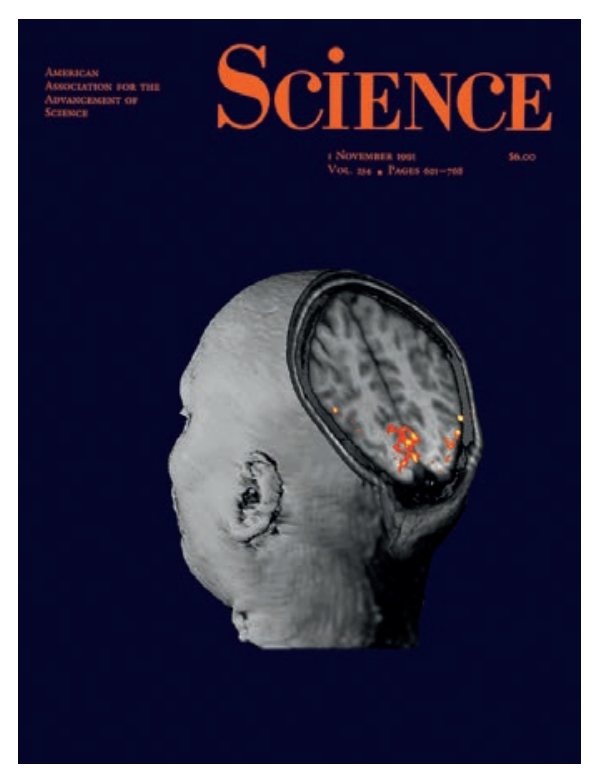

Figure 1.1: Image showing visual cortex activation from Belliveau and collegues [19] featured on the cover of the November 1991 issue of Science Magazine.
In the meantime, interest in functional neuroimaging had been picking up pace with studies using Positron Emission Tomography (PET) [20, 21] to measure stimulus-induced changes in Cerebral Blood Flow (CBF) [22], Cerebral Metabolic Rate of Oxygen $\left(\mathrm{CMRO}_{2}\right)$ [22] and Cerebral Blood Volume (CBV) [23] in humans. These early PET studies captured the imagination of MRI researchers who were using injections of Gadolinium-based contrast agents $[24,25]$ to map CBF and $\mathrm{CBV}$ changes in the brain. In 1991, Dynamic Susceptibility Contrast (DSC) enhanced MRI (DSC-MRI)

was applied for the first time to map functional CBV changes in response to visual stimulation in humans [19] and the study even made the cover of Science Magazine (Fig. 1.1). This was a turning point in the history of neuroimaging wherein it was demonstrated that anatomical structure and physiological changes related to brain function can be acquired in combination using a single imaging modality. While this was novel and lit up a whole new avenue for research, a major limita- 
tion of this approach for cognitive neuroscience applications was the administration of intravascular Gd-based contrast agents. The answer to this problem would emerge from the field of biophysics with the curious behaviour of haemoglobin in a magnetic field.

\subsection{Magnetic Properties of Haemoglobin}

Haemoglobin $(\mathrm{Hb})$ is multi-subunit globular protein complex with four globular protein subunits (tetramer) and each of containing a haem group (iron (Fe) ion centrally bound in a porphyrin ring) [26] present in erythrocytes (red blood corpuscles, RBCs) of mammals [27]. When oxygen binds to a $\mathrm{Fe}$ ion in $\mathrm{Hb}$ (the oxidation state changes from $\mathrm{Fe}^{2+}$ to $\mathrm{Fe}^{3+}$ ), it pushes the $\mathrm{Fe}$ atom through the centre of the porphyrin plane and also, the protein subunits undergo conformational change as the strain of this change propagates, increasing the affinity for
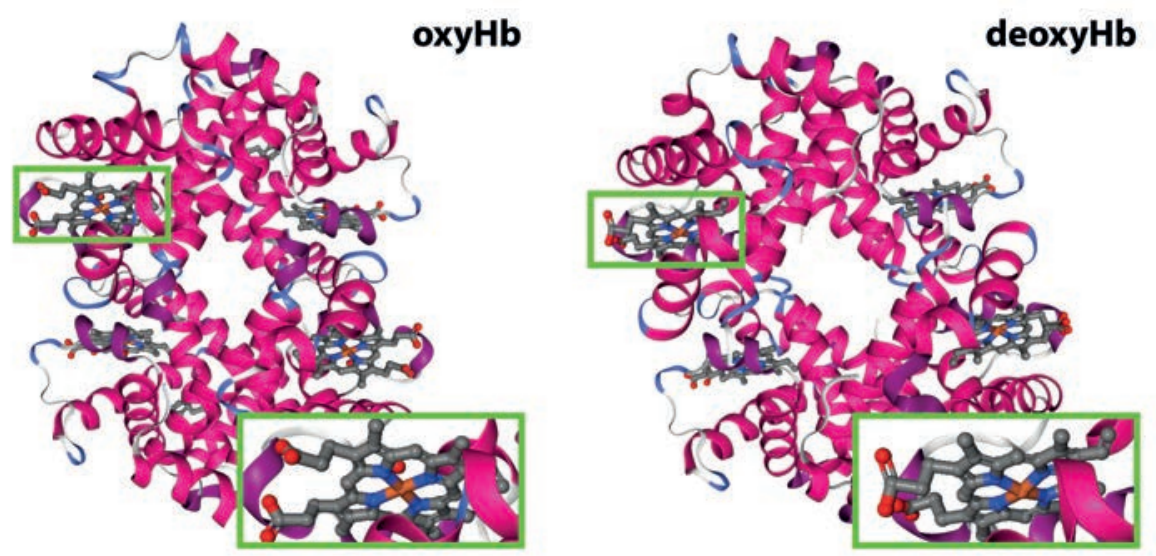

Figure 1.2: Structure of the haemoglobin complex at $1.25 \AA$ [28] with the four globin (red and blue helices) and the grey porphyrin rings with the Fe (orange) in the centre. The green inlay shows the $\approx 0.3-0.4 \AA$ shift of the Fe atom in the middle of the porphyrin ring due to binding of oxygen. 
oxygen at other haem sites. This property makes Hb very efficient as the principal oxygen transporter in mammalian blood. In 1845, Michael Faraday investigated the magnetic properties of dried blood and concluded that it did not exhibit magnetism [29], although interestingly enough, he noted in his diary "Must try recent fluid blood" but did not follow up. It would be nearly a century later, contemporaneously to Otto Stern's beam experiments, that Linus Pauling and Charles Coryell [30], building upon Faraday's work and notes, detailed the magnetic properties of the different forms of haemoglobin. Pauling and Coryell used a Gouy balance wherein they weighed samples in the presence and absence of a magnetic field generated using an electromagnet and the weights were measured at two different field strengths. In this classic experiment, they confirmed the previously known diamagnetism of the oxy and carbonmonoxy forms of $\mathrm{Hb}$ but discovered that the deoxy form was paramagnetic (due to the four unpaired electrons per Fe atom) [16]. Although Pauling and Coryell were unaware of its potential application, the difference in magnetic susceptibility properties of the different forms of $\mathrm{Hb}$ in arterial and venous blood would form the basis for functional MRI (fMRI) to be possible.

\subsection{Blood oxygenation and functional MRI}

In 1982, Thulborn and colleagues [31] showed that the transverse relaxation rate $\left(\mathrm{T}_{2}\right)$ is a marker of the oxygenation state of the haemoglobin and concluded that NMR can be used to determine blood oxygenation in vivo quickly, quantitatively and non-invasively. Building upon these findings, over the course of 1989-1990, Seiji Ogawa and colleagues demonstrated in rodents, that vessels with increased deoxy-Hb appear as dark lines in coronal slices and disappear under full oxygenation. Ogawa noted that this endogenous contrast is sensitive to blood oxygenation, 
higher contrast was observed with gradient echo (GE) acquisition schemes and that the contrast is echo time (TE) dependent. A gradient-echo is formed when the frequency-encoding positive gradient counterbalances the initial negative gradient and the signal relaxes with $R_{2}{ }^{*}$ (where $R_{2}{ }^{*}=R_{2}+R_{2}{ }^{\prime}$ ) whereas a spin-echo (SE) is formed by utilising an additional $180^{\circ}$ pulse at $\mathrm{TE} / 2$ to refocus the $\mathrm{T}_{2}$, thereby, being purely $\mathrm{T}_{2}$ [32]. In the first of several papers [33], Ogawa concludes, "When some region in a brain is much more active than other regions, the active region could show darker lines in the image because of the increased level of deoxyhaemoglobin resulting from higher oxygen consumption. Therefore, in addition to the anatomy of the brain, one aspect of its physiology can be studied by the MRI of water." ${ }^{2}$, foreshadowing the potential application of their observations. It was only in their seminal paper in PNAS [35] the same year that Ogawa and colleagues labelled this contrast as, Blood Oxygenation Level-Dependent (BOLD) and made the modest admission that the BOLD contrast is likely to be "complementary to PET" for functional neuroimaging. However, the discovery of the BOLD contrast proved to be the turning point for MRI as it soon leapt ahead of PET as the methodof-choice for non-invasive functional neuroimaging given the superior spatial and temporal resolution of the MRI technique. The rapid adoption and ubiquity of BOLD fMRI was due to the availability of fast imaging methods, specifically, EPI [14] and development of MR pulse sequences [32]. Soon after in 1992, three research groups realised the promise of BOLD for human fMRI in quick succession, all using gradient-echo MRI sequences [36-38 ${ }^{3}$. This would be the first of many

\footnotetext{
${ }^{2}$ It is important to note that the BOLD signal during stimulus presentation is an overall positive signal, contrary to what Ogawa and colleagues expected in their seminal 1990 paper. This enhancement of the fMRI signal in tissue during stimulation is driven by the oversupply of oxygenated blood arriving via increase in $\mathrm{CBF}\left(\gg \mathrm{CBV}\right.$ and $\mathrm{CMRO}_{2}$ ) that overcompensates for the oxygenation demand in the region [34].

${ }^{3}$ All the fascinating details of events leading up to the first human fMRI studies have been recounted by the researchers involved in Part I of fMRI: From Nuclear Spins to Brain Functions [40-42].
} 
brain mapping studies that would utilise the BOLD contrast for clinical and cognitive neuroscience applications.

\subsection{Physiological bases of functional MRI}

There is a vast body of literature describing the brain metabolism-blood flow coupling and a thorough review is beyond the scope of this thesis. However, a modest compromise would be a short discussion about the interplay of CBF, CBV, $\mathrm{CMRO}_{2}$ and the BOLD contrast during neural activation. The BOLD fMRI signal is directly determined by changes in CBV and blood oxygenation, where blood oxygenation is determined by $\mathrm{CBF}, \mathrm{CMRO}_{2}$ and $\mathrm{CBV}_{\text {deoxy }}$. Haematocrit is also another parameter to consider, however, it is only relevant for inter-subject comparisons as the intra-subject change due to activation is negligible. At the site of neuronal activation, increased metabolism $\left(\mathrm{CMRO}_{2}\right)$ leads to hypo-oxygenation and a consequent decrease in the fMRI signal. This is accompanied by an increase in $\mathrm{CBF}$ bringing oxygenated blood to the region resulting in hyper-oxygenation and thus, an increase in the fMRI signal, which is exactly what we observe following stimulus presentation, an overall positive BOLD response. While this seems relatively straight-forward, changes in CBV can cause increase or decrease of the fMRI signal depending on the acquisition parameters such as TE, field strength and sequence type [43]. Transients observed in the haemodynamic response result due to temporal mismatch of these physiological parameters during baseline, activation and deactivation. The BOLD response has some typical characteristics with an inconsistently observed early signal decrease (known as 'initial dip'), followed by a delayed increase (1-2 s) reaching the highest signal 5-10 s, followed by a return to baseline. Following the cessation of the stimulus, a signal decrease (known as 'post-stimulus undershoot') can be typically observed. The close asso- 


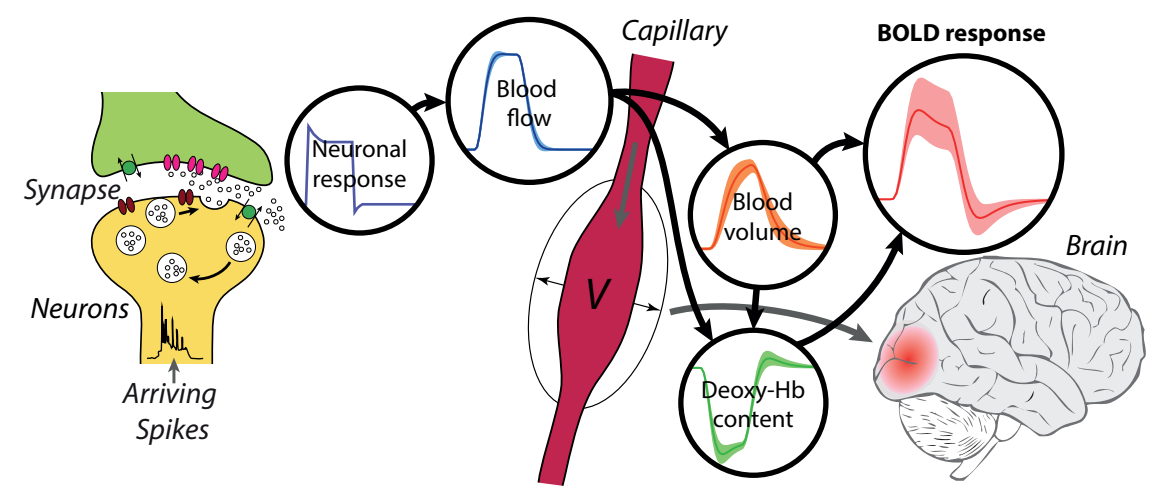

Figure 1.3: Physiological processes leading up to the BOLD signal. Neuronal events such as action potentials and post-synaptic activity consume oxygen $\left(\mathrm{CMRO}_{2}\right)$ and glucose $\left(\mathrm{CMR}_{g l c}\right)$ and neurovascular coupling results in increase in blood flow and volume [44] leading to changes in blood oxygenation (Figure courtesy of Dr. Martin Havlicek).

ciation of neuronal activity with changes in $\mathrm{CBF}$ is termed neurovascular coupling. The relationship between BOLD and different measures of neuronal activity was empirically determined in a seminal paper by Logothetis and colleagues [45], where they simultaneously measured BOLD and electrophysiological measures namely, multi-unit activity (MUA) and local field potential (LFP) reflecting population spiking activity and post-synaptic activity, respectively. They found a higher correlation of the BOLD signal and LFPs than with the MUA. In a follow up study Logothetis and colleagues [46] used serotonin to suppress the MUA leaving the LFP response intact and found that the BOLD response was unaffected, thereby, demonstrating the strong association between BOLD and post-synaptic activity. These experimental findings suggest that CBF-driven BOLD changes in a brain area is representative of input signals rather than output [45, 47, 48]. This can have implications for interpreting the fMRI data as the measured BOLD signal in one brain area could be caused remotely by input signals from a different 
area and therefore, may not spatially correspond to the regions with actual neuronal activity. Since the local post-synaptic activity in the spiking regions is much higher than the inter-areal input signals, it would not be unreasonable to assume that the BOLD signal measured in a region are associated with the local neuronal activity. Mapping CBF changes on the other hand could be more sensitive to areas with post-synaptic activity via feed-forward neurovascular coupling and not so much to regions with minimal post-synaptic activity (e.g. inter-areal). Spatial specificity of the measured signal, therefore, is an important factor for fMRI. That is, the fMRI signal should preferably arise from micro-vasculature (capillaries) contributions as they are in proximity to neuronal activation of interest. While this is the case with CBF fMRI, the GE BOLD signal from capillaries is small compared to the downstream BOLD signal arising from ascending (in GM, perpendicular to pial surface) and pial (draining) veins, therefore, is dominated by the venous signal $[43,49]^{4}$. As the strongest venous signal stems from pial vessels, for studies investigating layer-specific or column-specific changes, there is no substitute for imaging at a high spatial resolution to resolve the GM BOLD signal from that of pial vessels. And with very high spatial resolutions (typically sub-millimetre), the effect of the pial veins can be mitigated by reducing its partial voluming with neighbouring grey matter (GM). Although higher spatial resolution can address the GE-BOLD signal bias due to pial veins by reducing the GM partial voluming at the pial surface, the contribution of the intracortical ascending vein bias will remain. Addressing the latter bias requires detailed, accurate modelling approaches that are presently under development and the preliminary results [51] are very promising.

\footnotetext{
${ }^{4}$ For an in-depth review of this topic please see "Physiology and Physics of the fMRI Signal" by Uludağ and Uğurbil in fMRI: From Nuclear Spins to Brain Functions [50].
} 


\subsection{BOLD fMRI at ultra-high field}

Ever since the early days of fMRI, there has been a strong desire from the community to push to higher magnetic field strengths for both animal and human studies. Moving to ultra-high magnetic fields (UHF) (i.e. $\mathrm{B}_{0} \geq 7 \mathrm{~T}$ ) has considerable advantages such as increased functional contrast-to-noise (fCNR, [52]) and sheer gain in signal-to-noise (SNR, [53]). The gain in SNR due to stronger $\mathrm{B}_{0}$ will be diminished by going to higher spatial resolutions (sub-millimetre voxels) due to a reduction in number of water protons available in each voxel and the consequent increase in thermal noise contribution. With fMRI studies it is important to realise that the overall CNR can improve despite poorer image SNR as long as the temporal SNR (tSNR) is improved. This has motivated development of better hardware [54] and novel pulse sequences that allow fast, often three-dimensional, high-resolution imaging [55]. These developments directly benefited fMRI studies as the higher spatial resolution reduces partial voluming with WM, CSF and large surface vessels (low tSNR compartments), while faster imaging at UHF is a requirement due to the shortening of $\mathrm{T}_{2} / \mathrm{T}_{2}{ }^{*}$. The BOLD signal arises from a combination of both intra- and extra-vascular signals. It is now known that the intravascular $\mathrm{T}_{2}$ decreases in the presence of deoxy-Hb and changes quadratically with field strength [31]. Therefore, a signal change in BOLD-weighted images is observed when there is a change in deoxy- $\mathrm{Hb}$ associated shortening of the $\mathrm{T}_{2}$ of blood (intravascular BOLD) and will be present both in large and small vessels. The extravascular BOLD signal is induced by the deoxy-Hb associated magnetic susceptibility differences between the vessel and the relatively diamagnetic tissue surrounding it, and this also increases with $\mathrm{B}_{0}$ [43]. At UHF, the intravascular signal (blood $\mathrm{T}_{2} / \mathrm{T}_{2}{ }^{*}$ ) decays much faster than the surrounding tissue, and when the data is acquired at a TE that is approximately the GM $\mathrm{T}_{2}{ }^{*}$, the intravascular signal is almost com- 
pletely decayed. While SE is considered to be more tissue specific relative to GE [43, 52, 56], GE with an EPI readout is still considered the workhorse for highresolution $\mathrm{fMRI}$ at UHF. Due to implementation limitations with SE-EPI, there is no avoiding some $\mathrm{T}_{2}{ }^{*}$ weighting [57] which contribute spatially non-specific effects, taking away the principle selling point of SE whilst presenting challenges such as reaching the SAR limits quicker than with GE (due to the additional refocusing pulse in SE) and its inherently lower SNR, further limiting its accelerated imaging capabilities. On the other hand, the GE-BOLD contrast that is used for most fMRI studies, has unparalleled SNR gain in both micro- and macro-vascular compartments at UHF. The differential weighting of these macro- and micro-vascular compartments play an important role in the spatial fidelity of the GE-BOLD signal when we move to higher and higher spatial resolutions, the implications of which are discussed in the section below.

\subsection{Cortical layers and laminar fMRI}

A fundamental hypothesis in neuroscience is that the underlying cellular composition (cytoarchitecture) of a brain region plays a critical role in its function. A distinguishing feature of cytoarchitecture is the layered organisation of different neuronal cell types (analogous to geological layers) [58]. Based on Nissl stained sections Vogt [59] classified the cortex as isocortex (six layers) which contains all the sensory cortices and allocortex (having three or four layers, if at all) based on its underlying laminar cytoarchitecture. Brodmann described three cytoarchitectonic partitions of the occipital lobe, primary visual area (V1), secondary visual area (V2), and a tertiary visual area (V3). Primary sensory areas receive direct (dominantly) unimodal afferents from the thalamus and are reciprocally connected with higher order unimodal sensory areas [60]. The major part of V1 
receives afferents from both eyes and represents the central part of the visual field at the occipital pole, whereas the upper visual field is found solely on the lower bank and the higher visual field on the upper bank of the calcarine sulcus. The vertical meridian extends along the border between V1 and V2. The visual cortex shows a well differentiated laminar structure with a prominent layer IV (that can be further subdivided into sublayers IVa-c). It is this Layer IV (IVa and c, in particular) that receives parvo- and magnocellular afferents from the lateral geniculate nucleus (LGN) [60]. Layer IVb contains myelinated horizontal collaterals of stellate neurons, axon collaterals of layer III pyramidal neurons and feedback projections from areas V2 and MT/V5 forming the infamous, stria of Gennari. The cerebral micro-vasculature is also specialised around the laminar organisation of the cortex [62] making it possible to probe it using sub-millimetre spatial resolution fMRI at UHF. Such high spatial resolutions are necessitated by the fact that the human neocortex can be $\approx 2 \mathrm{~mm}$ thick (e.g. V1) and be convoluted making it difficult to discern layer-specific signals at resolutions $\geq 1 \mathrm{~mm}$. While fMRI research continued to focus on mapping, studying brain areas and inter-areal circuitry, the availability of UHF human MRI scanners has opened the door to non-invasive neuroscientific investigations at the intra-areal (laminar or columnar) circuitry in vivo, that were previously thought to be the realm of invasive animal electrophysiology.

The earliest laminar fMRI study in humans was carried out in 2007 at $3 \mathrm{~T}$ by Ress and colleagues [63]. At this time, fMRI at UHF ( $\geq 7 \mathrm{~T}$ ) was still very much in its infancy and the advantages of UHF for fMRI studies probing cortical layers was being studied. In 2009, Uludağ and colleagues [43] investigated the spatial specificity of the GE $\left(\mathrm{T}_{2} *\right)$ and $\mathrm{SE}\left(\mathrm{T}_{2}\right)$ signals at field strengths upto $16.4 \mathrm{~T}$ using an integrative BOLD model and the results had profound implications for lam- 

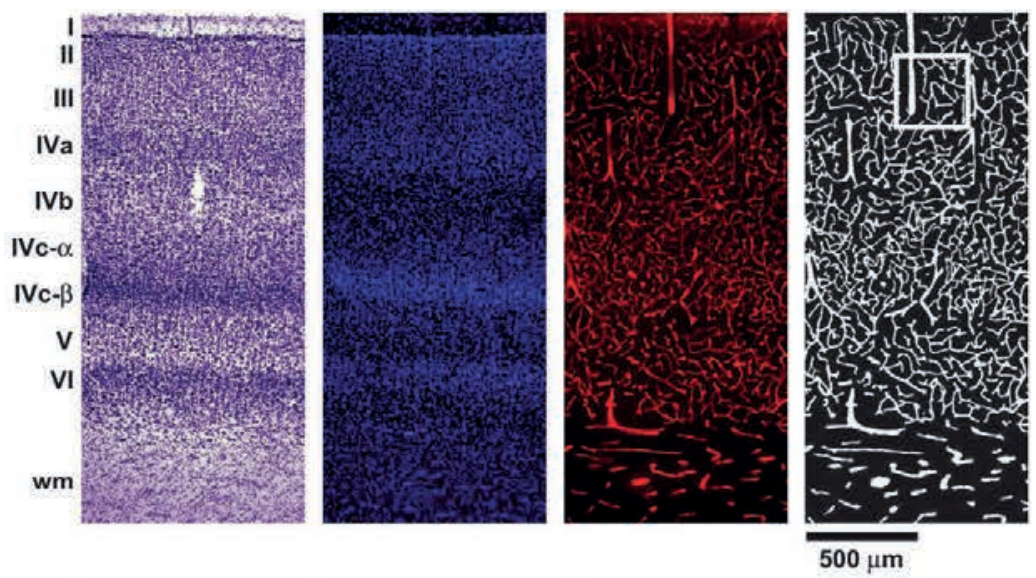

Figure 1.4: Adapted from [61] by Weber and colleagues. From left to right: Nissl, DAPI and Cy-3 anti-collagen type IV stained sections of the macaque striate cortex for visualising neuronal cell bodies and the quantification of the vessels. The last image is the processed vessel image (same as the third).

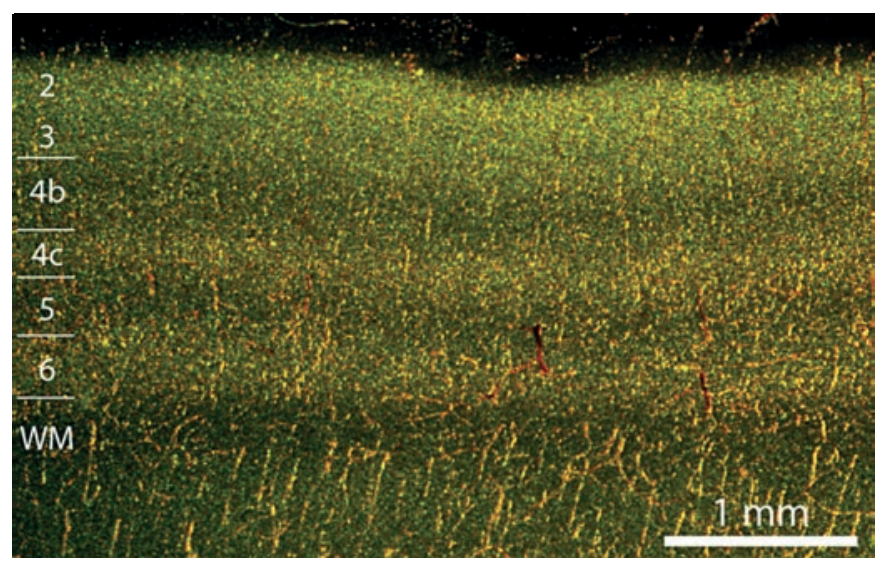

Figure 1.5: Adapted from [62] by Adams and colleagues. The figure shows that the layer-specific vascular perfusion is correlated to the neuronal density in the macaque striate cortex. The golden orange colour indicates the endogenous peroxidase activity in red blood cells with the strongest vascular signal coming from layers $2,4 \mathrm{c}$, and 6 whereas the weakest signal from layers $1,4 \mathrm{~b}$, and 5 . 
inar fMRI at UHF. In this study, Uludağ and colleagues mention that while its possible to perform laminar fMRI at $3 \mathrm{~T}$, it often requires high-resolution isotropic voxels with a segmented GRE readout, acquired using a surface coil and analysis that possibly avoids the pial vessels. More importantly, Uludağ and colleagues [43] showed that the largest micro-vascular (local vascular units in activated region i.e., arterioles/venules/capillaries) specificity could be achieved using spinecho at field strengths between $4-9.4 \mathrm{~T}$ due to (a) the increased extra-vascular BOLD contribution to the total fMRI signal and (b) reduced intra-vascular BOLD contribution from the veins (macro-vasculature) [43]. At $\mathrm{TE} \approx$ tissue $\mathrm{T}_{2}{ }^{*}$ and high spatial resolutions, most voxels will capture the extra-vascular signal from dense capillary beds in the tissue and not from the far fewer large pial vessels. In GE acquisitions, the BOLD signal changes associated with the large vessels are not closely correlated with site of activation but can contribute non-specific signal changes. While spill-over signal from the vessel into the surrounding tissue is a problem, due to the unparalleled SNR of GE, selectively excluding regions with large vessels from analysis [64] has been shown to yield reproducible results [65] comparable to SE albeit with lower spatial fidelity. It would only be a matter of time before laminar fMRI would be carried out at 7 T. In 2010, Polimeni and colleagues [66] presented novel whole-brain surface-based tools for laminar analysis at $7 \mathrm{~T}$ using GE-EPI at $1 \mathrm{~mm}$ isotropic spatial resolution and show that sampling the GE-BOLD signal away from the pial surface has improved spatial fidelity and shows that depth-dependent investigations using GE-BOLD is feasible. It was soon followed by the first sub-millimetre laminar fMRI study (0.75 $\mathrm{mm}$ isotropic) by Koopmans and colleagues [67] using GE-FLASH who had previously shown the feasibility of laminar fMRI at $3 \mathrm{~T}$ [68]. These early studies showed that although the cyto-architectonic definition of layers are beyond the resolving power of MRI, it 
is possible to study the layer-specific fMRI signal changes using computationally constructed cortical depths or laminae [69-71], interpolation [66, 67] and more recently, spatial GLM [72-74] techniques.

The field has grown since 2010 with the increasing availability of UHF scanners (presently over sixty $7 \mathrm{~T}$ scanners in the world), data analysis tools [75, 76], advances in pulse sequences that take advantage of parallel imaging [55], all of which further the possibilities for high-resolution fMRI studies. A detailed list of the 7T laminar fMRI studies till date has been tabulated in Table 1.1 at the end of this section. Till date $\approx 73 \%$ of all laminar fMRI studies have focussed on the visual cortex, followed by $\approx 17 \%$ motor cortex and $\approx 10 \%$ the auditory cortex (Fig. 1.6). Unsurprisingly, a majority $(\approx 65 \%)$ of these studies employ GE BOLD followed by $\approx 15 \%$ use CBV-weighted vascular space occupancy (VASO) (Fig. 1.7). Although

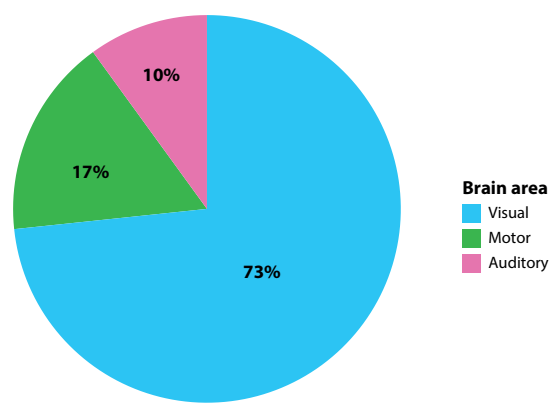

Figure 1.6: Pie chart illustrating the distribution of laminar fMRI studies carried out in sensory areas.

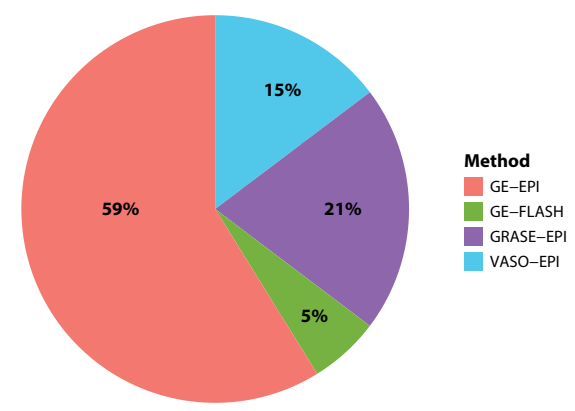

Figure 1.7: Pie chart illustrating the sequence preferences in currently published laminar fMRI studies. $\approx 20 \%$ of the studies report using a $\mathrm{T}_{2}$ weighted gradient- and spin-echo (GRASE), not all of them use it for the laminar investigation per se (e.g. [77]). It must be noted that the CBF contrast has not been used for laminar fMRI in humans at 7T (but see Chapter 4 for details). 
The anatomical data on the other hand follows a more expected trend with a majority $(\approx 69 \%)$ of studies acquiring data that is differently-distorted compared to the functional, typically with $\mathrm{T}_{1}$-weighting, followed by $\approx 22 \%$ studies acquiring distortion-matched $\mathrm{T}_{1}$ and $\approx 9 \%$ acquiring distortion-matched $\mathrm{T}_{2}^{*}$. The work presented in this thesis addresses some of the acquisition and analysis considerations for BOLD and CBF fMRI to enable layer-specific investigations of the human visual cortex at UHF. 


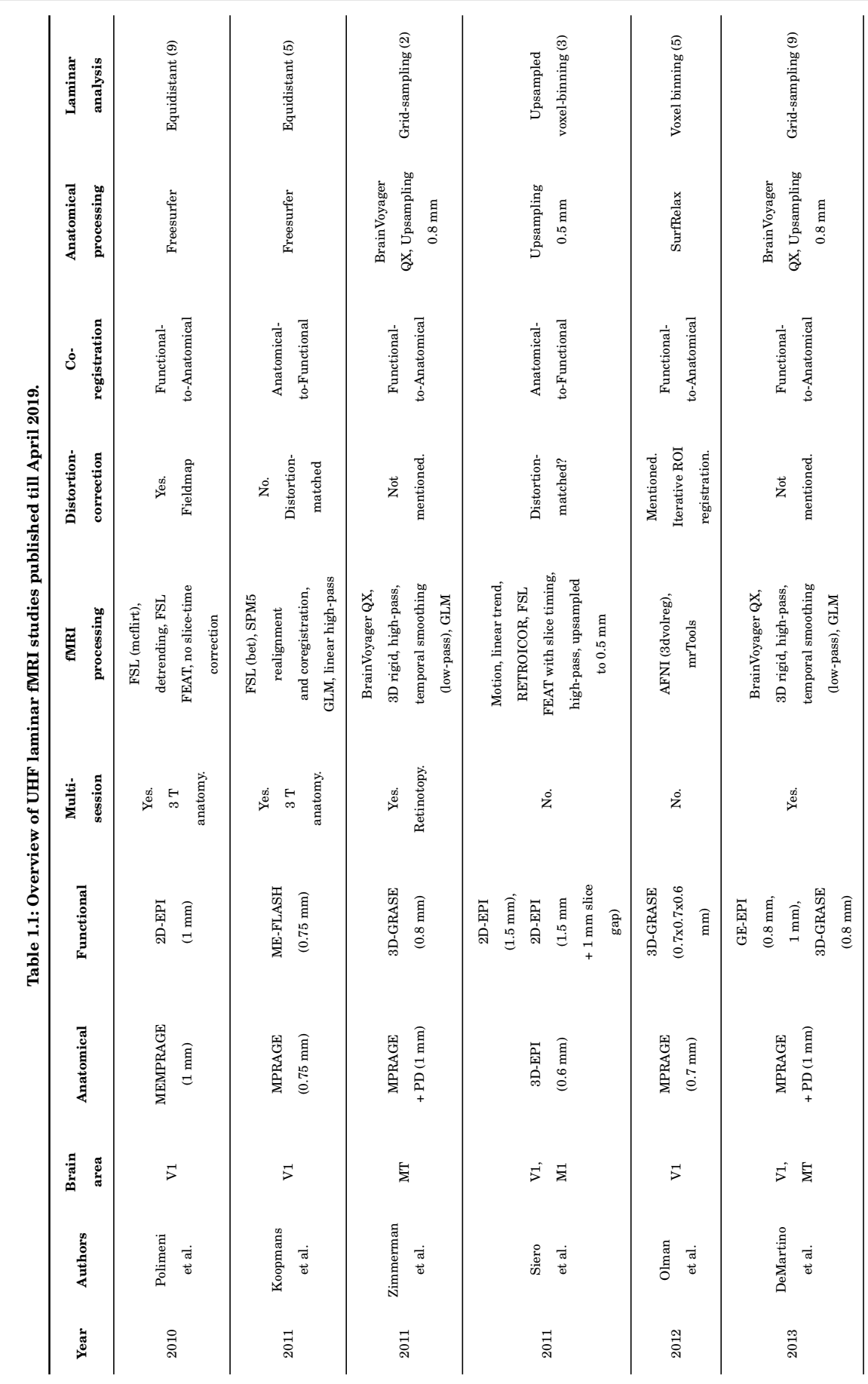




\begin{tabular}{|c|c|c|c|c|}
\hline 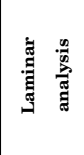 & 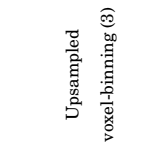 & 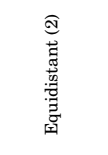 & 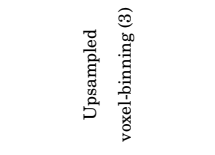 & 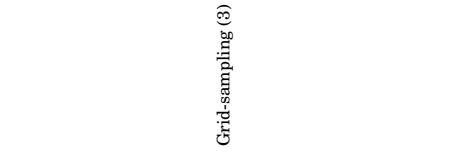 \\
\hline 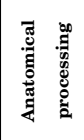 & 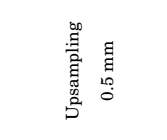 & 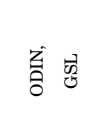 & 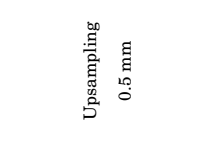 & 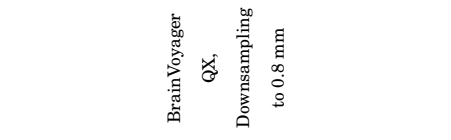 \\
\hline o & 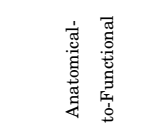 & $\overleftrightarrow{\underline{z}}$ & 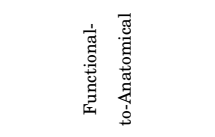 & 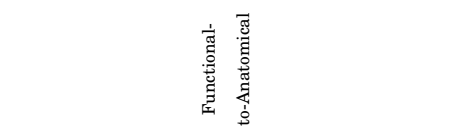 \\
\hline 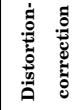 & 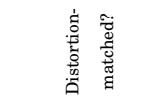 & $\dot{z}$ & 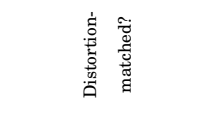 & 㟥兽 \\
\hline 量 & 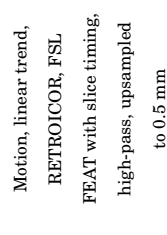 & 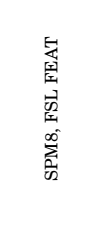 & 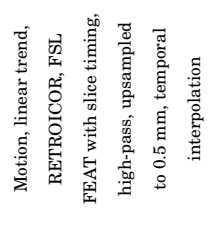 & 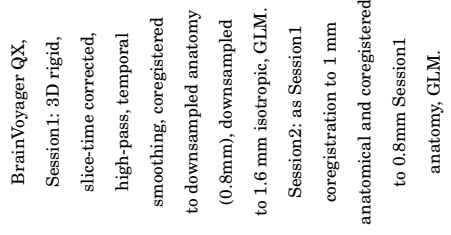 \\
\hline 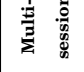 & 宅 & $\ddot{\Delta}$ & $\ddot{z}$ & 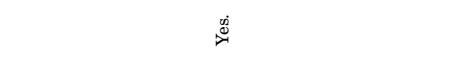 \\
\hline 范 & 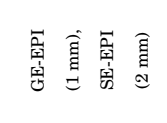 & 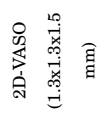 & 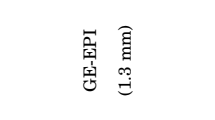 & 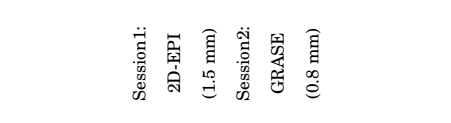 \\
\hline 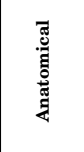 & 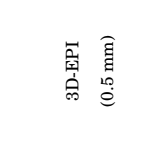 & $\overleftrightarrow{\bar{z}}$ & 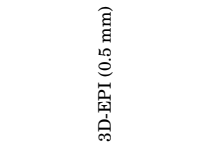 & 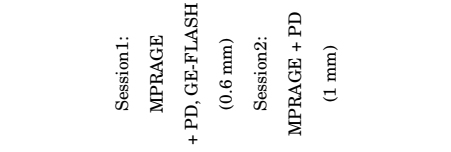 \\
\hline 菢 & 5 & 5 & 5 & द \\
\hline 要 & 离 & 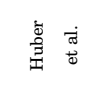 & 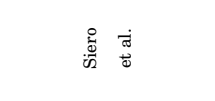 & 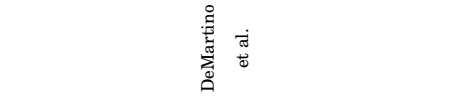 \\
\hline 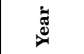 & : & 芯 & 量 & 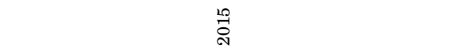 \\
\hline
\end{tabular}




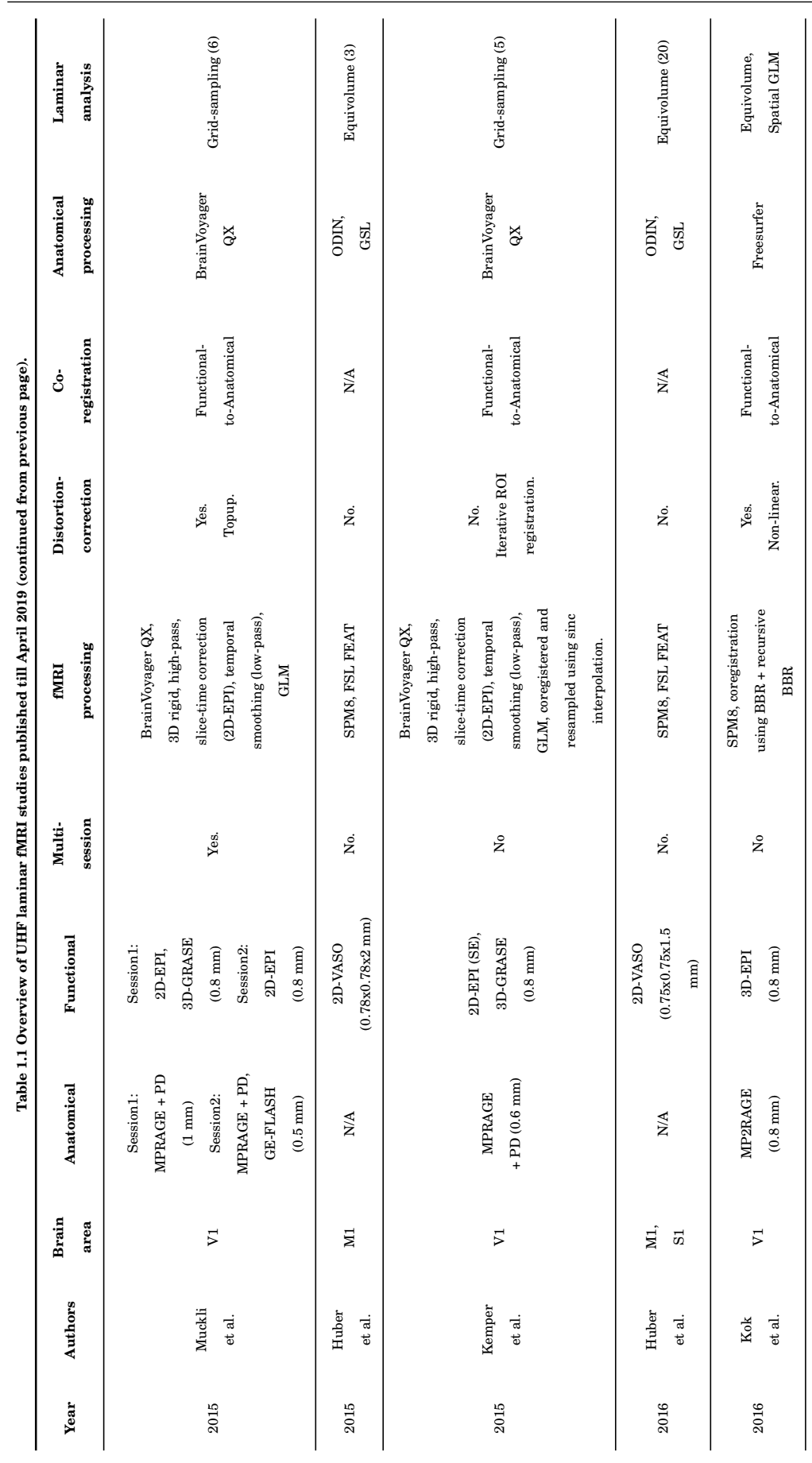




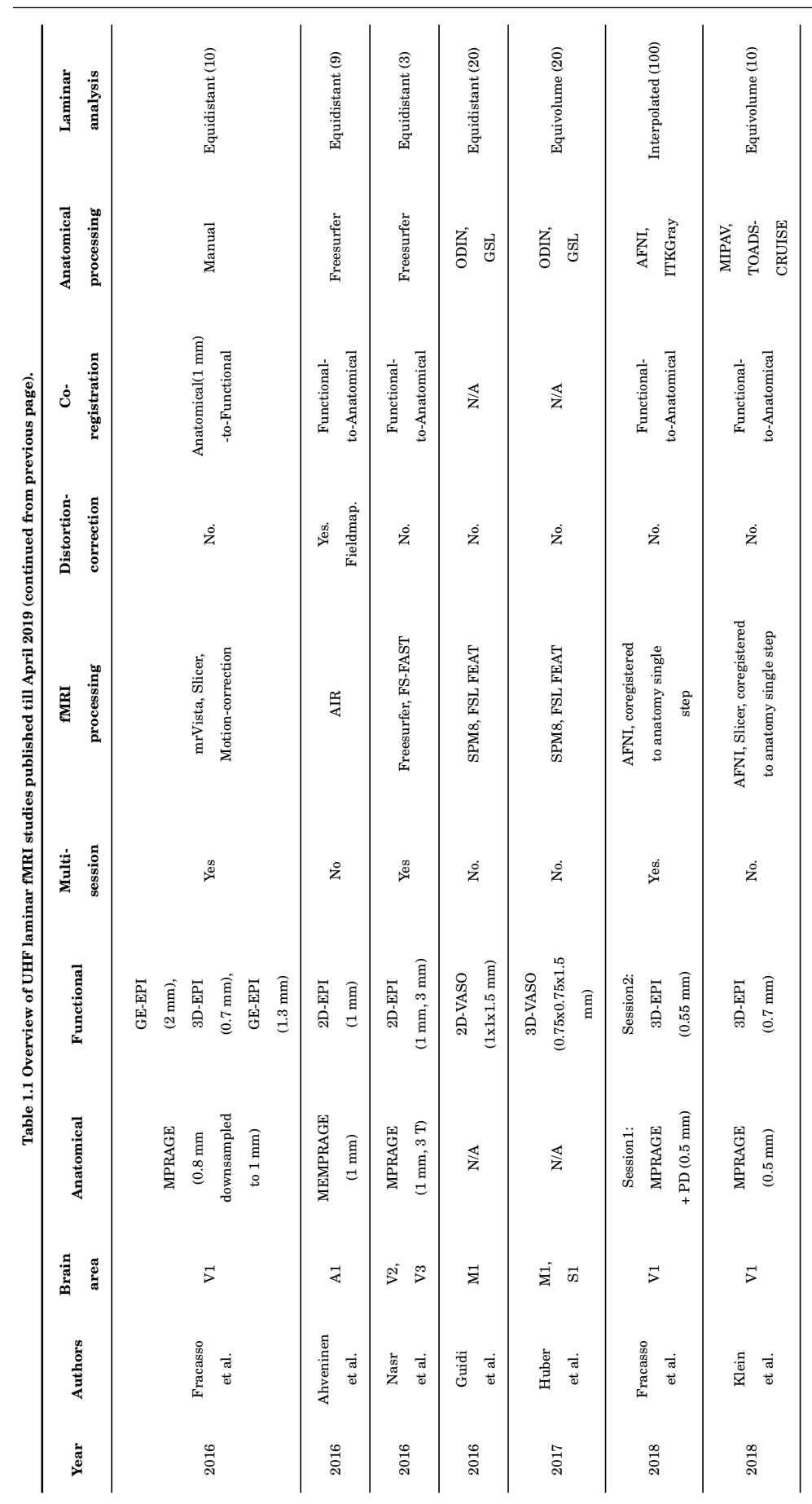




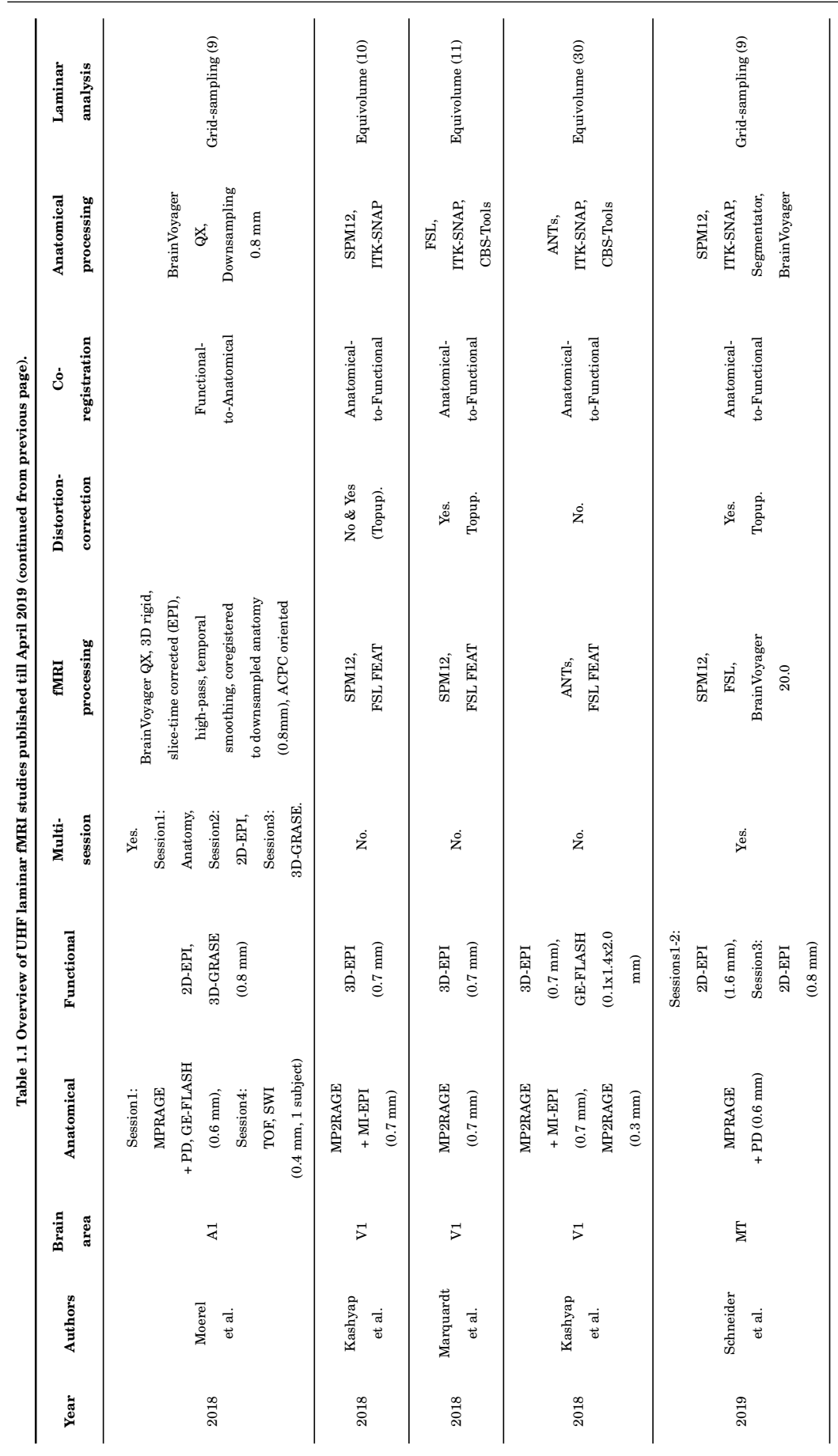




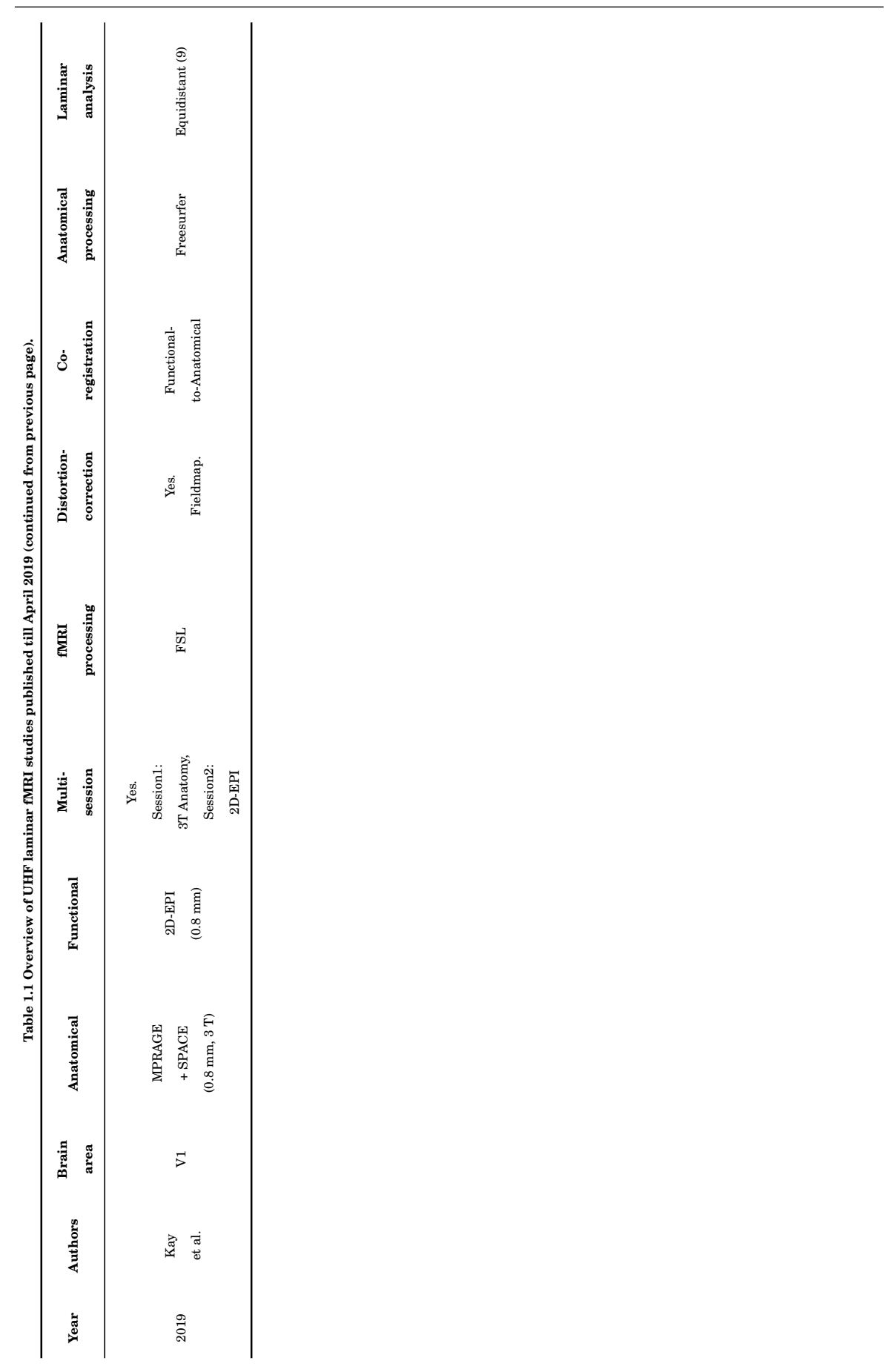




\subsection{Structure of the thesis}

fMRI data acquired with an EPI read-out is more strongly affected by local variations in magnetic susceptibility (e.g. at tissue-air interface) at UHF resulting in strong geometric distortions along the phase-encoding direction [78]. Most laminar BOLD fMRI studies so far have relied on the conventional fMRI acquisition and analysis approach using differently-distorted structural and functional datasets, and transforming the functional data into the space of the anatomical data. While this is standard procedure in $\leq 3 \mathrm{~T}$, it is crucial for high-resolution fMRI studies that the data are minimally processed with particular focus on highaccuracy of co-registration and segmentation.

In Chapter 2, we address the need of a minimal processing workflow for laminar fMRI studies and demonstrate the feasibility of working with high-resolution partial brain coverage, spatially un-distorted and distorted anatomical datasets. We characterise the impact of distortion-correction and its associated misregistration on the measured layer-specific BOLD signal and provide an analysis framework for laminar fMRI studies utilising a distortion-matched quantitative $\mathrm{T}_{1}$ EPI instead of traditional anatomical datasets. We experimentally show that the BOLD post-stimulus undershoot is strongest in tissue not veins. Finally, we discuss vascular biases in GE-BOLD and propose methods to possibly address it.

While UHF fMRI enables probing neuroscientific questions at the mesoscopic scale in humans, the highest spatial resolution achieved for laminar fMRI was $\approx 0.7-0.8$ $\mathrm{mm}$ isotropic. This is still large compared to the spatial dimensions of cortical layers and columns and as a consequence, the functional data are typically spatially scaled up using various interpolation techniques and averaged over an extended region-of-interest (ROI) to sample the underlying layer-specific fMRI signal. As discussed previously, there is no substitute for high spatial resolution when it 
comes to laminar fMRI. In animals however, a so-called line-scanning fMRI [79] technique allows sampling laminar signal at an unprecedented spatial $(50 \mu \mathrm{m})$ and temporal $(50 \mathrm{~ms})$ resolution. While this is desirable, it is currently not possible in humans to obtain both high spatial $(\approx$ sub-millimetre) and temporal $(\approx$ subsecond) resolution fMRI data with sufficient SNR. Given that the haemodynamic response is sluggish [45], achieving high temporal resolution may not be necessary in most applications whereas optimising acquisitions for higher spatial resolutions along the dimension of interest (i.e. radial or tangential directions) can be beneficial for laminar or columnar fMRI.

In Chapter 3, we showcase a novel laminar fMRI acquisition strategy that pushes this boundary of spatial resolution further by acquiring data at an unprecedented $0.1 \mathrm{~mm}$ spatial resolution along the laminar direction while being highly anisotropic along the other two dimensions $(1.4 \mathrm{~mm} \times 2.0 \mathrm{~mm})$. We compare the novel anisotropic approach to the (optimised) conventional EPI approach (developed in Chapter 2) and demonstrate the feasibility of using the anisotropic acquisitions strategy to obtain laminar (and columnar) BOLD signal in the human visual cortex. We also show, both experimentally and using simulations, the differences in effective spatial resolution of the two approaches and that the laminar BOLD signal profile, even over short patches of cortex, is not uniform and argue in favour of the biophysical source of this variability. In addition, we apply the anisotropic approach for studying columnar organisation of the cortex at $0.1 \mathrm{~mm}$ along the tangential direction.

Despite the ubiquitous use of BOLD fMRI, the approach has shortcomings such as the fact that it is difficult to extract a quantitative physiological parameter from the observed signal changes. However, there are non-BOLD alternatives that allow functional mapping such as measuring CBF or CBV [80] which are quantita- 
tive physiological parameters. While CBV fMRI using the VASO technique [81] is gaining widespread acceptance $[82,83]$ for laminar fMRI, functional CBF mapping with arterial spin labelling (ASL) has remained a challenge due to its poor sensitivity and low SNR [84]. However, CBF is more closely associated with neuronal activation than the BOLD [85] and VASO signals, and due to the nature of its signal, is less likely to suffer arterial and venous biases [86], therefore, is of great interest for laminar fMRI.

In Chapter 4, building upon previous work [84, 87, 88] we show for the first time that robust single-subject single-session high-resolution activation maps for CBF fMRI can be obtained at $7 \mathrm{~T}$ with sufficient brain coverage. We demonstrate that functional activation estimated using CBF and BOLD contrasts have different spatial spreads and distinct laminar profiles. We show that CBF mapping is a promising technique for laminar fMRI at UHF due to its quantitative nature and better tissue specificity i.e., does not suffer from the venous biases that plague GE-BOLD. 



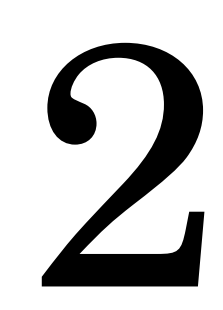

Impact of acquisition and analysis strategies for laminar fMRI

Associated publication: S. Kashyap, D. Ivanov, M. Havlicek, B. A. Poser, and K. Uludağ. "Impact of acquisition and analysis strategies on cortical depth-dependent fMRI". in: Neuroimage 168 (2018), pp. 332-344. 



\section{Abstract}

Functional MRI at ultra-high magnetic fields $(\geq 7 \mathrm{~T})$ provides the opportunity to probe columnar and laminar processing in the human brain in vivo at submillimetre spatial scales. However, fMRI data only indirectly reflects the neuronal laminar profile, due to a bias to ascending and pial veins inherent in gradient- and spin-echo BOLD fMRI. In addition, accurate delineation of the layers is difficult, due to the relatively large voxel sizes and lack of sufficient tissue contrast in the functional images. In conventional laminar fMRI studies, anatomical and functional data are acquired with different image read-out modules, the fMRI data are distortion-corrected and vascular biases are accounted for by subtracting the laminar activation profiles of different stimulus conditions. In this study, using high-resolution gradient-echo fMRI data $(0.7 \mathrm{~mm}$ isotropic) of the human visual cortex, we propose instead, that laminar functional information is best preserved if data analysis is performed in the original functional data space. To achieve this, we acquired anatomical images with high tissue contrast and similar distortion to the functional images using multiple inversion-recovery time EPI, thereby eliminating the need to un-distort the fMRI data. We demonstrate higher spatial accuracy for the cortical layer definitions of this approach as compared to the more conventional approach using MP2RAGE anatomy. In addition, we provide theoretical arguments and empirical evidence that vascular biases can be better accounted for using division instead of subtraction of the laminar profiles. Finally, we show that the haemodynamic response of grey matter has relatively stronger post-stimulus undershoot than the pial vein voxels. In summary, we show that the choice of fMRI data acquisition and processing can impact observable differences in the laminar profiles and present evidence that cortical laminar modulation of the BOLD signal can be resolved using gradient-echo imaging. 



\subsection{Introduction}

Functional magnetic resonance imaging (fMRI) has typically been utilised for localizing brain areas involved in processing cognitive and/or sensory tasks or in resting-state activity (recent overviews, see Uludağ, Uğurbil, and Berliner [39], and chapters within). The spatial resolutions of these mapping studies are usually between $8 \mathrm{~mm}^{3}$ and $64 \mathrm{~mm}^{3}$ (i.e. $2-4 \mathrm{~mm}$ along each voxel dimension). Given that the human cortex is about $3 \mathrm{~mm}$ thick [58, 90], studying neuronal processing within the six cellular layers of the cortex is usually not feasible at field strengths of $3 \mathrm{~T}$ and below (but see Koopmans et al. [68], Ress et al. [63]). With the advent of ultra-high magnetic field (UHF) human MRI scanners, i.e., $7 \mathrm{~T}$ and above, early studies have achieved spatial resolutions below $1 \mathrm{~mm}$ in-plane and above $1 \mathrm{~mm}$ slice thickness [91, 92] and, recently, several studies have been published utilizing sub-millimetre isotropic voxels [73, 77, 93-99]. In the context of cortical laminar fMRI, high-resolution imaging at UHF is crucial to reduce partial voluming of grey matter (GM) voxels with white matter (WM) and cerebrospinal fluid (CSF) [56, 63, 66, 67, 100, 101], leading to a paradigm shift in the type of neuroscientific questions that can be investigated in vivo in humans. Recent studies have probed the columnar and laminar organization of the cortex [60], previously only possible in animal models, by acquiring not only the anatomical but also the functional MRI in humans at a sub-millimetre scale [73, 77, 93-99]. Please note, the term fMRI will refer to blood oxygenation level-dependent (BOLD) [33, 35] fMRI using gradient echo, unless other functional contrasts, such as cerebral blood volume (CBV) [102] or cerebral blood flow (CBF) [37], are explicitly mentioned.

In addition to the increased accessibility to UHF scanners, advances in MRI technology such as RF-coil technology (recent overviews, see Vaughan and Griffiths 
[103], and references therein), parallel imaging techniques [104-106] and optimizations of multi-modal MRI sequences [88, 96] have enabled researchers to push the spatial and temporal boundaries of fMRI. fMRI at UHF takes advantage of the substantial gain in image and temporal signal-to-noise (SNR) [107], increased microvascular BOLD signal for both gradient-echo (GE) and spin-echo (SE) contrasts [43] and sub-millimetre spatial resolution to better localise the hemodynamic signal in the cortex [108]. Nevertheless, both the acquisition and analysis of such high-resolution data are still being developed and optimised to unravel laminar fMRI signals.

fMRI acquisitions probe neuronal activity indirectly via induced vascular changes and it has been experimentally and numerically shown that both GE and SE have contributions from surface pial and intra-cortical ascending veins (see Uludağ and colleagues [43], and references therein). A dynamic biophysical model is needed to remove the vascular bias present in GE and SE data and successfully disentangle the neural from the vascular contributions. Modelling efforts are underway to address this issue in the context of laminar fMRI $[109,110]$. In the absence of a comprehensive model-driven approach, differences in the fMRI signal between stimulus conditions (i.e. subtraction) have been considered to faithfully represent the underlying electrophysiological profiles [98, 111-115].

In laminar GE-fMRI, there are two main vascular biases, a) draining of deoxygenated haemoglobin (deoxy-Hb) from lower to upper layers of the cortex via ascending veins, and b) baseline venous $\mathrm{CBV}\left(\mathrm{V}_{0}\right)$ and relaxation parameters, such as $\mathrm{T}_{2}{ }^{*}$, which can be different at the different depths. The first bias (draining deoxy- $\mathrm{Hb}$ ) is approximately an additive component to the local micro-vasculature signal (see Uludağ et al. [43]). That is, the total BOLD signal of a given layer consists of the local micro-vasculature- and the ascending vein-signals. The second 
bias (combination of $\mathrm{V}_{0}$ and other layer-specific baseline parameters) is an indicator for the layer's sensitivity for activation, i.e. the higher the baseline CBV value, the higher is the amount of deoxy-Hb in that layer and the higher is the BOLD signal for the same neuronal activity changes. Here, we propose to reduce the latter bias by dividing laminar signals between conditions and/or time-points [49]. Below, we provide a detailed theoretical justification of the "division" approach and provide evidence that division, rather than subtraction, better accounts for the vascular biases in the GE fMRI signal.

fMRI data, usually acquired with an echo-planar imaging (EPI) read-out, is strongly affected by local variations in magnetic susceptibility (e.g. at tissue-air interface) resulting in geometric distortions [78], which are most pronounced in the phaseencoding direction. In contrast, the anatomical data, typically acquired with a $\mathrm{T}_{1}$-weighting using a Magnetization Prepared RApid Gradient-Echo (MPRAGE) [116], or with additional quantitative $\mathrm{T}_{1}$ using a Magnetization Prepared 2 Rapid Acquisition Gradient Echoes (MP2RAGE) [117], have comparatively lesser distortions. The main reason to utilise a $\mathrm{T}_{1}$ anatomy for cortical layer definitions is because of the higher tissue contrast between GM, WM and CSF compared to EPI-fMRI data. Therefore, most laminar BOLD fMRI studies so far have relied on the conventional acquisition approach using a differently-distorted structural and functional datasets, such as: MEMPRAGE \& EPI [66], MPRAGE \& 3D-GRASE [77, 93, 98, 99], MPRAGE \& EPI [77, 95, 118], or MP2RAGE \& EPI [73]. Alternatively, laminar functional data has been acquired distortion-matched to the MPRAGE [67] using a GRE 3D-FLASH readout but at the cost of much lower fMRI temporal resolution compared to an EPI acquisition. Therefore, similar to low spatial resolution studies, the standard high-resolution neuroimaging dataset for laminar fMRI also consists of functional and anatomical data ac- 
quired with different pulse sequences, encoding schemes and readouts. Consequently, the different geometric distortions pose limitations on achieving accurate coregistration which is critical in studies probing the cortical micro-circuitry using high-resolution laminar fMRI. Several techniques have been developed to "distortion-correct" the functional data, thereby, making it geometrically similar to the anatomical reference (see Jezzard [119], and references therein). Commonly used methods for distortion-correction (apart from non-linear image registration) use a $B_{0}$ field map-based [120] or opposite phase-encoded EPI image-based [121] unwarping of the functional data [122]. Limitations of distortion-correction methods, such as blurring of the signal due to smoothing, loss in effective spatial resolution due to sub-voxel shifts [123], reduced fidelity of the fMRI due to incomplete recovery of spatial information in distorted regions [124] and imperfect distortioncorrection can additionally result in registration errors with the anatomical reference.

One of the outstanding problems for laminar fMRI is the spatially accurate anatomical definition of the cortical laminae. For the purposes of accurate anatomical definition of laminae, the anatomical image can be distorted either through postprocessing or by modifying the acquisition. For example, EPI, with an identical readout as the functional data, has been described to be a suitable method for fast $\mathrm{T}_{1}$ mapping using an inversion recovery preparation [125-130] for anatomical imaging at field strengths $\leq 3 \mathrm{~T}$ and recently, with multiple inversion-recovery time EPI (MI-EPI) at $7 \mathrm{~T}$ [131]. Contrary to the conventional data acquisition approaches, using MI-EPI can yield high tissue contrast similar to MP2RAGE but with the advantage of being distortion-matched to the fMRI data. Since high accuracy registration and minimal post-processing of the functional data is of paramount importance for laminar fMRI studies, a clear advantage of the MI-EPI 
approach is the opportunity to forego the need to distortion-correct the fMRI data and potentially achieve higher registration accuracy with the distortion-matched anatomical reference. In addition, this approach limits the potential blurring associated with distortion-correction and hence, the reduction of laminar-specific information. Although the MI-EPI approach offers a very promising alternative, its performance with respect to laminar fMRI analysis has not yet been quantitatively compared to other acquisition approaches.

The present study compares two workflows for analyzing laminar fMRI data, namely, the MI-EPI workflow (analysis in native EPI space i.e. fMRI EPI data and MI-EPI $\mathrm{T}_{1}$ ) to the Conventional workflow (analysis in anatomical space i.e. distortion-corrected fMRI EPI and MP2RAGE) for sub-millimetre laminar fMRI at 7 T. We present a processing workflow for laminar analysis of GE-EPI functional data using a distortion-matched anatomy acquired with MI-EPI and demonstrate that the MI-EPI $\mathrm{T}_{1}$ can be used to accurately define laminars in the native EPI space.

In this study, we also propose that the laminar specificity of the GE signal can be improved by normalising the spatial profile of contrasting stimulus conditions with each other or considering the transients of the hemodynamic response, such as the ratio of the post-stimulus undershoot to the positive BOLD signal [132]. Although the GE-fMRI signal is limited in its spatial specificity [43, 52, 57, 94, 133] due to an inherent bias to draining veins both intra-cortically and on the pial surface, it remains a popular approach to acquire functional data in laminar studies $[66,73,77,95,118]$. Since the GE signal change is expected to be the largest along the pial surface and around large veins, we also investigated the dynamics of the event-related average time-course from CSF and venous voxels. Lastly, given the mesoscopic scale of the cortical circuitry being probed using laminar fMRI, we in- 
vestigated how the size of the region-of-interest (ROI) affects the sampled laminar GE-EPI signal. In short, this study presents the current state-of-the-art in acquisition and practical considerations for the analysis of high-resolution laminar BOLD fMRI data at ultra-high magnetic fields.

\subsection{Methods}

Five healthy participants (two female, median age 28 years) with normal or correctedto-normal vision participated in this study. Written informed consent was obtained and participants were duly compensated for their participation. The procedures were conducted with prior approval from the Ethical Committee of the Faculty of Psychology and Neuroscience at Maastricht University.

\subsubsection{Experimental paradigm}

Each run consisted of either static or flickering checkerboards as stimuli, interspersed with isoluminant grey screen as resting condition. The experimental runs alternated between static and flickering stimuli. The static (Michelson contrast 1) and flickering ( $\approx 8 \mathrm{~Hz}$, Michelson contrast $1 / 3)$ checkerboard hemi-annuli (Fig. 2.1) were designed based on an earlier study [134] to induce the same positive BOLD (PB) signal amplitude but eliciting a larger post-stimulus undershoot (PSU) for the flickering compared to the static stimulus [135]. The stimuli were created and presented to the left visual hemi-field using PsychoPy [136] in a block design for $20 \mathrm{~s}$ followed by $40 \mathrm{~s}$ of rest, with each of the six runs consisting of ten blocks of stimulus and rest. The initial baseline resting duration was $40 \mathrm{~s}$, resulting in a total duration of $640 \mathrm{~s}$ for each run. During the scans, participants were asked to remain motionless and fixate on a central white fixation 


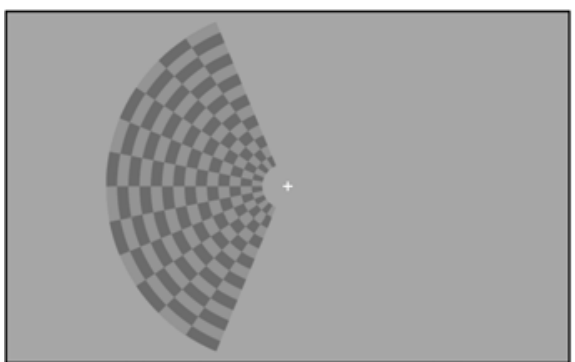

FLICKER

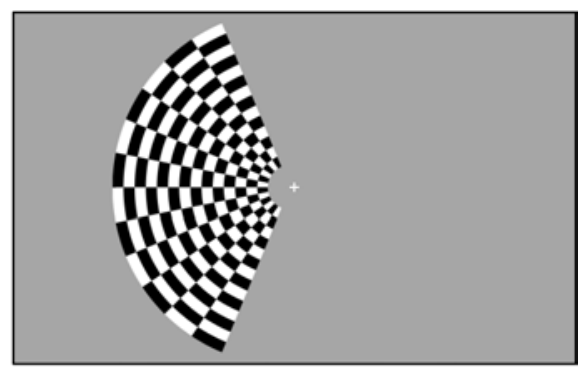

STATIC

Figure 2.1: Reduced contrast flickering (left) and full contrast static (right) checkerboard stimuli used in the present study.

cross.

\subsubsection{Data acquisition}

All data were acquired on a whole-body Siemens Magnetom $7 \mathrm{~T}$ research scanner (Siemens Medical Solutions, Erlangen, Germany) using a 32-channel phasedarray head coil (Nova Medical, Wilmington, MA, USA). Sequence parameters, described below, were optimised in pilot experiments.

\section{Anatomical data}

MP2RAGE: Whole-brain quantitative $\mathrm{T}_{1}$ images were obtained at $0.7 \mathrm{~mm}$ isotropic resolution using a 3D-MP2RAGE sequence [117] (240 sagittal slices; GRAPPA $=3$; partial-Fourier phase $=6 / 8 ;$ Ref. $\mathrm{PE}$ lines $=24 ; \mathrm{TR}=5000 \mathrm{~ms} ; \mathrm{TI}_{1} / \mathrm{TI}_{2}=$ $900 / 2750 \mathrm{~ms} ; \alpha_{1} / \alpha_{2}=5^{\circ} / 3^{\circ}$; phase-encoding= $\mathrm{A} \gg \mathrm{P}$ ) and was followed by a wholebrain $\mathrm{B}_{1}{ }^{+}$map using an Sa2RAGE sequence [137, 138] $\left(\mathrm{TR}=2400 \mathrm{~ms} ; \mathrm{TI}_{1} / \mathrm{TI}_{2}=\right.$ $58 / 1800 \mathrm{~ms} ; \alpha_{1} / \alpha_{2}=4^{\circ} / 10^{\circ}$ ). 

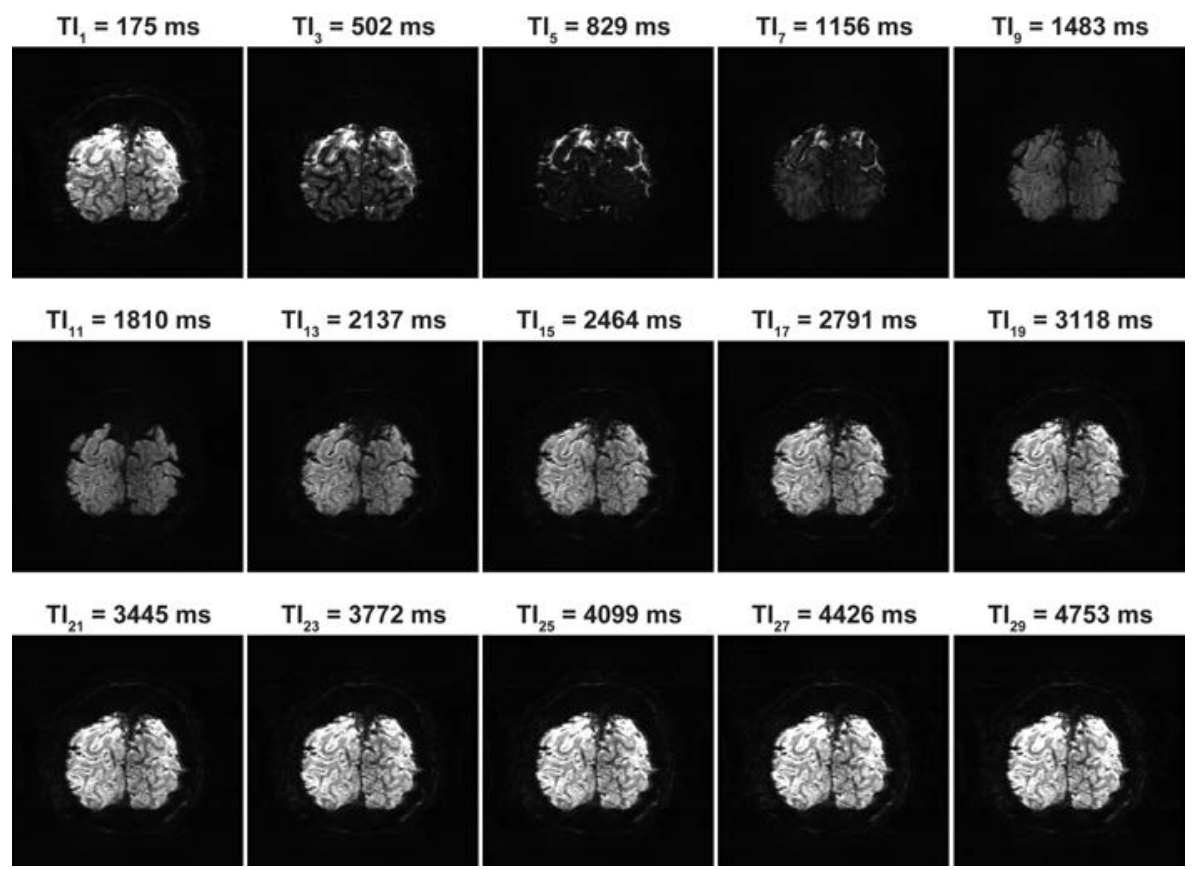

Figure 2.2: A representative slice from a single subject acquired with different inversion times in the MI-EPI protocol (15 out of 30 TIs presented for illustrative clarity).

MI-EPI: To obtain a $\mathrm{T}_{1}$-anatomy identically distorted as the functional images (see below), an MI-EPI dataset was acquired at $0.7 \mathrm{~mm}$ isotropic (60 oblique coronal slices; GRAPPA $=3$; partial-Fourier $_{\text {phase }}=6 / 8$; matrix $=214 \times 214$; fatsaturation; $\mathrm{BW}=1016 \mathrm{~Hz} / \mathrm{px}$; Ref. lines $\mathrm{PE}=63$; phase-encoding $=\mathrm{R} \gg \mathrm{L}) .30$ inversion times (TI) were acquired with $2 \mathrm{D}$-EPI readouts $\left(\mathrm{TI}_{1}=175 \mathrm{~ms}\right.$; scan time per slice $=81.75 \mathrm{~ms} ;$ Excitation flip angle $=73^{\circ} ;$ echo-spacing $=1.09 \mathrm{~ms} ;$ EPI factor $=214 ; \mathrm{TE}=29 \mathrm{~ms} ; \mathrm{TR}=5107.2 \mathrm{~ms} ;$ series-order $=$ ascending) following a $15 \mathrm{~ms}$ tr-FOCI inversion pulse optimised for an inversion thickness of $200 \mathrm{~mm}$ and the

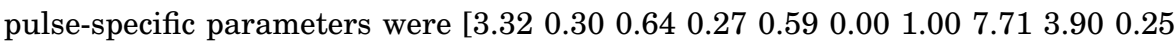
0.40] (see Table 1, Pulse 4 in Hurley et al. [139]). The slice ordering was changed 
every TR [125] by permuting a slab of slices (in our case, slab-size of 2) so that the slices have different inversion times than in the previous TR [131]. Therefore, each run lasted $154 \mathrm{~s}$ consisting of thirty volumes (one for each inversion time, see Fig. 2.2) and ten repetitions were acquired. An optimised 3*2 GRAPPA kernel [88] was used to reconstruct the images at all TIs. The reference lines were acquired without inversion preparation using FLEET [140].

\section{Functional data}

GE BOLD functional datasets were acquired for each participant at $0.7 \mathrm{~mm}$ isotropic resolution (52 oblique coronal slices; GRAPPA $=3$; flip angle $=16^{\circ}$; $\mathrm{BW}$

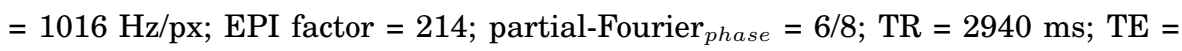
$26 \mathrm{~ms}$; phase-encoding $=\mathrm{R} \gg \mathrm{L}$; echo-spacing $=1.09 \mathrm{~ms}$ ) using a 3D-EPI readout [141]. Ten volumes with opposite phase-encoding but with otherwise identical imaging parameters, were acquired after each run to allow for distortioncorrection.

\subsubsection{Data processing and analysis}

The processing of the anatomical and functional data was performed using two distinct pipelines, namely, the Conventional and MI-EPI workflows, as illustrated in Figure 2.3 .

\section{Anatomical data}

Conventional workflow: $\mathrm{T}_{1}$-values and GM-WM contrast derived using the MP2RAGE sequence can be affected by $\mathrm{B}_{1}^{+}$transmit inhomogeneities [138]. Therefore, the quantitative $\mathrm{T}_{1}$ image of the MP2RAGE was corrected using the Sa2RAGE 


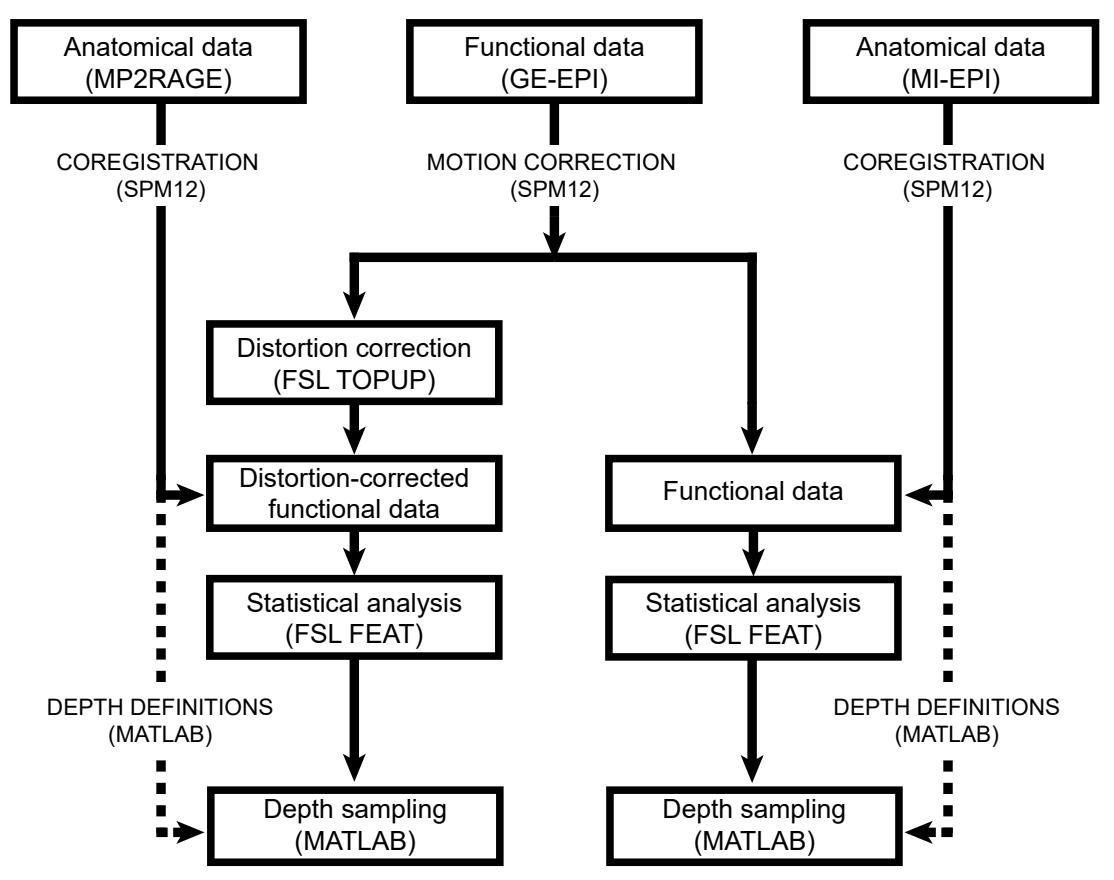

Figure 2.3: Data processing and analysis steps in the Conventional workflow (left) and MI-EPI workflow (right). For details, please see the text.
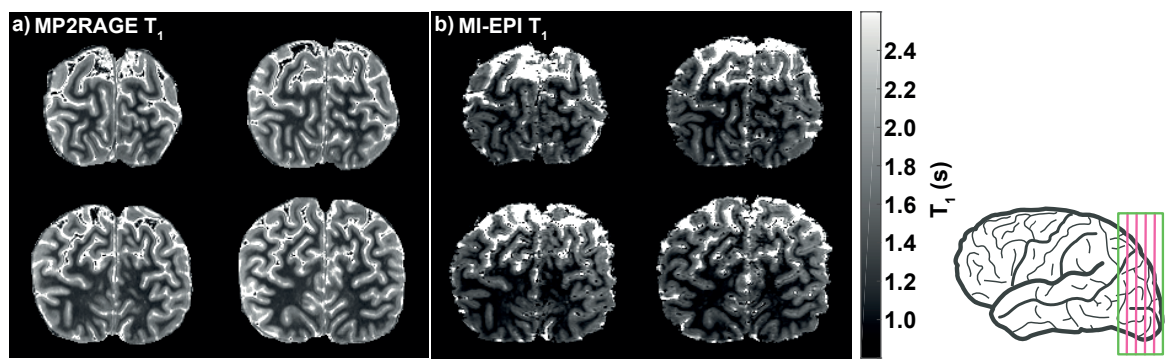

Figure 2.4: Four coronal slices from the quantitative $T_{1}$ maps obtained using a) Sa2RAGE-corrected MP2RAGE and b) MI-EPI, from a single participant (Note: The dark CSF voxels in the MP2RAGE are an artefact of the $B_{1}^{+}$correction). 
data (Figure 2.4a) and the corrected $\mathrm{T}_{1}$ map was computed in MATLAB [142]. The whole-brain MP2RAGE was cropped to a field-of-view (FOV) comparable to that of the functional data using the FSL [143] function fslroi and reoriented (from sagittal) to correspond with the functional data (coronal) using fslswapdim. The cropping is important as only the anatomical slices relevant for the functional data analysis are considered in the cost-function and the prior reorientation of the MP2RAGE to reduce large rotations during coregistration.

MI-EPI workflow: Ten repetitions of each inversion time of the MI-EPI were averaged and a quantitative $\mathrm{T}_{1}$ map (Figure 2.4b) was computed offline in MATLAB (MathWorks, USA). The non-linear least squares fitting algorithm based on an adapted version of the three-parameter inversion-recovery model (see Eq. 4 in Stikov et al. [144]), which did not require the exact knowledge of the voxel-specific flip angles, was used to compute the quantitative $\mathrm{T}_{1}$ values.

Segmentation: Initial segmentation was done on the co-registered $\mathrm{T}_{1}$ maps, independently for both the MI-EPI and MP2RAGE data using SPM12 [145, 146]. The tissue probability maps for GM, WM and CSF were then thresholded (>0.5) and binarised into tissue-specific masks. The accuracy of the segmented GM ribbon across all slices in the ROI is very critical for the laminar analysis. Therefore, the tissue-masks were manually corrected using ITK-SNAP v.3.4.0 [147] , in regions where the automatic segmentation was not accurately following the cortical ribbon (e.g., a missing voxel in an otherwise contiguous patch of GM), to obtain an optimal delineation of the GM. 


\section{Coregistration analysis}

To evaluate the coregistration discrepancy between the Conventional and MI-EPI workflows, we investigated the case where the MP2RAGE is geometrically distorted to closely match the functional data. If distortion-correction were to be optimal in un-distorting the functional data into anatomical (MP2RAGE) space, the inverse transformed MP2RAGE using the same voxel displacement estimates should also accurately conform to the native (distorted) EPI space. By sampling from the native EPI data, any discrepancies observed from this approach will most likely reflect errors in registration. The topup voxel shift estimated from the functional data was applied in the opposite direction using FSL's applytopup to the MP2RAGE $\mathrm{T}_{1}$ and its GM mask obtained from its automatic segmentation, thereby distorting them similarly to the mean EPI. From the MI-EPI, distortedMP2RAGE images and their segmentation masks, voxels were classified as either overlapping or non-overlapping voxels. An intersection operation of the MI-EPI and distorted-MP2RAGE segmentation masks was first carried out to identify the overlapping GM voxels (Figure 2.5b). An exclusive disjunction operation between the overlapping GM mask and the distorted-MP2RAGE GM segmentation was calculated to identify the non-overlapping distorted-MP2RAGE GM voxels (Figure 2.5c), i.e., voxels that are exclusively GM in the distorted-MP2RAGE mask and not in the MI-EPI mask. The same approach was used to identify the non-overlapping MI-EPI GM voxels (Figure 2.5d). Probability density histograms of the mean 3D-EPI signal across the non-overlapping voxels underneath the GM masks were computed for the MI-EPI and the distorted-MP2RAGE in a medial occipital ROI (Figure 2.5f, approximately V1) and in a lateral occipital ROI (Figure 2.5g, approximately V2/V3) which exhibited different extent of distortions along the phase-encoding direction. It is important to note that 


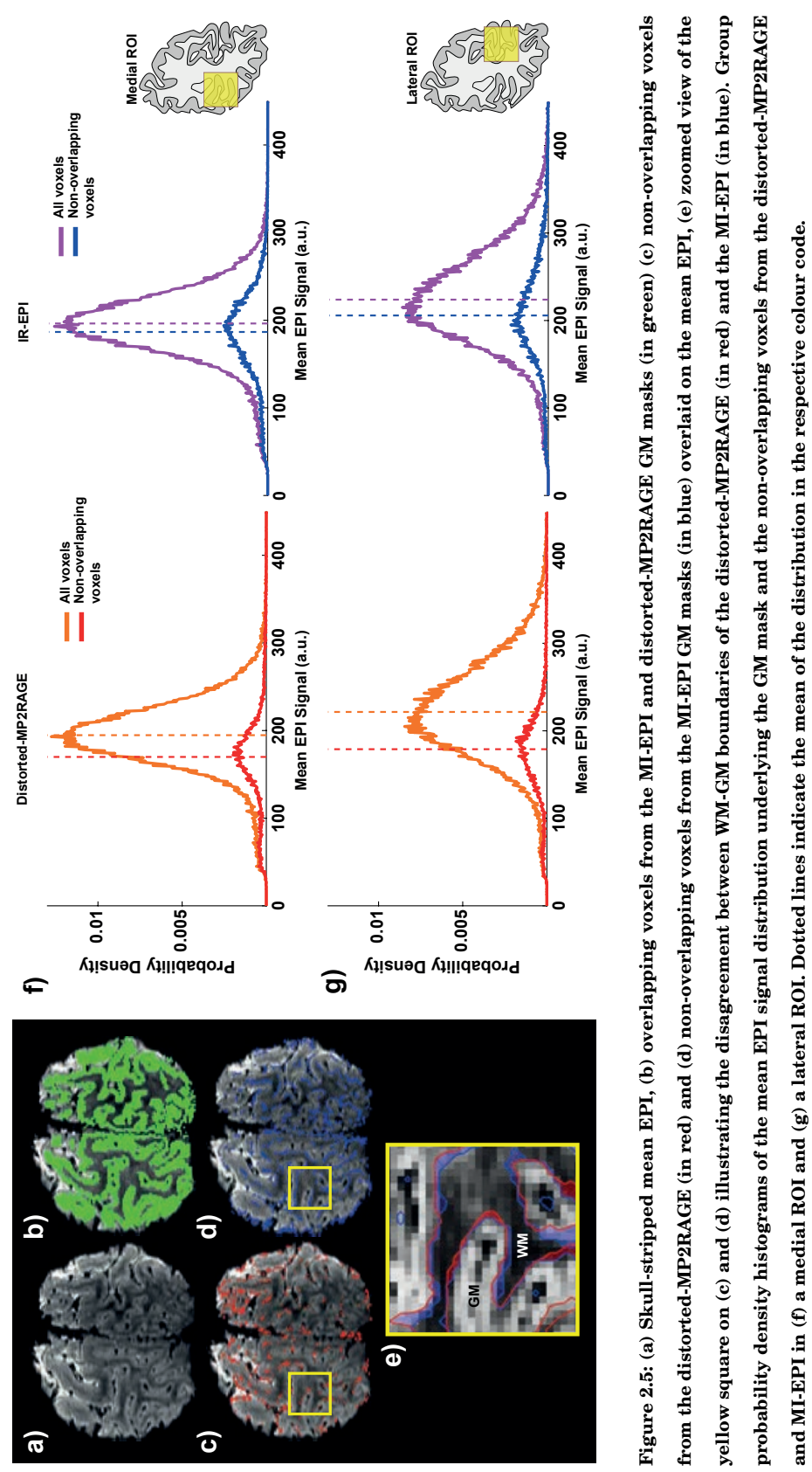


this step was solely performed to quantify the quality of the coregistration and was not further used to analyse the functional data in either of the two workflows.

\section{Functional data}

The functional runs were motion-corrected using SPM12 and no spatial smoothing was applied. Following this, the functional datasets were analysed in two independent workflows (Fig. 2.3) based on whether distortion-correction was applied (Conventional workflow) or not (MI-EPI workflow). All functional datasets were high-pass filtered in FSL using a Gaussian-weighted least-squares fitting $(\sigma=60 \mathrm{~s})$. Statistical analysis of the time-series was carried out in FSL using FEAT v.6.0 (FMRI Expert Analysis Tool) using FILM with local autocorrelation correction [148]. The time-series GLM model included a double gamma HRF with temporal derivatives and motion parameters as nuisance regressors. The withinparticipant higher-level analysis was carried out using a fixed effects model by forcing the random effects variance to zero in FLAME (FMRIB's Local Analysis of Mixed Effects) [149, 150]. The z-scores were computed in each workflow separately for static and flicker conditions without spatial smoothing.

Conventional workflow: The functional runs were distortion-corrected with opposite phase-encoding images using a custom configuration file that was optimised for the high-resolution data using FSL's topup [121, 143, 151]. The $\mathrm{T}_{1}$ image from the MP2RAGE was then co-registered to the mean distortion-corrected functional image using SPM12. The statistical analysis using FSL FEAT was then carried out on the distortion-corrected functional datasets as described above.

MI-EPI workflow: The anatomical $\mathrm{T}_{1}$ map calculated from the MI-EPI data was 
co-registered to the mean functional image using SPM12 [152]. Note that, in contrast to several high-resolution fMRI studies (e.g. [66, 77, 94, 98, 153]), we coregister the anatomical image to the functional data [68] to minimise smoothing and interpolation of the functional data.

Regions-of-interest (ROI): The large values in the statistical maps are usually due to draining veins near the activated region [154-156]. These voxels were excluded from the analysis by thresholding the z-scores from the second-level FEAT analysis for each condition between $50-90 \%$ of the maximum z-score because the highest z-scores are very likely to arise from the large signal amplitude in pial vessels [66] or voxels with large contribution of ascending veins and, thereby, the procedure improved the spatial specificity of the activation clusters by removing remaining vein-dominated voxels. An intersection of these thresholded z-maps from the flicker and static conditions was used to obtain clusters of voxels that were activated in both stimulus conditions. The two largest contiguous clusters were selected per participant yielding a total of ten ROIs in each analysis workflow. The statistical activation clusters were masked with the segmented GM ribbon from the anatomical data.

\section{Laminar analysis}

Definition of laminae: The manually corrected ROI segmentations from ITKSNAP were exported as 3D surfaces for GM, WM and CSF. For each ROI, the three tissue surfaces were imported into MATLAB and were used to define the GM-CSF and WM-GM boundaries at five times the voxel resolution of the acquired data. Ten laminar surfaces were created including the GM-CSF and WM-GM 
boundaries using the equi-volume principle $[70,71]$ resulting in nine laminae in GM (Fig. 2.6). Since a voxel in the acquired spatial resolution can lie across several cortical depths, the high-resolution equi-volume laminar surfaces generated were used to compute a 3D partial-volume matrix of the depth-dependence for every voxel's signal in the resampled ROI.

Laminar sampling: The voxels from the functional data for all ROIs were resampled using nearest-neighbor interpolation to five times the acquired resolution and assigned to the different laminae in a winner-takesit-all approach using the 3D laminar partial-volume matrix i.e., voxels with the highest weighting to a specific layer in the 3D laminar partial-volume matrix are assigned to that particular lamina and the signal time-courses were then

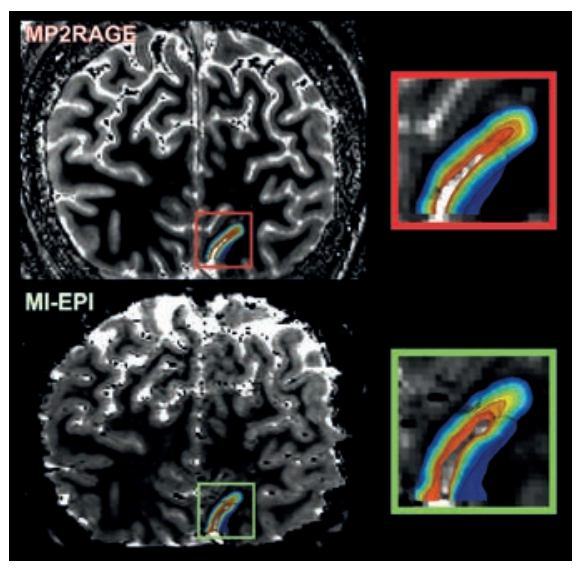

Figure 2.6: Example laminar surfaces generated in a manually segmented ROI from MP2RAGE $T_{1}$ (top) and MI-EPI $T_{1}$ (bottom) and zoomed in view of the laminar surfaces are presented as insets. averaged separately for each lamina.

Laminar profile calculation: To examine the different components of the eventrelated time-courses, we computed the laminar profiles by averaging the signal amplitudes between 6-18 s following the stimulus onset, i.e. PB signal, and 27-39 s, i.e. PSU, for each lamina. 


\section{Laminar profile normalisation}

The two main vascular biases in GE fMRI, (a) draining of deoxy-Hb, $d H b$ for short, from lower to upper layers of the cortex via ascending veins and (b) baseline CBV and relaxation parameters such as $\mathrm{T}_{2}{ }^{*}$, can be different for different layers. To examine if the laminar neuronal specificity of the signal can be recovered from the data, we used a normalisation approach. Normalisation of laminar profiles have been proposed previously in the context of resting-state fMRI $[157,158]$ using BOLD fluctuation power to probe connectivity across areas and with induced hypercapnia [158]. These normalisation approaches are motivated by the fact that the baseline parameters, such as venous $\mathrm{CBV}\left(\mathrm{V}_{0}\right)$, are assumed to be multiplicative for BOLD sensitivity and conforms to the single-vascular-compartment BOLD signal models (as in Davis et al. [159], Buxton et al. [160]). Thus, under this assumption, the $\mathrm{V}_{0}$ bias can be accounted for by the division of laminar signal amplitudes elicited for the two different stimuli or across time-points. We can describe this by formulating the BOLD signal change, $\Delta \mathrm{S}$, as a product of two functions (Eq. 2.1).

$$
\Delta S=f\left(V_{0}, E_{0}, T_{2}^{*}, T E, \ldots\right) \cdot g(\Delta V, \Delta d H b)
$$

where the term $f\left(V_{0}, E_{0}, T_{2}^{*}, T E, \ldots\right)$ is a function of the baseline physiological and physical parameters influencing the BOLD signal, i.e. baseline blood volume, $\mathrm{V}_{0}$, baseline oxygen extraction fraction, $\mathrm{E}_{0}$, baseline transverse relaxation time constant in GE measurements, $\mathrm{T}_{2}{ }^{*}$, and echo-time, $\mathrm{TE}$ and the term $g(\Delta V, \Delta d H b)$ is a function of the change in physiological parameters due to activation, i.e. changes in $\mathrm{CBV}, \Delta \mathrm{V}$, and changes in deoxy-Hb, $\Delta \mathrm{dHb}$. In the context of laminar fMRI studies, $g(\Delta V, \Delta d H b)$ consists approximately of two additive components stemming from local micro-vasculature and the draining ascending veins [43]. Both 
the Davis [159] and Buxton [160] models can be formalised in this way. This is a one vascular-compartment model, which is well justified for the strong venous weighting of the GE fMRI signal [43]. For a given layer, $L=f\left(V_{0}, E_{0}, T_{2}^{*}, T E, \ldots\right)$ is constant. Typically, for low- and high-resolution fMRI studies, two stimulus conditions are contrasted by a subtraction operation (Eq. 2.2).

$$
\Delta S_{\text {flicker }}-\Delta S_{\text {static }}=L \cdot\left(g_{\text {flicker }}-g_{\text {static }}\right)
$$

Note that the difference of the signal changes is still dependent on $L$. Since $L$ is not independently measured, the laminar profile of the difference between conditions is weighted by the baseline physiology and relaxation parameters of the different layers. For a given layer, $L$ is constant for the two stimulus conditions and/or timepoints. Therefore, a division (rather than a subtraction) can eliminate the effect of $L$ and would be the more appropriate way to account for the baseline CBV (Eq. 2.3).

$$
\frac{\Delta S_{\text {flicker }}}{\Delta S_{\text {static }}}=\frac{g_{\text {flicker }}}{g_{\text {static }}}
$$

Thus, calibrating the BOLD signal to another condition or time point (or baseline fluctuations $[157,158])$ effectively removes the bias stemming from baseline physiological and physical parameters. For the case that neuronal profiles of two stimuli or time-points are just scaled versions of each other (i.e. $g_{\text {flicker }}=s c \cdot g_{\text {static }}$ ) and assuming that the function $g$ is linear for a range of changes in CBV and deoxy- $\mathrm{Hb}$ (which is justified for the signal range in the tissue). That is, if two stimuli or time-points evoke the same laminar neuronal profile with some scaling factor, $s c$, then the division approach yields a constant value for all layers. In other words, a deviation from a constant profile after division is an indicator of laminar differences in neuronal activity. For example, if the division approach yields a 
laminar profile with constant values up to a specific layer, then the neuronal activities for all layers below this layer are similarly scaled when comparing both conditions/time-points. Therefore, the division approach can, on the one hand, be used to remove baseline parameter biases and on the other hand, be utilised as a tool to explore and characterise the cortical depth profiles by taking the ratio of the laminar profiles between the two stimulus conditions (in this case, Flicker/Static) or between two different portions of the time-course (in this case, PSU/PB) [153, 161]. Note that in our study, this approach yields (see below), results comparable to animal studies: it has been shown $[132,162]$ that the PSU is better spatially localised in GM relative to the $\mathrm{PB}$ response.

\section{Venous and CSF time-courses}

In low resolution fMRI studies, the most dominant source of the fMRI signal contrast stems from cortical surface veins located in the CSF. In order to study differences of the time-courses, the CSF and dominantly venous voxels were manually selected for all ROIs. The venous voxels were identified with the following approach: (a) the temporal mean of the functional EPI data was computed using fslmaths, voxels with low intensity were selected from the mean EPI image by setting an arbitrary threshold (in this case, $<60$ mean EPI signal units) so as to not include any GM voxels partial volume with a neighboring low intensity voxel, (b) the voxels with low tSNR were selected by setting a threshold (in this case, $<8$ ) that also excludes most GM voxels and the combined mask of venous voxels was computed by a union operation of the low intensity (a) and low tSNR (b) maps (Fig. 2.7). CSF voxels were selected from the tissue class segmentation of the $\mathrm{T}_{1}$ map for every ROI. Since venous voxels can be contaminated by $\mathrm{CSF}$, the CSF voxels were selected as a subset of the CSF segmentation mask 
that does not overlap with the venous voxels. Event-related average time-courses were calculated from these venous and CSF voxels and compared with the midGM time-course from the laminar analysis. To better visualise the differences, the three time-courses were normalised to the time-point corresponding to the end of the stimulus.
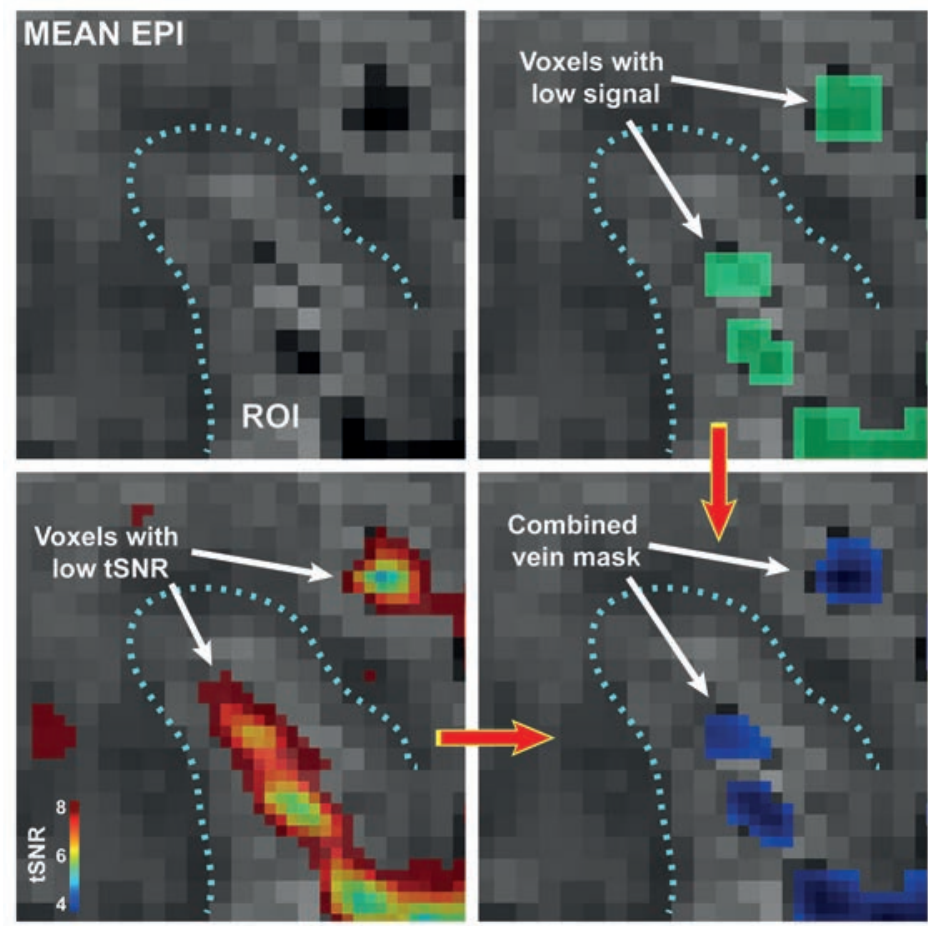

Figure 2.7: (top-left) Zoomed in view of a single slice of the mean EPI showing an ROI (dotted lines), (top-right) map of voxels with low mean EPI signal overlaid, (bottom-left) voxels with low tSNR overlaid on the mean EPI, (bottom-right) combined vein mask of voxels with both low mean EPI signal and low tSNR. 


\section{Relative ROI size analysis}

The size of the ROI and therefore, the number of voxels averaged is usually determined by the activation map in the region. Averaging laminar signals across all the activated voxels in a relatively large ROI may affect the magnitude of the effect that one observes and this magnitude may differ between the two workflows. Note that, for studies probing higher-order cognitive processing, the activation ROI is usually much smaller in size than for the checkerboard stimuli utilised in this study. Therefore, the dependency of the size of the ROI on the magnitude of the differences between the two workflows was explored. The size of an ROI was calculated as the tangential distance along the GM for every slice at every cortical depth. The center or seed for the ROI was determined by the voxel showing the largest condition difference in GM after computing a contrast between flicker and static. Note that this does not imply largest differences between the workflows. This analysis was also repeated using randomly selected seed voxels in a ROI. Ten percent of the total length of the ROI ( $\approx 60$ voxels on average) was taken as the minimum ROI size to have enough number of voxels to obtain sufficient SNR. This ROI was grown tangentially in both directions by five percent on each side to obtain the next ROI, i.e. twenty percent total length. The GM ROI was thus grown tangentially in increments of ten percent till ninety percent of the total length was reached. In the case, wherein the edge of the ROI was reached in one direction, the ROI was grown by ten percent only in the other direction. Five percent on either end of the ROI was not included in this analysis to avoid any edge errors of the segmented ROI. The $\mathrm{z}$-statistic maps were thresholded using $\mathrm{z}>2.3$ and a corrected cluster significance threshold of $p=0.05$, which is the default option in FSL FEAT. This threshold was considerably more liberal than the one used as the activation mask in the laminar analyses and yielded larger number of voxels per ROI, 
allowing us to grow the ROIs within a relatively larger patch of cortex while still be within the activation mask. The laminar profiles for the PB signal were calculated for each relative ROI size in both workflows. We calculated the magnitude of the laminar signal differences between the workflows (MI-EPI - Conventional) for the two stimulus conditions across different ROI sizes. The magnitude of the laminar signal differences was then summed across depths and averaged across participants to obtain the total difference in signal for every ROI size. Contrary to computing differences from the mean signal in the ROI, this approach provides a measure of the laminar signal differences.

\subsection{Results}

\subsubsection{Anatomical data}

\section{Quantitative $\mathbf{T}_{1}$ comparison}

Four coronal slices from the Sa2RAGE-corrected MP2RAGE $\mathrm{T}_{1}$ map on a representative participant is shown next to the corresponding MI-EPI $\mathrm{T}_{1}$ map (Fig. 2.4) to illustrate the quality of both anatomical datasets. The GM and WM quantitative $\mathrm{T}_{1}$ estimates in the occipital lobe for all participants are listed in Table 2.1. The average $\mathrm{T}_{1}$ estimate was calculated across four out of five participants in whom Sa2RAGE was acquired. The WM and GM $\mathrm{T}_{1}$ estimates were shorter in MI-EPI when compared to the MP2RAGE (mean difference $=0.215 \pm 0.078 \mathrm{~s}, \mathrm{p}=0.012$ and $0.202 \pm 0.157 \mathrm{~s}, \mathrm{p}=0.082$, respectively). All $\mathrm{p}$-values reported are adjusted for multiple comparisons using Bonferroni correction. 
Table 2.1: Quantitative $T_{1}$ values of grey and white matter estimated with MP2RAGE and MI-EPI for all participants. Note that $T_{1}$ average was computed from participants 1-4 because the Sa2RAGE could not be acquired in participant 5.

\begin{tabular}{ccccc}
\hline \multirow{2}{*}{ Participant } & \multicolumn{2}{c}{ GM T $\mathbf{T}_{1}(\mathbf{s})$} & \multicolumn{2}{c}{ WM T } \\
\cline { 2 - 5 }$(\mathbf{s})$ \\
\cline { 2 - 5 } & MP2RAGE & MI-EPI & MP2RAGE & MI-EPI \\
\hline 1 & $1.79 \pm 0.27$ & $1.37 \pm 0.19$ & $1.26 \pm 0.11$ & $0.94 \pm 0.13$ \\
2 & $1.62 \pm 0.15$ & $1.56 \pm 0.25$ & $1.19 \pm 0.09$ & $1.04 \pm 0.14$ \\
3 & $1.64 \pm 0.25$ & $1.52 \pm 0.31$ & $1.16 \pm 0.09$ & $0.93 \pm 0.19$ \\
4 & $1.60 \pm 0.21$ & $1.39 \pm 0.24$ & $1.12 \pm 0.07$ & $0.96 \pm 0.14$ \\
5 & $1.90 \pm 0.19$ & $1.46 \pm 0.23$ & $1.32 \pm 0.11$ & $0.98 \pm 0.15$ \\
\hline Average & $\mathbf{1 . 6 6} \pm \mathbf{0 . 2 2}$ & $\mathbf{1 . 4 6} \pm \mathbf{0 . 2 5}$ & $\mathbf{1 . 1 8} \pm \mathbf{0 . 0 9}$ & $\mathbf{0 . 9 7} \pm \mathbf{0 . 1 5}$ \\
\hline
\end{tabular}

\section{Co-registration analysis}

In Figure 2.5, we show the probability density histogram for all voxels within the MI-EPI and the distorted-MP2RAGE GM masks in the medial ROI (Fig. 2.5f), indicating the mean EPI signal values of $196.30 \pm 10.59$ and $194.706 \pm 9.88$, respectively. For the lateral ROI (Fig. 2.5g), the mean EPI signals of all GM voxels in MI-EPI and distorted-MP2RAGE maps were $223.85 \pm 19.97$ and $221.55 \pm 22.64$, respectively. In both the medial and lateral ROIs, the mean signal from the nonoverlapping voxels of the distorted-MP2RAGE was significantly lower compared to the mean GM EPI signal (mean difference for medial ROI: $26.42 \pm 5.32$, p < 0.001 , and for lateral ROI: $44.87 \pm 9.9, \mathrm{p}=0.001$ ). However, no significant difference between the mean values for the non-overlapping voxels was found for MIEPI, indicating higher accuracy of the registration as compared to the MP2RAGE workflow. There was no statistically significant difference $(t(8)=1.424, p=0.1922)$ in the number of GM voxels across participants between the MI-EPI (16090 \pm 844 


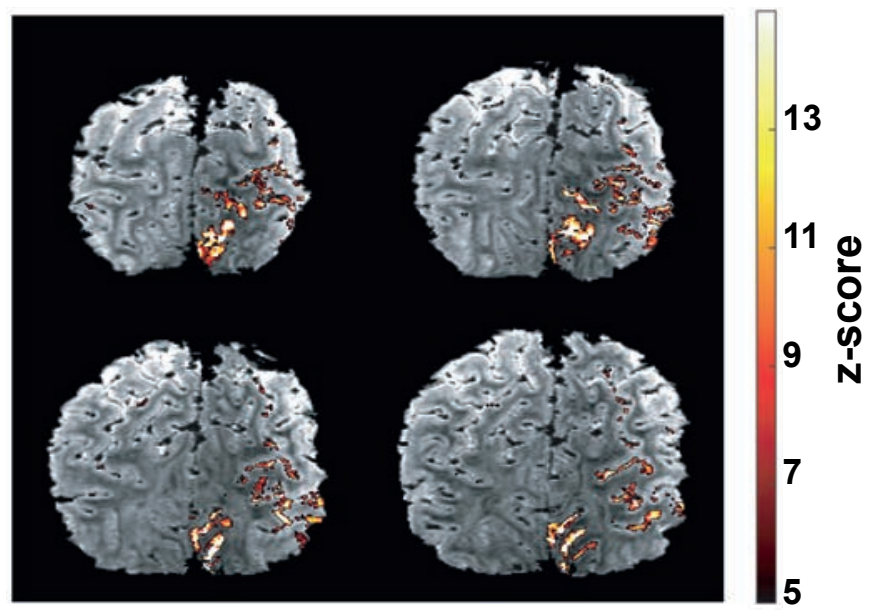

Figure 2.8: Activation map of a single subject for the flickering stimulus overlaid on four slices of the mean EPI from the MI-EPI workflow.

voxels) and the distorted-MP2RAGE (14499 \pm 731 voxels) ROIs used in this analysis.

\subsubsection{Functional data}

The activation map from a single participant for the flicker condition from the MIEPI workflow is shown in Figure 2.8. The static condition also activated the same spatial subset of voxels as the flicker condition. An intersection of the thresholded z-statistic maps of flicker and static conditions in the ROIs was used for the subsequent laminar analyses yielding an average of $160 \pm 19$ GM voxels (in the acquired resolution) per ROI. Robust activation clusters were detected in both ROIs and stimulus conditions for all participants. 
a) Conventional Workflow

FLICKER

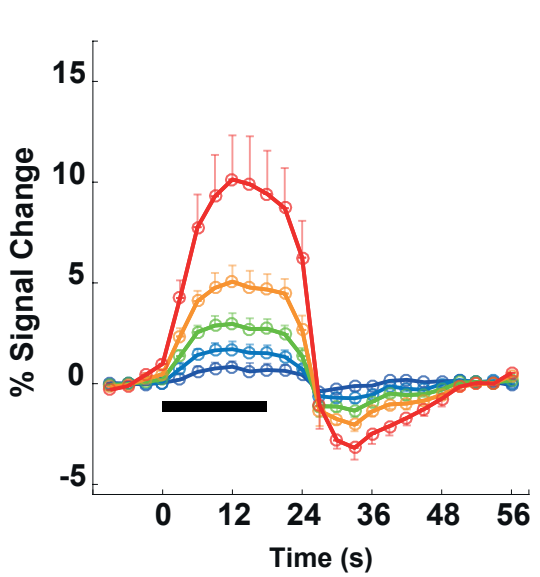

STATIC

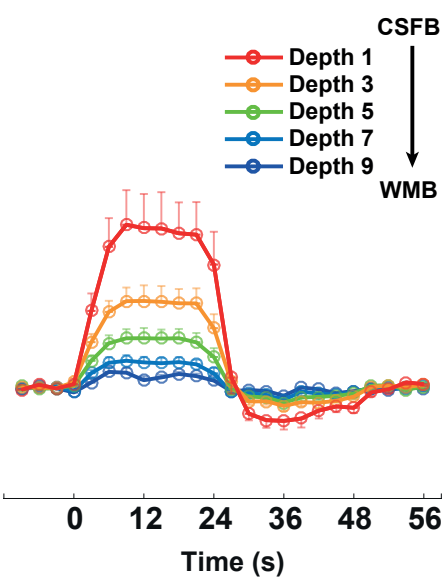

b) MI-EPI Workflow

FLICKER

STATIC

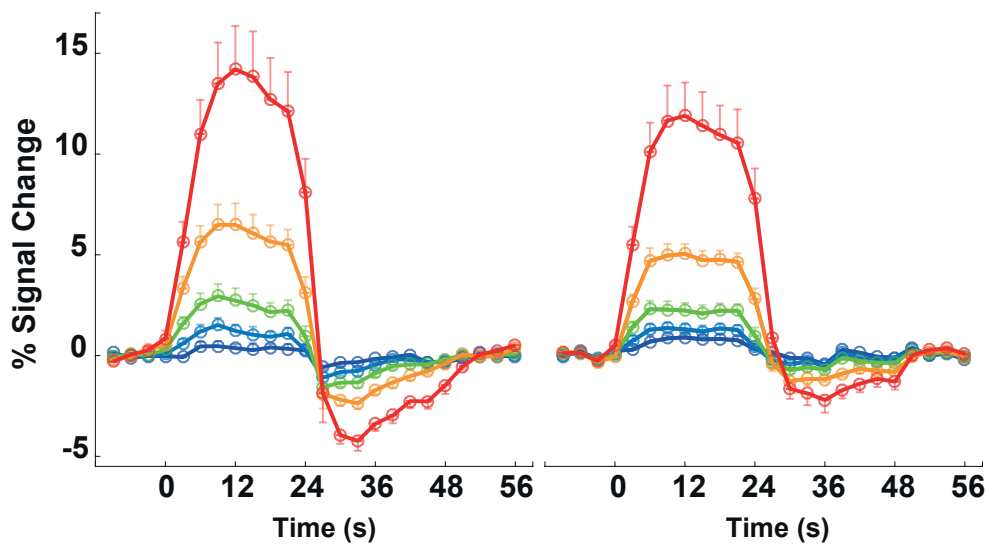

Figure 2.9: Laminar time-courses (five out of nine GM depths shown) for the two stimulus conditions in the (a) Conventional and (b) MI-EPI workflows, respectively (horizontal black bar indicates stimulus duration). Error bars indicate SEM. 
POSITIVE BOLD

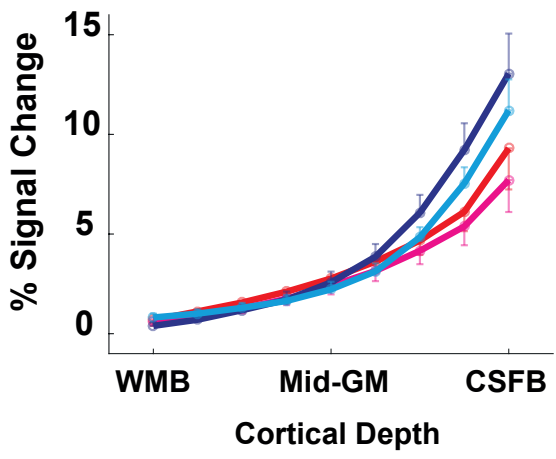

POST-STIMULUS UNDERSHOOT

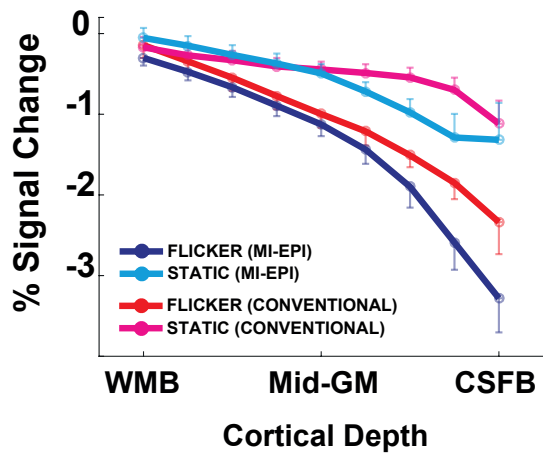

Figure 2.10: Laminar profiles for both conditions in two workflows for the Positive BOLD (left) and PostStimulus Undershoot (right). Error bars indicate SEM. CSFB - CSF boundary, WMB - WM boundary.

\section{Laminar analysis}

Event-related average time-courses computed for both stimulus conditions in the two workflows are shown in Figure 2.9. The dynamic range of the signal amplitude of laminar time-courses is higher in the MI-EPI workflow (Fig. 2.9b) compared to the Conventional workflow (Fig. 2.9a) for both stimulus conditions. The flicker condition elicited a much stronger PSU than the static condition and is visible in both workflows. In both workflows, the laminar profiles for the PB signal (Fig. 2.10, left) show a signal increase towards the more superficial layers (towards the GM-CSF boundary) in both stimulus conditions, typical for GE fMRI acquisitions. The PB profile from the MI-EPI workflow differs significantly from the Conventional workflow in the two uppermost layers in both stimulus conditions (flicker: $\mathrm{F}_{(1,81)}=4.946, \mathrm{p}=0.0289$, mean-difference depth $1=3.746 \% \mathrm{BOLD}, \mathrm{p}$ $=0.0038$, mean-difference depth $2=3.108 \% \mathrm{BOLD}, \mathrm{p}=0.0279 ;$ static: $\mathrm{F}_{(1,81)}=7.458$, $\mathrm{p}=0.0077$, mean-difference depth $1=3.494 \% \mathrm{BOLD}, \mathrm{p}=0.0002)$. In the case of the 

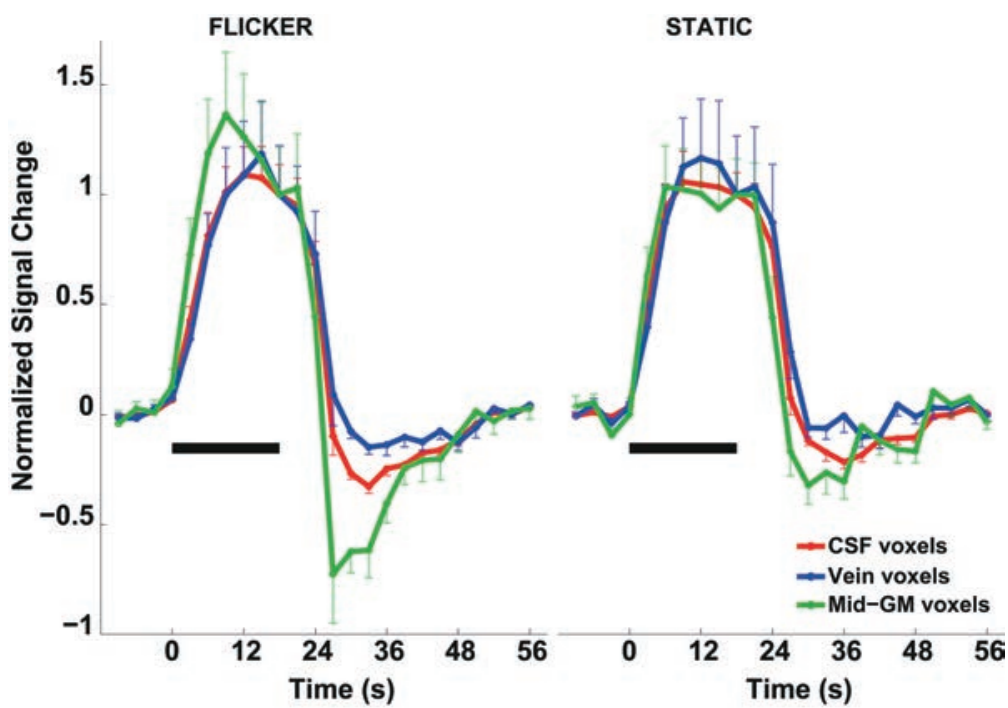

Figure 2.11: Normalised event-related time-courses from voxels in the CSF and veins in the ROIs compared to the mid-GM time-course for the flicker (left) and static (right) conditions from the MI-EPI workflow. Error bars indicate SEM.

PSU for the flicker condition (Fig. 2.10, right), a trend similar to the PB signal is observed with significant differences between workflows being at the most superficial layers $\left(\mathrm{F}_{(1,81)}=11.98, \mathrm{p}=0.0009\right.$, mean-difference depth $1=-0.9436 \% \mathrm{BOLD}$, $\mathrm{p}=0.0122$ ). In the static condition, however, the differences in the PSU between the two workflows was most significant at the two cortical depths immediately below the most superficial layer $\left(\mathrm{F}_{(1,81)}=5.205, \mathrm{p}=0.0251\right.$, mean-difference depth $2=$ $-0.5899 \%$ BOLD, $\mathrm{p}=0.0074)$.

\section{GM, vein and CSF time-courses}

Figure 2.11 shows the time-courses for mid-GM, venous and CSF voxels for both stimulus conditions. The differences in the BOLD times-to-peak are not statisti- 
cally significant $\left(\mathrm{F}_{(2,27)}=1.663, \mathrm{p}=0.208\right)$. We found a statistically significant difference in the magnitude of PSU between the three time-courses $\left(\mathrm{F}_{(1.02,6.117}\right)$ $=10.29, \mathrm{p}=0.017)$, with a stronger undershoot in the mid-GM compared to the CSF (mean-difference $=-0.2174 \pm 0.0851 \% \mathrm{BOLD}, \mathrm{p}=0.129$ ) and a significantly stronger mid-GM undershoot compared to the pial veins (mean-difference $=-0.3504$ $\pm 0.1028 \% \mathrm{BOLD}, \mathrm{p}=0.043)$. Similar time-course characteristics are present also during the static condition, albeit with a much smaller undershoot response.

\section{Relative ROI size analysis}

Figure 2.12 shows the effect of increasing ROI size on the sum of the absolute differences of the laminar PB signal between the MI-EPI and Conventional workflows for the two stimulus conditions. In the results presented earlier, the average ROI size was about $26 \%$ with respect to the scale shown in Figure 2.12 $(\approx 160$ voxels $)$. A decrease in the total difference between the two workflows is observed with increasing ROI size for both stimulus conditions (Fig. 2.12). Despite averaging over the largest ROI size, there is still a $4.52 \%$ BOLD (flicker) and $3.16 \%$ BOLD (static) difference between the two workflows, which is about $13.57 \%$ (flicker) and $10.68 \%$ (static) of the total laminar PB signal in the ROI.

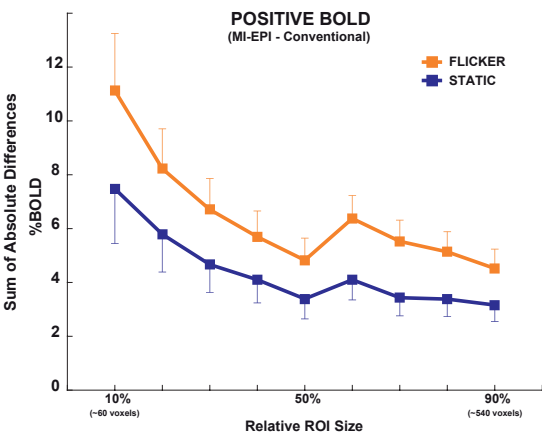

Figure 2.12: The sum of absolute differences in the laminar BOLD signal between the workflows (MI-EPI minus Conventional) is shown with respect to increasing ROI size for the two stimulus conditions. Error bars indicate sum of squared errors. 


\section{Laminar profile normalisation}

Due to the fact that strong differences in the deepest layers can be driven by division of numerically small BOLD signal changes, we restricted our ANOVA analysis to seven of the nine layers, excluding the deepest two. In the first normalisation (Fig. 2.13a), the ratio of the PB depth profiles of the flicker relative to the static response in the Conventional workflow does not reveal any significant depth-specific modulation $\left(\mathrm{F}_{(6,63)}=0.208, \mathrm{p}=0.97\right)$. However, in the MI-EPI workflow, we observe that the $\mathrm{PB}$ is proportionally larger in amplitude in the flicker compared to the static condition towards the pial surface and this effect reverses with increasing depth $\left(\mathrm{F}_{(6,63)}=2.154, \mathrm{p}=0.059\right)$. The ratio of the laminar profiles obtained using different time-segments of the haemodynamic response, i.e. PSU to the PB ( 2.13b), shows no significant differences between MI-EPI and Conventional workflows for the static condition. However, the two workflows exhibit contrasting behaviour for the flicker condition. In the MI-EPI workflow, the PSU increases

a)

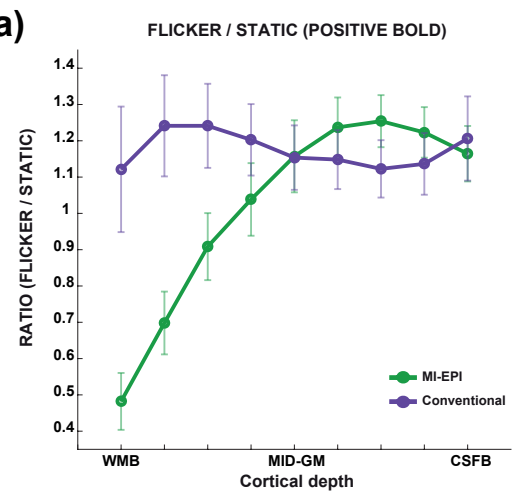

b)

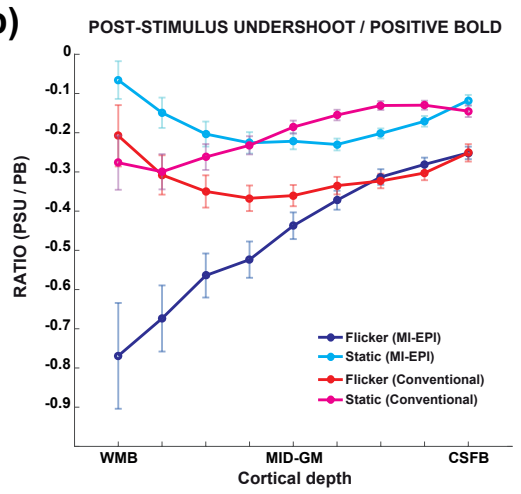

Figure 2.13: a) Ratio of the laminar PB profiles of the two stimulus conditions (flicker to static) from the MI-EPI and Conventional workflows and b) the ratio of different time-segments (PSU to PB) for the two workflows and stimulus conditions. 
proportionally to the PB signal with increasing depth whereas the Conventional workflow shows the opposite pattern.

\subsection{Discussion}

In the present study, we compared the implications of the data acquisition strategy on the subsequent processing and analysis of laminar fMRI data (Fig. 2.3) and, additionally, presented an approach to reduce vascular bias in GE BOLD fMRI.

\subsubsection{Anatomical data}

The conventional data acquisition strategy involves differently-distorted anatomical and functional data (in this case, MP2RAGE \& EPI). We propose acquiring high-resolution anatomical data using MI-EPI instead. This approach has the advantage of being distortion-matched to the functional data. As a side result, the present study was well-placed to compare the $\mathrm{T}_{1}$ values over the occipital cortex (see Table 2.1). We observed shorter tissue $\mathrm{T}_{1}$ estimates with the MI-EPI than with the Sa2RAGE-corrected MP2RAGE. This could be, on the one hand, due to magnetization transfer (MT) effects from the fat saturation pulse used in MI-EPI, but not in the MP2RAGE [163]. In addition to MT-effects, imperfections in the excitation slice profiles could also cause partial saturation of the consecutive slice and, therefore, a potential bias in the $\mathrm{T}_{1}$ estimates. On the other hand, the estimation of $\mathrm{T}_{1}$ in MP2RAGE on just two inversion times may also be prone to estimation errors. Clearly, more work has to be done to determine the most reliable approach for quantitative $\mathrm{T}_{1}$ estimation, which, however, is beyond the scope of the current study. Although quantitative $\mathrm{T}_{1}$ determination may be a concern 
for myelin mapping studies, for the purposes of yielding sufficiently high tissue contrast for segmentation and delineation of laminae, the MI-EPI $\mathrm{T}_{1}$ map is comparable to the Sa2RAGE-corrected MP2RAGE T 1 map (Fig. 2.4, Fig. 2.6). Recently, MI-EPI has been shown to be of sufficient quality to perform automated whole-brain segmentation and surface-based analysis [131]. Lastly, the GRAPPA kernel used to reconstruct the multiple TI contrasts can introduce mild ghosting, therefore, the reconstruction must be optimised (as done in the present study) and each MI-EPI dataset must be carefully examined for the presence of such artefacts especially around the ROI.

\section{Co-registration quality}

The conventional acquisition strategy, utilised in low-resolution fMRI studies in particular, requires distortion-correcting the functional data using post-processing tools such as FSL's topup [121, 151], a preferred approach owing to some of the practical difficulties of the fieldmap-based distortion-correction [151, 164]. In

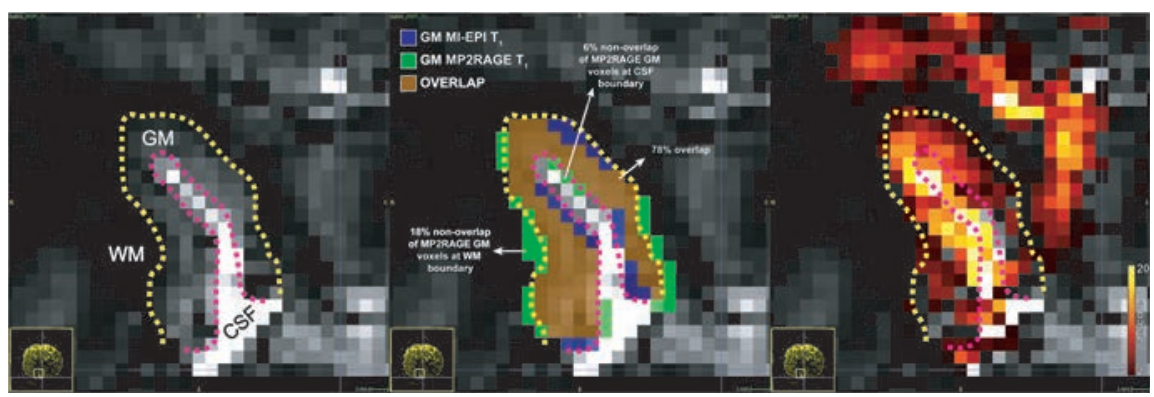

Figure 2.14: Left-to-right: Zoomed-in view of an ROI on the MI-EPI $T_{1}$ map of an example participant indicating the GM-WM and CSF-GM boundaries, same slice with overlay of the GM ROIs derived from MP2RAGE and MI-EPI $T_{1}$ and the ROI with an overlay of the activation map (z-statistic) for the flicker condition. The percentage overlap and non-overlap presented in the figure is the average across slices. 
distortion-correction approaches, the increased sensitivity to field inhomogeneities and sub-voxel subject motion limit the accuracy of estimating and correcting geometric distortions. Therefore, sub-optimal distortion-correction can result in coregistration errors between the functional and anatomical data. We demonstrate the use of a histogram-based approach to quantify registration quality between anatomical and functional data. By distorting the MP2RAGE to the EPI space, we could quantitatively compare the registration of the mean EPI image with the MI-EPI $\mathrm{T}_{1}$ and the distorted-MP2RAGE. Note that we registered only partial FOV centered on the ROIs. Full FOV registration is not advised in high-resolution fMRI studies as the cost function used by the registration algorithm takes irrelevant ROIs into account and thereby, increase registration errors on the relevant ROIs. The non-overlapping voxels were of interest in this analysis because they exclusively fall under the distorted-MP2RAGE or the MI-EPI GM mask (Fig. 2.5a-d, Fig. 2.14). The probability density histograms in the distorted-MP2RAGE shows that the non-overlapping voxels have significantly lower intensity than the mean GM signal. As we calculated the histograms from the same data (mean EPI in the native space), blurring due to sequence differences does not play a major role but rather the differences in the histograms indicate differences in the GM definition. Therefore, the shift of the peak (dashed lines) to the left (more WM contamination, lower signal) is an indicator of misregistration. Therefore, the shift of the peak (dashed lines) to the left (more WM contamination, lower signal) is an indicator of misregistration. In the MI-EPI workflow, the histogram of the non-overlapping and overlapping voxels from the GM mask are not significantly different indicating smaller degree of sampling CSF/WM signal and thus, demonstrates a high accuracy of registration. The effects of partial voluming with WM and CSF are region-dependent, the higher the underlying distortions are, the more likely it is 
that the GM fMRI signals are contaminated with those of WM and CSF, as illustrated in Figures 2.14 and 2.5f-g.

\subsubsection{Functional data}

We successfully replicated an earlier study [134] at $7 \mathrm{~T}$ using similarly designed stimuli and show that it is consistent across both workflows. The goal of the stimulus was to evoke robust activation with sufficient SNR at this sub-millimetre spatial resolution and not to preferentially activate a certain visual area. The functional ROI clusters for all subjects were approximately located in V1 and V2, and the stimulus-evoked responses show robust behaviour across depths. However, as the goal of this study was to compare different processing and acquisition approaches for laminar fMRI, the conclusions drawn from this study are independent of the exact locus of the activation. The EPI readout was kept as short as possible to achieve our target resolution and coverage. Here, it was on the order of twice the grey matter's $\mathrm{T}_{2}{ }^{*}$ through the use of in-plane acceleration. It is important to keep in mind that longer readout trains may result in increased $\mathrm{T}_{2}{ }^{*}$ blurring [57, 165], which may affect the laminar signal of interest. Both conventional and MI-EPI workflows resulted in robust activation maps for both stimulus conditions. The activation clusters were similarly spatially localised in both workflows. In contrast to most low- and high-resolution fMRI studies, we co-register the anatomy to the mean functional image to minimise the post-processing and transformations done to the laminar functional data. That is, this approach may already reduce smoothing associated with both the distortion-correction and subsequent registration of the functional data. 


\section{Laminar analysis}

The cortical depth profiles exhibit signal increases towards the uppermost depths, typical for GE-EPI shown previously [49, 57, 66, 94, 111, 133, 166]. A similar trend was observed for the depth profiles of the PSU [49, 153, 167]. The dynamic range of the laminar responses is lower in the Conventional workflow compared to the MI-EPI workflow (Fig. 2.9). In addition, the Conventional approach also results in a higher BOLD signal being observed in the deeper layers. The laminar profiles exhibit the differences between the two workflows in a more pronounced manner (Fig. 2.10). For the PB response, significant differences between the workflows were found in the upper cortical depths (close to the CSF boundary) and, given that changes in neural activity induced via feed-back are assumed to differ mostly in the upper laminae [77], choosing the right acquisition and analysis approaches are crucial for studying cognitive processes within the cortical laminae.

Our findings indicate that post-processing steps, such as distortion-correction, can indeed affect the laminar signal of interest. Registration errors can, in principle, add to the differences between the two workflows. As we did not observe a systematic shift of the registration between the GM masks of the two approaches, registration errors cannot account for these observations. The lower dynamic range in the Conventional workflow, however, could be a consequence of the recalculation of the voxel intensities and smoothing during the distortion-correction step. Thus, both the reduced BOLD signal amplitude in the superficial layers and increased BOLD signal amplitude in the deeper layers for the Conventional approach are most likely due to the smoothing introduced by distortion-correction rather than registration errors. It is important to note that the responses were measured from 
a subset of voxels that was active for both stimuli. This ensured that there were no differences in the underlying vasculature amongst the voxels being averaged and compared across both conditions.

\section{Mid-GM, CSF and venous time-courses}

Given the high-resolution of our data, we were able to identify (Fig. 2.7) and extract the event-related haemodynamic responses from veins and CSF voxels and compared them to the response in mid-GM (Fig. 2.11). The CSF voxels with significant functional activity in the present study most likely refers to voxels that contain smaller pial veins which are embedded in the CSF but are not resolvable given the spatial resolution utilised in this study. Although the PB response appears to be faster in the mid-GM, which would be consistent with the fact that the neuronally-driven haemodynamic response in GM is expected to exhibit faster dynamics than the venous response [49, 168-172], we did not find a statistically significant difference in the times-to-peak between the time-courses. Interestingly, a stronger PSU is observed in the mid-GM [173] compared to the venous and CSF responses having little or no undershoot. Our finding argues that the PSU is better localised relative to the neuronal activity than the PB response [132, 174, 175]. Importantly, the differences in the PSU for the two stimuli are best visible in the GM rather than on the surface. Therefore, the fMRI signal from the pial veins can smooth out the tissue fMRI response. Given that surface fMRI signals are larger in amplitude, our finding indicates that low-resolution fMRI studies may be less sensitive to true differences in neuronal activity, which are better represented by the tissue fMRI signals, thus, further emphasising the need for ultra-high field high-resolution imaging. In the case of low-resolution GE fMRI studies, however, excluding the highest activated voxels representing signal from the pial veins may 
increase specificity for tissue specific signal that could more closely represent neuronal processing $[68,154,156,176]$.

\section{Relative ROI size analysis}

Most laminar fMRI studies rely on ROI-based analyses making the acquisition strategy and the subsequent data processing steps critical to investigate signal modulations across laminae. For large ROIs, small errors in registration and signal blurring that result in laminar differences between the workflows can effectively cancel out because of averaging over a large cortical region. However, in most laminar cognitive neuroscience studies, the investigated effect of interest occurs in small patches along the cortex. To this end, we investigated the laminar differences between workflows by varying the sampled area, i.e. the ROI size

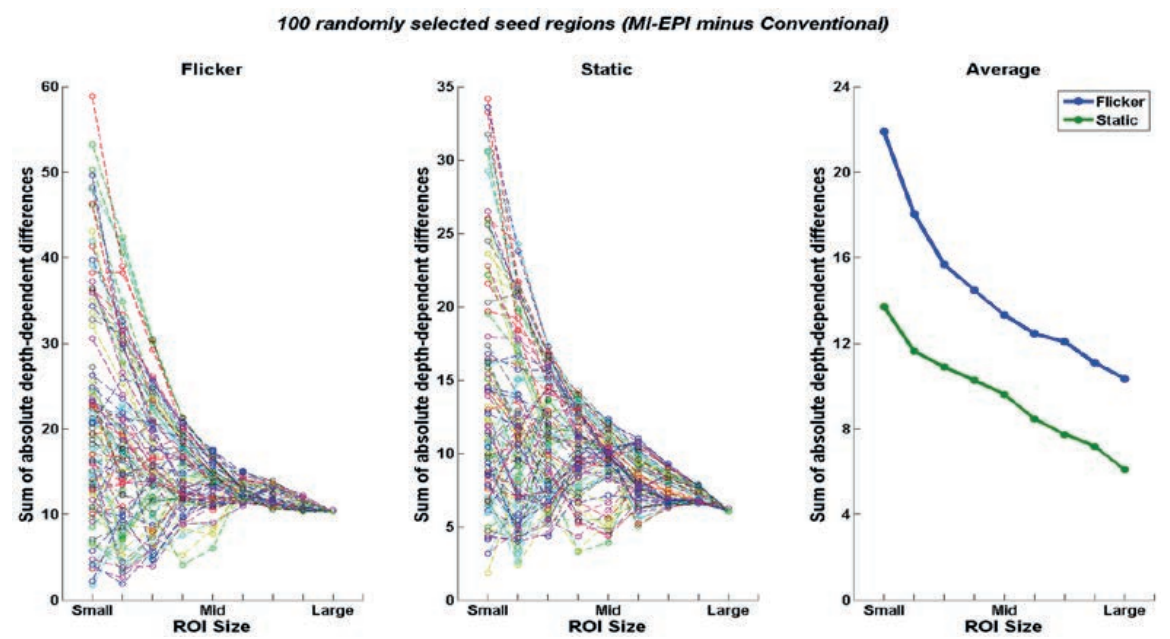

Figure 2.15: Left-to-right: Each coloured line represents the ROI size dependence profile of the sum of absolute differences of laminar PB (MI-EPI - Conventional) from a random seed region in an example GM ROI for flicker and static stimuli, and the average of all the $100 \mathrm{ROI}$ size-dependence profiles. 
(Fig. 2.12), for both stimulus conditions. Because most laminar fMRI studies are interested in differences between tasks, the choice of the seed ROI was based on the voxels having the largest difference between stimulus conditions and not the highest activated voxels. The quantitative metric for the comparison was the absolute sum of differences in signal between the two workflows. We observed that the signal difference between the workflows is largest for the smallest ROI as this region has the highest signal change given our seed ROI criteria. By increasing the ROI size, i.e. averaging more voxels around the seed ROI, the laminar difference between workflows steadily decreases. As an additional test, we bootstrapped the seed of our ROI, performed the same analysis and observed that the effect of increasing ROI size is very similar (Fig. 2.15) to those seen in Figure 2.12. This supports the argument that, in studies mapping large brain regions, the choice of workflow would not be critical. However, for laminar fMRI studies, wherein small patches of cortex are typically of interest, the choice of analysis workflow does have an impact on the laminar signal being sampled.

\section{Laminar profile normalisation}

The GE signal is largely biased by veins oriented parallel (pial) and, to a smaller extent, perpendicular (ascending) to the cortical surface [43, 154, 156, 177, 178]. The underlying laminar BOLD signal is biased mainly by (a) draining of deoxy-Hb from lower to upper layers of the cortex via ascending veins and (b) laminar differences in baseline CBV. Although the first bias is more pronounced with GE, the ascending veins can contribute to the degradation of the laminar signal specificity in SE as well. In this regard, initial steps towards model-based removal of this bias have been carried out [109, 110]. Here, we propose to reduce the latter bias by dividing laminar signals between conditions and/or time points and motivate 
this approach with theoretical considerations derived for one-vascular compartment models [159, 160], which is appropriate for GE fMRI [43]. By taking the ratio of $\mathrm{PB}$ profiles from the different stimulus conditions with the MI-EPI workflow, the modulation of laminar signal could be observed (Fig. 2.13a), in contrast to the Conventional workflow. In the second case, using the ratio of different portions of the time-course, we were able to reveal laminar modulation of the PSU relative to the PB (Fig. 2.13b). The laminar profile in the MI-EPI workflow for the flicker condition shows that the PSU is relatively stronger in the middle to deeper layers, consistent with results obtained on animal models [132, 162]. However, this effect is not observed with the Conventional workflow. For the flicker condition using the MI-EPI workflow, we observe that the PB (Fig. 2.10) is proportionally larger in the superficial layers whereas the PSU (Fig. 2.13b) is relatively (but not absolutely) larger with increasing depth. The PSU and PB profiles represent two distinct temporal stages in the processing of the stimulus information and our data indicate that their ratio has a different laminar specificity than the PB.

\subsection{Limitations and future considerations}

Our implementation of MI-EPI was time intensive $(\approx 25 \mathrm{~min})$ because of the multiple inversions and repetitions acquired. In future studies, the MI-EPI can be made more efficient and optimised [131] to significantly reduce the acquisition time. Alternative approaches to acquire sub-millimetre distortion-matched anatomy at 7 $\mathrm{T}$ include the $\mathrm{T}_{1}-2$-3DEPI [179] and $\mathrm{T}_{1}$-EPI [180]. Despite the fact that the MIEPI can help improve co-registration accuracy and provide anatomical contrast to delineate cortical layers, it is still an additional run to acquire. It would be ideal to use the endogenous anatomical contrast of the functional data itself. Re- 
cently, laminar fMRI studies using VAscular Space Occupancy (VASO) to measure functional CBV changes [96, 180] show that it is possible to calculate anatomical $\mathrm{T}_{1}$ contrast from the functional VASO data itself, thereby, circumventing the need to co-register datasets and distortion-correct altogether. However, this is currently not possible for standard BOLD fMRI studies and maybe future work could explore whether adding specialised RF pulses or analysing additional features of fMRI data, such as the phase or temporal standard deviation, may aid the segmentation of the GM ribbon in EPI space without the need for an additional anatomical run. Until then, our workflow using the MI-EPI provides a good alternative to conventional approaches to acquire and analyse high-resolution laminar fMRI at ultra-high fields. Additionally, it is possible that the GM thickness following distortion-correction in the functional data may be different than the MP2RAGE given the differences in phase-encode and readouts between the functional and anatomical datasets. This difference in GM thickness between the MP2RAGE and the functional (EPI) data can cause inaccurate cortical depth definitions even in the case of accurate co-registration and, therefore, inevitably cause incorrect sampling of the laminar signal. While we did not test for this, it does not invalidate our conclusion that the MI-EPI workflow would not only overcome the first limitation of having to distortion-correct the data but also, given the quantitative nature of the anatomical map, more accurately represent the GM in the native EPI space.

Segmentation of GM, WM and CSF remains a challenge because most available data analysis packages are limited to automated whole-brain segmentation and need to be adapted for data with partial coverage. Although the GM ribbon can be reliably delineated due to the good anatomical contrast in both workflows, the sampled functional signal is still dependent on the processing strategy applied and 
the accuracy of co-registration. Additional analyses using GM segmentation from the distorted MP2RAGE and functional data in native EPI space demonstrate that the differences between the two workflows are mostly due to blurring induced by the distortion-correction in the Conventional workflow, rather than misregistration (Fig. 2.16), resulting in slightly higher BOLD amplitude in the deeper cortical depths and lower in the upper layers in the Conventional approach compared to the MI-EPI approach. Each study should be carefully examined for confounds in the BOLD signal profiles resulting from both distortion-correction and registration related effects. Although we did not systematically investigate this effect, it is important to note that distortions in the phase-encoding direction can also introduce some blurring across layers and must be considered when selecting the acquisition orientation. It is important to carefully acquire the data such that the phase-encode blurring is preferentially within, rather than across the layers in an ROI that is activated by the stimulus [96].
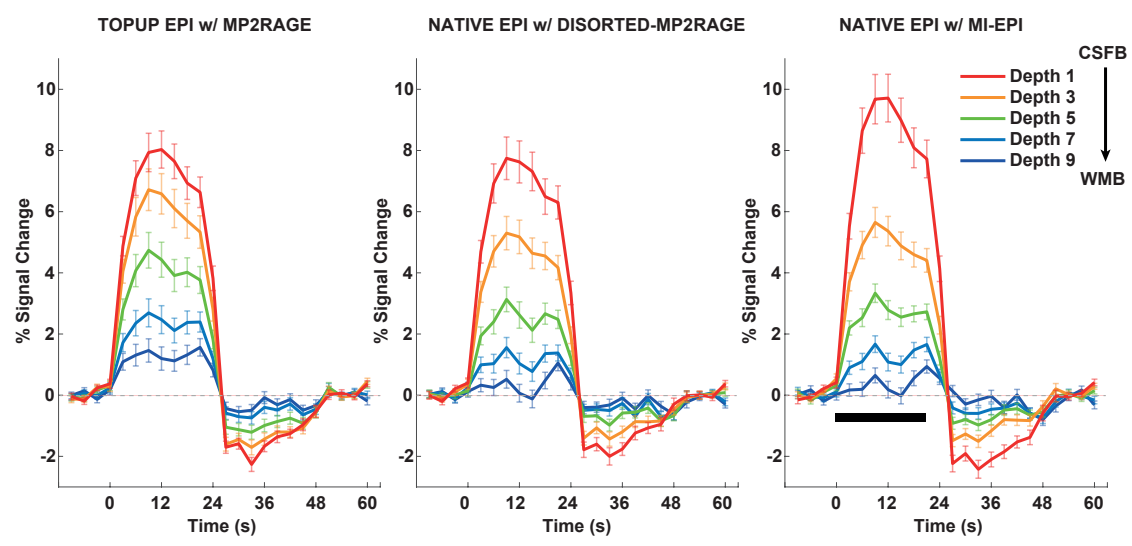

Figure 2.16: Event-related average laminar time-course obtained using the distorted-MP2RAGE (middle). Time-courses from the MI-EPI workflow (right) and the Conventional workflow (left) are presented on its either side for comparison. Error bars indicate SEM. 
Despite the recent developments in high-resolution acquisition using different functional neuroimaging modalities such as CBV [166] and CBF [88], GE BOLD fMRI remains the workhorse for high-resolution laminar neuroimaging. In general for GE BOLD fMRI studies, care must be taken in interpreting laminar signal changes in the upper layers in terms of neural processing [181]. In this study, we propose a normalisation approach based on existing BOLD signal models [159, 160] to uncover laminar non-linearities of the GE BOLD signal. Note, however, the normalisation approach still does not fully account for all the complexities of vascular biases in laminar fMRI and, thus, we emphasise the need for a more comprehensive BOLD signal model to remove those biases in sub-millimetre laminar fMRI studies. Thus, we propose the normalisation approach as a tool to remove baseline parameter biases and detect non-linearities, most likely of neuronal origin, rather than accounting for all vascular biases in the laminar fMRI signals. Interpretations of normalised profiles need to be carefully considered because division by small numbers (for example, reduced GE BOLD SNR towards the WM boundary) may over-estimate the relative signal change in the deeper layers compared to the superficial layers. Another important aspect for ROI-based analysis is that the BOLD signal is assumed to not vary tangentially within the analysed segment at each cortical depth. This assumption of invariance is why voxels are averaged throughout the ROI in a laminar manner. However, future studies must consider studying the signal variation along the cortical sheet for their significance in cortical processing and initial attempts, such as the cortical laminagram [72], show promise. Future studies can consider using a spatial GLM to sample the laminar fMRI signal $[72,161,182,183]$ wherein the signal within each voxel is modelled as a linear combination of signal contributions from different layers. The spatial GLM approach has been shown to have a higher specificity compared to the 
commonly used resampling approach [72]. However, more work needs to be done in order to improve and validate the spatial GLM approach for high-resolution laminar fMRI.

\subsection{Concluding remarks}

In this study, we presented and evaluated a processing workflow for laminar analysis of sub-millimetre spatial resolution GE BOLD fMRI data at $7 \mathrm{~T}$ using a distortion-matched $\mathrm{T}_{1}$ map from MI-EPI. We show that minimally processed fMRI data can be analysed in its native EPI space and this is facilitated by acquiring distortion-matched anatomical contrast using MI-EPI. We demonstrate that the distortion-correction methods can reduce the specificity of the laminar fMRI signal. We confirm that the PSU is spatially better localised in GM relative to the positive BOLD, and is proportionally larger in the GM than the veins. In addition, we provide a theoretical basis and demonstrate that the laminar modulation of the neuronal signal can be, at least partially, recovered from GE BOLD signal by dividing the depth profiles from the different conditions or portions of the time-course. In conclusion, the choices made for acquisition strategy, processing and analysis in high-resolution laminar fMRI data can significantly impact the observed layer-specific effects and the possible outcome of studies. A comprehensive modelling, acquisition and analysis approach is crucial to deconvolve the neuronal activation from the laminar BOLD fMRI signals that are coupled to each other across layers. 


\section{Acknowledgements}

The study was supported by the Netherlands Organisation for Scientific Research (NWO) VIDI grant to Kâmil Uludağ (452-11-002). We thank Laurentius Huber for the implementation of the tr-FOCI inversion pulse in the MI-EPI sequence, Jose P. Marques for providing the $\mathrm{T}_{1}$ correction scripts, Roy A. M. Haast and Pierre-Louis Bazin for the anatomical data processing tips, Robert Turner for the literature recommendation and encouraging feedback at ISMRM 2016, and the anonymous reviewers for the critical reading and review of the manuscript. 



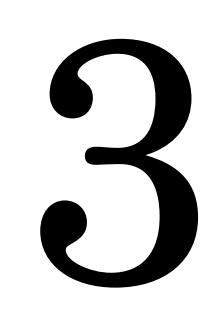

\section{Ultra-high resolution fMRI using anisotropic}

voxels

Associated publication: S. Kashyap, D. Ivanov, M. Havlicek, S. Sengupta, B. A. Poser, and K. Uludağ. "Resolving laminar activation in human V1 using ultra-high spatial resolution fMRI at 7 T". in: Scientific Reports 8.1 (2018), p. 17063. 



\begin{abstract}
The mesoscopic organization of the human neocortex is of great interest for cognitive neuroscience. However, fMRI in humans typically maps the functional units of cognitive processing on a macroscopic level. With the advent of ultra-high field MRI ( $\geq 7 \mathrm{~T}$ ), it has become possible to acquire fMRI data with sub-millimetre resolution, enabling probing the laminar and columnar circuitry in humans. Currently, laminar BOLD responses are not directly observed but inferred via data analysis, due to coarse spatial resolution of fMRI (e.g. 0.7 - 0.8 $\mathrm{mm}$ isotropic) relative to the extent of histological laminae. In this study, we introduce a novel approach for mapping the cortical BOLD response at the spatial scale of cortical layers and columns at $7 \mathrm{~T}$ (an unprecedented $0.1 \mathrm{~mm}$, either in the laminar or columnar direction). We demonstrate experimentally and using simulations, the superiority of the novel approach compared to standard approaches for human laminar fMRI in terms of effective spatial resolution in either laminar or columnar direction. In addition, we provide evidence that the laminar BOLD signal profile is not homogeneous even over short patches of cortex. In summary, the proposed novel approach affords the ability to directly study the mesoscopic organization of the human cortex, thus, bridging the gap between human cognitive neuroscience and invasive animal studies.
\end{abstract}





\subsection{Introduction}

The human cortex is structured into a myriad of structural and functional units [185]. These units can be indexed along both the normal and tangential coordinates with respect to the surface of the cortex [186]. The normal coordinates represent the cortical depth, from the pial to the white-matter boundary (WMB), also called cortical layers or laminae. The tangential coordinates represent the distance along the cortical ribbon, and the structural-functional units along this axis are called brain areas and columns, on the macroscopic and mesoscopic scales, respectively. These subdivisions are characterized by their distinctive histological profiles $[58,90]$ or by their specific responsiveness to external stimuli and role in cognitive and physiological processes [187, 188]. Specifically, functional units are typically assessed in animal models using invasive methods, such as electrophysiological recordings [189] and intrinsic signal optical imaging [190]. Based on invasive animal studies, a microcircuit model of the cortex has been proposed with differential roles of cortical layers in feed-forward processing of stimuli and cognitive feed-back [60]. In contrast, human cognitive neuroscience usually assesses only macroscopic functional organization due to limitations in spatial resolution. For example, data using functional magnetic resonance imaging (fMRI), the most popular non-invasive human neuroimaging technology, are typically acquired at 1.5 $\mathrm{T}$ and $3 \mathrm{~T}$ with voxel dimensions of around $3 \mathrm{~mm}$, which is about the average cortical thickness.

FMRI has proven to be an invaluable non-invasive imaging tool for mapping brain areas involved in cognitive and/or sensory processing [191] or resting-state activity [192]. It typically utilizes the blood oxygenation level-dependent (BOLD) contrast $[35,38]$ to probe neuronal activity indirectly via changes in blood oxygenation and cerebral blood volume (CBV) [49]. The increased availability of 
ultra-high field (UHF) human MRI scanners $(\geq 7 \mathrm{~T})$ and the development of novel fMRI acquisition techniques (see Poser and Setsompop [55] and references therein) have led to routinely achieving sub-millimetre spatial resolutions and thus, allow the non-invasive examination of laminar structures responsible for functional processing in humans (for reviews, see Lawrence et al. [193], Dumoulin et al. [194]). Most UHF fMRI studies in humans probing laminar circuitry thus far have utilised the BOLD signal as the functional contrast $[66,67,73,77$, $89,93,95,98,153,195]$. Recently, in addition to the BOLD contrast, CBV measurements using the VASO technique have also been utilised to study functional laminar profiles in the human cortex [82]. Other contrast mechanisms, such as using cerebral blood flow (CBF) and oxygen metabolism $\left(\mathrm{CMRO}_{2}\right)$, for laminar fMRI in humans are also currently under development (for a recent review see Huber, Uludağ, and Moller [80]). Currently, all these alternatives to GE-BOLD have lower signal-to-noise (SNR), contrast-to-noise (CNR), spatial resolution and brain coverage.

However, data acquisition and analysis challenges for high-resolution fMRI remain, which hamper the direct interpretation of the measured fMRI signal in terms of layer- and columnar-specific neuronal activity. Firstly, although fMRI is the non-invasive technique with the highest spatial resolution, the voxel size (e.g. $0.7-0.8 \mathrm{~mm}$ isotropic) still is large compared to the spatial dimensions of cortical layers and columns. Consequently, to infer the underlying layer-specific fMRI signal, the functional data are typically spatially upsampled using various interpolation techniques and averaged over an extended region-of-interest (ROI) [75]. This approach demands very high accuracy in segmentation of the cortical sheet and co-registration of anatomical-functional data and relies on the assumption that the depth-dependent fMRI signal along the cortical distance (i.e. tangential 
direction) is identical within the ROI. An alternative approach is sorting of voxels according to the relative distance of their centroids from the cortical boundaries $[67,75]$ (also see section 3.2.5). Secondly, the BOLD signal stems from changes in blood oxygenation (and CBV) mainly in the post-capillary vascular compartments. Cortical ascending veins drain the blood from individual cortical layers towards the cortical surface, which creates contamination of the local layer-specific fMRI signal with non-local signal changes stemming from the lower layers (i.e. closer to the WMB). This leads to a BOLD signal spatial profile [89, 94, 195] remarkably incongruent to electrophysiological laminar profiles [49]. Please note that the current work focuses on the first acquisition-related issue, with the aim of achieving ultra-high spatial resolution capable of directly resolving the laminar fMRI signal and thus, removing the need for upsampling and averaging over an extended ROI. To address the second physiology-related issue, several modelling attempts [51, 109, 110] have been proposed to remove the contribution of the nonlocal effects due to ascending veins to the measured (steady-state or dynamic) fMRI signal.

Inspired by line-scanning fMRI in animals [79], this study showcases a novel layer-specific fMRI acquisition strategy in humans at $7 \mathrm{~T}$ that can sample the cortical depths at an unprecedented spatial resolution (i.e. $0.1 \mathrm{~mm}$ ) using unique highly anisotropic voxels (Fig. 3.1, right). We demonstrate the feasibility of the novel Anisotropic Voxel FLASH (AVF) acquisition to obtain laminar- and columnarspecific fMRI signals in the human visual cortex. Furthermore, we show that the layer-specific BOLD signal mapped by the AVF within a single 'voxel-column' is highly stable and can thus, reliably capture layer-specific changes in small patches along the tangential cortical distance. As layer-specific profiles can be obtained without averaging over an extended ROI, we are able to detect, for the first time, 


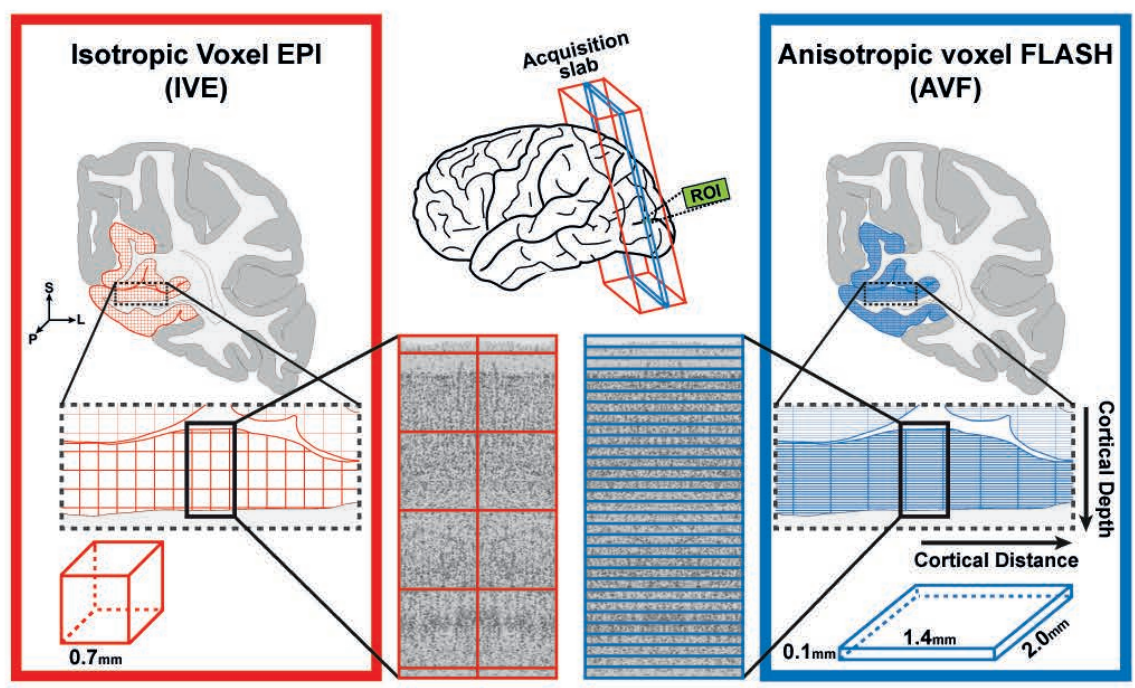

Figure 3.1: Illustration of the IVE (left, red) and the novel AVF (right, blue) acquisition schemes for layerspecific fMRI, highlighting the differences in the sampling of cortical depths between the two approaches.

a remarkably high variability of laminar profiles along the cortical distance, which is difficult to detect with the standard Isotropic Voxel EPI (IVE) and even with invasive electrophysiology. Finally, it is generally believed that the IVE acquisitions can, in principle, achieve any effective super-resolution desired via post-processing steps if there are enough voxels within the ROI and each of the sub-millimetre voxels sample the cortical layers depths in a quasi-random fashion, i.e., there is a spatial jittering of the voxels relative to the cortical layers. We argue and demonstrate using simulations that the effective laminar spatial resolution of the IVE acquisition is much lower than the AVF, even if an extended ROI is utilized to derive cortical depth profiles.

Nevertheless, we do not propose the AVF approach as a standalone, but rather as a supplemental tool that can be used to aid the interpretation of the depth- 
dependent or lower-resolution signal changes observed with isotropic sub- or supramillimetre acquisitions, typically used in cognitive neuroscience applications. Taken together, this study demonstrates the feasibility of directly assessing the laminar and columnar cortical organization in humans, non-invasively, using ultra-high spatial resolution fMRI, a scientific domain previously reserved for invasive animal studies.

\subsection{Methods}

\subsubsection{Participants}

Seven healthy volunteers (median age=28 years, 3 female) participated in the study following screening and having given written informed consent. The study was approved by the Ethics Review Committee for Psychology and Neuroscience (ERCPN) at Maastricht University and all procedures followed the principles expressed in the Declaration of Helsinki.

\subsubsection{Stimulus paradigm}

Full contrast black-and-white radial flickering ( $8 \mathrm{~Hz}$ ) checkerboard was presented using PsychoPy $[136,196]$ for $20 \mathrm{~s}$ (stimulus on) followed by $40 \mathrm{~s}$ of an isoluminant grey background (stimulus off). Each functional run lasted 12 minutes consisting of a $30 \mathrm{~s}$ initial baseline period and ten stimulus on-off blocks. The participants were instructed to remain motionless and fixate on a central fixation dot throughout each functional run. 


\subsubsection{Region-of-interest (ROI)}

Similar to the line-scanning approach [79], the cortical patch selected as ROI was determined from a continuous 'flat' portion of the subject's calcarine sulcus (therefore, V1), with additional criterion that the ROI was approximately uniformly thick and stayed flat for about $2.0 \mathrm{~mm}$ along the slice direction. The ROIs were first identified in each subject based on an anatomical MP2RAGE reference scan from a previous MRI session to save time during the acquisition session of the fMRI data. Based on this prior knowledge of the ROI location, the session-specific MP2RAGE localizer was used to fine-tune the placement of the fMRI imaging volume.

\subsubsection{Data acquisition}

All data were acquired on a whole-body Magnetom 7T research scanner (Siemens Healthineers, Erlangen, Germany) using a custom-built $16 \mathrm{Rx} / 4 \mathrm{Tx}$ channel phasedarray visual cortex coil (Fig. 3.2) [197]. A single session consisted of a total of three anatomical scans (MP2RAGE localiser $_{\text {MP2RAGE }}$ reference, $_{\text {MI-EPI }}$ reference $)$ and a total of five functional scans. The sequence parameters, listed in Table 3.1, were optimised in pilot experiments.

In each session, a high-resolution MP2RAGE [117] (0.7 $\mathrm{mm}$ isotropic) was acquired first and used as an anatomical localizer for slice planning. The strong tissue contrast of the MP2RAGE was critical for positioning the imaging volume on the ROI for the functional scans. Three functional runs were acquired using an inhouse adapted FLASH [198] with anisotropic spatial resolutions $(0.1 \times 1.4 \times 2.0$ $\mathrm{mm}^{3}$ ) (called Anisotropic Voxel FLASH, abbreviated as AVF) along the readout and phase-encoding (matrix size 1200x86) directions. Factor of two GRAPPA un- 
Table 3.1: Table of optimised sequence parameters used in the study.

\begin{tabular}{|c|c|c|c|}
\hline Sequence & Resolution & Matrix size & Other parameters \\
\hline $\begin{array}{l}\text { 3D-MP2RAGE } \\
\text { (localiser) [117] }\end{array}$ & $0.7 \times 0.7 \times 0.7 \mathrm{~mm}^{3}$ & $320 \times 320$ & $\begin{array}{l}\text { FoV }=224 \times 224 \mathrm{~mm}^{2} ; 240 \text { sagittal slices; GRAPPA }=3 ; \\
\text { partial Fourier }=6 / 8 ; \text { Ref. lines } \mathrm{PE}=24 ; \mathrm{TR}=5000 \mathrm{~ms} \\
\mathrm{TE}=2.47 \mathrm{~ms} ; \mathrm{TI}_{1} / \mathrm{TI}_{2}=900 / 2750 \mathrm{~ms} ; \alpha_{1} / \alpha_{2}=5^{\circ} / 3^{\circ} ; \\
\text { Bandwidth }(\mathrm{BW})=250 \mathrm{~Hz} / \text { pixel; echo-spacing }=6.9 \mathrm{~ms}\end{array}$ \\
\hline $\begin{array}{l}\text { 2D-FLASH } \\
\text { (fMRI - AVF) [198] }\end{array}$ & $0.1 \times 1.4 \times 2.0 \mathrm{~mm}^{3}$ & $1200 \times 86$ & $\begin{array}{l}\text { FoV }=120 \times 120 \mathrm{~mm}^{2} ; \text { GRAPPA=2; Ref. PE lines }=64 ; \\
\mathrm{TR}_{\text {eff }}=1763 \mathrm{~ms} ; \mathrm{TE}=26 \mathrm{~ms} ; \alpha=12^{\circ} ; \mathrm{BW}=60 \mathrm{~Hz} / \text { pixel; } \\
\text { RF bandwidth-time product }(\mathrm{BWTP})=8.5 \\
\mathrm{RF} \text { Duration }=3000 \mu \mathrm{s}\end{array}$ \\
\hline $\begin{array}{l}\text { 3D-MP2RAGE } \\
\text { (anatomy) }\end{array}$ & $0.4 \times 0.4 \times 0.7 \mathrm{~mm}^{3}$ & $320 \times 320$ & $\begin{array}{l}\text { FoV=120x120 mm } ; 240 \text { sagittal slices; GRAPPA=3; } \\
\text { partial Fourier }=6 / 8 ; \text { Ref. lines } \mathrm{PE}=24 ; \mathrm{TR}=5000 \mathrm{~ms} \text {; } \\
\mathrm{TE}=2.42 \mathrm{~ms} ; \mathrm{TI}_{1} / \mathrm{TI}_{2}=900 / 2750 \mathrm{~ms} ; \alpha_{1} / \alpha_{2}=5^{\circ} / 3^{\circ} ; \\
\mathrm{BW}=280 \mathrm{~Hz} / \text { pixel} ; \text { echo-spacing }=7 \mathrm{~ms}\end{array}$ \\
\hline $\begin{array}{l}\text { 3D-EPI } \\
\text { (fMRI - IVE) [141] }\end{array}$ & $0.7 \times 0.7 \times 0.7 \mathrm{~mm}^{3}$ & $214 \times 214$ & 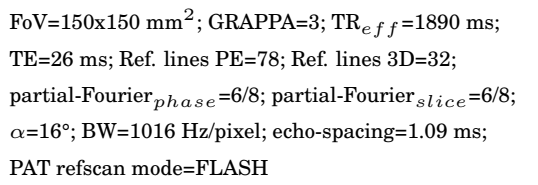 \\
\hline $\begin{array}{l}\text { MI-EPI } \\
\text { (anatomy) } \\
{[89,131]}\end{array}$ & $0.7 \times 0.7 \times 0.7 \mathrm{~mm}^{3}$ & $214 \times 214$ & $\begin{array}{l}\text { FoV=150x150 mm² } \text { GRAPPA=3; TR=9000 ms; } \\
\text { TE=29 ms; Ref. PE lines=63; partial-Fourier } \text { phase } \\
=6 / 8 ; \alpha=90^{\circ} ; \mathrm{BW}=1016 \mathrm{~Hz} / \text { pixel; echo-spacing } \\
=1.09 \mathrm{~ms} ; \mathrm{TI}_{1} / \mathrm{TI}_{2}=50 / 175 \mathrm{~ms} ; \text { TIs=64; Slab-size=2; } \\
\text { scan-time-per-slice=81.75 ms; Fat saturation; } \\
\text { PAT refscan mode=FLEET }[140] ; \alpha_{F L E E T}=10^{\circ}\end{array}$ \\
\hline
\end{tabular}

dersampling was used, resulting in a total of $43 \mathrm{k}$-space lines being acquired per slice at $\mathrm{TR}=41 \mathrm{~ms}$ (total slice $\mathrm{TR}=1.76 \mathrm{~s}$ ) and a flip angle of $12^{\circ}$. No partial Fourier was used to minimize blurring. At the echo time (TE) of $26 \mathrm{~ms}$, a readout bandwidth of $60 \mathrm{~Hz}$ per pixel could be afforded in order to maximize SNR. In addition, RF excitation with high bandwidth-time product (8.5) was applied to reduce slice profile imperfections. The high spatial resolution $(0.1 \mathrm{~mm})$ along the readout direction was selected so as to sample cortical depths, and therefore positioned orthogonal to the cortical surface in the ROI. The precise slice placement and orientation was thus adapted on a subject-by-subject basis, based on the curvature 

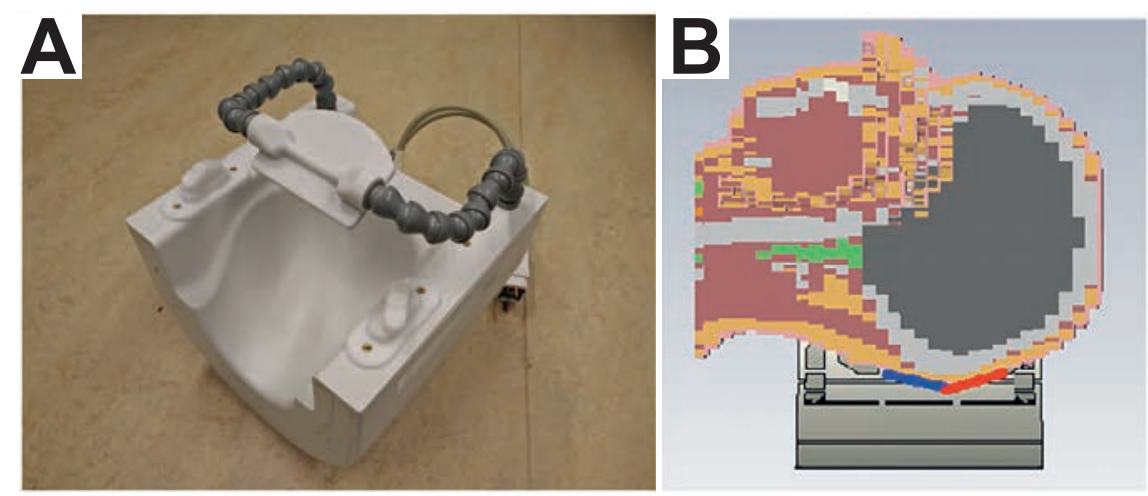

Figure 3.2: Specialized visual cortex RF coil array used in this study. (A) Assembled coil with mounted wide field-of-view mirror; (B) Two rows each of eight receive coil elements, (indicated in blue and red) encapsulate the occipital lobe (Adapted from [197] with permission).

of the calcarine sulcus, such that the phase-encoding direction is locally along the cortical distance (see Fig. 3.1). This is critical to prevent any phase-encoding dependent blurring from occurring across the cortical depths in the ROI. A second MP2RAGE with a high in-plane spatial resolution $\left(0.4 \times 0.4 \times 0.7 \mathrm{~mm}^{3}\right)$ was acquired with the same orientation as the AVF acquisitions. Subsequently, two functional runs were obtained using gradient-echo 3D-EPI [141] with isotropic spatial resolution (0.7 mm isotropic) (called Isotropic Voxel EPI, abbreviated as IVE), with the same FoV orientation as the AVF fMRI scans. Lastly, a multiple inversion recovery time EPI (MI-EPI) (0.7 mm isotropic) with 64 inversion times [89, 131] was acquired for anatomical reference, with the distortions matched to the isotropic 3D-EPI functional scans.

In order to further study the properties of the AVF acquisition, one of the seven subjects participated in an additional fMRI session using the same stimulus paradigm as in the main study. In this session, a high-resolution MP2RAGE was acquired as an anatomical localizer and was followed by an AVF run with highest-resolution 
sampling the cortical depth. The phase-encoding and frequency-encoding directions were then switched for the second and third functional runs, achieving the highest spatial resolution $(0.1 \mathrm{~mm})$ along the cortical distance, thereby sampling cortical "columns". Three additional functional runs were obtained using the AVF with laminar resolution but with increasing number of slices (i.e. increasing coverage), which resulted in consequently longer TRs [Run 1: Slices $=2, \mathrm{TR}_{e f f}=$ $3.53 \mathrm{~s}$; Run 2: Slices $=4, \mathrm{TR}_{e f f}=7.05 \mathrm{~s}$; Run 3: Slices $=6, \mathrm{TR}_{\text {eff }}=10.58$ s]. All other sequence parameters remained the same as in the main study (Table 3.1).

\subsubsection{Data processing and analysis}

\section{Functional data}

All AVF runs were motion-corrected with Advanced Normalization Tools (ANTs) $[199,200]$ using the time-series mean as the reference volume (Translation, antsRegistration). Each motion-corrected functional run was then carefully examined for realignment artefacts, and anatomical features, such as veins (low intensity voxels), were tracked on a volume-by-volume basis as a quality check. Statistical analysis of the fMRI data was done using FEAT 6.0 (FMRI Expert Analysis Tool), part of FSL (FMRIB's Software Library) [201]. The grand-mean normalized time-series was temporally filtered $\left(\sigma_{\text {high-pass }}=25 \times \mathrm{TR}, \sigma_{\text {low }- \text { pass }}=1.5 \mathrm{x}\right.$ TR) and statistical analysis was carried out using FILM with local autocorrelation correction [148]. The GLM model included a double gamma HRF with temporal derivatives and motion parameters as nuisance regressors. The within-subject higher-level analysis was carried out using a fixed effects model by forcing the random effects variance to zero in FLAME (FMRIB's Local Analysis of Mixed Effects) [149, 150]. The quan- 
titative $\mathrm{T}_{1}$ map from the second MP2RAGE (acquired with the same orientation as the AVF) was registered to the motion-corrected time-series mean AVF fMRI image using the registration tools in ITK-SNAP 3.6 [147]. Note that, in contrast to standard analysis in low-resolution fMRI studies, the anatomy was registered to the functional data in order to keep the functional data in its original space and, hence, avoid any additional smoothing [89]. The images were first aligned using the mutual information cost-function, and further manual fine-tuning of the alignment was performed using ITK-SNAP's interactive registration tool. The transformation matrix was exported in the ITK format and was applied to the anatomical data using ANTs (antsApplyTransforms, $4^{\text {th }}$ order B-spline interpolation). All IVE runs were also motion-corrected with ANTs using the time-series mean as the reference volume (Rigid, antsRegistration). No spatial smoothing or distortion-correction was applied (see MI-EPI workflow in [89]). Further statistical analysis of the fMRI data was done using FEAT 6.0, as described above. A distortion-matched $\mathrm{T}_{1}$ map was computed by fitting the anatomical MI-EPI data $[89,131]$.

\section{Segmentation of anatomical images}

ITK-SNAP 3.6 was used to semi-automatically segment the subject-specific MP2RAGE and MI-EPI $\mathrm{T}_{1}$ maps into three tissue classes based on $\mathrm{T}_{1}$ signal intensity: grey-matter (GM), white-matter (WM) and cerebrospinal fluid (CSF). These binary tissue class masks were manually corrected in ITK-SNAP after co-registration to the functional data, to ensure accurate delineation of the boundaries in the ROI and fix any co-registration artefacts. 


\section{Cortical depth analysis}

The AVF fMRI time-series data was imported into MATLAB R2015b (Mathworks, USA) for laminar analysis. The average GM thickness in the V1 ROIs across our subjects was $\approx 3 \mathrm{~mm}$, i.e. 30 voxels with $0.1 \mathrm{~mm}$ extent in the cortical depth direction. In order to be able to average cortical depth profiles over subjects with varying GM thickness, we linearly interpolated the total number of sampled cortical depths to 30 in each subject's ROI. For the IVE dataset, cortical depth analysis was done using CBS-Tools [202]. The CSF-GM and WM-GM boundary masks from the MI-EPI $\mathrm{T}_{1}$ segmentation were first converted to the levelset representation, and 30 equi-distant $[70,71]$ depths were defined in GM. The equidistant model of cortical layering was selected as it approximates to the equivolumnar model because our ROIs did not include regions of high cortical curvature [76].

To check whether similar results are obtained in both acquisition types, if the same ROI is considered, the cortical depth time-courses were averaged along the direction of the cortical distance for all subjects for both AVF and IVE acquisitions. The mean of the positive BOLD and post-stimulus undershoot signals were calculated by averaging over the time windows $12-26 \mathrm{~s}$ and $32-42 \mathrm{~s}$ following stimulus onset, respectively. The event-related cortical depth time-courses from both datasets were normalized to the mean positive BOLD signal change between $30-70 \%$ of cortical depth of each ROI for all subjects, in order to minimize bias to subjects with high BOLD signal changes. The normalized event-related time-courses were then averaged across subjects (Fig. 3.5a). The cortical depth analysis was always carried out using the data across the entire ROI (i.e. no statistical threshold or masking was used). 


\section{Variability along cortical distance}

Typically, in laminar fMRI studies [66, 67, 82, 95, 195], the sampled depth-specific signals are averaged tangentially along the cortical distance in an ROI. The principal reason for this being, due to the relatively large voxels $(\approx 0.7-0.8 \mathrm{~mm})$ relative to the histological layer thicknesses, the underlying laminar signals have to be inferred by averaging upsampled fMRI data and accounting for each voxel's partial volumes across the different depths (see sub-section, Effective spatial resolution, below). Due to this limitation, most studies are unable to assess the tangential variability of the acquired signal. In the AVF fMRI data, such upsampling and averaging over an ROI is in principle not necessary, assuming sufficient tSNR. Thus, we assessed the reliability of the AVF fMRI acquisition to probe the tangential variability using Principal Component Analysis (PCA) on the positive BOLD profiles in our ROI with the hypothesis that, if the profiles are similar over trials, then a PCA decomposition over trials should result in most of the variance in the data being explained by the First principal component (PC). For comparison, the PCA was also carried out for the IVE data in its native resolution.

\section{Effective spatial resolution}

It is generally assumed that, using the IVE fMRI data, upsampling and averaging over an extensive ROI can in principle yield super-resolved cortical depth profiles with effective spatial resolution only being limited by the extent of the ROI. In order to test this assumption and compare the effective spatial resolution of the IVE with the AVF acquisition, we simulated an anatomically-inspired sample of visual cortex with variable cortical thicknesses $(1.7-3.7 \mathrm{~mm})$ at $0.125 \mathrm{~mm}$ isotropic res- 

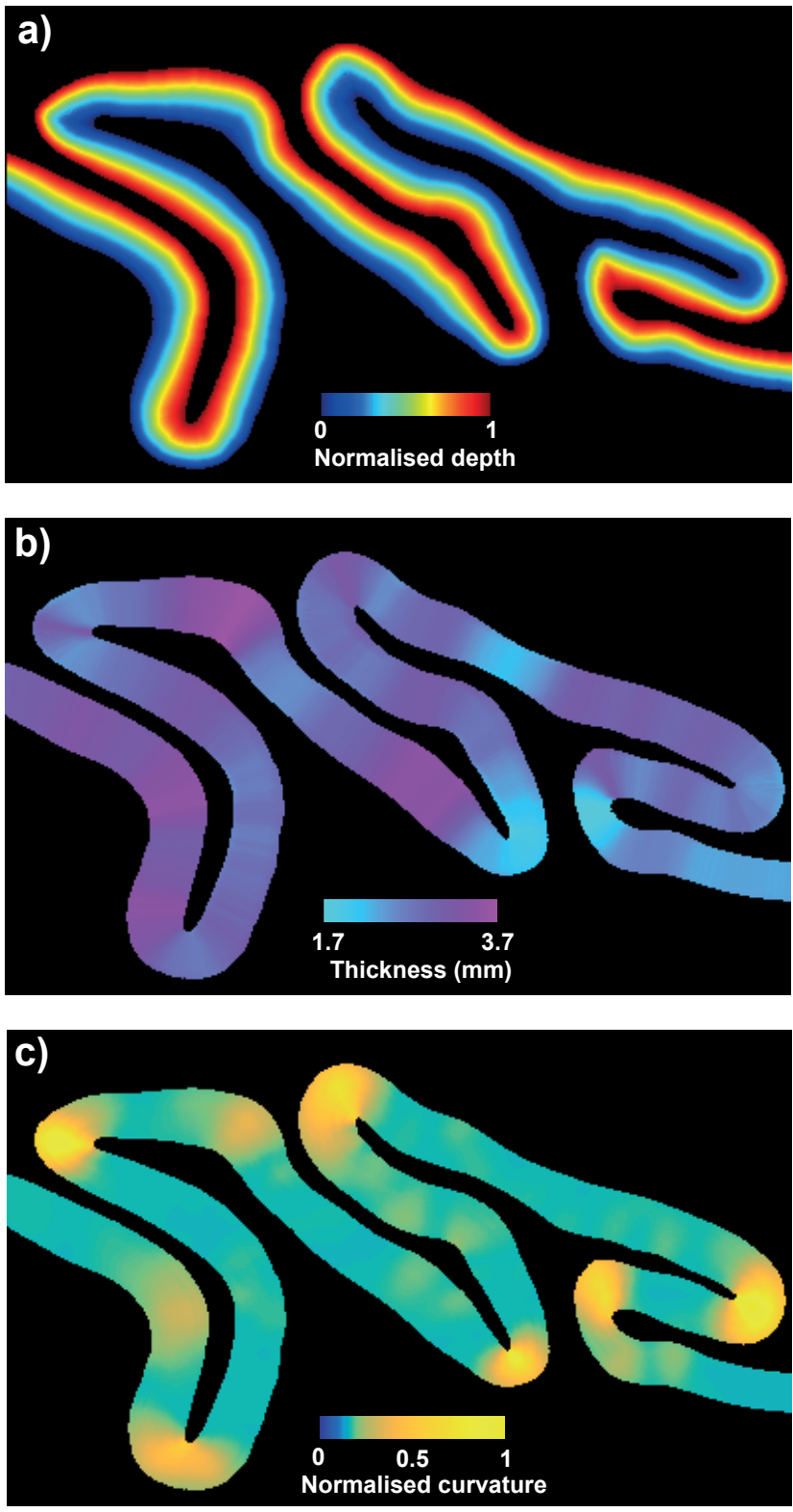

Figure 3.3: (a) Equi-volumnar layering, (b) variable cortical thickness and (c) normalised curvature in the anatomically-inspired patch of cortex [72] used for simulations. 
olution [72] (Fig. 3.3a). Twenty-one equi-volumnar cortical depths were defined, and voxel-partial-volumes were calculated for each cortical depth. Equi-volumnar model of layering was chosen here because of regions of high-curvature in the simulated cortex (Fig. 3.3b). Two fMRI datasets were generated assuming a Gaussian shaped activation profile in the middle of cortical ribbon with a full-width-athalf-maximum (FWHM) $1 / 5^{\text {th }}$ (Fig. 3.7) of the cortical thickness. The data was then downsampled (by averaging) to a $0.75 \mathrm{~mm}$ isotropic resolution. Next, we attempted to reconstruct the original activity profile by sampling the signal with three and twenty-one calculated cortical depths, respectively. Prior to layer sampling, the simulated functional data was upsampled using a nearest-neighbour interpolation method to $0.125 \mathrm{~mm}$ resolution and partial volume maps were calculated at this resolution for three and twenty-one numbers of cortical depths, respectively. Signal intensities of small voxels with corresponding partial volume greater than 0.75 within the cortical depth were averaged. We evaluated (a) the effect of the number of voxels (5 - 200) within an ROI with randomly assigned location along the cortical ribbon; and (b) the effect of additive noise, with $\operatorname{SNR}\left(\sigma_{S} / \sigma_{N}\right)$ $=1$ (i.e. realistic) and infinite (i.e. ideal) for two sampled number of cortical depth and widths of the activation profile. The point spread functions (PSF) were first reconstructed and the overlapping area under the true PSF was calculated to quantify blurring induced by subsampling the original signal. All simulations were repeated 200 times for both activation profile widths.

\subsection{Results}

The current study has two main goals: 1) To demonstrate the feasibility of cortical depth sampling using AVF. In order to show this, we compare the depth profiles obtained with AVF with those of IVE, typically utilized in laminar studies. 2) To 
examine the functional properties of AVF for studying cortical depth and distance profiles and using simulations, establish the superiority of the effective spatial resolution of AVF in two dimensions compared to IVE, albeit with lower brain coverage.

\section{Feasibility of anisotropic imaging for laminar fMRI}

In both the IVE and AVF acquisitions, activations in V1 were robustly detected in all subjects within single runs (illustrated in Fig. 3.4, left and right, respectively). In Figure 3.4, the zoomed-in panel illustrates an example ROI, showing the "flat" portion of the calcarine sulcus that was used for cortical depth analysis. The z-scores obtained from the GLM analysis are lower in the case of the AVF data, which is expected given the higher tSNR of the IVE data, partially due

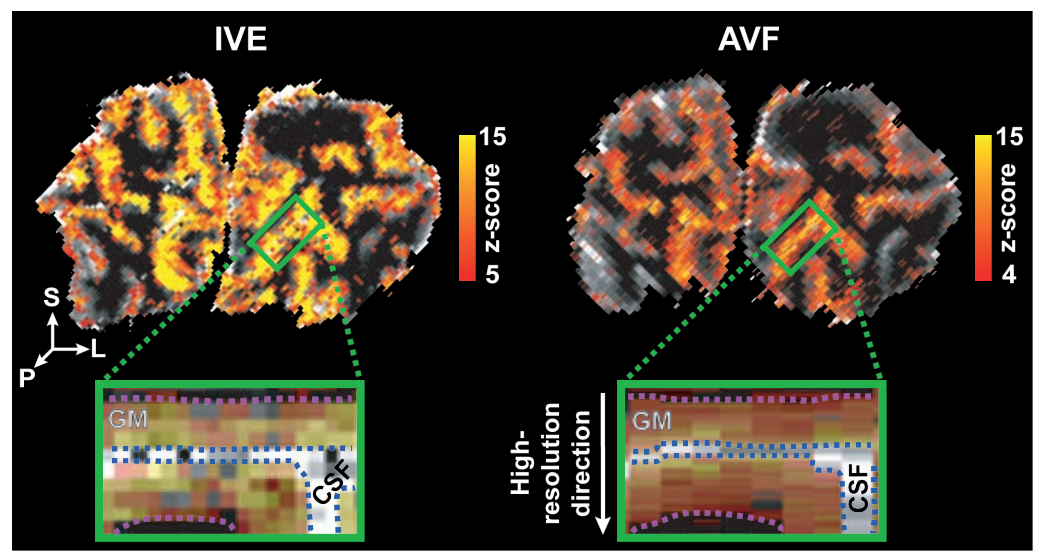

Figure 3.4: Example of single-subject, single-run activation maps for the flickering checkerboard stimulus, overlaid on the MI-EPI and the MP2RAGE $T_{1}$ maps for the IVE (left) and AVF (right) acquisitions, respectively. The green rectangle indicates the ROI in a coronal slice through the occipital lobe. In the zoomed-in panels, the dotted pink line indicates the GM-WM border and the blue line the GM-CSF border. In the activation maps, the values for CSF are masked out to better visualize the 'flat' cortical patch of interest. 
to the slightly larger voxel volume. The cortical depth time-courses for both acquisitions exhibit the typically observed steady BOLD signal amplitude increase towards the cerebrospinal fluid (CSF) boundary (Fig. 3.5) (see [49] for a recent review). In addition, strong post-stimulus undershoots are observed [89, 153] also increasing towards the CSF boundary. A statistically significant difference in the most superficial $36.7 \%$ of grey-matter (GM) can be observed between the positive BOLD cortical depth profiles (Fig. 3.5b, left) obtained with the AVF compared to the IVE $\left(\mathrm{F}_{(29,174)}=65.6, \mathrm{p}_{F D R}<0.0001\right)$. The post-stimulus undershoot profiles (Fig. 3.5b, right) also exhibit a statistically significant difference $\left(\mathrm{F}_{(29,174)}=8.231\right.$, $\left.\mathrm{p}_{F D R}<0.0001\right)$ between the two acquisition approaches, albeit only in the most superficial cortical depth.

\section{$\%$ BOLD variability along the cortical distance}

An interesting and consistent finding for the AVF data is the variability of the cortical depth profiles along the tangential cortical distance. Although the stimulus evokes a strong response in all voxels along the cortical distance, neighbouring voxels can be remarkably different, an observation, which is difficult to make using the IVE approach (or even using invasive electrophysiology). Thus, we investigated further whether this tangential variability stems from thermal noise (random) or is reproducible due to underlying (neuronal or vascular) physiological processes. The high degree of similarity between the patterns of positive BOLD signal profiles in an ROI from two example trials within a single AVF run is illustrated in Figure 3.6a. The tangential variability exhibits a high level of agreement between the two trials and, following the Principal Component Analysis (PCA) decomposition across trials, the first Principal Component (PC) retains the tangential variability. This suggests that the tangential variability in the AVF data 
a)
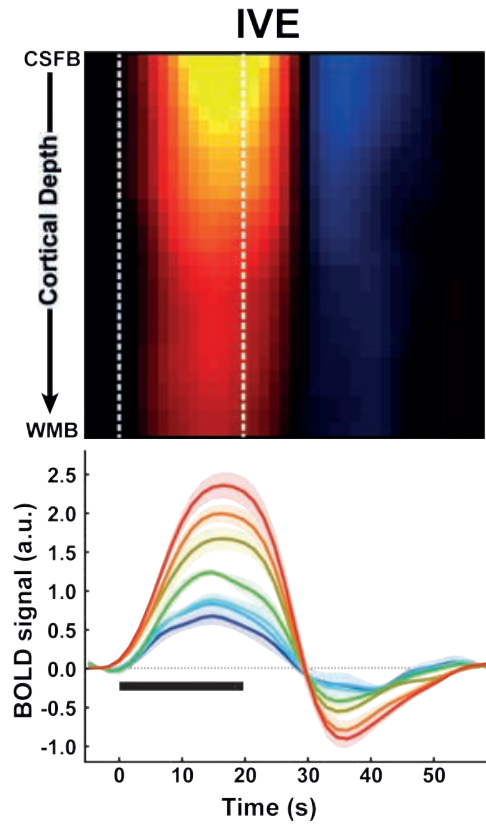

b)

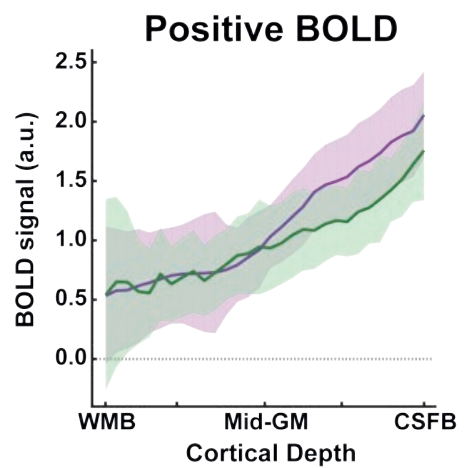

AVF

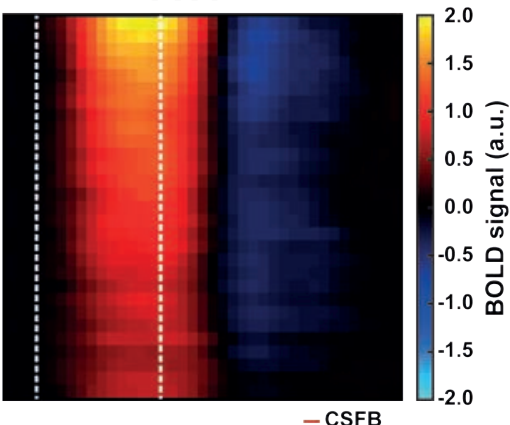

- CSFB

$-14 \%$ GM

$-31 \%$ GM

$-48 \%$ GM

$-66 \%$ GM

$-83 \%$ GM

-WMB

0 j

$0.5 \stackrel{1}{\frac{\pi}{3}}$

0.0 오ำ

0.50

.0 음

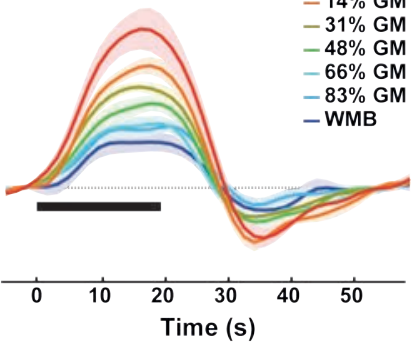

\section{Post-stimulus} undershoot

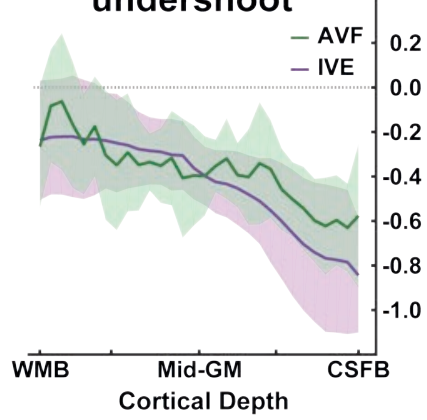

Figure 3.5: (a) Top: Colour-map representation of the average hemodynamic time-courses for 30 cortical depths from the IVE (left) and the AVF (right) acquisitions from CSF boundary (CSFB) to the white matter boundary (WMB). The white dotted lines on the colour-map indicate the stimulus-on period. Seven of the 30 cortical depth time-courses are presented below the heatmaps. (b) Cortical depth profiles of the average positive BOLD signal and post-stimulus undershoot for the two acquisition schemes. The BOLD signals have been normalised and the shaded regions represent the standard error of the mean (SEM). 
a)

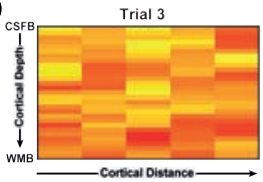

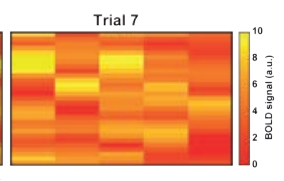

Principal Component 1

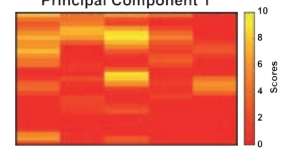

b)

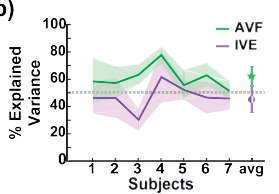

Figure 3.6: (a) (left to right) Average positive BOLD signal in the ROI of an example subject is shown for two trials in a single AVF run. The third panel shows the first PC obtained using PCA decomposition across all trials. (b) The percentage of variance explained by the first PC for all 7 subjects is plotted for the two acquisition approaches. The star and circle represent the group average percentage explained variance. The shaded regions and error bars indicate the standard error of the mean (SEM).

is reproducible, cortical variability. The percent variance explained by the first PC from the AVF data for the individual subjects (see Fig. 3.6b) is significantly higher compared to that of the IVE data (means: $61.3 \pm 8.31 \%$ and $48.9 \pm 9.32$ $\%$, respectively, $\mathrm{t}_{(11.79)}=2.708, \mathrm{p}=0.0193$ ), which was unexpected given the lower tSNR of the AVF data.

\section{Effective spatial resolution of the isotropic acquisition}

Figure 3.7a compares the induced PSF to the average reconstructed PSF for different number of sampled layers (simulated for the case of infinite SNR and maximum number of voxels). Figure 3.7b shows the dependence of the mean area under the induced PSF on the number of voxels. It reveals that, for the case of infinite SNR, relatively small number of voxels $(\approx 24$, e.g. 4 depth $\times 8$ distance voxels) are sufficient to reconstruct $61-68 \%$ of the induced PSF, with a higher percentage associated with the higher number of sampled layers. However, when simulated with added noise (SNR = 1, i.e., realistic for our fMRI data), more voxels are needed to reliably reconstruct the layer-specific activation profile. With less than 80 voxels (corresponding to $\approx 20$ voxels along the tangential cortical dis- 

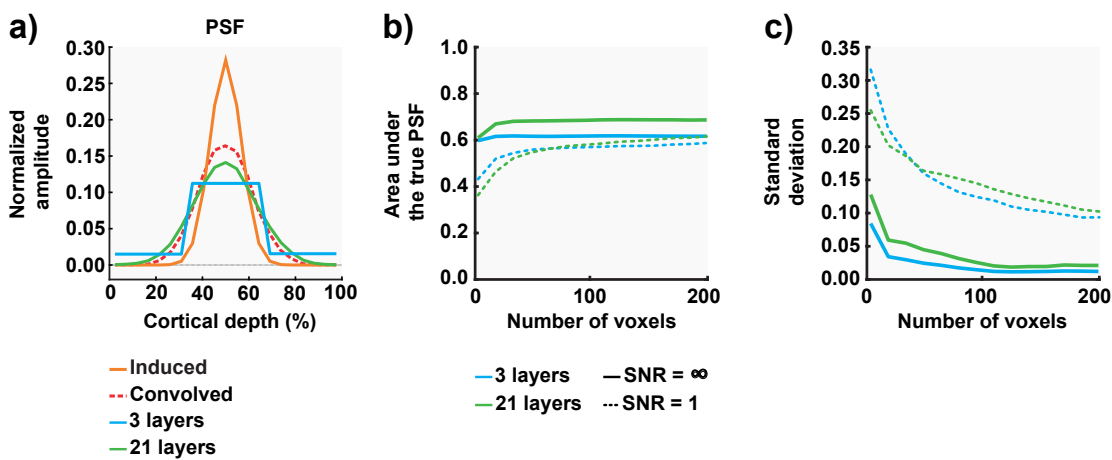

Figure 3.7: (a) The induced PSF used in the simulation (FWHM 1/5th of the cortical depth) is plotted together with the reconstructed PSFs obtained by convolving with kernel derived for the $0.75 \mathrm{~mm}$ voxels described in Koopmans et al. [67], sampling 3, 21 cortical depths with infinite SNR and large number of voxels (1000). (b) The overlap between the induced and reconstructed PSFs with the two different sampling strategies is quantified as a function of ROI size (i.e. number of voxels, profiles of 200 out of 1000 voxels are displayed) at two different SNR levels (ideal = infinite, realistic = 1). (c) The standard deviation of the reconstructed PSF from the induced PSF with increasing number of averaged voxels.

tance), lower number of layers are preferable, albeit with a very high standard deviation (Fig. 3.7c), hence resulting in low reliability of the estimated PSF. With ROIs larger than 100 voxels, sampling with higher number of layers can benefit the estimation of the underlying PSF. Importantly, even for the ideal case (SNR = $\infty$ ), increasing the size of the ROI ( $>100$ voxels) only minimally improves the estimated PSF, suggesting that the IVE acquisition, combined with standard analysis using upsampling of the functional data, is unable to fully recover the underlying physiological profiles.

\section{Simulating the large BOLD signal near the CSF/pial boundary}

To simulate the effect of leakage of the signal change in the pial vein to the cortical layers due to remaining partial volume effects, we considered activation (ampli- 
Without high curvature regions

a)

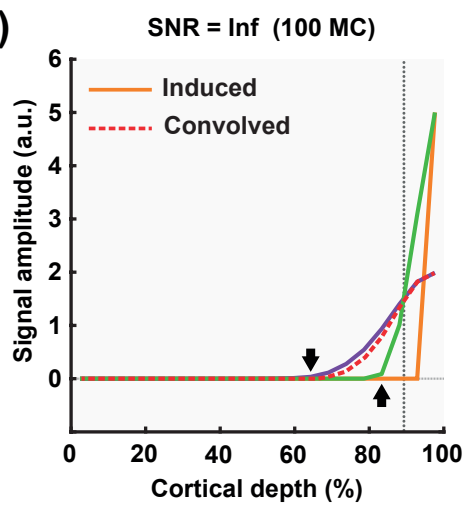

Only high curvature regions:

c)

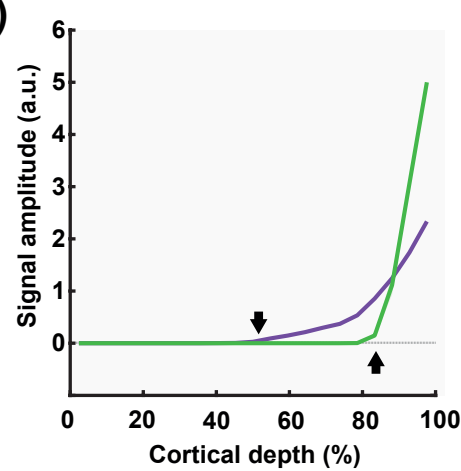

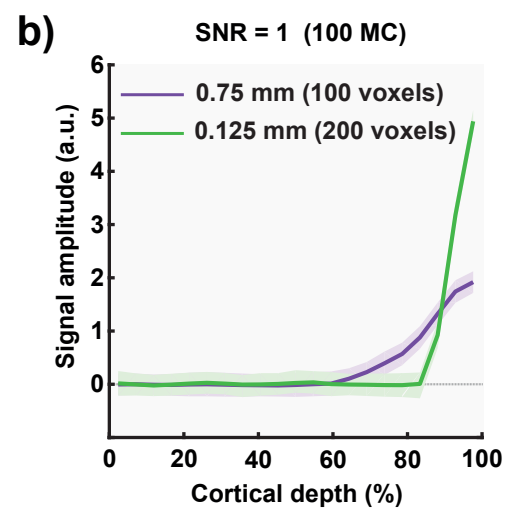

d)

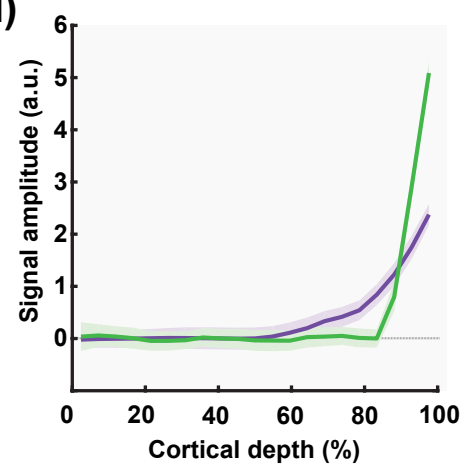

Figure 3.8: (a) The induced activation (amplitude = 5) in the uppermost cortical depth, plotted together with the reconstructed laminar profiles sampled from patches of cortex excluding high cortical curvature, obtained with $0.75 \mathrm{~mm}$ (purple) and $0.125 \mathrm{~mm}$ (green) isotropic voxels, respectively for the case of infinite SNR following 100 Monte-Carlo simulations. The red dotted line represents the induced laminar profile convolved with a convolution kernel derived for the $0.75 \mathrm{~mm}$ voxels as described in Koopmans et al. [67]. (b) Reconstructed laminar profiles for the different voxel sizes for the case of realistic SNR following 100 Monte Carlo simulations. (c-d) Reconstructed laminar profiles sampled from regions of high cortical curvature for the different voxel sizes for the case of infinite and realistic SNR, respectively following 100 Monte-Carlo simulations. 
tude $=5$ ) in the CSF/pial boundary and the very first cortical depth ( $1^{\text {st }}$ of 21$)$. The remaining lower 20 cortical depths were considered not to be activated. Two scenarios, with larger ( $0.75 \mathrm{~mm}$ isotropic) and smaller $(0.125 \mathrm{~mm}$ isotropic) voxel sizes, were compared. This represents a simplified analogy for the acquisition strategies (IVE and AVF) and their differences in laminar spatial resolution. The induced laminar profile (Fig. 3.8) was reconstructed by sampling the signal from twenty-one cortical depths and excluding regions with high-curvature (normalised curvature $>0.3$ ), having performed 100 repetitions of Monte Carlo simulation for noise-less $\left(\mathrm{SNR}_{\text {ideal }}=\right.$ infinite, Fig. $\left.3.8 \mathrm{a}\right)$ and additive noise $\left(\mathrm{SNR}_{\text {realistic }}=1\right.$, Fig. 3.8b) cases. Additionally, simulations were repeated using only regions of high-curvature (normalised curvature $>0.3)$. For larger voxels $(0.75 \mathrm{~mm})$, the estimated signal amplitude is lower at the surface and drops slowly, i.e. extending to $40 \%$ of cortical depth. For smaller voxels $(0.125 \mathrm{~mm})$, the estimated signal amplitude recovers the true amplitude level at the surface boundary and drops quickly within less than $20 \%$ of cortical depth. The reconstructed profiles (between $10-100 \%$ of cortical depth) in Figure $3.8 \mathrm{~b}$ are very comparable to the experimental results in 3.5 for the two acquisition approaches. If a convolution kernel, derived for $0.75 \mathrm{~mm}$ isotropic voxels as per Koopmans et al. [67], is applied to the induced laminar activation profile, the resulting reconstructed profile is very similar to the reconstructed profile obtained from the Monte Carlo simulations with $0.75 \mathrm{~mm}$ voxels (see Fig. 3.8a, red dashed line). In the case for highcurvature regions, the laminar profile for $0.75 \mathrm{~mm}$ voxels extends to deeper cortical depths ( 50\%), while the laminar profile reconstructed using $0.125 \mathrm{~mm}$ voxels is nearly identical to that obtained from the regions excluding high-curvature regions (Fig. 3.8b). 


\section{Mapping the BOLD signal along cortical distance with AVF acquisition}

Figure 3.9a shows a high degree of similarity in the single-subject, single-run statistical activation maps induced with the flickering checkerboard stimulus for acquisition with the highest resolution in the cortical depth (left) and cortical distance (right) directions, respectively. The z-scores from the regions demarcated in magenta ("laminar" acquisition) and cyan ("columnar" acquisition) dotted lines in the zoomed-in panels (Fig. 3.9a) are plotted in Fig. 3.9b. The magenta circles represent average z-score of 14 depths (a patch of $1.4 \mathrm{~mm}$ ) in the high cortical depth resolution acquisition from the same rectangular patch of cortex as sampled with the high cortical distance resolution acquisition. The cyan diamonds represent the z-score with a higher spatial resolution along cortical distance $(0.1 \mathrm{~mm})$. The data obtained with "laminar" acquisition closely tracks those of the average "columnar" acquisition. This adds further credence to the finding observed in Figure 3.6 that the tangential variability observed is not simply thermal noise but is most likely of physical or physiological origin. Interestingly, the BOLD activation z-scores along the cortical distance (cyan diamonds) change by two or three times, even over 0.1 $-0.2 \mathrm{~mm}$. 


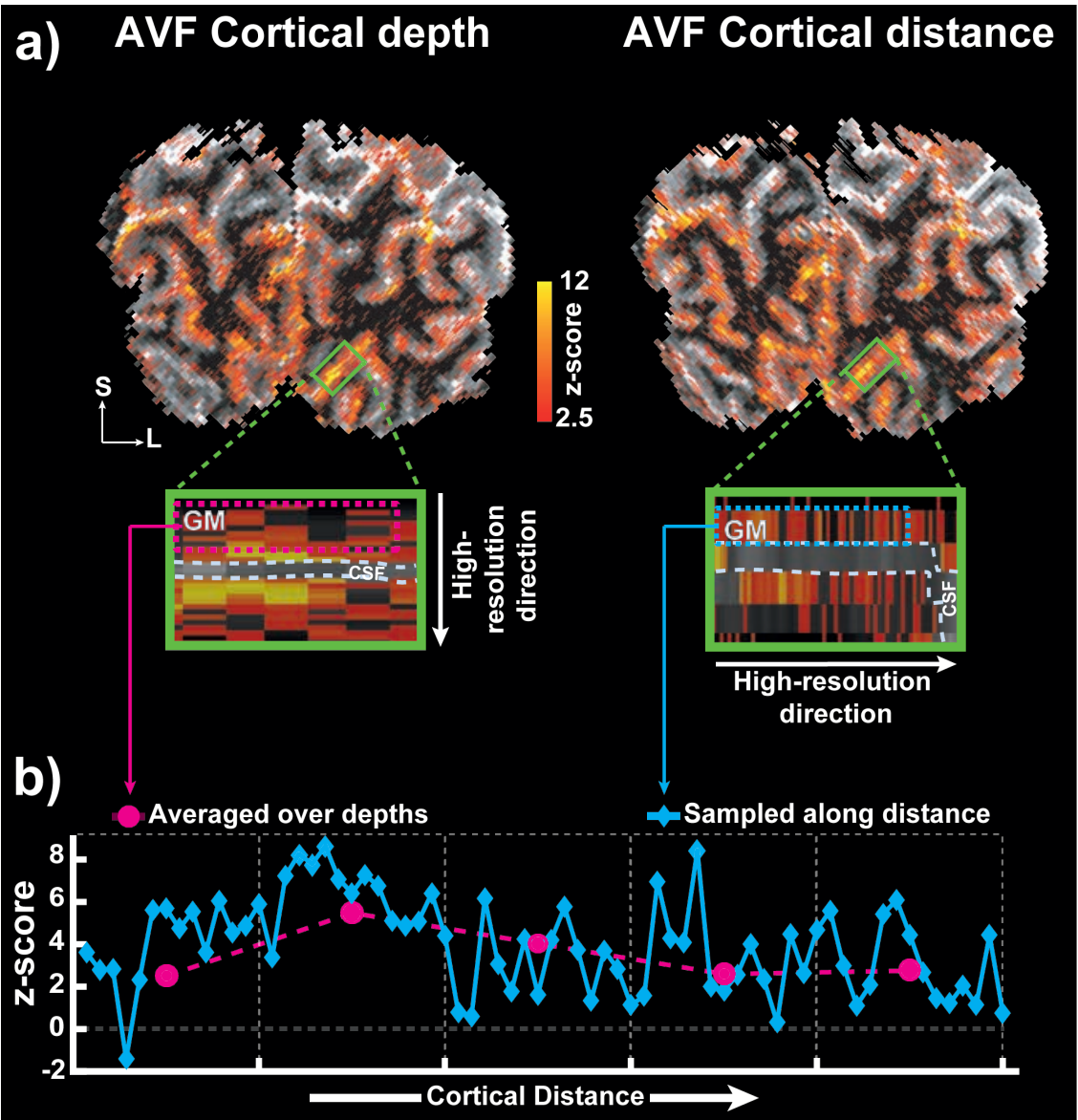

Figure 3.9: (a) Single-subject, single-run statistical activation map overlaid on the MP2RAGE T $_{1}$ map with the highest spatial resolution $(0.1 \mathrm{~mm})$ oriented along the cortical depth (left) and cortical distance (right). The green rectangle represents the ROI, and the zoomed-in panels highlight the high-resolution sampling of cortical depth and distance, respectively. (b) BOLD activation z-scores are plotted from the regions in magenta and cyan dotted lines in the zoomed-in panels from (a). 


\subsection{Discussion}

High-resolution fMRI has provided researchers the ability to map cortical depthdependent BOLD signal changes allowing addressing neuroscientific questions at the mesoscopic scale in humans. Cortical depth-dependent fMRI studies typically acquire voxel sizes of $0.7-0.8 \mathrm{~mm}$ isotropic (Fig. 3.1, left), with the isotropicity considered to be indispensable due to the gyrification of the human cortex. However, as the cortex is only 2 - $4 \mathrm{~mm}$ thick, even such high spatial resolution results in large partial volume effects over laminae and also with white matter (WM) and cerebrospinal fluid (CSF).

In this study, we demonstrated the feasibility of non-invasively acquiring laminar fMRI data in humans at an unprecedented spatial resolution of $0.1 \mathrm{~mm}$. To that end, we utilized a $\mathrm{T}_{2}{ }^{*}$-weighted FLASH sequence [198] with highly anisotropic voxels and compared the cortical depth profiles with the standard isotropic acquisition using EPI. The AVF approach presented in this study marks (at least) an $85 \%$ increase in spatial resolution in the cortical depth direction with fMRI compared to typical sub-millimetre acquisitions and remains relatively unaffected by image distortions due to the FLASH readout while having robust and reliable tSNR. An in-house developed visual cortex coil [197] (see Fig. 3.2) was used in this regard for practical advantages, such as an extended visual field for stimulus presentation and improved temporal signal-to-noise (tSNR) throughout the occipital lobe. A relatively low read-out bandwidth of $60 \mathrm{~Hz} / \mathrm{px}$ was chosen to improve image SNR of the AVF data at the $26 \mathrm{~ms} \mathrm{TE}$ and off-resonance effects can be a concern. It is important to note that dynamic off-resonance effects are mostly dominated by field changes due to movement. However, in the absence of deliberate motion, as was the case with the current study, their effect is a small fraction of the voxel size in the frequency encoding direction [203] and therefore, has negligible impact 
on the results.

Please note that for this study we used a highly anisotropic acquisition $(0.1 \times 1.4 \times 2.0$ $\mathrm{mm}^{3}$ ), whose spatial dimensions can be adjusted if desired. That is, other voxel dimensions, e.g. voxels with $0.3 \times 1.0 \times 1.0 \mathrm{~mm}^{3}$ having approximately a similar voxel volume, can be used with the same MR sequence. Such voxels still enable us to probe laminar fMRI activation without the need for spatial interpolation and at the same time, having the other dimensions only slightly larger than in standard laminar studies. Finally, as the choice of high-resolution direction is up to the investigator, we envision that multiple high-resolution directions can be used and thereby allowing the underlying high-resolution activation pattern to be inferred. In a future study, we will explore this possibility.

The current study relies on $\mathrm{T}_{2}{ }^{*}$-contrast using $\mathrm{GE}$ acquisition and therefore, suffers from the same vascular biases as isotropic GE acquisitions. GE-BOLD signal has been theoretically predicted (see [43, 49] and references therein) to have high sensitivity to local vascular and metabolic changes that are tightly coupled to the regional neuronal activation [101], but its tissue and laminar specificity is reduced due to the inherent bias of the GE-BOLD signal to both ascending and pial draining veins. This has also been consistently observed experimentally by mapping the GE-BOLD signal across cortical depths [66, 67, 89, 94, 95, 133], wherein the GE-BOLD signal increases towards the pial surface, inconsistent with laminar profiles observed using invasive electrophysiology. Several modelling efforts have tried to address the issue of venous bias in the GE-BOLD data in the context of laminar fMRI $[49,109,110]$ but still require validation with invasive electrophysiology.

Approaches, such as SE-BOLD and GRASE-BOLD acquisitions, also suffer from 
the ascending vein effect because of a) direct contribution of ascending veins to the $\mathrm{T}_{2}$-contrast and $\mathrm{b}$ ) an additional $\mathrm{T}_{2}{ }^{*}$ decay during read out [57], albeit to a lesser degree compared with GE-BOLD acquisition [43, 49]. Alternatively, other fMRI techniques, such as VASO (measuring CBV) or ASL (measuring CBF), are promising candidates to study cortical depth profiles without the unidirectional spatial blurring associated with the ascending veins. However, total CBV contrast may be associated with point-spread function extending beyond the locus of neuronal activation due to a) unidirectional spatial blurring in the direction of the penetrating arteries: It has been shown that, for short stimulus duration, the largest CBV change is in the arteries and they can dilate retrogradely in the upper layers with respect to location of neuronal activation; b) significant CBV changes in veins for long stimulus duration (for a recent review, see [43]). Currently, our novel anisotropic approach is limited to GE-BOLD contrast, but future work can expand it to these alternative contrasts.

In both acquisition types investigated in this study (i.e. isotropic vs anisotropic), we confirmed the increase of the BOLD signal amplitude towards the GM-CSF boundary when considering the same ROI. Differences between the acquisitions could be observed at the depths close to the GM-CSF boundary (Fig. 3.5b), possibly due to a) the inherent smoothing of the IVE acquisition, or b) contamination of signal changes due to non-local susceptibility effects stemming from the draining pial veins. With regard to a), the higher signal increase near the pial surface with the IVE acquisition may originate from the larger point-spread function, thereby leading to greater partial voluming with surface veins. In this regard, Monte Carlo simulations were carried out to simulate the effect of leakage of the signal in the pial vein to the cortical layers due to partial volume effects on an anatomicallyinspired cortex [72] by inducing BOLD near the CSF/pial surface with two dif- 
ferent voxel resolutions $(0.75 \mathrm{~mm}$ and $0.125 \mathrm{~mm}$ ) (see Methods, Simulating the large BOLD signal near CSF/pial boundary). The experimentally observed differences in BOLD signal near the CSF/pial surface between the IVE and AVF data (Fig. 3.5b) were reproduced by the simulations (see Fig. 3.8a). Thus, the partial volume effect can be significant across depths, particularly with larger voxel sizes. With regard to b), it is often believed that masking out draining veins from the cortical depth analysis excludes signal contamination from draining veins [68, 77]. However, as the magnetic field disturbances from surface blood vessels extend to the tissue voxels, this results in fMRI signal changes even in remote voxels (i.e. "blooming effect" [170, 204]). For example, this results in apparent broadening of vessel diameter after injection of a contrast agent with different magnetic susceptibility. In high resolution fMRI, therefore, the laminar profiles can potentially be affected by remote pial vessels, which can influence the signal changes in $3 \mathrm{D}$ space.

It is important to note that the magnitude of the blooming effect depends on the spatial resolution of the voxels. Thus, the blooming effect stemming from pial veins within or outside of the imaging slice may be lower or higher in the anisotropic approach due to altered intra-voxel field inhomogeneity. Thus, whether the total blooming effect is stronger in the AVF or IVE depends on the relative location of these veins. In the current study, a lot of care was taken to place the ROI during acquisition guided by the anatomical image in order to have the largest distances to cortical surfaces outside of the imaged volume. Therefore, it is plausible to assume that with such an approach, the blooming effect due to the pial veins is reduced, compatible with our finding of smaller BOLD signal amplitudes in the upper layers of the cortex in the AVF vs. the IVE approach (see Fig. 3b). Nevertheless, a future modelling study on quantitative evaluation of the blooming 
effects stemming from surface veins as a function of distance [205], orientation $[95,177]$ and voxel sizes in the context of depth-dependent GE-BOLD is necessary to provide further insight into this effect.

\subsubsection{Effective spatial resolution of the isotropic acquisition}

The effective spatial resolution of the IVE acquisition was tested using simulations and explored the effect of the number of sampled cortical depths and the ROI size (along cortical distance) on the estimated cortical depth profiles by reconstructing induced PSF (FWHM $\approx 1 / 5$ of cortical thickness in Fig. 3.7) with three (e.g. infragranular, granular, supragranular) and twenty one sampled cortical layers using the standard post-processing by upsampling. The results of the simulations indicate that increasing the number of voxels only minimally improves the inherent blurring of the reconstructed PSF [67]. Additionally, we can conclude that for a given spatial resolution of the functional data (assuming isotropic voxels), an ROI with number of voxels $<100$ is insufficient to achieve a reasonably accurate estimate of PSF. While in practice, it is nearly impossible to reconstruct the 'true' width of the laminar profile with hitherto standard depth sampling approaches, a technique known as 'spatial GLM' [72-74, 161, 183] is presently under development, which accounts for the partial volume distribution between voxels spanning the cortical depths, can in theory, reconstruct the 'true' laminar profile almost perfectly. However, this approach requires high SNR to be reliable [72], which can be challenging at high spatial resolutions. Alternative data analysis approaches using sub-millimetre isotropic data include sorting voxels by the relative distance of their centroids to the cortical boundaries [67, 75]. This specific approach avoids interpolation into a higher spatial resolution but keeps the data in the original space, which may already be sufficient to demonstrate laminar effects of cogni- 
tive processes in some experiments. Therefore, we consider this approach to be an important, albeit under-utilised, exploration tool for high-resolution fMRI data. However, it still suffers from limitations of inferring laminar fMRI activity from an extended ROI, in contrast to single segments in the AVF approach, as voxels from many cortical segments have to be sampled in order to distinguish different depths.

\section{BOLD signal variability along the cortical distance}

In addition, a major assumption made in the analysis of standard high-resolution fMRI data is that the layer-specific fMRI signal within the ROI only varies across cortical depth but not tangentially along the cortical distance. Thus, the AVF approach affords the unique opportunity to also investigate the BOLD variability along the cortical distance. We observed that each cortical distance segment has a strong depth-dependent BOLD response, but these are markedly different between neighbouring segments. This variability could be due to thermal noise, therefore random, or due to susceptibility-induced processes, therefore possibly meaningful. In order to investigate this, we assessed the variability of the positive BOLD signal change over trials by employing a PCA with the hypothesis that, if the tangential variability was due to physiological or susceptibility-induced processes, the first PC has to represent these profiles with high fidelity. We observed that the first PC (Fig. 3.6a, third panel) retains the tangential variability that we observed in individual trials (Fig. 3.6a, first and second panels). Although a full characterization of the nature of this variability is beyond the scope of this study, we show that the assumption of the BOLD signal homogeneity in an ROI, necessary for the isotropic data analysis, may not be accurate (see also Havlicek et al. [72]) and that this tangential variability has physiological or physical origins. In 
other words, there may be considerable amount of tangential variability also in the isotropic acquisition, which however, may be difficult to visualize directly and take into account in the further processing of the data. At this stage, the physiological or physical correlate of the tangential variability cannot be established (except excluding random thermal noise) as, to the best of our knowledge, no electrophysiological study has been performed studying tangential variability at this spatial scale with laminar probes.

\section{Mapping the BOLD signal along cortical distance with AVF acquisition}

Even though the main focus of the current study was the demonstration of the feasibility and superiority of the AVF acquisition for cortical depth analysis, we exploited the flexibility of the AVF acquisition strategy to also test the feasibility of sampling cortical distance with the ultra-high spatial resolution (i.e. 0.1 $\mathrm{mm}$ ) by swapping the phase-encoding and readout directions. We identified the same patch of cortex from data with high-resolution along the cortical depth and compared the BOLD signal magnitudes to the data with high-resolution along cortical distance. We also demonstrate the tangential variability of the fMRI signal along the cortical distance and the signal averages over cortical depth and sampled signal over cortical distance corresponded very well with each other, further supporting the susceptibility origin of the tangential variability observed in the fMRI responses.

\subsection{Limitations}

One limitation of using the AVF approach is the low brain coverage, which in the current study was defined by a single slice through the occipital lobe. Working 


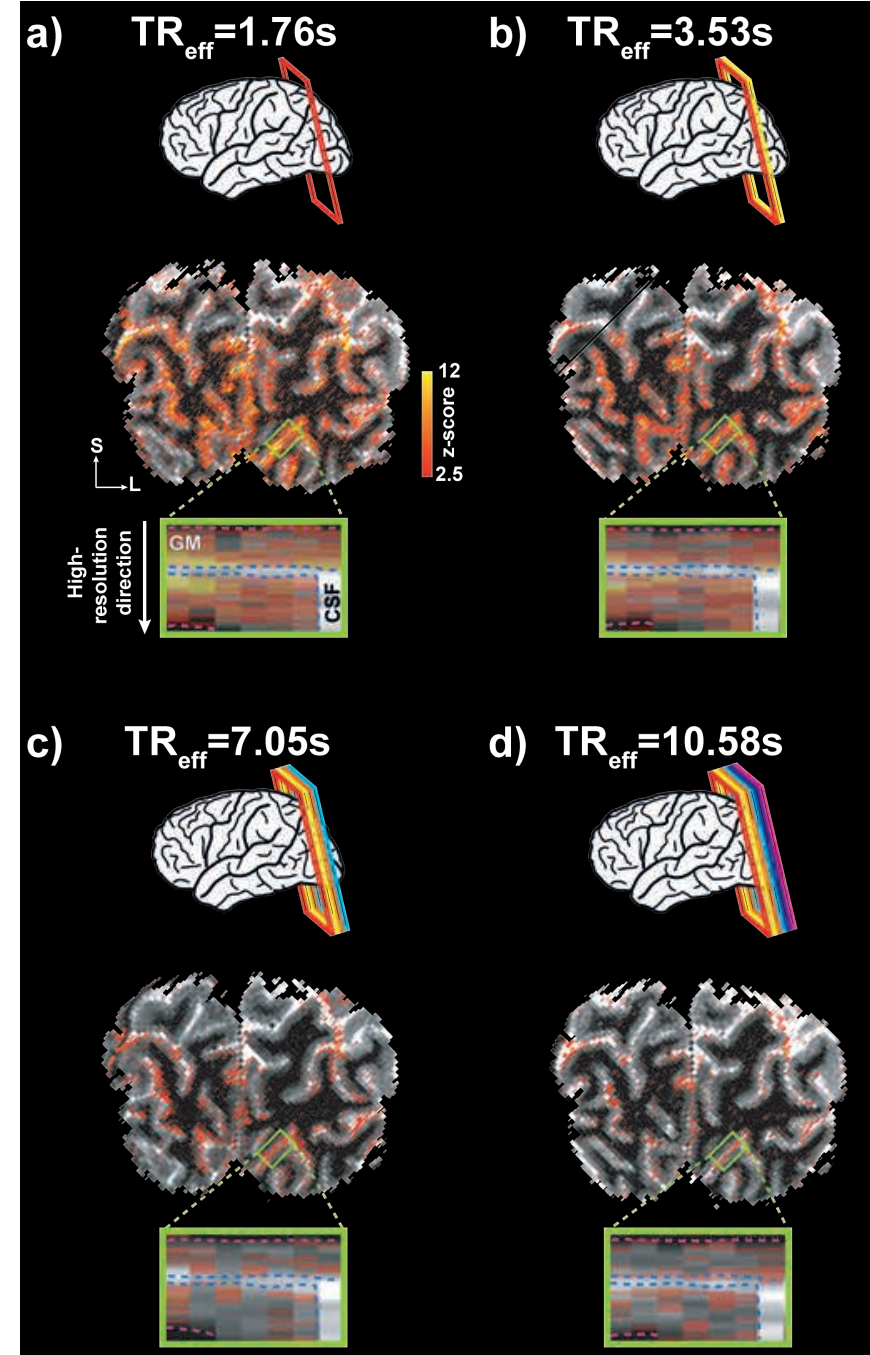

Figure 3.10: Single-subject single-run activation maps overlaid on an MP2RAGE $\mathbf{T}_{1}$ map demonstrating the different acquisition schemes with multiple slices having highest spatial resolution along the laminar direction using the anisotropic 2D-FLASH approach. 
with single slices can be challenging, in particular due to sensitivity to subject motion. However, in the present study, we were able to correct subject motion in all but two runs in total across all subjects and obtained significant activation in all subjects in each motion-corrected run. This was especially aided by the fact that the participants were experienced in high-resolution studies. However, this would not be the case for naïve participants and therefore, future studies employing the AVF approach may consider using prospective motion correction [206] in addition to the manual curation during pre-processing. Please note, that additional slices can be acquired with the AVF approach and thus, extend brain coverage albeit with increasing TR (see Fig. 3.10). Nevertheless, as can be seen with the resulting activation maps, acquiring more than 4 slices is currently not recommended as the tSNR falls rapidly thereafter (or data is acquired for longer durations).

Furthermore, prior knowledge of the subjects' anatomy and the approximate locus of activation are other limitations of the AVF approach, as making informed slice positioning is critical to the accurate sampling of cortical depths. Nevertheless, this information can be obtained using a quick anatomical scan and a functional localizer. Thus, we envision that the AVF approach can be utilized in the following order: (i) Acquisition of low- or high-resolution functional localizer to determine the cortical patch of interest; (ii) Acquisition of high-resolution anatomy to guide the positioning of the anisotropic voxels; (iii) Positioning of the anisotropic slice(s) in order to zoom-in on the cortical depths or distances. We do not suggest using the anisotropic approach in an explorative way without prior knowledge of the activation locus but rather use it to study the intra-cortical processing in a known functional ROI. Insights gained from the AVF approach in this manner can help to interpret the depth-dependent or low-resolution signal changes ob- 
served with isotropic sub- or supra-millimetre acquisitions, typically used in cognitive neuroscience applications. Given that the activation locus is known before the placement of the AVF slice(s), the orientation of the slice(s) can be optimised in a way such that the maximum number of depths or tangential distances is probed.

Lastly, the application of the AVF acquisition approach as presented in the current study is limited to regions with relatively flat patches of cortex due to the anisotropicity. However, even a convoluted cortex is locally flat (i.e. its curvature can be locally approximated by a first order Taylor expansion). Therefore, to be able to apply the AVF method using the same dimensions as in the paper, a relatively flat cortex for about $\approx 1.4 \mathrm{~mm} \times 2 \mathrm{~mm}$ is required, which is reasonably fulfilled in any part of the human brain. Additionally, applying AVF in areas with curvature just effectively reduces the number of cortical depths sampled, which however, may still be sufficient for imaging laminar processing (e.g. instead of $\approx 30$ cortical depths in flat part of the cortex, $\approx 20$ depths may still be discerned with oblique orientations).

The AVF approach is presently only applicable to $\mathrm{T}_{2}{ }^{*}$-dependent studies. Given that GE-BOLD remains the workhorse for most laminar fMRI studies, we believe that the AVF approach can be a useful add-on method to aid interpretability in such studies. In combination with modelling of the non-local BOLD signal due to ascending veins [51], we expect that the AVF approach presented in this study will allow studying functional laminar organization of the human cortex at an unprecedented spatial detail, currently only possible with invasive methods, such as laminar probes used in electrophysiology. 


\section{Acknowledgements}

The study was supported by the Netherlands Organisation for Scientific Research (NWO) VIDI grant to Kâmil Uludağ (452-11-002) and Benedikt A. Poser (016-178-052), who also received partial funding from the NIH (R01MH111444). We thank Laurentius Huber for the implementation of the tr-FOCI inversion pulse in the MI-EPI sequence, Jonathan Polimeni for the discussion and feedback during the Brain-indepth symposium (BIDS) 2018, and the anonymous reviewers for the critical reading and review of the manuscript. 


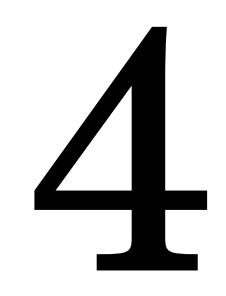

Laminar fMRI using arterial spin labelling

Associated abstract: S. Kashyap, D. Ivanov, M Havlicek, B. A. Poser, and K. Uludağ. "Laminar CBF and BOLD fMRI in the human visual cortex using arterial spin labelling at 7 T". in: Proceedings of the 27th Annual Meeting of ISMRM. Montréal, Canada, 2019. 



\section{Abstract}

Laminar fMRI at ultra-high magnetic field strength is typically carried out using the BOLD contrast. Despite its unrivalled sensitivity to detecting activation, the BOLD contrast is limited in its spatial specificity (e.g. due to intra-cortical ascending and pial veins). Alternatively, regional changes in cerebral blood flow (CBF) are a quantitative measure directly related to neuronal activation, both spatially and temporally. Arterial spin labelling (ASL) can be used to simultaneously acquire both $\mathrm{CBF}$ and BOLD contrasts for fMRI. However, ASL for laminar imaging is challenging due to the lower SNR of the perfusion signal and higher power deposition (SAR). In the present study, we acquire, for the first time, in humans isotropic sub-millimetre spatial resolution in CBF using 3D-EPI PASL at 7T. We show that robust statistical activation maps can be obtained with CBF in a singlesession. We observed the characteristic BOLD signal increase to the superficial laminae, whereas the $\mathrm{CBF}$ profile shows a decrease of the amplitude towards the cortical surface. Using model-based spatial convolution, we assess the apparent discrepancy between the CBF and BOLD profiles and show that, taking into account the draining vein effect on the BOLD signal, the empirically measured CBF and BOLD profiles are in fact consistent with each other. This study demonstrates that caution has to be exercised when interpreting BOLD signal laminar profiles as direct representation of the cortical distribution of neuronal activity and that the measured $\mathrm{CBF}$ profiles can be used to validate models of the draining vein effect on the GE-BOLD signal. 



\subsection{Introduction}

Neuronal activity in the brain is associated with an increased metabolic demand accompanied by changes in haemodynamics such as blood oxygenation, flow and volume (for reviews see, [49, 208, 209]). Functional magnetic resonance imaging (fMRI) is a technique that can non-invasively measure these changes and allows to infer the spatial pattern of neuronal activity while performing a task or at rest. Improvements in MRI technology over the past decades such as, higher-field strengths, parallel imaging, novel sequences and optimised pulse designs have pushed the spatial and temporal limits to an extent wherein MRI at ultra-high field (UHF, $\geq 7 \mathrm{~T}$ ) can routinely achieve sub-millimetre spatial resolution voxels for both structural and functional imaging with short acquisition times [54, 55, 194, 210-212]. While the earliest fMRI investigations yielded robust, reproducible functional parcellation of different brain areas [213-217] consistent with previous ex vivo cyto- and myelo-architectural studies [58, 59], the advantages of UHF fMRI have enabled cognitive neuroscientists to investigate the circuitry within areas across cortical depths and columns in humans [193, 218]. A vast majority of standard and laminar fMRI studies have been performed using the Blood Oxygenation Level-Dependent (BOLD) contrast [35, 38]. While the BOLD contrast excels in its sensitivity to detect signal changes [80] even at sub-millimetre spatial resolutions due to its robust signal-to-noise (SNR), it is inherently limited in its spatial specificity relative to site of neuronal activation due to strong signal bias that stems from deoxy-haemoglobin drainage through intra-cortical ascending veins [51] and the non-local signal spread (blooming effect) through pial veins [219, 220] (for a recent review, see Uludag and colleagues [49]). Studies investigating the specificity of the laminar BOLD response in humans $[66,77,82,94$, 95, 98, 184, 195] and animals [111, 133, 221-223] have consistently observed a 
peak signal change in BOLD at the superficial layers and pial surface despite the fact that the largest neuronal activity is in the input layers (layer IV in human V1) for feed-forward stimuli $[68,195]$. In recent years, several attempts have been made to develop steady-state [110] and dynamic models [51, 109] of the laminar BOLD response that can allow model-driven deconvolution of the laminar BOLD profiles [195]. However, such model-driven approaches still require systematic experimental validation. Another limitation of the BOLD signal is that it is not a quantitative measure associated with neuronal activity. The BOLD signal amplitude is affected by heterogeneous baseline parameters such as blood volume [43], and therefore, can be highly variable across participants and can also vary within the same participant over different sessions.

However, the versatility of MRI provides means to alternatively measure other (non-BOLD) haemodynamic reponse parameters such as cerebral blood volume (CBV) using vascular space occupany (VASO) [81, 224, 225] or cerebral blood flow (CBF) using arterial spin labelling (ASL) [226, 227]. Most studies using these non-BOLD approaches have been carried out in animal models [85, 133, 222, 223, 228-230] and has only been applied to high-resolution human studies with the advent of UHF fMRI [80]. Among the two most promising candidates, CBV-weighted imaging using VASO has seen a resurgence for laminar fMRI applications [82] but $\mathrm{CBF}$-weighted imaging using ASL has been mostly limited to relatively low spatial resolution $(\approx 3-4 \mathrm{~mm})$ studies. From the perspective of laminar fMRI, $\mathrm{CBF}$ is a highly desirable contrast, even more so than total CBV $[49,80]$, due to its spatial and temporal proximity to neuronal activation, that is, changes in CBF are spatially and quantitatively better correlated to neuronal activity [86, 222]. CBF fMRI [231-234] using ASL is, however, limited by the relatively lower SNR of the perfusion-weighted signal (due to lower microvascular density), lower 
temporal resolution due to the post-labelling delay (PLD) (required to allow the labelled blood to reach the imaging slab) in the ASL acquisition scheme (for a complete overview of ASL methodology see the white paper by Alsop and colleagues [235]), and higher power deposition (SAR) making it difficult to achieve high spatial resolutions and, consequently limiting its application to laminar fMRI [80]. Going to UHF can have several advantages for CBF fMRI, for instance, the sheer gain in SNR due to increased field strength [53, 84], and the increased $\mathrm{T}_{1}$ [236, 237] allowing longer PLDs, thereby, improving the perfusion SNR and obtaining larger brain coverage [84]. Taking together these advantages at UHF, the spatial specificity of the $\mathrm{CBF}$ in response to neuronal activation and the fact that ASL acquires both BOLD and CBF-weighted images simultaneously makes it a very attractive tool for laminar fMRI. Recent developments using ASL at 7 $\mathrm{T}[84,88]$ have enabled pushing the spatial resolution for CBF-mapping to the sub-millimetre regime [238] by overcoming several technical challenges that included optimisation of sequence and pulse design [180], using dielectric pads in order to improve the labelling efficiency [84], and utilisation of a 3D-EPI readout [141]. Recently, Ivanov and colleagues [238] demonstrated the feasibility of whole-brain ASL in humans at $7 \mathrm{~T}$ at sub-millimetre resolution to measure and quantify baseline CBF. In the present study, we build on our previous work to acquire, for the first time, sub-millimetre resolution simultaneous $\mathrm{CBF}$ and BOLD fMRI in the human visual cortex at $7 \mathrm{~T}$. By reducing the partial-voluming using sub-millimetre voxels, we study the spatial localisation of the CBF and BOLD responses within the cortical ribbon and demonstrate that robust, single-subject, single-session, functional CBF maps can be obtained for laminar fMRI in humans at $7 \mathrm{~T}$. Finally, we investigate the cortical depth-dependence of CBF and BOLD signals in response to visual stimulation in humans. 


\subsection{Methods}

\subsubsection{Participants}

Six healthy volunteers (median age=28 years, 2 female) participated in the study following screening and having given written informed consent. The study was approved by the Ethics Review Committee for Psychology and Neuroscience (ERCPN) at Maastricht University and all procedures followed the principles expressed in the Declaration of Helsinki.

\subsubsection{Data acquisition}

Data were acquired on a whole-body Siemens Magnetom 7T research scanner with a gradient system capable of maximum gradient amplitude of $70 \mathrm{mT} / \mathrm{m}$ and maximum slew rate of $200 \mathrm{~T} / \mathrm{m} / \mathrm{s}$ (Siemens Healthineers, Erlangen, Germany) and a 32-channel receive phased array head coil (Nova Medical, USA). The participant placement and preparatory procedure followed the protocol previously described in $[84,88]$, in short, the eye centres were taken as isocentre reference instead of the eyebrows as is typically done and the cushions were added as required under the neck of the participants so as to ensure that the large feeding arteries to the brain were parallel to the $\mathrm{B}_{0}$. In addition, two $18 \times 18 \times 0.5 \mathrm{~cm}^{3}$ high-permittivity dielectric pads [239] containing a 2.8:1 solution of calcium titanate $\left(\mathrm{CaTiO}_{3}\right)$ and heavy water $\left(\mathrm{D}_{2} \mathrm{O}\right)$ by weight [240] were placed on either side of the head at the level of the participant's temporal lobes to increase $B_{1}$ efficiency (therefore, labelling) at $7 \mathrm{~T}[241]$.

Stimulus paradigm: Full contrast black-and-white radial flickering $(\approx 8 \mathrm{~Hz})$ checkerboard was presented using PsychoPy [136] for $20 \mathrm{~s}$ (stimulus on) followed by $40 \mathrm{~s}$ of an iso-luminant grey background (stimulus off). Each functional run 
lasted $\approx 12$ minutes consisting of a $30 \mathrm{~s}$ initial baseline period and ten stimulus on-off blocks. The participants were instructed to remain motionless and fixate on a central fixation dot throughout each functional run.

Anatomical: Anatomical data was acquired using a 3D-MP2RAGE [117] at 0.9 $\mathrm{mm}$ isotropic spatial resolution(192 sagittal slices; GRAPPA $=3 ; \mathrm{FoV}_{\text {read }}=230$ $\mathrm{mm} ;$ phase-encoding $=\mathrm{A} \gg \mathrm{P} ; \mathrm{TI}_{1} / \mathrm{TI}_{2}=900 / 2750 \mathrm{~ms} ; \alpha_{1} / \alpha_{2}=5^{\circ} / 3^{\circ} ; \mathrm{TE} / \mathrm{TR}=$ $2.39 / 4500 \mathrm{~ms} ;$ partial-Fourier ${ }_{\text {phase }}=6 / 8$; bandwidth $=250 \mathrm{~Hz} / \mathrm{px}$; echo-spacing $=$ $6.6 \mathrm{~ms})$.

Functional: Functional data were also acquired at $0.9 \mathrm{~mm}$ isotropic resolution using a pulsed ASL (PASL) sequence [242] with a 3D-EPI readout [141] employing a FAIR QUIPSS II labelling scheme [243, 244] (44 axial slices; GRAPPA = 4; $\mathrm{FoV}_{\text {read }}=192 \mathrm{~mm}$; phase-encoding $=\mathrm{A} \gg \mathrm{P} ; \mathrm{TE} / \mathrm{TR}=15 / 2850 \mathrm{~ms} ; \alpha=19^{\circ} ; \mathrm{TI}_{1} / \mathrm{TI}_{2}$ $=700 / 1891 \mathrm{~ms} ;$ partial-Fourier phase $=5 / 8 ;{\text { partial }- \text { Fourier }_{\text {slice }}=7 / 8 ; \text { Ref. }_{\text {lines }} P E}$ $=64 ;$ Ref. scan mode $=$ FLASH; bandwidth $=1124 \mathrm{~Hz} / \mathrm{px} ;$ echo-spacing $=1.02$ $\mathrm{ms})$. Immediately after each of the four functional runs, five volumes with opposite phase-encoding were acquired for distortion-correction.

\subsubsection{Data processing}

The anatomical data was pre-processed in SPM12 [152]. The second inversion image of the MP2RAGE was subjected to the automated segmentation in SPM12 [145]. A brain mask created using FSL BET [245] was combined with the thresholded non-brain tissue components of the SPM12 segmentation to mask out the non-brain tissue and large sinuses (for step-wise procedure see Fig. 4.1). The $\mathrm{T}_{1}$-w MP2RAGE image was bias-corrected using SPM12 and the combined brain mask was then applied before piping the pre-processed $\mathrm{T}_{1}-\mathrm{w}$ MP2RAGE as input to Freesurfer v.6.0 (recon-all) [246]. The segmentation and surface construction was 
done in the native resolution and the segmentation quality in the occipital lobe was curated manually using freeview. An additional CSF mask was created from quantitative $\mathrm{T}_{1}$ image of the MP2RAGE using the high-intensity voxels $(\geq 3500$ a.u.). A probabilistic retinotopic atlas by Wang and colleagues [247] was applied to the Freesurfer reconstructed data using a docker image (nben/occipital_atlas) [248] so as to obtain separate dorsal and ventral V1 and V2 ROIs in subject-specific space. The dorsal and ventral labels of V1 and V2 thus obtained were combined to form V1 and V2 ROIs used in the rest of this study. The functional data were subjected to affine motion-correction by realigning each time-series volume to the temporal mean of each run (antsRegistration) followed by distortion-correction using the temporal means of the functional and opposite phase-encoding data to find a middle-ground undistorted template (antsMultivariateTemplateConstruction) which was followed by co-registration of the undistorted template to the anatomical $\mathrm{T}_{1}-\mathrm{w}$ MP2RAGE data (antsRegistration) using Advanced Normalisation Tools (ANTs) [200]. In the ANTs framework, all transforms were concatenated and applied to the functional time-series data in a single resampling step using a $4^{\text {th }}$ degree B-Spline (antsApplyTransforms, Fig. 4.2). Statistical analyses were carried out using FSL FEAT [148, 249] by modelling three regressors i.e, the stimulus design convolved with the canonical haemodynamic response function (HRF) representing the BOLD signal, the alternating label-control acquisition of the ASL sequence representing the baseline CBF and the combination of these two regressors representing the CBF activation. Following Freesurfer's segmentation and reconstruction, nine intermediate equi-distant GM surfaces were created in Freesurfer [66, 75, 250] (i.e, a total of eleven laminar surfaces including pial and white) and we additionally extended the surfaces into CSF and WM. Laminar analyses were carried out in surface space by sampling the functional time-series 

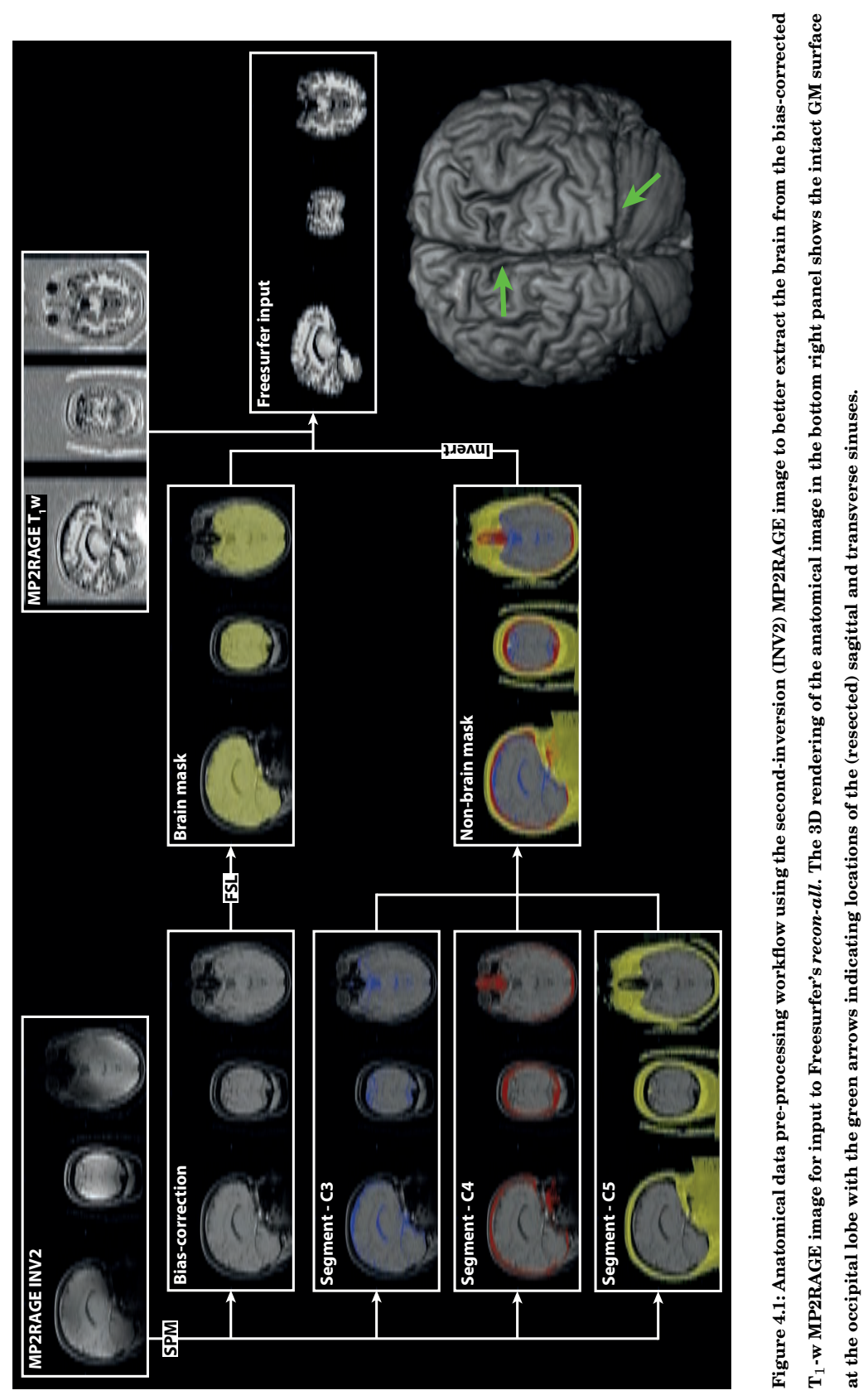


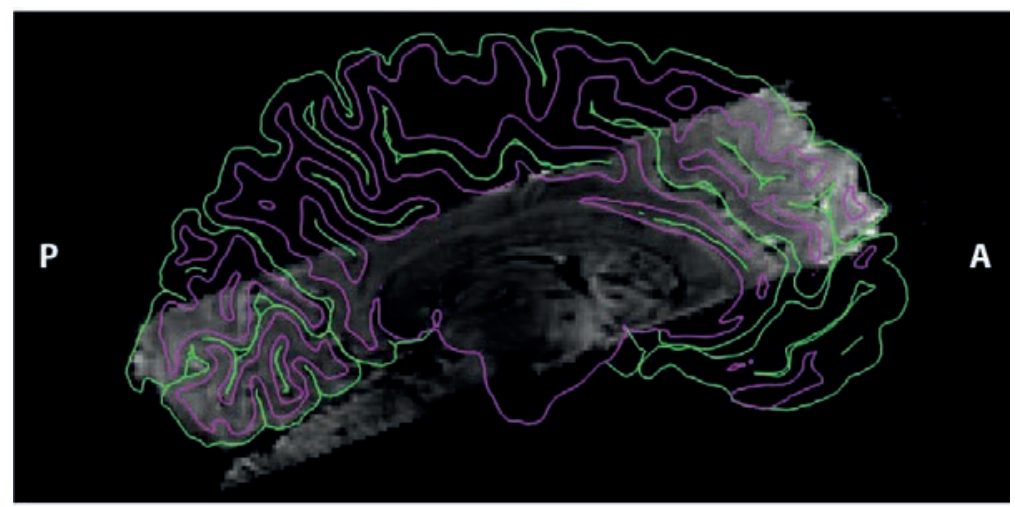

Figure 4.2: Example of the result of the single step resampling of the functional data (combining motion, distortion-correction and co-registration to anatomical image) applied using the ANTs framework. The temporal mean functional image (mid-sagittal view showing the oblique coronal acquisition) is overlaid with the segmented anatomical boundaries from Freesufer. The pink and green lines indicate the WM-GM and GM-CSF boundaries respectively.

signal from the ROIs using nearest-neighbour interpolation. These laminar timecourses were imported into MATLAB R2016b to obtain the BOLD and CBF timecourses by surround-averaging and surround-subtracting, respectively [251]. The BOLD time-series were then rescaled to compute the percent BOLD signal change relative to the pre-stimulus baseline period $(\approx 0-10 \mathrm{~s}$ before stimulus onset) and the $\mathrm{CBF}$ time-series was normalised by the temporal mean signal (as a proxy for $\mathrm{M}_{0}$ ) so as to calculate the absolute change in CBF (in arbitrary units but its translation to physiological units is but a scaling factor). Laminar profiles of BOLD and $\mathrm{CBF}$ were calculated by averaging the time-points corresponding to $\approx 14-28 \mathrm{~s}$ following stimulus onset. In addition, the baseline CBF laminar profile was calculated using simple subtraction of the label-control time-points during the baseline period $(\approx 0-30 \mathrm{~s}$ at the beginning of the run) and pre-stimulus intervals $(\approx 0-10 \mathrm{~s}$ before stimulus onset) of the stimulus blocks across all runs of each participant. 


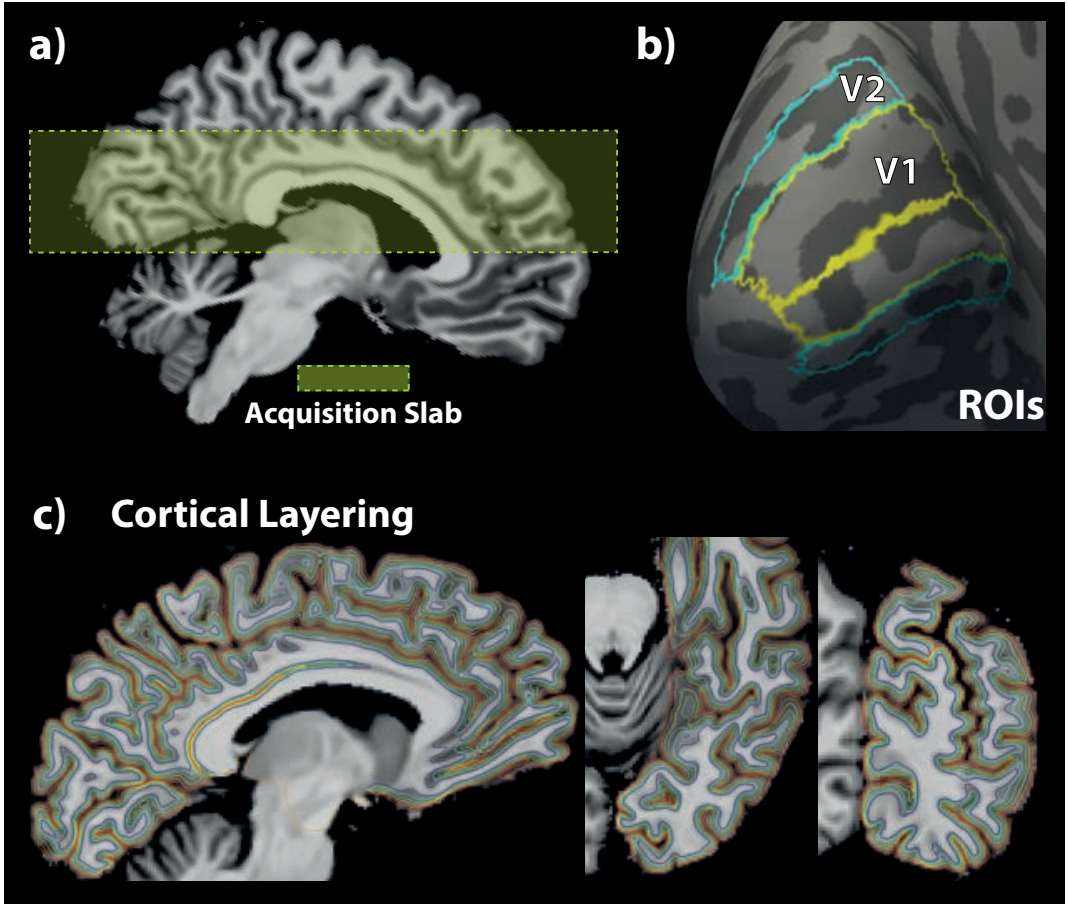

Figure 4.3: (a) Illustration of the coverage obtained by acquisition slab used in the present study overlaid on a $T_{1}$-w mid-sagittal slice of an example participant. (b) Contours of the V1 and V2 labels obtained from the Wang [247] probabilistic atlas overlaid on an angulated posterior-medial view of the left inflated hemisphere of the an example participant. (c) Sagittal, axial and coronal views of the equi-distant laminar surfaces overlaid on the anatomical $\mathbf{T}_{1}$-w image of the same participant.

\subsection{Results}

In the present study, the data acquisition slab was oriented so as to cover the entirety of the occipital lobe (Fig. 4.3a) in all participants. 


\subsubsection{Functional activation analysis}

Robust statistical activation was obtained for all participants for both the BOLD signal and CBF (Fig. 4.4a-b, colour map overlaid on inflated surface and volume space). For illustrative purposes, single-subject event-related average raw ASL signal time-courses are shown in Figure 4.4a-b, sampled from the statistically significant $\left(\mathrm{p}_{F D R}<0.05\right)$ BOLD and CBF voxels in the atlas-based V1 and V2 delineations (Fig. 4.3b). Please note that the envelope of the ASL time-courses represents the BOLD signal and the modulation depth (i.e. zig-zag difference) represents the amount of labelled spins delivered to the tissue i.e. proportional to the $\mathrm{CBF}$. The dynamic ranges of the control-label pairs are visibly different between the time-courses sampled from the statistically-significant BOLD and $\mathrm{CBF}$ voxels (Fig. 4.4a-b). The time-courses for CBF activation show larger difference between pairs of data points (i.e. the zig-zag modulation) indicating that the labelling was robust and that observed responses are capturing the blood flow changes over time, whereas these zig-zag changes relative to the overall signal envelope in the time-courses for BOLD activation, are captured to a much lesser extent. The baseline blood flow response (estimated using FSL FEAT) was also robust in every participant and results from an example participant are shown in Figure 4.4c, demonstrating the difference in $\mathrm{CBF}$ distribution between white and grey matter. Additionally, the event-related average ASL time-course from sagittal sinus voxels close to V1 is shown in Figure $4.4 \mathrm{~d}$ and does not exhibit much influence of the tagging. We also observed that the BOLD and CBF activation estimated from FSL FEAT in response to the stimulus differed in terms of their spatial spread of activation and this spatial non-overlap is illustrated in Figure 4.6. It can be seen that the larger z-scores of the BOLD response are localised close to the pial/GM-CSF surface as is expected with GE fMRI. On the other hand, 
$\mathrm{CBF}$ responses are better localised to the cortical grey matter and not present in regions encapsulated by the yellow boxes.

\subsubsection{Laminar analysis}

The group-average laminar time-courses of BOLD and absolute CBF sampled from V1 and V2 are shown in Figure 4.7. We observed inter-regional differences with laminar responses of both the BOLD signal and CBF, with V2 having a lower amplitude than V1 in both measures. While we reproduce the characteristic increase in BOLD signal amplitude towards the pial surface compared to the deeper laminae $[66,68,89,94]$, the CBF responses exhibit the opposite behaviour with the largest absolute CBF changes in the deeper laminae. We found a strong poststimulus undershoot (PSU) in the BOLD $[89,134,153]$ and also detected a PSU in the CBF albeit relatively smaller than the BOLD PSU. In addition, the CBF shows slight oscillatory behaviour in the post-stimulus period. Laminar BOLD and CBF profiles (positive responses i.e. mean of time segment 18-28 s) are plotted in Figure 4.7 together with the laminar profile of the baseline absolute CBF which was obtained by a simple subtraction of labels and controls from the baseline portion of the functional scan. We estimated the slopes of the laminar profiles obtained from $\mathrm{CBF}$ and BOLD using a linear regression analysis. As is evident from Figure 4.6, the BOLD profiles exhibit a positive slope (V1: $0.25 \pm 0.04, \mathrm{~V} 2: 0.13 \pm 0.03)$ compared to the CBF profiles which exhibit a negative slope (V1: $-0.55 \pm 0.09$, V2: $-0.44 \pm 0.08$ ). Upon comparing the slopes, it is no surprise that the differences between the CBF and BOLD profiles are statistically significant $\left(\mathrm{V} 1: \mathrm{F}_{(1,128)}=\right.$ $\left.63.61, \mathrm{p}<0.0001, \mathrm{~V} 2: \mathrm{F}_{(1,128)}=47.01, \mathrm{p}<0.0001\right)$. 


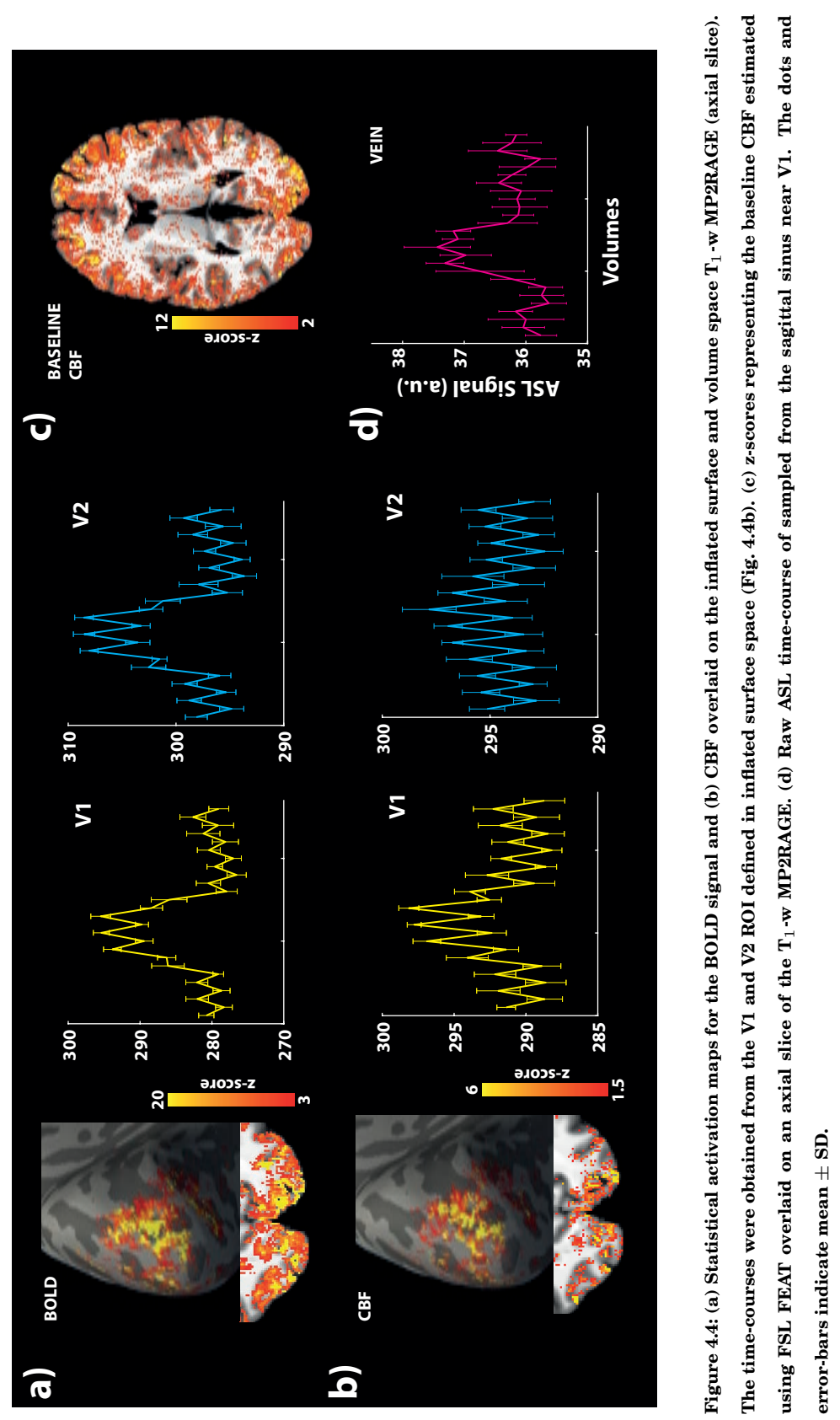




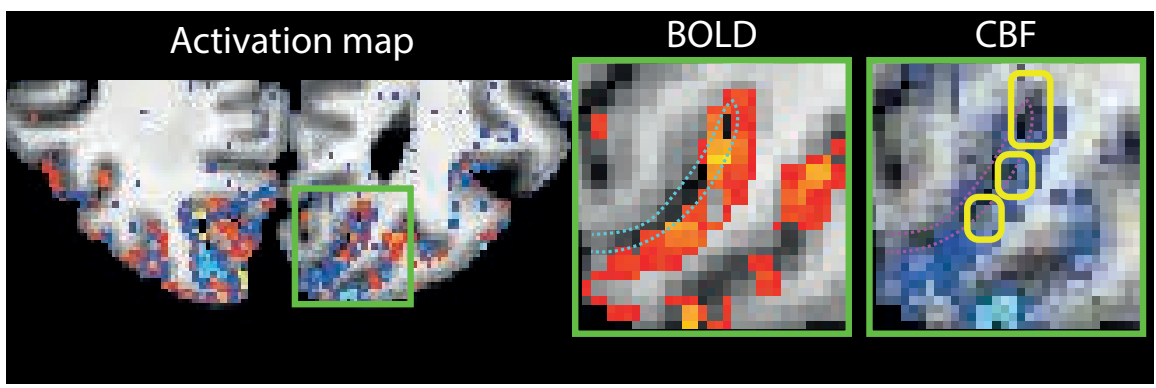

Figure 4.5: Left-to-right: Statistical activation map for BOLD (red-yellow) and CBF (blue-cyan) overlaid on a $\mathbf{T}_{1}$-w axial slice of the occipital lobe. The green box represents the zoomed-out views in the next two panels. The cyan and magenta dotted lines indicate the pial boundary. The yellow boxes indicate regions where BOLD activation is present but not CBF.

a)

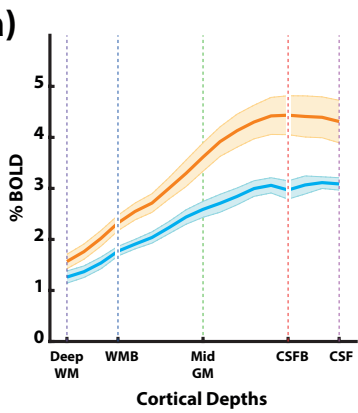

b)

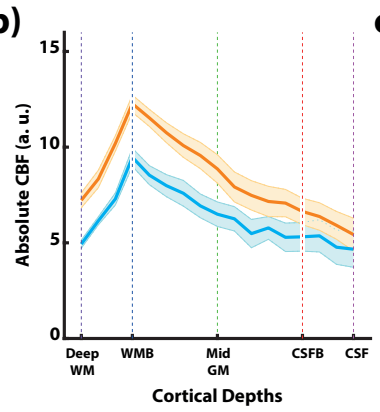

c)

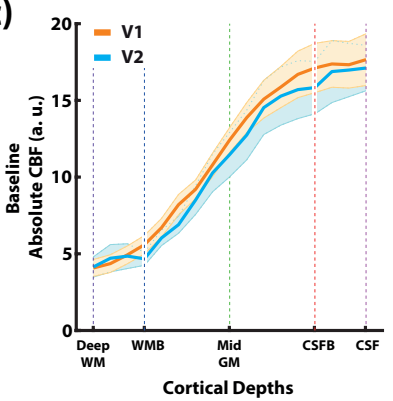

Figure 4.6: Group average laminar profiles of the (a) \%BOLD, (b) Absolute CBF and (c) Baseline absolute CBF. The coloured solid lines and shaded regions indicate mean \pm SEM. 

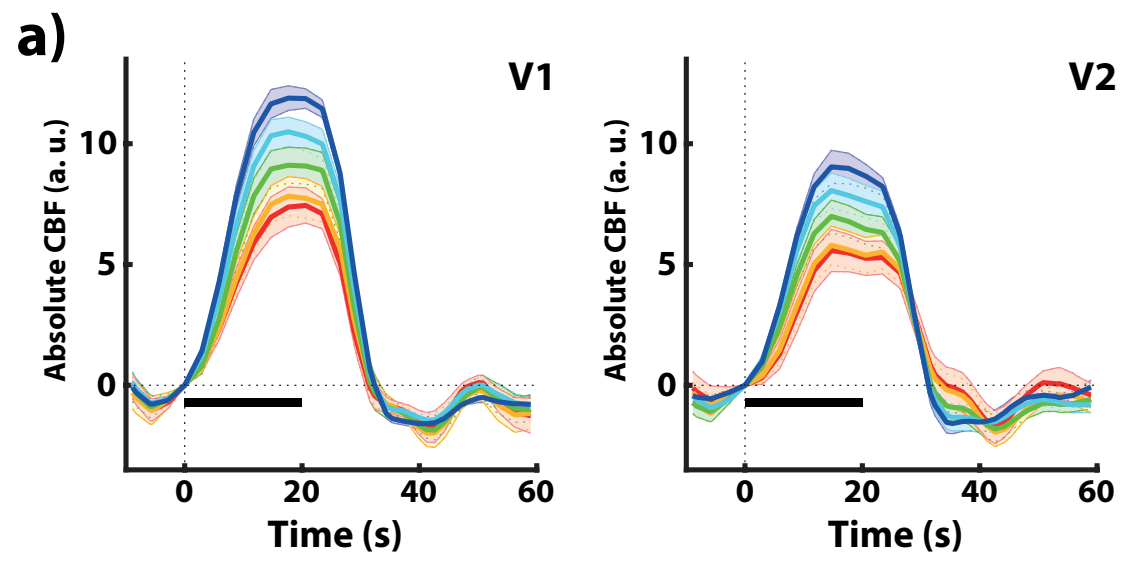

b)
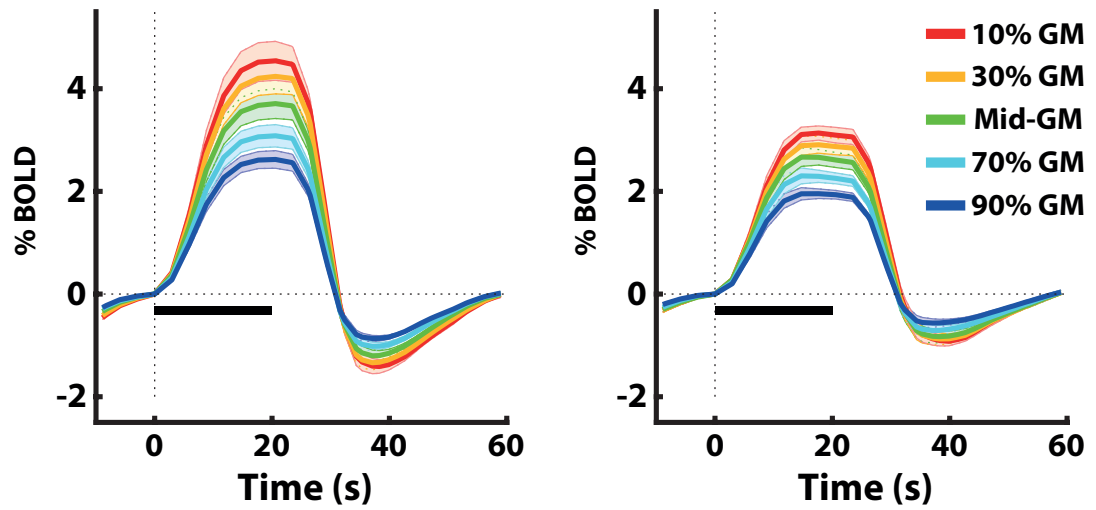

Figure 4.7: Group average time-courses sampled from V1 and V2 for five intermediate GM laminae (out of a total of nine in GM, one pial and one WM) shown for (a) BOLD and (b) CBF. The black bar indicates the stimulus duration. The coloured solid lines and shaded regions indicate mean \pm SEM. 


\subsection{Discussion}

Here, we demonstrated for the first time, simultaneously acquired CBF and BOLD for laminar fMRI in the human visual cortex using pulsed ASL at $7 \mathrm{~T}$ with isotropic sub-millimetre spatial resolution. It is important to note that we acquired the largest spatial coverage possible (to date) covering almost the entire occipital lobe (Fig. 4.3a) while ensuring optimum labelling was feasible at this resolution. The sequence parameters were optimised in previous pilot studies.

\subsubsection{Data processing}

We developed a novel workflow to pre-process anatomical images (Fig. 4.1) by taking advantage of the second inversion time image MP2RAGE to automatically mask the large sagittal and transverse sinuses that enable more accurate pial surface delineation using Freesurfer. Please note that in some subjects the workflow required manual corrections of the segmentation masks albeit very little. Upon completion of the Freesurfer recon-all, we used a docker image [248] that uses a probabilistic retinotopic atlas developed by Wang and colleagues [247] in Freesurfer to generate labels of V1 and V2 in subject-space (Fig. 4.3b). We tested this atlas-based approach on separately acquired retinotopic dataset [195] acquired using the same scanner and head coil, therefore, same visual field, in order to verify that the algorithm was performing as expected (data not shown). Cortical layering was done in Freesurfer using equi-distant approach [66, 75] as equi-volume layering is not natively supported in Freesurfer. Recently, however, an open-source toolbox, Surface Tools [252], became available that performs equivolume layering for Freesurfer processed anatomical data and is promising for use in future studies. Nevertheless, for spatial resolutions such as the present study the exact choice of the layering model does not affect our main conclusions 
$[76,250]$. Despite the fact that the layering is done on the whole cortical ribbon (Fig. 4.3c), we manually ensured that the delineations were accurate within the V1 and V2 masks in each subject. The functional data was also processed in a novel workflow using the ANTs framework [199, 200] in order to concatenate transformations of motion, distortion-correction and co-registration to the anatomical image in a single resampling step. While this has been highly desired for minimal processing of functional data, as far as we know, it has not been demonstrated for laminar fMRI. Previously [89], we have encouraged studies to align anatomical-to-functional data instead of the conventional functionalto-anatomical data in order to reduce the blurring due to applying several resampling steps on the high-resolution high-fidelity functional datasets. Due to our reliance on the retinotopic delineation in surface space and partial coverage functional acquisition, we could not afford to do this. However, we overcame this limitation by combining all the transformations into using a single resampling step. Thereby, we are less prone to multiple-resampling-related blurring that is associated with conventional data processing strategies. Nevertheless, we are exploring the possibility to adapt Freesurfer or alternatives such as Nighres to future partial coverage sub-millimetre datasets for laminar analysis.

\subsubsection{Functional CBF and BOLD activation}

While we obtained the expected widespread activation in the visual cortex for the BOLD response (Fig. 4.4a), we obtained robust single-subject, single-session activation maps for CBF (Fig. 4.4b) and the baseline CBF response (estimated using a GLM) also shows well-defined localisation to the cortical ribbon (Fig. 4.4c). We also show that the voxels active for functional CBF changes are more spatially localised to cortical GM than the voxels active for the BOLD signal [222] which are 
more localised to the superficial layers and CSF (Fig. 4.5), further demonstrating the better specificity of the CBF signal compared to the BOLD signal as the CBF signal is unaffected by the venous compartments, both pial and ascending veins. The reason is that the tagged arterial water is mostly exchanged with the tissue at the level of the capillaries. In addition, the blood transit time within tissue is on the order of $\approx 1-1.5 \mathrm{~s}$ [253] which together with the transit delay for the labelled blood to arrive at the region-of-interest (in this case, occipital lobe) can be $\approx 1-1.3 \mathrm{~s}$ [226] leads to low remaining longitudinal magnetisation of the tag (due to $\mathrm{T}_{1}$ decay). Consistent with these arguments, we show that the ASL-like zig-zag response (due to the magnetisation differences between label and control) is absent in the veins by sampling voxels from the sagittal sinus close to V1 which has a smooth, temporally shifted haemodynamic response (Fig. 4.8) consistent with the expectation that the magnetisation is fully decayed in the venous blood and therefore, both control and labelled images are similar.

\subsubsection{Laminar CBF and BOLD responses}

We replicated previous findings that the event-related average BOLD signal (Fig. 4.7a) increases towards the superficial layers $[66,89,94,207]$, whereas the absolute CBF change (Fig. 4.7b) exhibits a decrease towards the superficial layers in both V1 and V2. The laminar profiles for BOLD and the absolute CBF are shown in (Fig. 4.6a-b). The resting baseline CBF profile (Fig. 4.6c) shows an increase towards the superficial layers in both V1 and V2. The BOLD signal increase to the superficial layers is better understood and can be attributed to two signal biases stemming from the drainage of deoxy-Hb from deeper to superficial layers through the intra-cortical ascending veins and the non-local blooming effect (discussed in Chapter 3) from the pial veins resulting in large signal changes. The 
presence of these signal biases implies that care must be taken while interpreting the spatial profile of the BOLD signal particularly in the superficial layers [181] and requires well-controlled experimental designs or model-driven spatial deconvolution approaches $[51,195]$ to uncover the underlying laminar neuronal response.The relative increase in the absolute $\mathrm{CBF}$ in the middle to deeper layers is consistent with animal literature [222, 254, 255] and corresponds well with histological studies of vascular architecture [61]. In order to test whether the apparent discrepancy between the BOLD and CBF laminar profiles can be reconciled, we simulated a BOLD profile (Fig. 4.8) from the absolute CBF profile by modelling the effect of the ascending veins. We applied the approach described in [195] but in a forward manner using the $\mathrm{CBF}$ profile as a representation of the local BOLD signal profile, i.e. introduce the ascending vein effect to the CBF profile based on the steady-state laminar haemodynamic model [110]. The simulated profile exhibits the characteristic positive BOLD signal increase towards the surface (similar to Fig. 4.6a) indicating that the measured laminar CBF and BOLD profiles, despite their contrasting depth-dependent behaviours are in fact consistent. Additionally, the BOLD time-courses exhibit a strong post-stimulus undershoot consistent with previous studies $[89,153]$ whereas the CBF time-courses show little or no undershoot with a slightly oscillatory behaviour in the post-stimulus region. These post-stimulus oscillatory transients are consistent with observations in rodent optical imaging [256]. These oscillatory transients have also been observed in human CBF measurements [135] although at much lower spatial resolution than the present study $\left(2.65 \times 2.65 \times 5 \mathrm{~mm}^{3}\right)$ and therefore, any depth-dependent differences would be unresolvable. With our spatial resolutions, it is interesting that we are able to observe depth-dependent differences in post-stimulus haemodynamics. For instance, the post-stimulus CBF oscillations near the WM boundary is much 


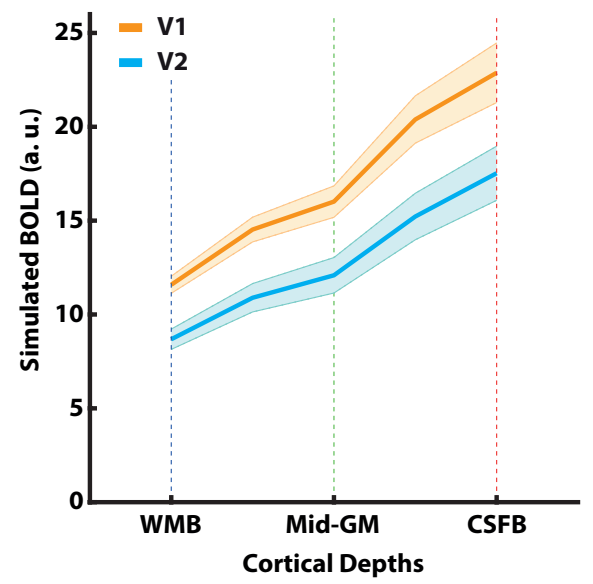

Figure 4.8: Simulated BOLD profile from the absolute CBF profile (Fig. 4.6b) after introducing the ascending vein effect using weights from [195] derived from a steady-state laminar haemodynamic model [110].

smoother and evolves with a different oscillatory phase than the superficial layers where the oscillations are more pronounced ( $4.7 \mathrm{a})$. While the variations of vascular architecture across cortical depths [61] could explain these differences to some extent, pin-pointing the exact arterial physiology that elicits this behaviour is beyond the scope of this study. Taken together, we believe that the current study presents a breakthrough in non-BOLD fMRI research with the development of sub-millimetre resolution simultaneous CBF and BOLD fMRI using ASL and its feasibility for layer-specific investigations which has hitherto been an uncharted territory in humans.

\subsection{Limitations}

Acquisition of artefact- and motion-free $\mathbf{M}_{0}$ scans are crucial for reliable quantification of CBF. Due to the long TR $(\approx 20 \mathrm{~s})$, the $\mathrm{M}_{0}$ scans in all but two subjects had motion-artefacts and aliasing that rendered them difficult to utilise for quan- 
tification. A potential solution for acquiring high-resolution $\mathrm{M}_{0}$ scans would be to instruct the participants to stay still and fixate on a displayed fixation cross and secondly, to acquire multiple $\mathrm{M}_{0}$ scans throughout the session. Lastly, the lowest affordable TE in the present study was $15 \mathrm{~ms}$ owing to the EPI readout which is not ideally suited for perfusion imaging. Although, it would be desirable to achieve short TEs (e.g. $3 \mathrm{~ms}$ ) for better CBF-weighting, it is currently not possible to do so using EPI. To this end, there has been recent progress in non-Cartesian (e.g. spiral readouts) ASL fMRI at ultra-high field [257]. Dual-echo spiral acquisitions can be particularly useful for simultaneous CBF and BOLD imaging achieving the first echo at $\approx 2 \mathrm{~ms}(\mathrm{CBF})$ and the second echo at $\approx 25 \mathrm{~ms}(\mathrm{BOLD})$. The non-Cartesian acquisitions are prone to inaccuracies in the spiral trajectories due to gradient imperfections that require real-time monitoring and correction using specialised magnetic field-monitoring cameras [258]. However, research and development are still underway to address these technical challenges and currently sub-millimetre acquisitions are not feasible. Lastly, while high-resolution ASL at ultra-high field is possible using the standard commercial head-coil (NOVA Medical, USA), $\mathrm{B}_{1}{ }^{+}$ field inhomogeneity is a major hurdle. While we were able to mitigate this to some extent using dielectric pads, advances in parallel transmission (pTx) technology and use of dedicated labelling-only RF-coils [259-262] can potentially further optimise high-resolution ASL fMRI at ultra-high field. 




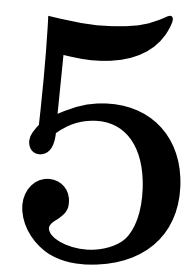

General Discussion 

Over the past decade, $7 \mathrm{~T}$ has grown from being a research-only platform that provides structural and functional images of the human body with an unparalleled amount of detail and contrast, to a clinical platform that provides improvements made to patient diagnosis and intervention with reduced scan durations. Recently, the improvements have allowed Siemens to receive CE and FDA certifications for their 7 T Terra system for clinical use. Functional MRI researchers, typically neuroscientists with a cursory education in MR-physics, are increasingly switching to $7 \mathrm{~T}$ given its significant advantages over conventional field strengths $(\leq 3 \mathrm{~T})$. Currently, the range of neuroscientific questions that can be addressed is becoming larger ( $\approx$ laminar/columnar circuitry), and the scale of cortical organisation and function becoming smaller $(\approx$ sub-millimetre, sub-second regimes $)$. While it is still possible for plug-and-play fMRI with standard coils and resolutions at $7 \mathrm{~T}$, similar to that at $3 \mathrm{~T}$, high-resolution fMRI comes with a lot of challenges that can be addressed to a large extent, by making informed choices regarding hardware, sequences, acquisition types and analysis strategies. Over the course of the studies reported in this thesis, we investigated different high-resolution anatomical and functional acquisition strategies, alternative contrast mechanisms and analysis approaches for laminar fMRI applications at ultra-high field ( $\geq 7 \mathrm{~T}$ ). In this chapter, we discuss the high-resolution fMRI acquisition, processing and analysis strategies that were adapted and developed over the course of this thesis and conclude with a summary.

\subsection{Data acquisition and processing}

A researcher having a well-controlled experimental design investigating laminar circuitry at $7 \mathrm{~T}$ using fMRI is faced with an overabundance of options when it comes to acquiring data. Decisions taken for the data acquisition are driven pri- 
marily by previous work reported in literature (most often at $3 \mathrm{~T}$ or lower) and legacy data analysis approaches which should "just work". The first step in this process and an often under-appreciated aspect of an fMRI study is choosing the right hardware. Almost every high-field site has specialised multi-receive array coils focussed on specific brain regions such as visual, auditory cortices etc. For example, if the research question pertains to the early visual cortex, the default is to choose the whole-brain coil (despite the less densely packed coil loops near the occipital lobe), even though the high-resolution functional data being acquired is of partial brain coverage i.e. limited to the early visual cortex. This choice is driven by convenience of conventional data processing tools which were for wholebrain analysis by design. However, it would be significantly advantageous, at this juncture, to consider the availability of specialised region-of-interest coils (such as, [197] used in Chapter 3) as they have densely packed coil arrays around the region-of-interest that enable achieving higher spatial resolution, acceleration factors and (t)SNR than the default whole-brain coils [263]. The visual coil, for instance, also has the additional advantage of having a greater field-of-view for stimulus presentation than its whole-brain counterpart. This comes at the cost of using new and unconventional data processing strategies which are often not well documented and have several additional compiler and library dependencies. Nevertheless, the advantages do outweigh the disadvantages of using specialised hardware and processing the data using new, command-line driven, unconventional tools are quite manageable. The next step is choosing the appropriate MRsequences for data acquisition. 


\subsubsection{Anatomical}

Choice of anatomical imaging sequence typically providing a $\mathrm{T}_{1}$ and/or $\mathrm{T}_{1}$-weighting for improved GM-WM delineations (e.g. MPRAGE [116], MP2RAGE [117], MEMPRAGE [264], MI-EPI [131]) per se may not very critical but depending on the sequence, it can require additional post-processing in order to be useful. Please note, several laminar fMRI studies acquire anatomical data at $3 \mathrm{~T}$ (see 1.1), this is but an example of the aforementioned legacy acquisition schemes (circa 2010). This is no longer a requirement as improved bias-correction algorithms such as N4 [265] for traditional MPRAGE (circumventing the need to acquire additional proton-density weighted images) or the (nearly) bias-free MP2RAGE are preferable. Since the challenges of signal inhomogeneities at ultra-high field can now be dealt with in post-processing, acquiring anatomical data at a lower field strength (in many cases, a luxury) need not be considered. An additional reason to avoid across field strength data acquisition, if avoidable, is that it comes with much more challenges such as requiring gradient non-linearity corrections and across-session co-registrations.

Despite its advantages, one of the reasons why MP2RAGE utilisation is often met with hesitance is that pre-processing algorithms developed for MPRAGE-like acquisitions do not work out of the box. For instance, the MP2RAGE T $\mathrm{T}_{1}-\mathrm{w}$ as it comes out of the scanner has a very noisy non-brain background (Fig. 5.1, left) and skull-stripping with conventional tools such as bet fail. ANTs and SPM offer strategies which are slightly convoluted workarounds. However, the author of the MP2RAGE paper has a simple script available on his Github page ${ }^{1}$, that gets rid of this by including a regularisation term to the image reconstruction and renders it very similar to an MPRAGE image(Fig. 5.1, right), and all the conventional tools

\footnotetext{
$1_{\text {https://github.com/JosePMarques/MP2RAGE-related-scripts }}$
} 

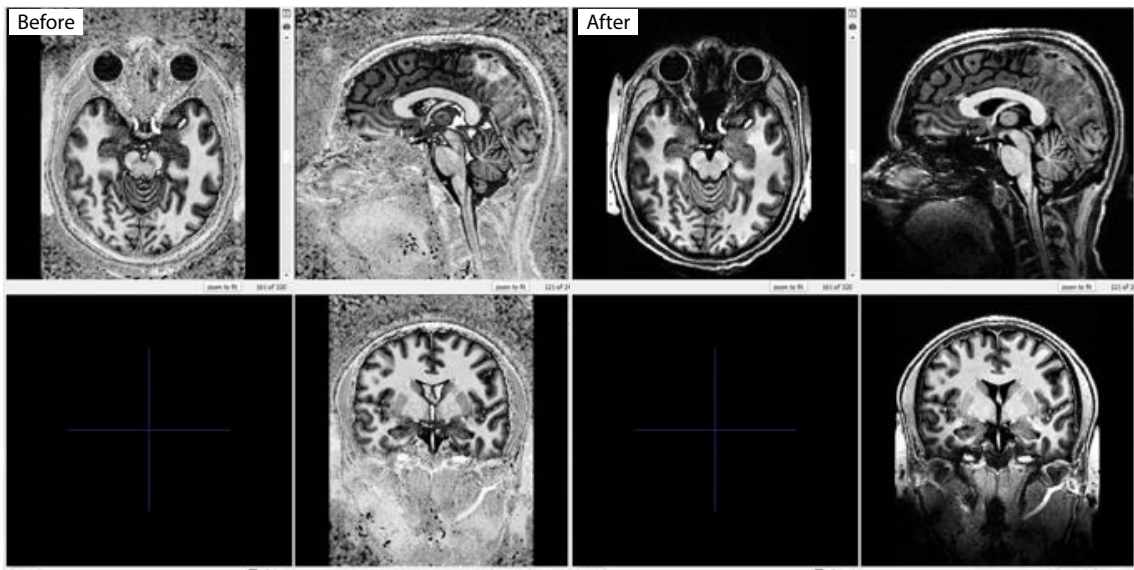

Figure 5.1: The background noise issue with MP2RAGE $T_{1}-\mathbf{w}$ image as obtained by default (left) and the regularised reconstruction using code by José Marques. (https://github.com/JosePMarques/ MP2RAGE-related-scripts/blob/master/DemoRemoveBackgroundNoise.m)

just work. Alternatively, a simpler processing step of multiplying the MP2RAGE $\mathrm{T}_{1}$-w by the second inversion image $(\approx$ proton density like contrast) also yields a similar result albeit requiring further intensity inhomogeneity correction. Another advantageous aspect of the MP2RAGE in this regard would be that the first inversion image is specifically optimised to have increased GM-WM contrast and the quantitative $\mathrm{T}_{1}$ map has increased CSF-GM contrast. Since all these images are in the same space, the two boundaries for laminar analysis can be extracted from these separate contrasts.

In the studies reported in this thesis, we decided to opt for the MP2RAGE sequence for a couple of reasons. First, the additional quantitative $\mathrm{T}_{1}$ map computed in the MP2RAGE was important for the $\mathrm{T}_{1}$ comparisons made in Chapter $\mathbf{2}$ and errors in $\mathrm{T}_{1}$ estimates due to $\mathrm{B}_{1}{ }^{+}$could be corrected post-hoc using an Sa2RAGE sequence. We show that these corrected- $\mathrm{T}_{1}$ estimates are in the same range as 
those estimated from a multiple inversion-recovery EPI (MI-EPI) sequence. Second, due to our use of a specialised visual cortex coil in Chapter 3 the Sa2RAGEcorrection proved critical to correct the strong $B_{1}$ in the occipital pole to aid better segmentation. In Chapter 4, having the additional contrasts such as the quantitative $\mathrm{T}_{1}$ image also allowed us to adapt the Freesurfer algorithm for pial surface optimisation designed to use $\mathrm{T}_{2}-\mathrm{w}$ images (e.g. SPACE). Although we have not tried using a multi-echo MPRAGE (MEMPRAGE [264]), it would be very interesting to see how the different contrasts can be useful, especially in conjunction with probabilistic frameworks (such as SPM's Unified Segmentation [145]) which can use the information content from multi-contrast data to aid better image segmentation.

\subsubsection{Functional}

The choices for high-resolution functional acquisition using $\mathrm{T}_{2}{ }^{*}$-weighting are FLASH [198], 2D-EPI [14] and 3D-EPI [141]. While FLASH has the advantage of having little-to-no geometric distortion due to $\mathrm{B}_{0}$ non-uniformity, it has poor temporal resolution [67] (e.g. one slice $\approx 1.76 \mathrm{~s}$ in Chapter 3). Therefore, for Chapters 2 and 4, it was clear we would use an EPI sequence, the decision was between 2D- or 3D-EPI for a nominal voxel resolution of $0.7 \mathrm{~mm}$ isotropic. After having carried out pilot experiments, we concluded that in thermal-noise dominated sub-millimetre voxel regimes, the 3D-EPI provided better tSNR and directly translated into more robust functional activation maps. This was later confirmed more systematically by Huber and colleagues [83] (Fig. 5.2) for both the BOLD and CBV-weighted signals using the SS-SI-VASO sequence. Therefore, for the EPI-based datasets in this thesis, we opted for the 3D-EPI acquisition. Additionally, Lutti and colleagues [266] have demonstrated that even for physiological- 


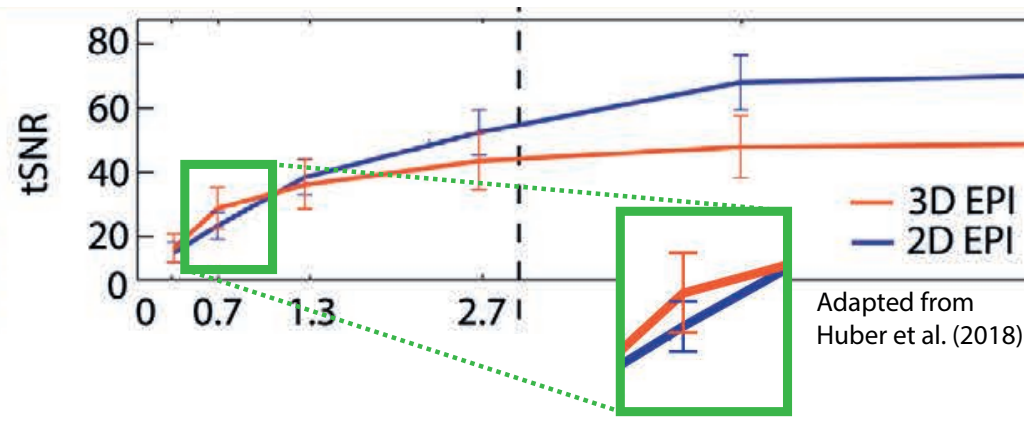

Figure 5.2: Plot of tSNR change with respect to voxel size using 2D- and 3D-EPI readouts (adapted from [83]). The zoomed in box highlights the sub-millimetre regime typically used for laminar fMRI.

noise dominated supra-millimetre regimes, correcting for physiological noise results in $\geq 25 \%$ increase in tSNR and better sensitivity than 2D-EPI. Our experience does not make the 3D-EPI prescriptive in any sense for all fMRI studies and we just note the importance of pilot experiments before choosing the optimal functional sequence for a study. Dealing with partial-coverage functional data in high-resolution fMRI is especially difficult with conventional data analysis strategies. Existing tools do not manage to deal with these datasets despite there being options to optimise these algorithms for such datasets. For example, something as simple as brain extraction (skull stripping) in FSL's bet has a special flag “-Z” when there are few slices in the z-direction. However, despite optimising parameters, it produces erroneous results (Fig. 5.3, left). Brain masking is quite crucial in highresolution studies because we want to limit the motion-correction/co-registration cost-function minimisations to the brain (even better if using a region-of-interest mask [82]). Here, ITK-SNAP [147] can be particularly useful. It's a highly versatile tool with an excellent set of semi-automated visually-guided segmentation algorithms, making it extremely intuitive to use. The clustering function in partic- 

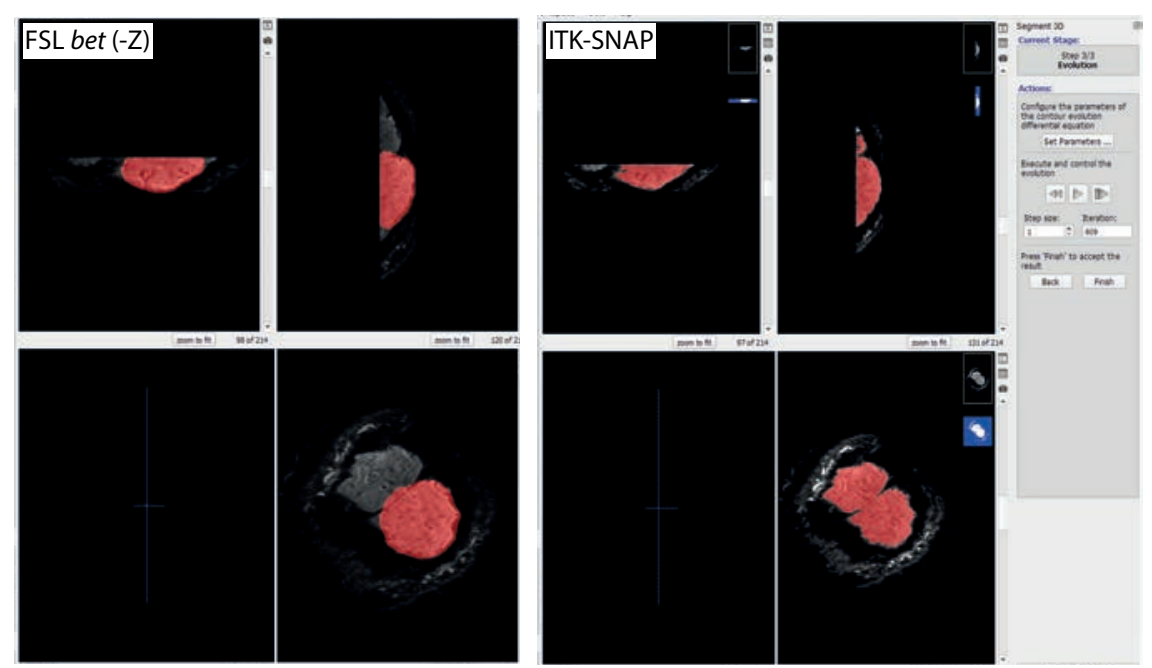

Figure 5.3: Examples of automatic skull-stripping/brain-extraction using FSL bet of partial coverage data and the same using ITK-SNAP.

ular can generate a highly accurate brain mask for partial-coverage data (Fig. 5.3, right) in $\leq 30 \mathrm{~s}$. ITK-SNAP was essential to create the initial alignment matrices for accurate co-registrations using the ANTs framework [184, 207] as in Chapters 4 and 5.

The fact that the anatomical and functional datasets are differently-distorted presents unique challenges. We address this topic exhaustively in Chapter 2. Furthermore, we show that it is feasible to use the distortion-matched MI-EPI quantitative $\mathrm{T}_{1}$ mapping approach with a specialised visual cortex coil in Chapter 3. This approach has picked up a lot of traction over the years since its publication and distortion-matched acquisitions have been made available with a 3D-EPI implementation called T123DEPI [267] which utilises an MP2RAGE-like reconstruction scheme. 

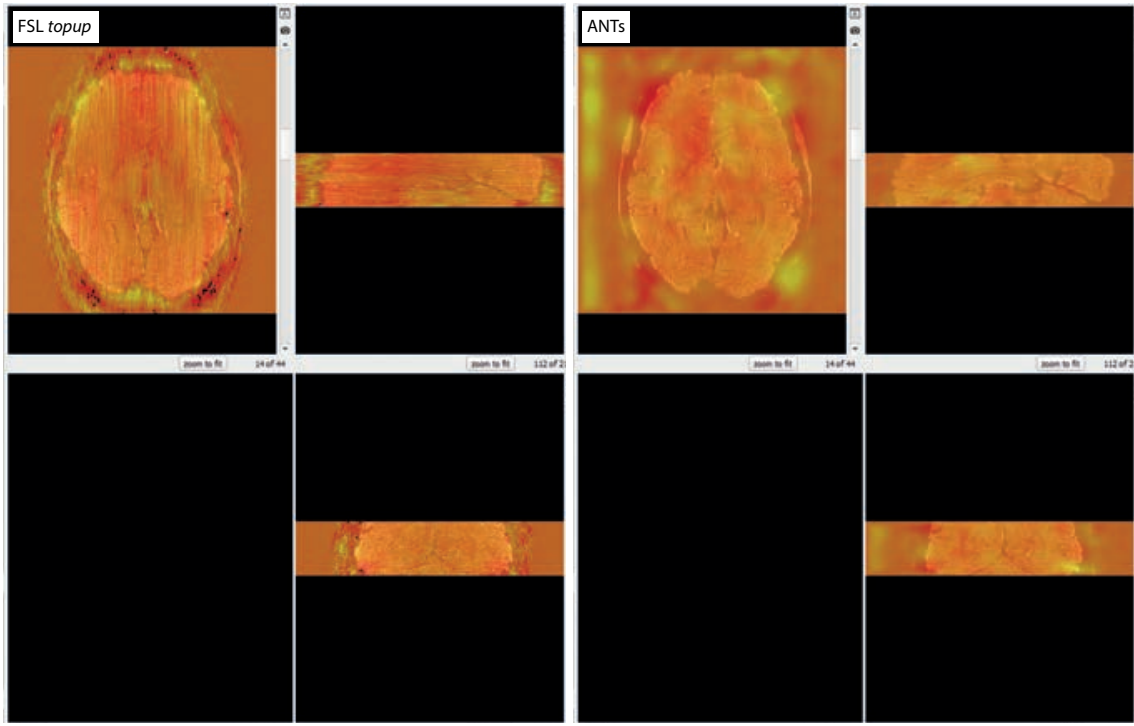

Figure 5.4: Voxel displacement maps from obtained from FSL topup and that from ANTs using a constrained SyN deformation.

In the cases where acquiring distortion-matched anatomical datasets was not possible (for e.g. due to time limitations) as in Chapter 4, we show that we can still minimally process the functional data (as espoused in Chapter 2) by adapting the tools available in the ANTs framework to apply motion-correction, distortioncorrection and co-registration to anatomical image in a single resampling step. The voxel-displacement maps by FSL topup (Fig. 5.4, left) are very jagged and appear to be slightly lower resolution than those estimated using ANTs (Fig. 5.4, right). This is despite the fact that the FSL topup configuration file (specifically warpres) was modified to account for the high-resolution data. Furthermore, ANTs can assess non-linear deformations along all axes, therefore, care was taken so as to ensure that the deformation step were restricted to the phase-encode axis only. The higher resolution warps estimated by ANTs, therefore, result in relatively less 
blurring in the images than with topup, therefore, likely preserving some of the acquired data's fidelity.

\subsection{Laminar analysis}

After having performed optimal processing of the anatomical and functional data, the next step is sampling the fMRI signal/statistical values across the cortical depths. An arbitrary number of cortical depths can be defined between the CSFGM and GM-WM bounds using either an distance-preserving (equi-distant) or volume-preserving (equi-volume) models (Fig. 5.5a, upper-panel). Following this, the fMRI signal can be resampled at these depths in order to achieve a finer separation of the laminar signals than the relatively coarser resolution of the data. Waehnert and colleagues [70] showed that the distance-preserving layering model is sub-optimal in regions of with high-curvature, whereas a volume-preserving layering model better follows the cyto-architecture. However, in our experience (data not shown, Chapter 2 ) at voxel resolutions $\approx 0.7-0.8 \mathrm{~mm}$ isotropic, and averaging over a region-of-interest did not reveal a significant difference between the two models for laminar sampling [75, 76]. Additionally, the equi-volume approach was infrequently used as only CBS-Tools [202] offered the algorithm bundled within a software package called MIPAV. Recently, due to its open-source nature, there are now community-driven scripts to use the equi-volume model with conventional tools such as Freesurfer [252] and the whole CBS-Tools algorithms have been repackaged in python as nighres. Once the layering is carried out, the depthdependent analysis is then done by resampling the fMRI signal onto dense meshes [66], binning voxels in an upsampled resolution [98] or using regular Cartesian grids [99]. Data resampling in this manner can result in spatial blurring, signal leakage across layers and reduced specificity [72]. 
a)
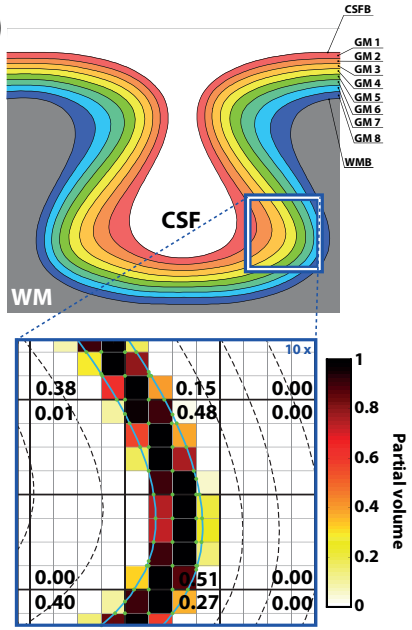

b)

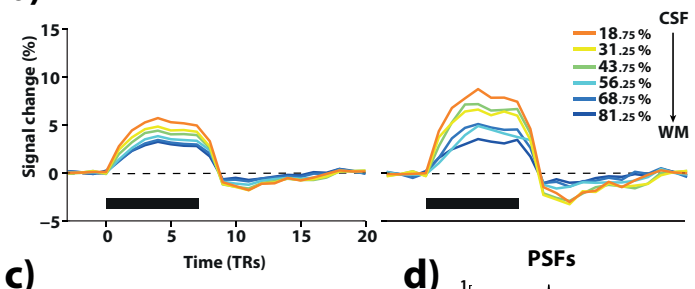

c)

d)

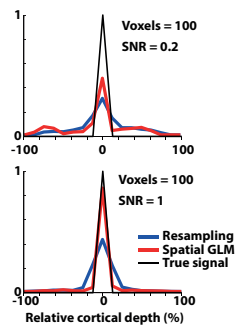

Figure 5.5: (a) top: equivolume layering on a curved model of cortex, bottom: a snapshot of the estimated the laminar-partial-volume-matrix. (b) Laminar time-courses by resampling (left) and spatial GLM (right). (c) Laminar profiles from the two sampling methods. (d) estimated PSFs for the sampling methods under realistic $(\mathrm{SNR}=0.2)$ and ideal $(\mathrm{SNR}=1)$ scenarios.

An alternative has been proposed to unmix the laminar signal using a spatial general linear model (spatial GLM) [72, 74, 182]. In this approach, a laminarpartial-volume matrix is estimated (Fig. 5.5a, bottom-panel) i.e., the space between two laminar surfaces defines the partial volume contribution of each layer to a voxel that spans several layers. Using data from Chapter 2, Havlicek and colleagues [72] have demonstrated the differences between the two approaches (Fig. 5.5b) on the sampled laminar time-courses and the corresponding laminar profiles (Fig. 5.5c) with the resampling approach providing smoother profiles whereas the spatial GLM has more structure to it while exhibiting a similar general trend. Figure 5.5d shows the estimated point-spread functions from simulated data by Havlicek and colleagues [72] for a realistic scenario ( $\mathrm{SNR}=0.2)$ and an idealistic scenario ( $\mathrm{SNR}=1)$, showing that in both cases, the spatial GLM approximates more 
closely to the ground-truth than the resampling approach. Nevertheless, this approach still requires systematic testing and validation.

Lastly, almost every laminar fMRI paper issues a disclaimer regarding the use of "lamina" to define computationally generated depths and not directly related to cytoarchitectonic layers. While there are plenty of arguments why the word "lamina" is not the sole custody of histologists (discussed at length in layerfmri.com), the

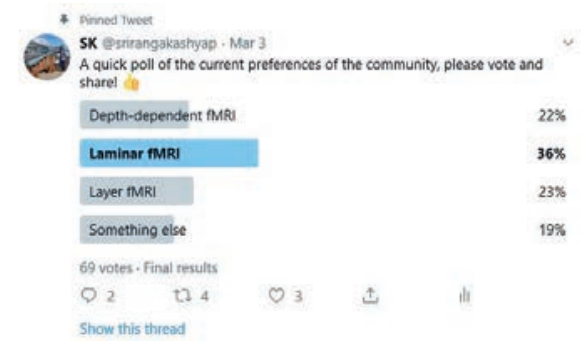

Figure 5.6: Results of a poll conducted on twitter (https://twitter.com/srirangakashyap/status/ $1102146422592491521)$ term's usage in fMRI is still not mainstream enough to merit its use without this disclaimer. A quick, informally conducted twitter poll (Fig. 5.6), revealed that laminar fMRI is preferred by a small majority followed closely by depth-dependent fMRI and layer fMRI despite there being no clear consensus. Nevertheless, this is the hallmark of an evolving field of research and such a high-degree of community engagement is definitely a reason for optimism.

\subsection{Vascular bias in GE-BOLD}

As introduced briefly in Chapter 1 and also addressed to some extent in Chapter 2, the GE-BOLD contrast used for the vast majority of laminar fMRI studies suffers from spatial confounds associated with intra-cortical ascending veins and draining pial veins. While the some of the pial veins can be spotted with high-resolution anatomical scans, the ascending veins are very difficult to resolve at the voxel resolutions possible thus far. These ascending veins collect 
the deoxy-Hb from the micro-vasculature at the site of neuronal activation and transports it to the pial vein at the surface. The distribution of these ascending veins and the accumulative effect of the deoxy-Hb introduces local biases in amplitude and localisation of the laminar BOLD signal in the cortex. There have been modelling efforts to address this intra-cortical drainage effect of the ascending vein [109, 110] and till date, only Marquardt and colleagues [195] have applied the steady-state Markuerkiaga model [110] to high-resolution fMRI data to deconvolve the ascending vein effect from their sampled laminar BOLD profiles. On the other hand, the Heinzle model [109] is a two-depth extension of the balloon model [268] within the dynamic causal modelling (DCM) framework [269] and has been recently improved upon by Havlicek and Uludağ [270] into a multi-compartment laminar BOLD model that is derived using mass conservation principles. Because the Havlicek-Uludağ laminar BOLD model is a fully dynamic model described by differential equations, it allows simulating steadystates and entire time-courses together with all its transients, such as response peak, early-overshoot, post-stimulus undershoot (PSU) or "the elusive" initial dip [271].

\subsection{Non-BOLD alternatives}

As discussed above, there have been significant strides made in addressing the limitations of the BOLD contrast using model-driven computational approaches and their application and validation will be critical as BOLD remains the contrastof-choice for many studies given its unrivalled sensitivity. However, there have also been significant developments made in making the non-BOLD functional contrasts such as the SS-SI-VASO (measuring CBV and BOLD) [180], 3D-EPI pulsed ASL (measuring CBF and BOLD) [238] (Chapter 4) feasible at ultra-high field 
and at sub-millimetre resolutions. This is particularly useful for laminar fMRI as both VASO and ASL offer better spatial specificity than BOLD but have limited sensitivity [80], with most applications arising from sensory cortices with stimuli that elicit very robust activation. Of the available alternatives, VASO has recently seen application to the dorso-lateral pre-frontal cortex (dlPFC) [272] apart from several laminar fMRI studies already carried out in the motor cortex [82, $166,180]$. On the other hand, CBF mapping (ASL) by virtue of the signal is inherently limited in SNR, even compared to total CBV mapping (VASO). This has seen limited application outside of clinical CBF mapping to high-resolution studies let alone fMRI. However, following significant sequence development and optimisation over the course of this thesis by Ivanov and colleagues [84, 88, 238, 273], we demonstrate, for the first time, the feasibility of using sub-millimetre spatial resolution 3D-EPI pulsed ASL for laminar fMRI in the visual cortex (Chapter 4). We hope to explore the full potential of the CBF contrast for laminar fMRI in future follow-up studies.

\subsection{Concluding remarks and outlook}

Since 2010, the field of laminar fMRI has grown at an extraordinary pace together with the increasing adoption of UHF MRI scanners. It is no surprise that one of the principal MRI journals, Neuroimage, came out with three Special Issues ${ }^{2}$ dedicated to developments encompassing optimised MR-sequences, post-processing algorithms, analysis tools and applications to cognitive neuroscience. Chapter 2 of this thesis emphasised minimal processing of functional data, demonstrated the feasibility of distortion-matched anatomical acquisitions to aid laminar analysis

\footnotetext{
${ }^{2}$ 1. Pushing the spatio-temporal limits of MRI and fMRI (Eds. Essa Yacoub, Larry Wald), 2. Neuroimaging with Ultra-high Field MRI: Present and Future (Eds. Jonathan Polimeni, Kâmil Uludağ), 3. in progress (Eds. Jonathan Polimeni and David Norris)
} 
in EPI space, raised the issue of co-registration inaccuracies even with distortioncorrection and showed the impact of conventional data processing strategies on the observed laminar profiles. The first laminar fMRI studies using EPI were carried out at $1 \mathrm{~mm}$ isotropic resolution [66], many following studies pushed this to $0.8 \mathrm{~mm}$ [99] and to $0.7 \mathrm{~mm}[89,98]$. While we do not know what the limits for spatial resolution are, what is clear is that there is no substitute for higher resolution. One way to push the boundary was inspired by line-scanning [79] animal fMRI by favouring ultra-high resolution along the dimension-of-interest (layers or columns) as we demonstrate using anisotropic voxels in Chapter 3. Future applications of this technique should incorporate some form of prospective motion correction either using optical-tracking, field-monitoring or even passively using custom designed head-cases ${ }^{3}$. Some preliminary results demonstrating the feasibility of a multi-echo version of the anisotropic FLASH fMRI in the motor cortex at 9.4 T was presented recently [274]. At ISMRM 2019, research groups in Boston [275] and Utrecht [276] have taken our approach further by actually implementing animal line-scanning like schemes for diffusion and fMRI respectively. This line of research is slowly, but surely gaining traction with many of the technological and analysis kinks to be addressed in the upcoming years. Finally, a take-away from a review of the studies in the field so far is that the functional neuroimaging community is now spoilt for choice when it comes to carrying out a laminar fMRI study. An example is that only a few years ago, the contrast of choice for high-resolution laminar fMRI in humans was BOLD (GE, SE, GRASE), however, CBV-weighted SS-SI-VASO has now enabled probing the laminar circuitry beyond sensory areas [82] such as the dorso-lateral pre-frontal cortex (dlPFC) [272]. In Chapter 4, we present CBF-weighted 3D-EPI ASL and demonstrate

${ }^{3}$ https://caseforge.co/ 
functional CBF mapping at sub-millimetre isotropic resolution for the first time in humans [207]. By showcasing the feasibility for laminar fMRI using CBF, we add yet another to the conundrum of choices for functional neuroimaging. Going forward, more studies using non-BOLD techniques addressing analysis and interpretability of the measured signals will hopefully see increased adoption to cognitive neuroscience applications taking full advantage of their quantitative nature and relatively better spatial specificity to neuronal activation compared to BOLD. Nevertheless, due to its popularity and unrivalled sensitivity, the BOLD contrast will stand the test of time. While major strides have been made in understanding the laminar specificity of the BOLD signal by modelling studies under the assumptions of steady-state [110] and more recently, from a fully dynamical [270] perspective within the framework of physiologically-informed dynamic causal modelling (P-DCM) [277], model-based deconvolution of laminar BOLD responses have only just started seeing application [195, 207] and additional validation studies will propel these model-driven techniques into mainstream adoption in laminar fMRI. 



\section{Bibliography}

[1] A. B. Nobel. Alfred Nobel's will. Nobel Media AB 2019. 1895. URL: https : / www. nobelprize. org/alfred-nobel/full-text-of-alfred-nobels-will-2/.

[2] I. Estermann and O. Stern. "Beugung von Molekularstrahlen”. In: Zeitschrift für Physik 61.1-2 (Jan. 1930), pp. 95-125.

[3] I. Estermann, R. Frisch, and O. Stern. "Magnetic Moment of the Proton". In: Nature 132.3326 (July 1933), pp. 169-170.

[4] R. Frisch and O. Stern. "Über die magnetische Ablenkung von Wasserstoffmolekülen und das magnetische Moment des Protons. I”. In: Zeitschrift für Physik 85.1-2 (Jan. 1933), pp. 4-16.

[5] I. I. Rabi, J. M. B. Kellogg, and J. R. Zacharias. "The Magnetic Moment of the Proton". In: Physical Review 46 (3 Aug. 1934), pp. 157-163.

[6] I. I. Rabi, J. R. Zacharias, S. Millman, and P. Kusch. "A New Method of Measuring Nuclear Magnetic Moment”. In: Physical Review 53 (4 Feb. 1938), pp. 318-318.

[7] I. I. Rabi, S. Millman, P. Kusch, and J. R. Zacharias. "The Molecular Beam Resonance Method for Measuring Nuclear Magnetic Moments. The Magnetic Moments of ${ }_{3} \mathrm{Li}^{6},{ }_{3} \mathrm{Li}^{7}$ and ${ }_{9} \mathrm{~F}^{19}$ ”. In: Physical Review 55 (6 Mar. 1939), pp. 526-535.

[8] F. Bloch, W. W. Hansen, and Martin Packard. "Nuclear Induction". In: Physical Review 69 (3-4 Feb. 1946), pp. 127-127.

[9] E. M. Purcell, H. C. Torrey, and R. V. Pound. "Resonance Absorption by Nuclear Magnetic Moments in a Solid”. In: Physical Review 69 (1-2 Jan. 1946), pp. 37-38.

[10] R.W. Brown, E.M. Haacke, Y.C.N. Cheng, M.R. Thompson, and R. Venkatesan. Magnetic Resonance Imaging: Physical Principles and Sequence Design. Wiley, 2014.

[11] E. L. Hahn. "Spin Echoes". In: Physical Review 80 (4 1950), pp. 580-594.

[12] M. A. Bernstein, K. F. King, and X. J. Zhou. "Chapter 16 - Echo Train Pulse Sequences". In: Handbook of MRI Pulse Sequences. Ed. by M. A. Bernstein, K. F. King, and X. J. Zhou. Burlington: Academic Press, 2004, pp. 702-801.

[13] P. C. Lauterbur. "Image Formation by Induced Local Interactions: Examples Employing Nuclear Magnetic Resonance”. In: Nature 242.5394 (Mar. 1973), pp. 190-191. 
[14] P. Mansfield. "Multi-planar image formation using NMR spin echoes". In: Journal of Physics C: Solid State Physics 10.3 (Feb. 1977), pp. L55-L58.

[15] P. Mansfield and A. A. Maudsley. "Medical imaging by NMR." In: The British Journal of Radiology 50 (591 Mar. 1977), pp. 188-94.

[16] G. M. Pohost, G. A. Elgavish, and W. T. Evanochko. "Nuclear magnetic resonance imaging: with or without nuclear?" In: Journal of the American College of Cardiology 7 (3 Mar. 1986), pp. 709 10 .

[17] W. C. Röntgen. "Ueber eine neue Art von Strahlen”. In: Annalen der Physik 300.1 (1895), pp. 1217.

[18] G. N. Hounsfield. "Computerized transverse axial scanning (tomography). 1. Description of system.” In: The British journal of radiology 46 (552 Dec. 1973), pp. 1016-22.

[19] J. W. Belliveau, D. N. Kennedy, R. C. McKinstry, et al. "Functional mapping of the human visual cortex by magnetic resonance imaging”. In: Science 254.5032 (1991), pp. 716-719.

[20] M. M. Ter-Pogossian, M. E. Phelps, E. J. Hoffman, and N. A. Mullani. "A Positron-Emission Transaxial Tomograph for Nuclear Imaging (PETT)”. In: Radiology 114.1 (1975), pp. 89-98.

[21] D. E. Kuhl and R. Q. Edwards. "Image Separation Radioisotope Scanning”. In: Radiology 80.4 (1963), pp. 653-662.

[22] M. M. Ter-Pogossian, J. O. Eichling, D. O. Davis, M. J. Welch, and J. M. Metzger. "The determination of regional cerebral blood flow by means of water labeled with radioactive oxygen 15 ." In: Radiology 93 (1 July 1969), pp. 31-40.

[23] R. L. Grubb Jr, M. E. Raichle, J. O. Eichling, and M. M. Ter-Pogossian. "The effects of changes in $\mathrm{PaCO}_{2}$ on cerebral blood volume, blood flow, and vascular mean transit time." In: Stroke 5 (5 Sept. 1974), pp. 630-9.

[24] A. Villringer, B. R. Rosen, J. W. Belliveau, et al. "Dynamic imaging with lanthanide chelates in normal brain: contrast due to magnetic susceptibility effects." In: Magnetic resonance in medicine 6 (2 Feb. 1988), pp. 164-74.

[25] B. R. Rosen, J. W. Belliveau, and D. Chien. "Perfusion imaging by nuclear magnetic resonance." In: Magnetic resonance quarterly 5 (4 Oct. 1989), pp. 263-81.

[26] M. F. Perutz, H. Muirhead, J. M. Cox, and L. C. G. Goaman. "Three-dimensional Fourier Synthesis of Horse Oxyhaemoglobin at $2.8 \AA \AA$ Resolution: The Atomic Model”. In: Nature 219.5150 (July 1968), pp. 131-139. 
[27] F. L. Hünefeld. Der Chemismus in der thierischen Organisation : physiologisch-chemische Untersuchungen der materiellen Veränderungen oder des Bildungslebens im thierischen Organismus, insbesondere des Blutbildungsprocesses, der Natur der Blutkörperchen und ihrer Kernchen : ein Beitrag zur Physiologie und Heilmittellehre. German. F.A. Brockhaus Leipzig, 1840.

[28] S.-Y. Park, T. Yokoyama, N. Shibayama, Y. Shiro, and J. R. H. Tame. "1.25 Å resolution crystal structures of human haemoglobin in the oxy, deoxy and carbonmonoxy forms." eng. In: Journal of molecular biology 360 (3 July 2006), pp. 690-701.

[29] M. Faraday. "Experimental Researches in Electricity. Twentieth Series". In: Philosophical Transactions of the Royal Society of London 136 (1846), pp. 21-40.

[30] L. Pauling and C. D. Coryell. "The Magnetic Properties and Structure of the Hemochromogens and Related Substances." In: Proceedings of the National Academy of Sciences of the United States of America 22 (3 Mar. 1936), pp. 159-63.

[31] K. R. Thulborn, J. C. Waterton, P. M. Matthews, and G. K. Radda. "Oxygenation dependence of the transverse relaxation time of water protons in whole blood at high field." eng. In: Biochimica et biophysica acta 714 (2 Feb. 1982), pp. 265-70.

[32] D. G. Norris. "Pulse Sequences for fMRI". In: fMRI: From Nuclear Spins to Brain Functions. Ed. by Kâmil Uludağ, Kâmil Uğurbil, and Lawrence Berliner. Boston, MA: Springer US, 2015, pp. 131-162.

[33] S. Ogawa, T. M. Lee, A. S. Nayak, and P. Glynn. "Oxygenation-sensitive contrast in magnetic resonance image of rodent brain at high magnetic fields". In: Magnetic resonance in medicine 14.1 (1990), pp. 68-78.

[34] P. T. Fox and M. E. Raichle. "Focal physiological uncoupling of cerebral blood flow and oxidative metabolism during somatosensory stimulation in human subjects”. In: Proceedings of the National academy of Sciences of the United States of America 83.4 (1986), pp. 1140-4.

[35] S. Ogawa, T. M. Lee, A. R. Kay, and D. W. Tank. "Brain magnetic resonance imaging with contrast dependent on blood oxygenation". In: Proceedings of the National academy of Sciences of the United States of America 87.24 (1990), pp. 9868-72.

[36] P. A. Bandettini, E. C. Wong, R. S. Hinks, R. S. Tikofsky, and J. S. Hyde. "Time course EPI of human brain function during task activation”. In: Magnetic Resonance in Medicine 25.2 (1992), pp. 390-397.

[37] K. K. Kwong, J. W. Belliveau, D. A. Chesler, et al. "Dynamic magnetic resonance imaging of human brain activity during primary sensory stimulation”. In: Proceedings of the National Academy of Sciences 89.12 (June 1992), pp. 5675-9. 
[38] S. Ogawa, D. W. Tank, R. Menon, et al. "Intrinsic signal changes accompanying sensory stimulation: functional brain mapping with magnetic resonance imaging”. In: Proceedings of the National academy of Sciences of the United States of America 89.13 (1992), pp. 5951-5.

[39] K. Uludağ, K. Uğurbil, and L. Berliner. fMRI: From Nuclear Spins to Brain Functions. Biological Magnetic Resonance. Springer US, 2015.

[40] K. Uğurbil and S. Ogawa. "From BOLD Contrast to Imaging Human Brain Function". In: fMRI: From Nuclear Spins to Brain Functions. Ed. by Kâmil Uludağ, Kâmil Uğurbil, and Lawrence Berliner. Boston, MA: Springer US, 2015, pp. 3-9.

[41] P. A. Bandettini. "The Birth of Functional MRI at the Medical College of Wisconsin". In: $f M R I$ : From Nuclear Spins to Brain Functions. Ed. by Kâmil Uludağ, Kâmil Uğurbil, and Lawrence Berliner. Boston, MA: Springer US, 2015, pp. 11-18.

[42] K. K. Kwong. "The Road to fMRI Using Endogenous MR Blood Contrast". In: fMRI: From Nuclear Spins to Brain Functions. Ed. by Kâmil Uludağ, Kâmil Uğurbil, and Lawrence Berliner. Boston, MA: Springer US, 2015, pp. 19-23.

[43] K. Uludağ, B. Muller-Bierl, and K. Uğurbil. "An integrative model for neuronal activity-induced signal changes for gradient and spin echo functional imaging”. In: Neuroimage 48.1 (2009), pp. $150-65$.

[44] C. S. Roy and C. S. Sherrington. "On the Regulation of the Blood-supply of the Brain." eng. In: The Journal of physiology 11 (1-2 Jan. 1890), pp. 85-158.17.

[45] N. K. Logothetis, J. Pauls, M. Augath, T. Trinath, and A. Oeltermann. "Neurophysiological investigation of the basis of the fMRI signal”. In: Nature 412.6843 (2001), pp. 150-7.

[46] N. K. Logothetis. "MR imaging in the non-human primate: studies of function and of dynamic connectivity". In: Current Opinion in Neurobiology 13.5 (Oct. 2003), pp. 630-642.

[47] N. K. Logothetis. "The neural basis of the blood-oxygen-level-dependent functional magnetic resonance imaging signal”. In: Philosophical Transactions of the Royal Society of London. Series B, Biological Sciences 357.1424 (2002), pp. 1003-37.

[48] D. Attwell and C. Iadecola. "The neural basis of functional brain imaging signals". In: Trends in Neurosciences 25.12 (2002), pp. 621-5.

[49] K. Uludağ and P. Blinder. "Linking brain vascular physiology to hemodynamic response in ultrahigh field MRI”. In: Neuroimage 168 (2018), pp. 279-295.

[50] K. Uludağ and K. Uğurbil. "Physiology and Physics of the fMRI Signal”. In: fMRI: From Nuclear Spins to Brain Functions. Ed. by Kâmil Uludağ, Kâmil Uğurbil, and Lawrence Berliner. Boston, MA: Springer US, 2015, pp. 163-213. 
[51] K Uludağ and M Havlicek. "Hemodynamic modeling of laminar resolution fMRI". In: Paris, France, 2018.

[52] D. G. Norris. "Spin-echo fMRI: The poor relation?" In: Neuroimage 62.2 (2012), pp. 1109-15.

[53] R. Pohmann, O. Speck, and K. Scheffler. "Signal-to-noise ratio and MR tissue parameters in human brain imaging at 3, 7, and 9.4 tesla using current receive coil arrays". In: Magnetic Resonance in Medicine 75.2 (2016), pp. 801-809.

[54] K. Uğurbil. "Imaging at ultrahigh magnetic fields: History, challenges, and solutions." eng. In: Neuroimage 168 (Mar. 2018), pp. 7-32.

[55] B. A. Poser and K. Setsompop. "Pulse sequences and parallel imaging for high spatiotemporal resolution MRI at ultra-high field.” eng. In: Neuroimage 168 (Mar. 2018), pp. 101-118.

[56] E. Yacoub, T. Q. Duong, P. F. Van De Moortele, et al. "Spin-echo fMRI in humans using high spatial resolutions and high magnetic fields". In: Magnetic Resonance in Medicine 49.4 (2003), pp. 655-64.

[57] J. B. Goense and N. K. Logothetis. "Laminar specificity in monkey V1 using high-resolution SE-fMRI”. In: Magnetic Resonance Imaging 24.4 (2006), pp. 381-92.

[58] K. Brodmann. Vergleichende Lokalisationslehre der Grosshirnrinde in ihren Prinzipien dargestellt auf Grund des Zellenbaues. J.A. Barth, 1909.

[59] O. Vogt. Die myeloarchitektonische Felderung des menschlichen Stirnhirns. J.A. Barth, 1910.

[60] R. J. Douglas and K. A. Martin. "Neuronal circuits of the neocortex". In: Annual Review of Neuroscience 27 (2004), pp. 419-51.

[61] B. Weber, A. L. Keller, J. Reichold, and N. K. Logothetis. "The microvascular system of the striate and extrastriate visual cortex of the macaque”. In: Cerebral Cortex 18.10 (2008), pp. 2318-30.

[62] D. L. Adams, V. Piserchia, J. R. Economides, and J. C. Horton. "Vascular Supply of the Cerebral Cortex is Specialized for Cell Layers but Not Columns”. In: Cerebral Cortex 25.10 (Sept. 2014), pp. 3673-3681.

[63] D. Ress, G. H. Glover, J. Liu, and B. Wandell. "Laminar profiles of functional activity in the human brain”. In: Neuroimage 34.1 (2007), pp. 74-84.

[64] K. Cheng, R. A. Waggoner, and K. Tanaka. "Human Ocular Dominance Columns as Revealed by High-Field Functional Magnetic Resonance Imaging”. In: Neuron 32.2 (Oct. 2001), pp. 359-374.

[65] E. Yacoub, A. Shmuel, N. Logothetis, and K. Uğurbil. "Robust detection of ocular dominance columns in humans using Hahn Spin Echo BOLD functional MRI at 7 Tesla”. In: NeuroImage 37.4 (Oct. 2007), pp. 1161-1177. 
[66] J. R. Polimeni, B. Fischl, D. N. Greve, and L. L. Wald. "Laminar analysis of 7T BOLD using an imposed spatial activation pattern in human V1”. In: Neuroimage 52.4 (2010), pp. 1334-1346.

[67] P. J. Koopmans, M. Barth, S. Orzada, and D. G. Norris. "Multi-echo fMRI of the cortical laminae in humans at 7T". In: Neuroimage 56.3 (2011), pp. 1276-1285.

[68] P. J. Koopmans, M. Barth, and D. G. Norris. "Layer-specific BOLD activation in human V1". In: Human Brain Mapping 31.9 (2010), pp. 1297-304.

[69] S. E. Jones, B. R. Buchbinder, and I. Aharon. "Three-dimensional mapping of cortical thickness using Laplace's equation”. In: Hum Brain Mapp 11.1 (2000). Jones, S E Buchbinder, B R Aharon, I Journal Article United States Hum Brain Mapp. 2000 Sep;11(1):12-32., pp. 12-32.

[70] M. D. Waehnert, J. Dinse, M. Weiss, et al. "Anatomically motivated modeling of cortical laminae". In: Neuroimage 93 Pt 2 (2014), pp. 210-20.

[71] Y. Leprince, F. Poupon, T. Delzescaux, D. Hasboun, C. Poupon, and D. Rivière. "Combined LaplacianEquivolumic Model For Studying Cortical Lamination With Ultra High Field MRI (7T)”. In: 2015 IEEE 12th International Symposium on Biomedical Imaging (ISBI) (2015), pp. 580-583.

[72] M. Havlicek, S. Kashyap, D. Ivanov, and K. Uludağ. "Towards an optimal analysis of laminarresolved fMRI”. In: Proceedings of the OHBM. Geneva, Switzerland, 2016, pp. 7273-7273.

[73] P. Kok, L. J. Bains, T. van Mourik, D. G. Norris, and F. P. de Lange. "Selective Activation of the Deep Layers of the Human Primary Visual Cortex by Top-Down Feedback". In: Current Biology 26.3 (2016), pp. 371-6.

[74] T. van Mourik, J. P. J. M. van der Eerden, P.-L. Bazin, and D. G. Norris. "Laminar signal extraction over extended cortical areas by means of a spatial GLM”. In: bioRxiv (2018).

[75] J. R. Polimeni, V. Renvall, N. Zaretskaya, and B. Fischl. "Analysis strategies for high-resolution UHF-fMRI data”. In: Neuroimage 168 (Mar. 2018), pp. 296-320.

[76] V. G. Kemper, F. De Martino, T. C. Emmerling, E. Yacoub, and R. Goebel. "High resolution data analysis strategies for mesoscale human functional MRI at 7 and 9.4T". In: Neuroimage 164 (Jan. 2018), pp. 48-58.

[77] L. Muckli, F. De Martino, L. Vizioli, et al. "Contextual Feedback to Superficial Layers of V1". In: Current Biology 25.20 (2015), pp. 2690-5.

[78] P. Jezzard and S. Clare. "Sources of distortion in functional MRI data". In: Human Brain Mapping 8.2-3 (1999), pp. 80-5.

[79] X. Yu, C. Qian, D. Y. Chen, S. J. Dodd, and A. P. Koretsky. "Deciphering laminar-specific neural inputs with line-scanning fMRI”. In: Nature Methods 11.1 (2014), pp. 55-8. 
[80] L. Huber, K. Uludağ, and H. E. Moller. "Non-BOLD contrast for laminar fMRI in humans: CBF, CBV, and CMRO2." eng. In: Neuroimage (July 2017).

[81] H. Lu, X. Golay, J. J. Pekar, and P. C. Van Zijl. "Functional magnetic resonance imaging based on changes in vascular space occupancy”. In: Magnetic Resonance in Medicine 50.2 (2003), pp. 26374.

[82] L. Huber, D. A. Handwerker, D. C. Jangraw, et al. "High-Resolution CBV-fMRI Allows Mapping of Laminar Activity and Connectivity of Cortical Input and Output in Human M1.” eng. In: Neuron 96 (6 Dec. 2017), 1253-1263.e7.

[83] L. Huber, D. Ivanov, D. A. Handwerker, et al. "Techniques for blood volume fMRI with VASO: From low-resolution mapping towards sub-millimeter layer-dependent applications". In: Neuroimage 164 (2018), pp. 131-143.

[84] D. Ivanov, A. Gardumi, R. A. M. Haast, J. Pfeuffer, B. A. Poser, and K. Uludağ. "Comparison of $3 \mathrm{~T}$ and 7T ASL techniques for concurrent functional perfusion and BOLD studies." eng. In: Neuroimage 156 (Aug. 2017), pp. 363-376.

[85] S.-G. Kim. "Quantification of relative cerebral blood flow change by flow-sensitive alternating inversion recovery (FAIR) technique: Application to functional mapping”. In: Magnetic Resonance in Medicine 34.3 (1995), pp. 293-301.

[86] J. Goense, Y. Bohraus, and N. K. Logothetis. "fMRI at High Spatial Resolution: Implications for BOLD-Models”. In: Frontiers in Computational Neuroscience 10 (2016), p. 66.

[87] J. Pfeuffer, G. Adriany, A. Shmuel, et al. "Perfusion-based high-resolution functional imaging in the human brain at 7 Tesla”. In: Magnetic Resonance in Medicine 47.5 (Feb. 2002), pp. 903-911.

[88] D. Ivanov, B. A. Poser, L. Huber, J. Pfeuffer, and K. Uludağ. "Optimization of simultaneous multislice EPI for concurrent functional perfusion and BOLD signal measurements at 7T". In: Magnetic Resonance in Medicine (2016).

[89] S. Kashyap, D. Ivanov, M. Havlicek, B. A. Poser, and K. Uludağ. "Impact of acquisition and analysis strategies on cortical depth-dependent fMRI”. In: Neuroimage 168 (2018), pp. 332-344.

[90] C.F. von Economo and G.N. Koskinas. Die Cytoarchitektonik der Hirnrinde des erwachsenen Menschen. Die cytoarchitektonik der hirnrinde des erwachsenen menschen. J. Springer, 1925.

[91] J. Pfeuffer, P. F. van de Moortele, E. Yacoub, et al. "Zoomed functional imaging in the human brain at 7 Tesla with simultaneous high spatial and high temporal resolution”. In: Neuroimage 17.1 (2002), pp. 272-286. 
[92] E. Yacoub, N. Harel, and K. Uğurbil. "High-field fMRI unveils orientation columns in humans". In: Proceedings of the National academy of Sciences of the United States of America 105.30 (2008), pp. 10607-12.

[93] F. De Martino, M. Moerel, K. Uğurbil, R. Goebel, E. Yacoub, and E. Formisano. "Frequency preference and attention effects across cortical depths in the human primary auditory cortex". In: Proceedings of the National academy of Sciences of the United States of America 112.52 (2015), pp. 16036-41.

[94] F. De Martino, J. Zimmermann, L. Muckli, K. Uğurbil, E. Yacoub, and R. Goebel. "Cortical Depth Dependent Functional Responses in Humans at 7T: Improved Specificity with 3D GRASE”. In: PLOS ONE 8.3 (2013), pp. 30-32.

[95] A. Fracasso, P. R. Luijten, S. O. Dumoulin, and N. Petridou. "Laminar imaging of positive and negative BOLD in human visual cortex at 7T". In: Neuroimage (2018).

[96] L. Huber, D. Ivanov, M. Guidi, et al. "Functional cerebral blood volume mapping with simultaneous multi-slice acquisition”. In: Neuroimage 125 (2016), pp. 1159-1168.

[97] V. G. Kemper, F. De Martino, A. T. Vu, et al. "Sub-millimeter T2 weighted fMRI at 7T: comparison of 3D-GRASE and 2D SE-EPI". In: Frontiers in Neuroscience 9 (2015), p. 163.

[98] C. A. Olman, N. Harel, D. A. Feinberg, et al. "Layer-specific fMRI reflects different neuronal computations at different depths in human V1". In: PLOS ONE 7.3 (2012), e32536.

[99] J. Zimmermann, R. Goebel, F. De Martino, et al. "Mapping the organization of axis of motion selective features in human area MT using high-field fMRI". In: PLOS ONE 6.12 (2011), e28716.

[100] F. G. C. Hoogenraad, M. B. M. Hofman, P. J. W. Pouwels, J. R. Reichenbach, S. A. R. B. Rombouts, and E. M. Haacke. "Sub-millimeter fMRI at 1.5 Tesla: Correlation of high resolution with low resolution measurements". In: Jmri-Journal of Magnetic Resonance Imaging 9.3 (1999), pp. 475482 .

[101] N. K. Logothetis, H. Merkle, M. Augath, T. Trinath, and K. Uğurbil. "Ultra high-resolution fMRI in monkeys with implanted RF coils”. In: Neuron 35.2 (2002), pp. 227-42.

[102] J. B. Mandeville, J. J. Marota, B. E. Kosofsky, et al. "Dynamic functional imaging of relative cerebral blood volume during rat forepaw stimulation”. In: Magnetic Resonance in Medicine 39.4 (1998), pp. 615-24.

[103] J.T. Vaughan and J.R. Griffiths. RF Coils for MRI. 2012.

[104] M. A. Griswold, P. M. Jakob, R. M. Heidemann, et al. "Generalized autocalibrating partially parallel acquisitions (GRAPPA)”. In: Magnetic Resonance in Medicine 47.6 (2002), pp. 1202-10. 
[105] K. P. Pruessmann, M. Weiger, M. B. Scheidegger, and P. Boesiger. "SENSE: sensitivity encoding for fast MRI”. In: Magnetic Resonance in Medicine 42.5 (1999), pp. 952-962.

[106] K. Setsompop, D. A. Feinberg, and J. R. Polimeni. "Rapid brain MRI acquisition techniques at ultra-high fields". In: NMR in Biomedicine 29.9 (2016), pp. 1198-221.

[107] J. T. Vaughan, M. Garwood, C. M. Collins, et al. "7T vs. 4T: RF power, homogeneity, and signalto-noise comparison in head images”. In: Magnetic Resonance in Medicine 46.1 (2001), pp. 2430 .

[108] K. Uğurbil, L. Toth, and D-S Kim. "How accurate is magnetic resonance imaging of brain function?" In: Trends in Neurosciences 26.2 (2003), pp. 108-114.

[109] J. Heinzle, P. J. Koopmans, H. E. M. den Ouden, S. Raman, and K. E. Stephan. "A hemodynamic model for layered BOLD signals”. In: Neuroimage 125 (2016), pp. 556-570.

[110] I. Markuerkiaga, M. Barth, and D. G. Norris. "A cortical vascular model for examining the specificity of the laminar BOLD signal”. In: Neuroimage 132 (2016), pp. 491-498.

[111] N. Harel, J. Lin, S. Moeller, K. Uğurbil, and E. Yacoub. “Combined imaging-histological study of cortical laminar specificity of fMRI signals”. In: Neuroimage 29.3 (2006), pp. 879-87.

[112] D. S. Kim, T. Q. Duong, and S.-G. Kim. "High-resolution mapping of iso-orientation columns by fMRI". In: Nature Neuroscience 3.2 (2000), pp. 164-9.

[113] N. K. Logothetis. "What we can do and what we cannot do with fMRI". In: Nature 453.7197 (2008), pp. 869-878.

[114] C. Mathiesen, K. Caesar, N. Akgoren, and M. Lauritzen. "Modification of activity-dependent increases of cerebral blood flow by excitatory synaptic activity and spikes in rat cerebellar cortex". In: J Physiol 512 ( Pt 2).2 (1998), pp. 555-66.

[115] K. Uğurbil, G. Adriany, P. Andersen, et al. "Ultrahigh field magnetic resonance imaging and spectroscopy”. In: Magnetic Resonance Imaging 21.10 (2003), pp. 1263-81.

[116] J. P. Mugler III and J. R. Brookeman. "Three-dimensional magnetization-prepared rapid gradientecho imaging (3D MP RAGE)”. In: Magnetic Resonance in Medicine 15.1 (1990), pp. 152-7.

[117] J. P. Marques, T. Kober, G. Krueger, W. van der Zwaag, P. F. Van de Moortele, and R. Gruetter. "MP2RAGE, a self bias-field corrected sequence for improved segmentation and T1-mapping at high field”. In: Neuroimage 49.2 (2010), pp. 1271-1281.

[118] S. Nasr, J. R. Polimeni, and R. B. H. Tootell. "Interdigitated Color- and Disparity-Selective Columns within Human Visual Cortical Areas V2 and V3”. In: Journal of Neuroscience 36.6 (2016), pp. 1841-1857. 
[119] P. Jezzard. "Correction of geometric distortion in fMRI data”. In: Neuroimage 62.2 (2012), pp. 64851.

[120] P. Jezzard and R. S. Balaban. "Correction for geometric distortion in echo planar images from B0 field variations". In: Magnetic Resonance in Medicine 34.1 (1995), pp. 65-73.

[121] J. L. R. Andersson, S. Skare, and J. Ashburner. "How to correct susceptibility distortions in spinecho echo-planar images: application to diffusion tensor imaging”. In: Neuroimage 20.2 (2003), pp. 870-888.

[122] L. Fritz, J. Mulders, H. Breman, et al. "Comparison of EPI distortion correction methods at 3T and 7T". In: 2014.

[123] C. Hutton, A. Bork, O. Josephs, R. Deichmann, J. Ashburner, and R. Turner. "Image distortion correction in fMRI: A quantitative evaluation”. In: Neuroimage 16.1 (2002), pp. 217-40.

[124] P. Munger, G. R. Crelier, T. M. Peters, and G. B. Pike. "An inverse problem approach to the correction of distortion in EPI images”. In: IEEE Trans Med Imaging 19.7 (2000), pp. 681-9.

[125] S. Clare and P. Jezzard. "Rapid T(1) mapping using multislice echo planar imaging”. In: Magnetic Resonance in Medicine 45.4 (2001), pp. 630-4.

[126] P. Gowland and P. Mansfield. “Accurate measurement of T1 in vivo in less than 3 seconds using echo-planar imaging”. In: Magnetic Resonance in Medicine 30.3 (1993), pp. 351-354.

[127] P. Mansfield, D. N. Guilfoyle, R. J. Ordidge, and R. E. Coupland. "Measurement of T1 by echoplanar imaging and the construction of computer-generated images”. In: Physics in Medicine and Biology 31.2 (1986), pp. 113-24.

[128] R. J. Ordidge, P. Gibbs, B. Chapman, M. K. Stehling, and P. Mansfield. "High-Speed Multislice T1 Mapping Using Inversion-Recovery Echo-Planar Imaging”. In: Magnetic Resonance in Medicine 16.2 (1990), pp. 238-245.

[129] M. K. Stehling, R. J. Ordidge, R. Coxon, and P. Mansfield. "Inversion-Recovery Echo-Planar Imaging (Ir-Epi) at 0.5-T”. In: Magnetic Resonance in Medicine 13.3 (1990), pp. 514-517.

[130] M. K. Stehling, R. Turner, and P. Mansfield. "Echo-planar imaging: magnetic resonance imaging in a fraction of a second". In: Science 254.5028 (1991), pp. 43-50.

[131] V. Renvall, T. Witzel, L. L. Wald, and J. R. Polimeni. "Automatic cortical surface reconstruction of high-resolution T1 echo planar imaging data”. In: Neuroimage 134 (2016), pp. 338-354.

[132] F. Zhao, T. Jin, P. Wang, and S. G. Kim. "Improved spatial localization of post-stimulus BOLD undershoot relative to positive BOLD”. In: Neuroimage 34.3 (2007), pp. 1084-92. 
[133] F. Zhao, P. Wang, K. Hendrich, K. Uğurbil, and S. G. Kim. "Cortical layer-dependent BOLD and CBV responses measured by spin-echo and gradient-echo fMRI: insights into hemodynamic regulation”. In: Neuroimage 30.4 (2006), pp. 1149-60.

[134] S. Sadaghiani, K. Uğurbil, and K. Uludağ. "Neural activity-induced modulation of BOLD poststimulus undershoot independent of the positive signal”. In: Magnetic Resonance Imaging 27.8 (2009), pp. 1030-8.

[135] K. J. Mullinger, S. D. Mayhew, A. P. Bagshaw, R. Bowtell, and S. T. Francis. "Poststimulus undershoots in cerebral blood flow and BOLD fMRI responses are modulated by poststimulus neuronal activity". In: Proceedings of the National academy of Sciences of the United States of America 110.33 (2013), pp. 13636-41.

[136] J. W. Peirce. "PsychoPy-Psychophysics software in Python". In: Journal of Neuroscience Methods 162.1-2 (2007), pp. 8-13.

[137] F. Eggenschwiler, T. Kober, A. W. Magill, R. Gruetter, and J. P. Marques. "SA2RAGE: a new sequence for fast B1+ -mapping”. In: Magnetic Resonance in Medicine 67.6 (2012), pp. 1609-19.

[138] J. P. Marques and R. Gruetter. "New developments and applications of the MP2RAGE sequencefocusing the contrast and high spatial resolution R1 mapping”. In: PLOS ONE 8.7 (2013), e69294.

[139] A. C. Hurley, A. Al-Radaideh, L. Bai, et al. "Tailored RF Pulse for Magnetization Inversion at Ultrahigh Field”. In: Magnetic Resonance in Medicine 63.1 (2010), pp. 51-58.

[140] J. R. Polimeni, H. Bhat, T. Witzel, et al. "Reducing sensitivity losses due to respiration and motion in accelerated echo planar imaging by reordering the autocalibration data acquisition”. In: Magnetic Resonance in Medicine 75.2 (2016), pp. 665-79.

[141] B. A. Poser, P. J. Koopmans, T. Witzel, L. L. Wald, and M. Barth. "Three dimensional echo-planar imaging at 7 Tesla”. In: Neuroimage 51.1 (2010), pp. 261-266.

[142] R. A. M. Haast, D. Ivanov, E. Formisano, and K. Uludağ. "Reproducibility and Reliability of Quantitative and Weighted T1 and T2(*) Mapping for Myelin-Based Cortical Parcellation at 7 Tesla”. In: Frontiers in Neuroanatomy 10.112 (2016), p. 112.

[143] S. M. Smith, M. Jenkinson, M. W. Woolrich, et al. "Advances in functional and structural MR image analysis and implementation as FSL”. In: Neuroimage 23 Suppl 1 (2004), S208-19.

[144] N. Stikov, M. Boudreau, I. R. Levesque, C. L. Tardif, J. K. Barral, and G. B. Pike. "On the accuracy of T1 mapping: searching for common ground". In: Magnetic Resonance in Medicine 73.2 (2015), pp. 514-22.

[145] J. Ashburner and K. J. Friston. "Unified segmentation”. In: Neuroimage 26.3 (2005), pp. 839-51. 
[146] J. Ashburner. "A fast diffeomorphic image registration algorithm". In: Neuroimage 38.1 (2007), pp. 95-113.

[147] P. A. Yushkevich, J. Piven, H. C. Hazlett, et al. "User-guided 3D active contour segmentation of anatomical structures: significantly improved efficiency and reliability”. In: Neuroimage 31.3 (2006), pp. 1116-28.

[148] M. W. Woolrich, B. D. Ripley, M. Brady, and S. M. Smith. "Temporal autocorrelation in univariate linear modeling of FMRI data”. In: Neuroimage 14.6 (2001), pp. 1370-86.

[149] C. F. Beckmann, M. Jenkinson, and S. M. Smith. "General multilevel linear modeling for group analysis in FMRI”. In: Neuroimage 20.2 (2003), pp. 1052-63.

[150] M. W. Woolrich, T. E. Behrens, C. F. Beckmann, M. Jenkinson, and S. M. Smith. "Multilevel linear modelling for FMRI group analysis using Bayesian inference”. In: Neuroimage 21.4 (2004), pp. $1732-47$.

[151] J. L. Andersson, C. Hutton, J. Ashburner, R. Turner, and K. Friston. "Modeling geometric deformations in EPI time series". In: Neuroimage 13.5 (2001), pp. 903-19.

[152] W.D. Penny, K.J. Friston, J.T. Ashburner, S.J. Kiebel, and T.E. Nichols. Statistical Parametric Mapping: The Analysis of Functional Brain Images. Elsevier Science, 2011.

[153] J. C. Siero, J. Hendrikse, H. Hoogduin, N. Petridou, P. Luijten, and M. J. Donahue. "Cortical depth dependence of the BOLD initial dip and poststimulus undershoot in human visual cortex at 7 Tesla”. In: Magnetic Resonance in Medicine 73.6 (2015), pp. 2283-95.

[154] J. S. Gati, R. S. Menon, K. Uğurbil, and B. K. Rutt. "Experimental determination of the BOLD field strength dependence in vessels and tissue”. In: Magnetic Resonance in Medicine 38.2 (1997), pp. 296-302.

[155] E. M. Haacke, A. Hopkins, S. Lai, et al. "2D and 3D high resolution gradient echo functional imaging of the brain: venous contributions to signal in motor cortex studies”. In: NMR in Biomedicine 7.1-2 (Mar. 1994), pp. 54-62.

[156] R. S. Menon. "Postacquisition suppression of large-vessel BOLD signals in high-resolution fMRI". In: Magnetic Resonance in Medicine 47.1 (Dec. 2001), pp. 1-9.

[157] J. R. Polimeni, T. Witzel, B. Fischl, D. N. Greve, and L. L. Wald. "Identifying common-source driven correlations in resting-state fMRI via laminar-specific analysis in the human visual cortex". In: Stockholm, Sweden, 2010.

[158] M. Guidi, L. Huber, L. Lampe, and H. E. Moller. "Cortical laminar resting-state fluctuations scale with hypercapnic response”. In: 2016. 
[159] T. L. Davis, K. K. Kwong, R. M. Weisskoff, and B. R. Rosen. "Calibrated functional MRI: mapping the dynamics of oxidative metabolism”. In: Proceedings of the National academy of Sciences of the United States of America 95.4 (1998), pp. 1834-9.

[160] R. B. Buxton, K. Uludağ, D. J. Dubowitz, and T. T. Liu. "Modeling the hemodynamic response to brain activation". In: Neuroimage 23 Suppl 1 (2004), S220-33.

[161] S. Kashyap, D. Ivanov, M. Havlicek, B. A. Poser, and K. Uludağ. "High-resolution T -mapping $^{-}$ using inversion-recovery EPI and application to cortical depth-dependent fMRI at 7 Tesla”. In: Proceedings of the 24th Annual Meeting of ISMRM. Singapore, Republic of Singapore, 2016.

[162] E. Yacoub, K. Uğurbil, and N. Harel. "The spatial dependence of the poststimulus undershoot as revealed by high-resolution BOLD- and CBV-weighted fMRI". In: Journal of Cerebral Blood Flow and Metabolism 26.5 (2006), pp. 634-44.

[163] P. van Gelderen, X. Jiang, and J. H. Duyn. "Effects of magnetization transfer on T1 contrast in human brain white matter”. In: Neuroimage 128 (2016), pp. 85-95.

[164] H. Zeng and R. T. Constable. "Image distortion correction in EPI: comparison of field mapping with point spread function mapping”. In: Magnetic Resonance in Medicine 48.1 (2002), pp. 13746.

[165] L. Huber, M. Guidi, J. Goense, et al. "The magnitude point spread function is an inadequate measure of T2*-blurring in EPI”. In: vol. 23. 2015, pp. 2056-2056.

[166] L. Huber, J. Goense, A. J. Kennerley, et al. "Cortical lamina-dependent blood volume changes in human brain at 7 T”. In: Neuroimage 107 (2015), pp. 23-33.

[167] A. M. Puckett, K. M. Aquino, P. A. Robinson, M. Breakspear, and M. M. Schira. "The spatiotemporal hemodynamic response function for depth-dependent functional imaging of human cortex". In: Neuroimage 139 (2016), pp. 240-248.

[168] A. J. Kennerley, J. E. Mayhew, P. Redgrave, and J. Berwick. "Vascular Origins of BOLD and CBV fMRI Signals: Statistical Mapping and Histological Sections Compared”. In: Open Neuroimag $J$ 4 (2010), pp. 1-8.

[169] T. Krings, S. G. Erberich, F. Roessler, J. Reul, and A. Thron. "MR blood oxygenation leveldependent signal differences in parenchymal and large draining vessels: implications for functional MR imaging”. In: AJNR: American Journal of Neuroradiology 20.10 (1999), pp. 19071914.

[170] A. T. Lee, G. H. Glover, and C. H. Meyer. "Discrimination of large venous vessels in timecourse spiral blood-oxygen-level-dependent magnetic-resonance functional neuroimaging”. In: Magnetic Resonance in Medicine 33.6 (1995), pp. 745-54. 
[171] A. C. Silva and A. P. Koretsky. "Laminar specificity of functional MRI onset times during somatosensory stimulation in rat". In: Proceedings of the National academy of Sciences of the United States of America 99.23 (2002), pp. 15182-7.

[172] P. Tian, I. C. Teng, L. D. May, et al. "Cortical depth-specific microvascular dilation underlies laminar differences in blood oxygenation level-dependent functional MRI signal”. In: Proceedings of the National academy of Sciences of the United States of America 107.34 (2010), pp. 15246-51.

[173] L. Huber, J. Goense, A. J. Kennerley, et al. "Investigation of the neurovascular coupling in positive and negative BOLD responses in human brain at 7 T'. In: Neuroimage 97 (2014), pp. 349 62.

[174] Y. B. Sirotin, E. M. Hillman, C. Bordier, and A. Das. "Spatiotemporal precision and hemodynamic mechanism of optical point spreads in alert primates". In: Proceedings of the National academy of Sciences of the United States of America 106.43 (2009), pp. 18390-5.

[175] K. Uludağ. "To dip or not to dip: reconciling optical imaging and fMRI data". In: Proceedings of the National academy of Sciences of the United States of America 107.6 (2010), E23, author reply E24-E23, author reply E24.

[176] C. H. Moon, M. Fukuda, S. H. Park, and S. G. Kim. "Neural interpretation of blood oxygenation level-dependent fMRI maps at submillimeter columnar resolution”. In: Journal of Neuroscience 27.26 (2007), pp. 6892-902.

[177] L. Gagnon, S. Sakadzic, F. Lesage, et al. "Quantifying the microvascular origin of BOLD-fMRI from first principles with two-photon microscopy and an oxygen-sensitive nanoprobe”. In: Journal of Neuroscience 35.8 (2015), pp. 3663-75.

[178] R. S. Menon, S. Ogawa, D. W. Tank, and K. Uğurbil. "Tesla gradient recalled echo characteristics of photic stimulation-induced signal changes in the human primary visual cortex". In: Magnetic Resonance in Medicine 30.3 (1993), pp. 380-6.

[179] W. van der Zwaag, P. Buur, M. Versluis, and J. P. Marques. "Distortion-matched T1-maps and bias-corrected T1w-images as anatomical reference for submillimeter-resolution fMRI.” In: Proceedings of the 24th Annual Meeting of ISMRM. Singapore, Republic of Singapore, 2016.

[180] L. Huber, D. Ivanov, S. N. Krieger, et al. "Slab-selective, BOLD-corrected VASO at 7 Tesla provides measures of cerebral blood volume reactivity with high signal-to-noise ratio". In: Magnetic Resonance in Medicine 72.1 (2014), pp. 137-48.

[181] C. C. Yen, D. Papoti, and A. C. Silva. "Investigating the spatiotemporal characteristics of the deoxyhemoglobin-related and deoxyhemoglobin-unrelated functional hemodynamic response across cortical layers in awake marmosets”. In: Neuroimage 164 (2018), pp. 121-130. 
[182] T. van Mourik, J. P. J. M. van der Eerden, and D. Norris. "Laminar time course extraction over extended cortical areas”. In: Toronto, Canada, 2015.

[183] J. R. Polimeni, D. N. Greve, B. Fischl, and L. L. Wald. "Depth-resolved laminar analysis of resting-state fluctuation amplitude in high-resolution 7T fMRI”. In: Stockholm, Sweden, 2010.

[184] S. Kashyap, D. Ivanov, M. Havlicek, S. Sengupta, B. A. Poser, and K. Uludağ. "Resolving laminar activation in human V1 using ultra-high spatial resolution fMRI at 7 T". In: Scientific Reports 8.1 (2018), p. 17063.

[185] D.G. Amaral and P.L. Strick. “Ch. 15. The Organization of the Central Nervous System”. In: 2013.

[186] K. Zilles and K. Amunts. "Ch. 4. Anatomical Basis for Functional Specialization”. In: 30. 2015.

[187] C. D. Gilbert. “Ch. 25. The Constructive Nature of Visual Processing”. In: 2013.

[188] A. Shmuel and A. Maier. "Ch. 6. Locally Measured Neuronal Correlates of Functional MRI Signals". In: 30. 2015.

[189] D. H. Hubel and T. N. Wiesel. "Receptive fields, binocular interaction and functional architecture in the cat's visual cortex". In: The Journal of Physiology 160.1 (1962), pp. 106-154.

[190] A. Grinvald, E. Lieke, R. D. Frostig, C. D. Gilbert, and T. N. Wiesel. "Functional architecture of cortex revealed by optical imaging of intrinsic signals”. In: Nature 324.6095 (1986). Grinvald, A Lieke, E Frostig, R D Gilbert, C D Wiesel, T N eng EY05253/EY/NEI NIH HHS/ NS14716/NS/NINDS NIH HHS/ Research Support, U.S. Gov't, Non-P.H.S. Research Support, U.S. Gov't, P.H.S. England Nature. 1986 Nov 27-Dec 3;324(6095):361-4. doi: 10.1038/324361a0., pp. 361-4.

[191] K. Uğurbil. "What is feasible with imaging human brain function and connectivity using functional magnetic resonance imaging”. In: Philosophical Transactions of the Royal Society BBiological Sciences 371.1705 (2016). Dw2xp Times Cited:8 Cited References Count:119.

[192] M. E. Raichle. "The restless brain: how intrinsic activity organizes brain function". In: Philosophical Transactions of the Royal Society B-Biological Sciences 370.1668 (2015). Ch5jh Times Cited:57 Cited References Count:129, pp. 82-92.

[193] S. J. D. Lawrence, E. Formisano, L. Muckli, and F. P. de Lange. "Laminar fMRI: Applications for cognitive neuroscience". In: NeuroImage May (2017), pp. 1-7.

[194] S. O. Dumoulin, A. Fracasso, W. van der Zwaag, J. C. W. Siero, and N. Petridou. "Ultra-high field MRI: Advancing systems neuroscience towards mesoscopic human brain function”. In: Neuroimage 168 (2018), pp. 345-357. 
[195] I. Marquardt, M. Schneider, O. F. Gulban, D. Ivanov, and K. Uludag. "Cortical depth profiles of luminance contrast responses in human V1 and V2 using 7 T fMRI". In: Hum Brain Mapp (2018).

[196] J. W. Peirce. "Generating stimuli for neuroscience using PsychoPy". In: Frontiers in neuroinformatics 2 (2009), pp. 10-10.

[197] S. Sengupta, A. Roebroeck, V. G. Kemper, et al. "A Specialized Multi-Transmit Head Coil for High Resolution fMRI of the Human Visual Cortex at 7T". In: PLOS ONE 11.12 (2016), e0165418.

[198] A. Haase, J. Frahm, D. Matthaei, W. Hanicke, and K. D. Merboldt. "FLASH imaging. Rapid NMR imaging using low flip-angle pulses”. In: Journal of Magnetic Resonance (1969) 67.2 (1986), pp. 258-266.

[199] B. B. Avants, N. J. Tustison, J. Wu, P. A. Cook, and J. C. Gee. "An open source multivariate framework for n-tissue segmentation with evaluation on public data". In: Neuroinformatics 9.4 (2011), pp. 381-400.

[200] B. B. Avants, N. J. Tustison, G. Song, P. A. Cook, A. Klein, and J. C. Gee. "A reproducible evaluation of ANTs similarity metric performance in brain image registration". In: Neuroimage 54.3 (2011), pp. 2033-44.

[201] M. Jenkinson, C. F. Beckmann, T. E. J. Behrens, M. W. Woolrich, and S. M. Smith. "FSL". In: NeuroImage 62.2 (Aug. 2012), pp. 782-790.

[202] P. L. Bazin, M. Weiss, J. Dinse, A. Schafer, R. Trampel, and R. Turner. "A computational framework for ultra-high resolution cortical segmentation at 7Tesla”. In: Neuroimage 93 Pt 2.Part 2 (2014). Bazin, Pierre-Louis Weiss, Marcel Dinse, Juliane Schafer, Andreas Trampel, Robert Turner, Robert eng Research Support, Non-U.S. Gov't Neuroimage. 2014 Jun;93 Pt 2:201-9. doi: 10.1016/j.neuroimage.2013.03.077. Epub 2013 Apr 25., pp. 201-9.

[203] B. Dymerska, B. A. Poser, W. Bogner, et al. "Correcting dynamic distortions in 7T echo planar imaging using a jittered echo time sequence”. In: Magn Reson Med 76.5 (2016). 1522-2594 Dymerska, Barbara Poser, Benedikt A Bogner, Wolfgang Visser, Eelke Eckstein, Korbinian Cardoso, Pedro Barth, Markus Trattnig, Siegfried Robinson, Simon D KLI 264/Austrian Science Fund FWF/Austria Evaluation Studies Journal Article Research Support, Non-U.S. Gov’t United States Magn Reson Med. 2016 Nov;76(5):1388-1399. doi: 10.1002/mrm.26018. Epub 2015 Nov 19., pp. 1388-1399.

[204] J. Li, S. Chang, T. Liu, et al. "Reducing the object orientation dependence of susceptibility effects in gradient echo MRI through quantitative susceptibility mapping”. In: Magnetic Resonance in Medicine 68.5 (2012), pp. 1563-1569. 
[205] M. Moerel, F. De Martino, V. G. Kemper, et al. "Sensitivity and specificity considerations for fMRI encoding, decoding, and mapping of auditory cortex at ultra-high field". In: Neuroimage 164 (2018). 1095-9572 Moerel, Michelle De Martino, Federico Kemper, Valentin G Schmitter, Sebastian Vu, An T Ugurbil, Kamil Formisano, Elia Yacoub, Essa P30 NS076408/NS/NINDS NIH HHS/United States P41 EB015894/EB/NIBIB NIH HHS/United States S10 RR026783/RR/NCRR NIH HHS/United States Journal Article United States Neuroimage. 2018 Jan 1;164:18-31. doi: 10.1016/j.neuroimage.2017.03.063. Epub 2017 Mar 31., pp. 18-31.

[206] M. Zaitsev, B. Akin, P. LeVan, and B. R. Knowles. "Prospective motion correction in functional MRI”. In: Neuroimage 154 (2017). Zaitsev, Maxim Akin, Burak LeVan, Pierre Knowles, Benjamin R eng R01 DA021146/DA/NIDA NIH HHS/ Review Neuroimage. 2017 Jul 1;154:33-42. doi: 10.1016/j.neuroimage.2016.11.014. Epub 2016 Nov 11., pp. 33-42.

[207] S. Kashyap, D. Ivanov, M Havlicek, B. A. Poser, and K. Uludağ. "Laminar CBF and BOLD fMRI in the human visual cortex using arterial spin labelling at 7 T". In: Proceedings of the 27th Annual Meeting of ISMRM. Montréal, Canada, 2019.

[208] N. K. Logothetis and B. A. Wandell. "Interpreting the BOLD Signal”. In: Annu. Rev. Physiol. 66.1 (2004), pp. 735-769.

[209] S.-G. Kim. "Biophysics of BOLD fMRI investigated with animal models". In: Journal of Magnetic Resonance 292 (July 2018), pp. 82-89.

[210] L. L. Wald. "The future of acquisition speed, coverage, sensitivity, and resolution". In: Neuroimage 62.2 (2012), pp. 1221-9.

[211] B. Keil and L. L. Wald. "Massively parallel MRI detector arrays". In: Journal of Magnetic Resonance 229 (2013), pp. 75-89.

[212] P. A. Bandettini, R. Bowtell, P. Jezzard, and R. Turner. "Ultrahigh field systems and applications at $7 \mathrm{~T}$ and beyond: progress, pitfalls, and potential”. In: Magn Reson Med 67.2 (2012). Bandettini, Peter A Bowtell, Richard Jezzard, Peter Turner, Robert eng G0700399/Medical Research Council/United Kingdom G0901321/Medical Research Council/United Kingdom Z99 MH999999/NULL/Intramural NIH HHS/ ZIA MH002783-09/NULL/Intramural NIH HHS/ Comparative Study Magn Reson Med. 2012 Feb;67(2):317-21. doi: 10.1002/mrm.23151. Epub 2011 Nov 14., pp. 317-21.

[213] E. Formisano, D. S. Kim, F. Di Salle, P.-F. van de Moortele, K. Uğurbil, and R. Goebel. "Mirrorsymmetric tonotopic maps in human primary auditory cortex.” eng. In: Neuron 40 (4 Nov. 2003), pp. 859-69.

[214] M. I. Sereno, A. M. Dale, J. B. Reppas, et al. "Borders of multiple visual areas in humans revealed by functional magnetic resonance imaging.” eng. In: Science (New York, N.Y.) 268 (5212 May 1995), pp. 889-93. 
[215] S. O. Dumoulin and B. A. Wandell. "Population receptive field estimates in human visual cortex." eng. In: NeuroImage 39 (2 Jan. 2008), pp. 647-60.

[216] Wouter Schellekens, Natalia Petridou, and Nick F. Ramsey. "Detailed somatotopy in primary motor and somatosensory cortex revealed by Gaussian population receptive fields." eng. In: NeuroImage 179 (Oct. 2018), pp. 337-347.

[217] Wolfgang Grodd, Ernst Hülsmann, Martin Lotze, Dirk Wildgruber, and Michael Erb. "Sensorimotor mapping of the human cerebellum: fMRI evidence of somatotopic organization". In: Hum. Brain Mapp. 13.2 (Apr. 2001), pp. 55-73.

[218] M. W. Self, T. van Kerkoerle, R. Goebel, and P. R. Roelfsema. "Benchmarking laminar fMRI: Neuronal spiking and synaptic activity during top-down and bottom-up processing in the different layers of cortex". In: Neuroimage March (2017). Self, Matthew W van Kerkoerle, Timo Goebel, Rainer Roelfsema, Pieter R eng Review Neuroimage. 2017 Jun 23. pii: S1053-8119(17)30517-7. doi: 10.1016/j.neuroimage.2017.06.045., pp. 1-12.

[219] R. S. Menon, S. Ogawa, X. Hu, J. P. Strupp, P. Anderson, and K. Ugurbil. "BOLD based functional MRI at 4 Tesla includes a capillary bed contribution: echo-planar imaging correlates with previous optical imaging using intrinsic signals.” eng. In: Magnetic resonance in medicine 33 (3 Mar. 1995), pp. 453-9.

[220] R. Turner. "How much cortex can a vein drain? Downstream dilution of activation-related cerebral blood oxygenation changes.” eng. In: NeuroImage 16 (4 Aug. 2002), pp. 1062-7.

[221] G. Chen, F. Wang, J. C. Gore, and A. W. Roe. "Layer-specific BOLD activation in awake monkey V1 revealed by ultra-high spatial resolution functional magnetic resonance imaging”. In: Neuroimage 64.1 (2013), pp. 147-55.

[222] T. Jin and S.-G. Kim. "Cortical layer-dependent dynamic blood oxygenation, cerebral blood flow and cerebral blood volume responses during visual stimulation”. In: Neuroimage 43.1 (2008), pp. 1-9.

[223] F. Zhao, P. Wang, and S. G. Kim. “Cortical depth-dependent gradient-echo and spin-echo BOLD fMRI at 9.4T". In: Magnetic Resonance in Medicine 51.3 (2004), pp. 518-24.

[224] H. Lu, X. Golay, J. J. Pekar, and P. C. Van Zijl. "Sustained poststimulus elevation in cerebral oxygen utilization after vascular recovery". In: Journal of Cerebral Blood Flow and Metabolism 24.7 (2004), pp. 764-770.

[225] M. J. Donahue, H. Lu, C. K. Jones, R. A. E. Edden, J. J. Pekar, and P. C. M. van Zijl. “Theoretical and experimental investigation of the VASO contrast mechanism.” eng. In: Magnetic resonance in medicine 56 (6 Dec. 2006), pp. 1261-73. 
[226] E. C. Wong, R. B. Buxton, and L. R. Frank. "Implementation of quantitative perfusion imaging techniques for functional brain mapping using pulsed arterial spin labeling." eng. In: NMR in biomedicine 10 (4-5 June 1997), pp. 237-49.

[227] T. T. Liu and G. G. Brown. "Measurement of cerebral perfusion with arterial spin labeling: Part 1. Methods”. In: Journal of the International Neuropsychological Society 13.03 (Mar. 2007).

[228] T. Kim and S.-G. Kim. "Quantitative MRI of cerebral arterial blood volume”. In: Open Neuroimag $J 5$ (2011), pp. 136-45.

[229] S.-G. Kim, N. Harel, T. Jin, T. Kim, P. Lee, and F. Zhao. "Cerebral blood volume MRI with intravascular superparamagnetic iron oxide nanoparticles". In: NMR in Biomedicine 26 (2013), pp. 949-962.

[230] M. Fukuda, A. J. Poplawsky, and S. G. Kim. Submillimeter-resolution fMRI: Toward understanding local neural processing. Vol. 225. 2016, pp. 123-152.

[231] T. Q. Duong, D. S. Kim, K. Uğurbil, and S. G. Kim. "Localized cerebral blood flow response at submillimeter columnar resolution." eng. In: Proceedings of the National Academy of Sciences of the United States of America 98 (19 Sept. 2001), pp. 10904-9.

[232] T. Tjandra, J. C. W. Brooks, P. Figueiredo, R. Wise, P. M. Matthews, and I. Tracey. "Quantitative assessment of the reproducibility of functional activation measured with BOLD and MR perfusion imaging: implications for clinical trial design.” eng. In: NeuroImage 27 (2 Aug. 2005), pp. 393-401.

[233] M. Cavusoglu, A. Bartels, B. Yesilyurt, and K. Uludağ. "Retinotopic maps and hemodynamic delays in the human visual cortex measured using arterial spin labeling”. In: Neuroimage 59.4 (2012), pp. 4044-54.

[234] A. Gardumi, D. Ivanov, M. Havlicek, E. Formisano, and K. Uludağ. "Tonotopic maps in human auditory cortex using arterial spin labeling”. In: Human Brain Mapping (2016).

[235] D. C. Alsop, J. A. Detre, X. Golay, et al. "Recommended implementation of arterial spin-labeled perfusion MRI for clinical applications: A consensus of the ISMRM perfusion study group and the European consortium for ASL in dementia.” eng. In: Magnetic resonance in medicine 73 (1 Jan. 2015), pp. 102-16.

[236] W. D. Rooney, G. Johnson, X. Li, et al. "Magnetic field and tissue dependencies of human brain longitudinal 1H2O relaxation in vivo." eng. In: Magnetic resonance in medicine 57 (2 Feb. 2007), pp. 308-18. 
[237] P. J. Wright, O. E. Mougin, J. J. Totman, et al. "Water proton T1 measurements in brain tissue at 7, 3, and 1.5 T using IR-EPI, IR-TSE, and MPRAGE: results and optimization.” eng. In: Magma (New York, N.Y.) 21 (1-2 Mar. 2008), pp. 121-30.

[238] D. Ivanov, S. Kashyap, R. A. M. Haast, et al. "Whole-brain sub-millimeter isotropic resolution cerebral blood flow map in humans". In: Proceedings of the 26th Annual Meeting of ISMRM. Paris, France, 2018.

[239] A.G. Webb. "Dielectric materials in magnetic resonance". In: Concepts in Magnetic Resonance Part A 38A.4 (2011), pp. 148-184.

[240] K. Haines, N. B. Smith, and A. G. Webb. "New high dielectric constant materials for tailoring the B1+ distribution at high magnetic fields". In: Journal of Magnetic Resonance 203.2 (Apr. 2010), pp. 323-327.

[241] W. M. Teeuwisse, W. M. Brink, and A. G. Webb. "Quantitative assessment of the effects of highpermittivity pads in 7 Tesla MRI of the brain.” In: Magnetic resonance in medicine 67 (5 May 2012), pp. 1285-93.

[242] K. K. Kwong, D. A. Chesler, R. M. Weisskoff, et al. "MR perfusion studies with T1-weighted echo planar imaging”. In: Magnetic Resonance in Medicine 34.6 (Dec. 1995), pp. 878-887.

[243] S.-G. Kim and N. V. Tsekos. "Perfusion imaging by a flow-sensitive alternating inversion recovery (Fair) technique: Application to functional brain imaging”. In: Magnetic Resonance in Medicine 37.3 (Mar. 1997), pp. 425-435.

[244] E. C. Wong, R. B. Buxton, and L. R. Frank. "Quantitative imaging of perfusion using a single subtraction (QUIPSS and QUIPSS II).” eng. In: Magnetic resonance in medicine 39 (5 May 1998), pp. 702-8.

[245] S. M. Smith. "Fast robust automated brain extraction." eng. In: Human brain mapping 17 (3 Nov. 2002), pp. 143-55.

[246] B. Fischl. "FreeSurfer”. In: NeuroImage 62.2 (Aug. 2012), pp. 774-781.

[247] L. Wang, R. E. Mruczek, M. J. Arcaro, and S. Kastner. "Probabilistic Maps of Visual Topography in Human Cortex”. In: Cereb Cortex 25.10 (2015), pp. 3911-31.

[248] N. C. Benson, O. H. Butt, D. H. Brainard, and G. K. Aguirre. "Correction of Distortion in Flattened Representations of the Cortical Surface Allows Prediction of V1-V3 Functional Organization from Anatomy”. In: PLoS Computational Biology 10.3 (Mar. 2014). Ed. by Wolfgang Einhäuser, e1003538.

[249] K. J. Worsley. "Statistical analysis of activation images". In: Functional MRI: An introduction to methods 14 (2001), pp. 251-270. 
[250] K. Kay, K. W. Jamison, L. Vizioli, R. Zhang, E. Margalit, and K. Uğurbil. "A critical assessment of data quality and venous effects in sub-millimeter fMRI”. In: Neuroimage 189 (Apr. 2019), pp. 847-869.

[251] G. K. Aguirre, J. A. Detre, E. Zarahn, and D. C. Alsop. "Experimental design and the relative sensitivity of BOLD and perfusion fMRI”. In: Neuroimage 15.3 (2002), pp. 488-500.

[252] K. Wagstyl, C. Paquola, R. Bethlehem, A. C. Evans, and A. Huth. Equivolumetric layering for mesh surfaces. Oct. 2018. URL: https://doi.org/10.5281/zenodo.1442584.

[253] Jiongjiong Wang, David C. Alsop, Hee Kwon Song, et al. "Arterial transit time imaging with flow encoding arterial spin tagging (FEAST)." eng. In: Magnetic resonance in medicine 50 (3 Sept. 2003), pp. 599-607.

[254] T. Q. Duong, D. S. Kim, K. Uğurbil, and S. G. Kim. "Spatiotemporal dynamics of the BOLD fMRI signals: Toward mapping submillimeter cortical columns using the early negative response”. In: Magnetic Resonance in Medicine 44.2 (2000), pp. 231-242.

[255] T. Kim and S.-G. Kim. "Cortical layer-dependent arterial blood volume changes: Improved spatial specificity relative to BOLD fMRI". In: NeuroImage 49.2 (Jan. 2010), pp. 1340-1349.

[256] J. Berwick, D. Johnston, M. Jones, et al. "Fine Detail of Neurovascular Coupling Revealed by Spatiotemporal Analysis of the Hemodynamic Response to Single Whisker Stimulation in Rat Barrel Cortex". In: Journal of Neurophysiology 99.2 (May 2008), pp. 787-798.

[257] D. Kurban, G. Liberman, S. Kashyap, D. Ivanov, and B. A. Poser. "Dual-echo simultaneous multislice spiral acquisition for simultaneous CBF and BOLD fMRI at 7T". In: Proceedings of the 27th Annual Meeting of ISMRM. Montréal, Canada, 2019.

[258] M. Engel, L. Kasper, C. Barmet, et al. "Single-shot spiral imaging at 7 T." eng. In: Magnetic resonance in medicine 80 (5 Nov. 2018), pp. 1836-1846.

[259] S. Aslan, F. Xu, P. L. Wang, et al. "Estimation of labeling efficiency in pseudocontinuous arterial spin labeling.” eng. In: Magnetic resonance in medicine 63 (3 2010), pp. 765-71.

[260] W. M. Luh, S. L. Talagala, T. Q. Li, and P. A. Bandettini. "Pseudo-continuous arterial spin labeling at $7 \mathrm{~T}$ for human brain: estimation and correction for off-resonance effects using a Prescan". eng. In: Magnetic resonance in medicine 69.22488568 (Feb. 2013), pp. 402-410.

[261] A. Alsaedi, D. Thomas, S. Bisdas, and X. Golay. "Overview and Critical Appraisal of Arterial Spin Labelling Technique in Brain Perfusion Imaging”. In: Contrast Media Mol Imaging 2018 (2018), p. 15. 
[262] M. G. Mora Álvarez, R. W. Stobbe, and C. Beaulieu. "High resolution continuous arterial spin labeling of human cerebral perfusion using a separate neck tagging RF coil”. In: PLOS ONE 14.4 (Apr. 2019).

[263] N. Petridou, M. Italiaander, B. L. van de Bank, J. C. W. Siero, P. R. Luijten, and D. W. J. Klomp. "Pushing the limits of high-resolution functional MRI using a simple high-density multi-element coil design". In: NMR Biomed. 26.1 (Apr. 2013), pp. 65-73.

[264] A. J. W. van der Kouwe, T. Benner, D. H. Salat, and B. Fischl. "Brain morphometry with multiecho MPRAGE.” eng. In: NeuroImage 40 (2 Apr. 2008), pp. 559-569.

[265] N. J. Tustison, B. B. Avants, P. A. Cook, et al. “N4ITK: improved N3 bias correction.” eng. In: IEEE transactions on medical imaging 29 (6 June 2010), pp. 1310-20.

[266] A. Lutti, D. L. Thomas, C. Hutton, and N. Weiskopf. "High-resolution functional MRI at 3 T: 3D/2D echo-planar imaging with optimized physiological noise correction”. eng. In: Magnetic resonance in medicine 69.22821858 (2013), pp. 1657-1664.

[267] W. van der Zwaag, P. F. Buur, A. Fracasso, et al. "Distortion-matched T1 maps and unbiased T1weighted images as anatomical reference for high-resolution fMRI”. In: NeuroImage 176 (Aug. 2018), pp. 41-55.

[268] R. B. Buxton, E. C. Wong, and L. R. Frank. "Dynamics of blood flow and oxygenation changes during brain activation: the balloon model”. In: Magnetic Resonance in Medicine 39.6 (1998), pp. $855-64$.

[269] K. J. Friston, L. Harrison, and W. Penny. "Dynamic causal modelling”. In: Neuroimage 19.4 (2003), pp. 1273-302.

[270] M. Havlicek and K. Uludağ. "A dynamical model of the laminar BOLD response”. In: bioRxiv (Jan. 2019), p. 609099.

[271] R. B. Buxton. “The elusive initial dip”. In: Neuroimage 13.6 Pt 1 (2001), pp. 953-8.

[272] E. S. Finn, L. Huber, D. C. Jangraw, and P. A. Bandettini. "Layer-dependent activity in human prefrontal cortex during working memory”. In: bioRxiv (Jan. 2018), p. 425249.

[273] D. Ivanov, B. A. Poser, S. Kashyap, A. Gardumi, L. Huber, and K. Uludağ. "Sub-millimeter human brain perfusion imaging using arterial spin labelling at 3 and 7 Tesla”. In: ISMRM Workshop on Ultra High Field MRI. Heidelberg, Germany, 2016.

[274] S. Kashyap, L. Huber, D. Ivanov, K. Uludağ, D. A. Feinberg, and B. A. Poser. "Ultra-High Spatial Resolution Multi-Echo BOLD \& VASO fMRI of the Human Motor Cortex at 9.4 T". In: ISMRM Workshop on Ultra High Field MRI. Dubrovnik, Croatia, 2019. 
[275] M. Balasubramanian, R. V. Mulkern, S. E. Maier, and J. R. Polimeni. "Tangential and radial diffusion in human primary somatosensory and motor cortex: evidence from in-vivo line-scan acquisitions at 7T with 250-500 micron radial resolution”. In: Proceedings of the 27th Annual Meeting of ISMRM. Montréal, Canada, 2019.

[276] J. C. W. Siero, I. A. F. de Oliveira, S. Choi, and X. Yu. "Implementing human line-scanning fMRI: Initial results of ultra-high temporal and spatial resolution fMRI". In: Proceedings of the 27th Annual Meeting of ISMRM. Montréal, Canada, 2019.

[277] M. Havlicek, A. Roebroeck, K. Friston, A. Gardumi, D. Ivanov, and K. Uludağ. "Physiologically informed dynamic causal modeling of fMRI data”. In: Neuroimage 122 (2015), pp. 355-72.

[278] S. Kashyap, F. J. Fritz, R. L. Harms, et al. "Effect of optimised coil-combinations on highresolution laminar fMRI at 9.4T". In: Proceedings of the 26th Annual Meeting of ISMRM. Paris, France, 2018.

[279] Kashyap, S., D. Ivanov, M. Havlicek, B. A. Poser, and K. Uludağ. "Impact of acquisition and analysis strategies on cortical depth-dependent fMRI". In: Neuroimage 168 (2018), pp. 332-344.

[280] Kashyap, S., D. Ivanov, M. Havlicek, S. Sengupta, B. A. Poser, and K. Uludağ. "Resolving laminar activation in human V1 using ultra-high spatial resolution fMRI at 7 T". In: Scientific Reports 8.1 (2018), p. 17063.

[281] Kashyap, S., D. Ivanov, M Havlicek, B. A. Poser, and K. Uludağ. "Laminar CBF and BOLD fMRI in the human visual cortex using arterial spin labelling at 7 T". In: in prep. (2019).

[282] L. Huber, D. H. Y. Tse, C. J. Wiggins, et al. "Ultra-high resolution blood volume fMRI and BOLD fMRI in humans at 9.4T: Capabilities and challenges". In: Neuroimage 178 (2018), pp. 769-779.

[283] L. Huber, E. S. Finn, D. A. Handwerker, et al. "Sub-millimeter fMRI reveals multiple topographical digit representations that form action maps in human motor cortex”. In: bioRxiv (Jan. 2019), p. 457002.

[284] Kashyap, S., D. Ivanov, M. Havlicek, B. A. Poser, and K. Uludağ. "High-resolution T 1 -mapping using inversion-recovery EPI and application to cortical depth-dependent fMRI at 7 Tesla”. In: Proceedings of the Benelux Chapter of the ISMRM. Eindhoven, The Netherlands, 2016.

[285] Kashyap, S., D. Ivanov, M. Havlicek, B. A. Poser, and K. Uludağ. "High-resolution T 1 -mapping using inversion-recovery EPI and application to cortical depth-dependent fMRI at 7 Tesla”. In: Proceedings of the 24th Annual Meeting of ISMRM. Singapore, Republic of Singapore, 2016.

[286] Kashyap, S., D. Ivanov, M. Havlicek, S. Sengupta, B. A. Poser, and K. Uludağ. "True laminar resolution fMRI of the human visual cortex at 7 T". summa cum laude. In: Proceedings of the 26th Annual Meeting of ISMRM. Paris, France, 2018. 
[287] Kashyap, S., D. Ivanov, M Havlicek, B. A. Poser, and K. Uludağ. "Laminar CBF and BOLD fMRI in the human visual cortex using arterial spin labelling at 7 T". magna cum laude. In: Proceedings of the 27th Annual Meeting of ISMRM. Montréal, Canada, 2019.

[288] Kashyap, S. "Functional neuroimaging using MRI". In: Faculty of Arts and Social Sciences. Maastricht University. Maastricht, The Netherlands, 2018.

[289] Kashyap, S. "Acquisition and analysis strategies for laminar fMRI at ultra-high field." In: Brain-In-Depth Symposium (BIDS). Max Planck Institute for Human Cognitive and Brain Sciences. Leipzig, Germany, 2019.

[290] Kashyap, S. "Neurovascular Coupling”. In: MBIC Graduate School Course on BOLD Physiology. Maastricht University. Maastricht, The Netherlands, 2019.

[291] Kashyap, S., D. Ivanov, M. Havlicek, B. A. Poser, and K. Uludağ. "Sub-millimetre T 1 -mapping using inversion-recovery EPI and application to cortical depth-dependent fMRI at 7 Tesla". In: ISMRM Workshop on Ultra High Field MRI. Heidelberg, Germany, 2016.

[292] Kashyap, S., F. J. Fritz, R. L. Harms, et al. "Effect of optimised coil-combinations on highresolution laminar fMRI at 9.4T”. In: Proceedings of the 26th Annual Meeting of ISMRM. Paris, France, 2018.

[293] Kashyap, S., D. Ivanov, M. Havlicek, S. Sengupta, B. A. Poser, and K. Uludağ. "True laminar resolution fMRI of the human visual cortex at 7 T'. In: Brain-in-depth Symposium (BIDS). Magdeburg, Germany, 2018.

[294] Kashyap, S., L. Huber, D. Ivanov, K. Uludağ, D. A. Feinberg, and B. A. Poser. "Ultra-High Spatial Resolution Multi-Echo BOLD \& VASO fMRI of the Human Motor Cortex at 9.4 T". In: ISMRM Workshop on Ultra High Field MRI. Dubrovnik, Croatia, 2019.

[295] T. Christofer, K. Mahati, V. Bhagya, et al. "Amygdalar inactivation prevents stress-induced cognitive deficits, anxiety behavior and impaired hippocampal long-term potentiation”. In: Society for Neuroscience. Washington D. C., USA, 2011, pp. 719.10/XX9.

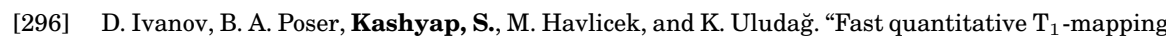
at ultra-high field using inversion-recovery echo planar imaging”. In: Proceedings of the OHBM. Honolulu, USA, 2015.

[297] D. Ivanov, B. A. Poser, Kashyap, S., A. Gardumi, L. Huber, and K. Uludağ. "Sub-millimeter human brain perfusion imaging using arterial spin labelling at 3 and 7 Tesla". In: ISMRM Workshop on Ultra High Field MRI. Heidelberg, Germany, 2016.

[298] M. Havlicek, Kashyap, S., D. Ivanov, and K. Uludağ. "Towards an optimal analysis of laminarresolved fMRI". In: Proceedings of the OHBM. Geneva, Switzerland, 2016. 
[299] J. Reithler, Kashyap, S., D. Ivanov, and R. Goebel. "Quantitative in vivo assessment of human hippocampal subfield tissue properties at 7T”. In: Proceedings of the OHBM. Vancouver, Canada, 2017.

[300] L. Huber, D. H. Y. Tse, Kashyap, S., et al. "Ultra-high resolution blood volume fMRI and BOLD fMRI in humans at 9.4T: Capabilities and challenges". In: Proceedings of the 25th Annual Meeting of the ISMRM. Honolulu, USA, 2017.

[301] D. Ivanov, Kashyap, S., R. A. M. Haast, et al. "Whole-brain sub-millimeter isotropic resolution cerebral blood flow map in humans". In: Proceedings of the 26th Annual Meeting of ISMRM. Paris, France, 2018.

[302] L. Huber, J. Goense, P. Molfese, et al. "Functional mapping of body part representations across layers and columns in humans”. In: Proceedings of the 26th Annual Meeting of ISMRM. Paris, France, 2018.

[303] D. Kurban, G. Liberman, Kashyap, S., D. Ivanov, and B. A. Poser. "Simultaneous multi-slice spiral acquisitions for CBF fMRI at 7T". In: ISMRM Workshop on Ultra High Field MRI. Dubrovnik, Croatia, 2019.

[304] L. Huber, E. Merriam, I. Kim, et al. "Measuring layer-dependent CBV fMRI in the visual system”. In: Proceedings of the 27th Annual Meeting of ISMRM. Montréal, Canada, 2019.

[305] D. Kurban, G. Liberman, Kashyap, S., D. Ivanov, and B. A. Poser. "Dual-echo simultaneous multi-slice spiral acquisition for simultaneous CBF and BOLD fMRI at 7T". In: Proceedings of the 27th Annual Meeting of ISMRM. Montréal, Canada, 2019.

[306] R. A. M. Haast, D. Ivanov, J. DeKraker, et al. "Sub-millimeter blood flow mapping of cortical and hippocampal gray matter". In: Proceedings of the 27th Annual Meeting of ISMRM. Montréal, Canada, 2019. 



\section{Valorisation}

The work presented in this thesis describes important methodological developments that are not directly valorisable (making knowledge available for economic and/or societal use) but are of value to scientists pursuing basic research and methods developers. The work presented here can be seen as building blocks for applications that can create wider societal and economic impact. These applications will also stand to benefit from the recent adoption of ultra-high field MRI (7 T) into the clinical environment. Under these caveats, the valorisable aspects of the work presented in the thesis are described in this addendum.

Over the past couple of decades, Magnetic Resonance Imaging (MRI) technology has permeated the societal landscape having had enormous impact in the progress of diagnostic healthcare and modern medicine. MRI has achieved mainstream adoption being the tool of choice for imaging the internal structures of the human body such as the soft tissue, blood vessels, etc. in health and disease. In the neurosciences, however, neurological or psychiatric conditions (such as pain, depression etc.) are not easily associated with readily visible structural abnormalities (such as tumours, lesions etc.). One way to address this is to use methods that allow detection of brain function. Until the early 1990 s, the only way to achieve mapping of brain function was using radiolabelled tracer molecules that can respond to changes in blood flow or glucose consumption, and measuring these changes using positron emission tomography (PET) or single photon emission computed tomography (SPECT). MRI-based functional mapping, on the other hand, is noninvasive and is considered to be a safe technology due to the absence of ionising 
radiation and radioactive tracers.

Functional MRI (fMRI) typically uses an intrinsic contrast mechanism that is sensitive to local changes in blood-oxygenation (blood oxygenation level dependent, BOLD) during brain activity, proving a rapid and safe method to assess brain function. While the early fMRI efforts were focussed on mapping different brain areas ("functional corticography"), the availability of ultra-high field MRI systems $(\geq 7 \mathrm{~T}$ ) has ushered in a new era for functional neuroimaging in humans enabling fast, high-resolution, acquisitions that permit precise localisation and allow mapping of sub-areas of the brain. Despite all the advances in ultrahigh field, high-resolution fMRI research and technology, fMRI has not taken the clinic by storm, making it difficult to "valorise" in the sense of direct commercial or industrial impact. Nevertheless, there is no doubt that MRI is the tool that could shed more light on different aspects of living human brain and pave the way to clinical treatments. The potential usefulness of fMRI for clinical populations has been best described in a fantastic blogpost by Prof. Dr. Peter Bandettini ${ }^{1}$. Among the issues raised for the lack of clinical adoption is that fMRI requires several intermediate data processing steps to become interpretable (this is true at all field strengths). At ultra-high field, the complexity of these strategies is compounded by the sheer size of the data being acquired, resulting in increased computational time and requiring computational, data analysis expertise that often falling outside the purview of even a trained Radiologist. Therefore, effective data processing and analysis strategies could turn the tide for potential clinical applications of fMRI. Specifically, the work presented in this thesis describe novel data acquisition, processing and analysis strategies for high-resolution fMRI targeted towards investigating the mesoscale $(0.5-1.0 \mathrm{~mm})$ architecture of the human brain

\footnotetext{
${ }^{1}$ BrainBlog: If, how, and when fMRI goes clinical
} 
and therefore, has an inherent "value" to field beyond the application to laminar fMRI.

While many existing MRI tools are optimised for whole-brain datasets, higher spatial resolution data often only cover a region of the brain one is investigating. The work presented in this thesis presents approaches and algorithms that can be used to deal with partial coverage (f)MRI data. A stumbling block in MRI research is the making novel MRI sequences across sites and vendors. There are several MRI vendors, e.g. Philips, Siemens, but work with different software ecosystems for programming the techniques. For example, Chapter 2 of this thesis emphasises the value of acquiring anatomical data that are distortion-matched to the functional data. Adoption of this approach is currently only limited by the fact that different ultra-high field scanners are operating on different software versions requiring considerable reprogramming of the sequence. This is notwithstanding the fact that the underlying physics is the same. The distortion-matched anatomical acquisition scheme used in the Chapter 2 was recently programmed in a Philips environment by van der Zwaag and colleagues. Hopefully, this will be easier in the near future thanks to recent progrses in open-source pulse sequence development frameworks such as Pulseq ${ }^{2}$. The open-source frameworks allow the programming of MRI pulse sequences outside the vendor-controlled environment and with little effort by sequence programmer, prepares them to be executed on any scanner.

The laminar fMRI acquisition strategy presented in Chapter 3 was inspired by a technique in animal research called line-scanning fMRI [79] that allows both high spatial and temporal resolution for mapping laminar activity in a small patch of the cortex. While it may seem a niche application, the interest in line-scanning-

\footnotetext{
${ }^{2}$ https://pulseq.github.io/
} 
like human laminar fMRI has since then increased with many sites successfully pickup the baton [275, 276]. Line-scanning-like fMRI in humans creates value in allowing us to investigate the cortical micro-circuitry in humans using $7 \mathrm{~T}$ MRI at nearly the resolution of the cortical layers themselves. There is further value in bridging the gap between invasive animal and non-invasive human research and possibly pave the way for research to be more translatable.

Other valorisable aspects are the technical and educational outcomes of the work presented in this thesis. There are algorithms and analyses packages freely available (such as FSL, SPM) that can be used for fMRI data processing. However, many of these tools have been developed to work with data acquired at field strengths of $3 \mathrm{~T}$, typically for whole brain acquisitions at low spatial resolutions (2-3 mm). Given that the default parameters are sub-optimal for higher resolution $7 \mathrm{~T}$ data, the availability of the source code for these tools in the public domain has been absolutely critical in improving one's understanding of the algorithms and guide one's choices as to what parameters best suit the data they are working with. Furthermore, being an unabashed proponent of fair and open collaboration, the course of the present $\mathrm{PhD}$ has yielded active engagement with peers in the department and those at conferences sharing information without reservation. An example of this shared value, is an electronic poster presented at the annual meeting of the International Society for Magnetic Resonance in Medicine (ISMRM) 2018 [278]. Neuroimaging blogs practicalfmri.blogspot.com and www. layerfmri.com have been a great example of knowledge transfer and value sharing with respect to the practical aspects of fMRI research. An opportunity to contribute to the value sharing was afforded thanks to the author of www. layerfmri.com and has made it possible to describe some of the data processing strategies discussed in Chapters 4-5, in more practical detail than would possible 
in a corresponding publication.

Lastly and arguably most importantly to some people, that the scripts and workflows developed over the course of the present work will be soon available online ${ }^{3}$ as educational scripts (e.g. MATLAB LiveScript, R Markdown). In conclusion, it is important to underline the fact that the valorisation potential of even basic/fundamental research can be expanded by ensuring the knowledge generated is made available in an open and accessible way. Moreover, making the knowledge accessible can increase opportunities for collaboration and therefore, create value for the society through scientific progress, as a collective.

\footnotetext{
${ }^{3}$ Gitlab repository
} 



\section{Research output}

\section{Articles}

[1] Kashyap, S., D. Ivanov, M. Havlicek, B. A. Poser, and K. Uludağ. "Impact of acquisition and analysis strategies on cortical depth-dependent fMRI”. in: Neuroimage 168 (2018), pp. 332-344

[2] Kashyap, S., D. Ivanov, M. Havlicek, S. Sengupta, B. A. Poser, and K. Uludağ. "Resolving laminar activation in human V1 using ultra-high spatial resolution fMRI at 7 T'. in: Scientific Reports 8.1 (2018), p. 17063

[3] Kashyap, S., D. Ivanov, M Havlicek, B. A. Poser, and K. Uludağ. "Laminar CBF and BOLD fMRI in the human visual cortex using arterial spin labelling at 7 T". in: in prep. (2019)

\section{Co-authored}

[1] L. Huber, D. H. Y. Tse, C. J. Wiggins, K. Uludağ, Kashyap, S., D. C. Jangraw, P. A. Bandettini, B. A. Poser, and D. Ivanov. "Ultra-high resolution blood volume fMRI and BOLD fMRI in humans at 9.4T: Capabilities and challenges”. In: Neuroimage 178 (2018), pp. 769-779

[2] L. Huber, E. S. Finn, D. A. Handwerker, M. Boenstrup, D. Glen, Kashyap, S., D. Ivanov, N. Petridou, S. Marrett, J. Goense, B. A. Poser, and P. A. Bandettini. "Sub-millimeter fMRI reveals multiple topographical digit representations that form action maps in human motor cortex". In: bioRxiv (Jan. 2019), p. 457002

\section{Conference talks}

[1] Kashyap, S., D. Ivanov, M. Havlicek, B. A. Poser, and K. Uludağ. "High-resolution $\mathrm{T}_{1}$-mapping using inversion-recovery EPI and application to cortical depth-dependent fMRI at 7 Tesla”. In: Proceedings of the Benelux Chapter of the ISMRM. Eindhoven, The Netherlands, 2016

[2] Kashyap, S., D. Ivanov, M. Havlicek, B. A. Poser, and K. Uludağ. "High-resolution T $_{1}$-mapping using inversion-recovery EPI and application to cortical depth-dependent fMRI at 7 Tesla”. In: Proceedings of the 24th Annual Meeting of ISMRM. Singapore, Republic of Singapore, 2016 
[3] Kashyap, S., D. Ivanov, M. Havlicek, S. Sengupta, B. A. Poser, and K. Uludağ. "True laminar resolution fMRI of the human visual cortex at 7 T". summa cum laude. In: Proceedings of the 26th Annual Meeting of ISMRM. Paris, France, 2018

[4] Kashyap, S., D. Ivanov, M Havlicek, B. A. Poser, and K. Uludağ. "Laminar CBF and BOLD fMRI in the human visual cortex using arterial spin labelling at $7 \mathrm{~T}$ ". magna cum laude. In: Proceedings of the 27th Annual Meeting of ISMRM. Montréal, Canada, 2019

\section{Educational and invited talks}

[1] Kashyap, S. "Functional neuroimaging using MRI". in: Faculty of Arts and Social Sciences. Maastricht University. Maastricht, The Netherlands, 2018

[2] Kashyap, S. "Acquisition and analysis strategies for laminar fMRI at ultra-high field." In: BrainIn-Depth Symposium (BIDS). Max Planck Institute for Human Cognitive and Brain Sciences. Leipzig, Germany, 2019

[3] Kashyap, S. "Neurovascular Coupling”. In: MBIC Graduate School Course on BOLD Physiology. Maastricht University. Maastricht, The Netherlands, 2019

\section{Conference Proceedings}

[1] Kashyap, S., D. Ivanov, M. Havlicek, B. A. Poser, and K. Uludağ. "Sub-millimetre T 1 -mapping using inversion-recovery EPI and application to cortical depth-dependent fMRI at 7 Tesla”. In: ISMRM Workshop on Ultra High Field MRI. Heidelberg, Germany, 2016

[2] Kashyap, S., D. Ivanov, M. Havlicek, B. A. Poser, and K. Uludağ. "High-resolution T 1 -mapping using inversion-recovery EPI and application to cortical depth-dependent fMRI at 7 Tesla". In: Proceedings of the 24th Annual Meeting of ISMRM. Singapore, Republic of Singapore, 2016

[3] Kashyap, S., D. Ivanov, M. Havlicek, S. Sengupta, B. A. Poser, and K. Uludağ. "True laminar resolution fMRI of the human visual cortex at 7 T". summa cum laude. In: Proceedings of the 26th Annual Meeting of ISMRM. Paris, France, 2018

[4] Kashyap, S., F. J. Fritz, R. L. Harms, L. Huber, D. Ivanov, A. Roebroeck, B. A. Poser, and K. Uludağ. "Effect of optimised coil-combinations on high-resolution laminar fMRI at 9.4T". in: Proceedings of the 26th Annual Meeting of ISMRM. Paris, France, 2018 
[5] Kashyap, S., D. Ivanov, M. Havlicek, S. Sengupta, B. A. Poser, and K. Uludağ. “True laminar resolution fMRI of the human visual cortex at 7 T". in: Brain-in-depth Symposium (BIDS). Magdeburg, Germany, 2018

[6] Kashyap, S., L. Huber, D. Ivanov, K. Uludağ, D. A. Feinberg, and B. A. Poser. "Ultra-High Spatial Resolution Multi-Echo BOLD \& VASO fMRI of the Human Motor Cortex at 9.4 T". in: ISMRM Workshop on Ultra High Field MRI. Dubrovnik, Croatia, 2019

[7] Kashyap, S., D. Ivanov, M Havlicek, B. A. Poser, and K. Uludağ. "Laminar CBF and BOLD fMRI in the human visual cortex using arterial spin labelling at 7 T". magna cum laude. In: Proceedings of the 27th Annual Meeting of ISMRM. Montréal, Canada, 2019

\section{Co-authored}

[1] T. Christofer, K. Mahati, V. Bhagya, T. Kuldeep, Sriranga, K., S. Praveena, T. R. Raju, and B. S. Shankaranarayana Rao. "Amygdalar inactivation prevents stress-induced cognitive deficits, anxiety behavior and impaired hippocampal long-term potentiation". In: Society for Neuroscience. Washington D. C., USA, 2011, pp. 719.10/XX9

[2] D. Ivanov, B. A. Poser, Kashyap, S., M. Havlicek, and K. Uludağ. "Fast quantitative T -mapping $^{-}$ at ultra-high field using inversion-recovery echo planar imaging”. In: Proceedings of the OHBM. Honolulu, USA, 2015

[3] D. Ivanov, B. A. Poser, Kashyap, S., A. Gardumi, L. Huber, and K. Uludağ. "Sub-millimeter human brain perfusion imaging using arterial spin labelling at 3 and 7 Tesla". In: ISMRM Workshop on Ultra High Field MRI. Heidelberg, Germany, 2016

[4] M. Havlicek, Kashyap, S., D. Ivanov, and K. Uludağ. "Towards an optimal analysis of laminarresolved fMRI". in: Proceedings of the OHBM. Geneva, Switzerland, 2016

[5] J. Reithler, Kashyap, S., D. Ivanov, and R. Goebel. "Quantitative in vivo assessment of human hippocampal subfield tissue properties at 7T". in: Proceedings of the OHBM. Vancouver, Canada, 2017

[6] L. Huber, D. H. Y. Tse, Kashyap, S., C. J. Wiggins, K. Uludağ, P. A. Bandettini, B. A. Poser, and D. Ivanov. "Ultra-high resolution blood volume fMRI and BOLD fMRI in humans at 9.4T: Capabilities and challenges". In: Proceedings of the 25th Annual Meeting of the ISMRM. Honolulu, USA, 2017 
[7] D. Ivanov, Kashyap, S., R. A. M. Haast, S. Janssens, L. Huber, B. A. Poser, and K. Uludağ. "Whole-brain sub-millimeter isotropic resolution cerebral blood flow map in humans". In: Proceedings of the 26th Annual Meeting of ISMRM. Paris, France, 2018

[8] L. Huber, J. Goense, P. Molfese, D. Glen, D. Jangraw, Kashyap, S., B. A. Poser, D. A. Handwerker, and P. A. Bandettini. "Functional mapping of body part representations across layers and columns in humans". In: Proceedings of the 26th Annual Meeting of ISMRM. Paris, France, 2018

[9] D. Kurban, G. Liberman, Kashyap, S., D. Ivanov, and B. A. Poser. "Simultaneous multi-slice spiral acquisitions for CBF fMRI at 7T". in: ISMRM Workshop on Ultra High Field MRI. Dubrovnik, Croatia, 2019

[10] L. Huber, E. Merriam, I. Kim, Y. Chai, Kashyap, S., J. Polimeni, Z. Roth, W.-M. Shim, S.-G. Kim, D. Ivanov, B. A. Poser, and P. A. Bandettini. "Measuring layer-dependent CBV fMRI in the visual system". In: Proceedings of the 27th Annual Meeting of ISMRM. Montréal, Canada, 2019

[11] D. Kurban, G. Liberman, Kashyap, S., D. Ivanov, and B. A. Poser. "Dual-echo simultaneous multi-slice spiral acquisition for simultaneous CBF and BOLD fMRI at 7T". in: Proceedings of the 27th Annual Meeting of ISMRM. Montréal, Canada, 2019

[12] R. A. M. Haast, D. Ivanov, J. DeKraker, Kashyap, S., S. E. W. Janssens, B. A. Poser, A. R. Khan, and K. Uludağ. "Sub-millimeter blood flow mapping of cortical and hippocampal gray matter". In: Proceedings of the 27th Annual Meeting of ISMRM. Montréal, Canada, 2019 




\section{Acknowledgements}

First and foremost, I owe my deepest gratitude to you Kamil. From the very start, you gave me the freedom to explore, learn and purse my interests. I admire your wealth of knowledge across multiple disciplines and I am extremely thankful for the time we spent analysing and learning from the data together. I particularly appreciate your quick replies and regular follow-ups despite all the travel and an extremely packed schedule. I thank you for being an inspirational supervisor and a generous educator. Beyond pulling off these roles with great aplomb, I am especially indebted to you for being a kind human being during the darkest moment of my life, given how early on in my $\mathrm{PhD}$ it was. Thank you immeasurably for your constant support, reassurance and patience. On a lighter note, I am particularly happy about two things during my PhD; first, that you said that I am too data critical and second, that my puns (and jokes) are cringeworthy even by your standards.

Dimo, you truly are, a gentleman and a scholar. I owe everything I learned about sequences, scanning and more, to you and I am extremely grateful for all of that. Thank you for the time and guidance you have given me, through your infinite patience, during both my Master thesis and the PhD. You have been a pillar of support both on and off the academic "pitch" and words do not do justice for how much I appreciate that. You have been a kind and understanding supervisor, teacher, colleague and friend to me. Despite the fact that your time and energy have often been behind the curtains, therefore, I would like to take this opportunity to express my sincerest appreciation for everything you have done. Thank you.

Ben, even though our interaction during the early period of my $\mathrm{PhD}$ was limited, I am very happy that it has since changed. I really appreciate your being available whenever I have questions or doubts that need clarified, thank you for being a great mentor. I am amazed at your ability to juggle a gazillion things, yet manage to find time to fix tiny bugs and make improved sequences for us to work with. It is rather difficult to put into words all the things that I want express my gratitude for, but most of all, I really appreciate your being positive, encouraging of ideas and for your confidence and faith in me. Thanks!

Martin, I remember the first time we discussed layers and I am glad we did. Your attention to the minutiae of data is incredible and I thank you for sharing your expertise with me. Thank you for being your frank and critical self and I have always appreciated your honesty. You do not cease to amaze me with your data visualisation skills. I hope to catch up to you some day. Thank you. 
Renzo, thank you for being an overall upstanding, awesome person. You inspire me through your actions and I really admire the way you conduct yourself on a day-to-day basis. You have been a mentor to me albeit with no formalities. With people clamouring all over the world for a piece of your expertise, you remain an approachable and down-to-earth human being, I am grateful for sharing your knowledge and time with me. Thank you.

Joel, thank you for being a fantastic supervisor during my Masters thesis. That period was invaluable for me and I express my deepest appreciation for your patience and openness to let me try obscure algorithms and tools, and never ceased to encourage me despite hurdles and small hiccoughs that came along the way. Thank you for everything over the years. And yes, I will manage to segment that last hippocampus now that this is finally over.

Peter and Milene, you were the first two people from Maastricht I ever got to know. I am grateful to you for being such an integral part of my education. Thank you for being encouraging and supportive when the going got tough. I really appreciate it.

Rainer, my interest in fMRI became concrete due to your infectious enthusiasm and passion during your lectures and courses. Thank you.

This list would not be complete without thanking the people who gave me the opportunity to switch to basic sciences and allowed me to pursue my interest in neurophysiology. Shankar sir, thank you for having me as a part of your lab. I am grateful for your constant support and encouragement during my traineeship and afterwards. To Christofer, Bhagya and Mahati, you held your patience through my incessant questioning and introduced me to a way of thinking and working that has shaped the rest of my student and research career. Thank you for everything.

Christl and Riny, I am very grateful for your constant support and timely help on innumerous occasions. I really appreciate it, thanks. Eva and José, thank you for all your help and efforts, without which it would be impossible for a newbie to deal with all those forms.

Erik and Linnea, I want to thank you for giving me an opportunity to teach in your laboratory courses at MSP. Not only did it give me valuable teaching experience but also an opportunity to be in a laboratory environment that gave me joy in spite of the arduous travel to Chemelot. And Erik, the puns and jokes we shared were precious and helped shorten those long days. Thanks!

Alard, many thanks for your constructive feedback and advice during my progress reports and our brief conversations. I really appreciate it.

To all the members of the MR-Methods Group, Deni, Gilad, Tobias, Greg, Debbie. Thanks for being such an amazing bunch of people. I am really happy to be a part of the team. 
I express my sincere gratitude to all my colleagues who have played their part in my journey so far. Appreciate each and every thing.

I would be remiss if I did not take this opportunity to thank everyone who has contributed code and/or developed tools, especially the ANTs, CBS-Tools (now Nighres), Freesurfer, FSL, SPM, ITK-SNAP teams. Every line of code, every in-line comment is highly appreciated.

Franc, you are the ultimate bro. Thank you for always being there. Despite the ups and downs, you kindness has been the "steady-state" (sorry, had to sneak one in) for a lot of people, none more so than me. You have been helpful in many ways and I enjoyed all the walks, the board game evenings and generally hanging, be it in Maastricht or abroad. Have a great time in Hamburg, I hope you never stop being yourself.

Arko, my brother, its not often that two people (out of a billion) can see eye-to-eye on so many things and share similar interests. I enjoyed our conversations together and I really appreciate everything you have done. While I hope that you acquire the taste for board-gaming at some point, I really appreciate that you get my nerd/pop-culture references, despite making you cringe, I mean, who else would react if I said, "Wazzup, my glip-glops?".

Robbo, the Spectre of Oxfordlaan, where do I even begin?! Probably Singapore, 2016 and the rest is history (technically 2016 is history too, but I digress). I really enjoyed every single cringeworthy, punfilled, Matrix/LotR-reference riddled conversation we have had over the years and especially the fact how it spirals out of control, much to our shared amusement. Thanks bro.

Sven, my fellow horseman, being the only biologist in the room, I enjoyed all the great, insightful discussions we had. I think talking about stains and biochemical reactions were welcome distractions for me. I really appreciate all the fun we had during every game/movie evening and you have earned my respect for braving spicy food. And despite our disagreement on the sheer, undisputable awesomeness of the Green Lantern, I am happy to call you my friend. Thanks bro.

Roy, buddy, thank you for so many things, including helping me move. I knew I could count on you as a participant for scanning anytime and I could not have pulled off CBS-Tools-ing and Freesufer-ing without your help. I'm glad I could share the anguish of last season's Champions League semi-final defeat of Ajax with you, hopefully, share more joyous instances in the future. Good luck with the hippocampus.

Nikos, my interdepartmental bro, one cannot simply nerd out over non-linear warps or R-markdown over lunch, but we did. Thanks a lot for the fun conversations, I appreciate every single moment. Also, thanks for introducing us to Greek cuisine, it has quickly become one of our favourites. 
Ingo and Marian thanks for sharing this journey with me. While we didn't formally collaborate over anything, we shared so many ups and downs but we brainstormed and resolved them together. I really appreciate it. You guys also gave one of my favourite birthday presents after the Football World Cup 2014. It's been an absolute pleasure getting to know you and I'm happy to call you my friends, scratch that, my bros! And the trinity would not be complete without Faruk, keep blowing those barriers to free and accessible science apart and its always great to hear all the fun, new things you are upto. Thanks bro.

Bhargava, thank you for continuing to play the role of my older sibling, its not been easy. Thanks for sharing your experience, advice and guiding me at crucial moments in my life. You have been my guardian of sorts in Europe and you have been there whenever I needed you. Your presence and support has meant the world to me over the years and although the length of our conversations has reduced, I treasure every moment, thank you for being my bro.

Praveen, ours is the second-generation of a strong friendship, I'm sure our fathers are very proud. It is strange that the moving outside our motherland brought us closer together but I'm happy it did. The time we've spent has been an absolute joy and thank you for everything.

Abhi, nothing I write here can do justice to how much I cherish and value our friendship. Ours is probably one of the longest running, long-distance relationships, isn't it?! Ha ha. I am truly fortunate to have crossed paths with you and your endearing family who have made me one of their own. Thanks man.

Gopi anna, no words can express my gratitude to you for everything you have done for us. You have been a guardian and a pillar of support, which lifted an enormous weight off my shoulders and I am forever indebted to you. My thanks just do not cut it, I know you understand what I mean.

Dodamma and Dodappa, you have been my godparents and it is difficult to express in words the deep sense of gratitude I feel for everything you have done for me. It is not my place to say thanks. I can only say that I am fortunate to have you both in my life and I appreciate everything you have done.

Chaitra, my wife, my best friend and the love of my life, none of this would be possible without your care and support. You have lifted my spirits when I was down, you have inspired me when I was lost, you have stuck by my side always and I cannot find the words to express how much I appreciate everything you do. We are an awesome team.

Amma, you are my role model. I have had the privilege of being raised by such a strong, independent woman. Nothing can measure up to your immense sacrifice, selfless labour, infinite affection and uncon- 
ditional love. You are my shield, my strength and I owe everything I am to you. I am the most fortunate kid in the world, your son.

Appa, you have the final word, as you did so many times. I really wish you were around to read this yourself. Nevertheless, I am glad there were no things left unsaid and I am content knowing that I made you proud.

Sriranga Kashyap

Maastricht

2019-08-29 



\section{Curriculum Vitae}

Śrīranga Kaśhyap ${ }^{1}$ was born on the 8th of May 1989 in Davanagere, Karnataka, India and spent his all of formative years in the beautiful, heritage city of Mysuru, Karnataka, India. In 2007, he pursued a four year Bachelor of Engineering degree in Biotechnology from Visvesvaraya Technological University, Karnataka, India, as it perfectly fit his interest in the technical aspects of biological sciences. He carried out his Bachelor's thesis research in the Department of Neurophysiology at the National Institute of Mental Health and Neuro Sciences (NIMHANS), Bengaluru, India under the supervision of Dr. Christofer Thomas and Prof. B. S. Shankaranarayana Rao studying the effects of chronic stress induced anxiety-like behaviour in rats. Upon graduating with distinction in 2011, he continued his training at NIMHANS on management of laboratory animals, microscopy and histological methods, and psychopharmacological rodent experiments. In 2012, he started the Research Master's programme in Cognitive Neuroscience at Maastricht University. In this period, he acquired time-series data analysis skills during a short internship with Dr. Francesco Gentile and Prof. Bernadette Jansma (Maastricht University) working on automatic artefact detection, denoising and processing of EEG data. For his Master's thesis, he carried out a high-resolution $7 \mathrm{~T}$ MRI study characterising the quantitative MRI properties of the human hippocampus under the supervision of Dr. Joel Reithler and Prof. Peter de Weerd (Maastricht University). In 2014, he started a PhD under the supervision of Prof. Kâmil Uludağ and working closely with Dr. Dimo Ivanov and Dr. Martin Havlicek on laminar fMRI acquisition methods and analyses using $7 \mathrm{~T}$ and 9.4 T MRI at Maastricht University. Following the completion of his PhD, Sriranga continues his research work as a post-doctoral fellow in the MR-Methods group of Dr. Benedikt Poser (Maastricht University) in collaboration with Prof. David Feinberg (University of California, Berkeley, USA).

\footnotetext{
${ }^{1}$ International Alphabet of Sanskrit Transliteration
} 
